June 3, 2020

\title{
BRANCHING RULES AND COMMUTING PROBABILITIES FOR TRIANGULAR AND UNITRIANGULAR MATRICES
}

\author{
DILPREET KAUR, UDAY BHASKAR SHARMA, AND ANUPAM SINGH
}

\begin{abstract}
This paper concerns the enumeration of simultaneous conjugacy classes of $k$-tuples of commuting matrices in the upper triangular group $G T_{n}\left(\mathbf{F}_{q}\right)$ and unitriangular group $U T_{m}\left(\mathbf{F}_{q}\right)$ over the finite field $\mathbf{F}_{q}$ of odd characteristic. This is done for $n=2,3,4$ and $m=3,4,5$, by computing the branching rules. Further, using the branching matrix thus computed, we explicitly get the commuting probabilities $c p_{k}$ for $k \leq 5$ in each case.
\end{abstract}

\section{INTRODUCTION}

Simultaneous conjugacy of commuting $k$-tuples in a group is understood by computing its branching matrix. In [Sh1] and [SS], the branching table/matrix of finite general linear, unitary and symplectic groups of small rank is computed. In this paper, we continue the work for certain solvable groups, namely, upper triangular matrices. Since, this work is continuation of that in [SS], we refer a reader to the same for definition of branching and other related notation. We work with the groups of upper-triangular invertible matrices, $G T_{n}\left(\mathbf{F}_{q}\right)$, and the groups of upper unitriangular matrices $U T_{n}\left(\mathbf{F}_{q}\right)$, over a finite field $\mathbf{F}_{q}$ of odd characteristic. We compute the branching matrix for $G T_{2}\left(\mathbf{F}_{q}\right)$ (Theorem 2.1), $G T_{3}\left(\mathbf{F}_{q}\right)$ (Theorem 3.1), $G T_{4}\left(\mathbf{F}_{q}\right)$ (Theorem4.1), $U T_{3}\left(\mathbf{F}_{q}\right)$ (Theorem 5.1), $U T_{4}\left(\mathbf{F}_{q}\right)$ (Theorem 6.1) and $U T_{5}\left(\mathbf{F}_{q}\right)$ (Theorem [.1).

Further, for a group $G$, the relation of branching matrix $B_{G}$ to commuting probabilities $c p_{k}(G)$ was explored in [SS, Theorem 1.1]. This relation is further explored in the survey article [SS2], where commuting probabilities $c p_{k}(G)$ up to $k \leq 5$ is computed for $G=G L_{2}\left(\mathbf{F}_{q}\right), G L_{3}\left(\mathbf{F}_{q}\right), U_{2}\left(\mathbf{F}_{q}\right)$ and $U_{3}\left(\mathbf{F}_{q}\right)$. It was also proved that $c p_{k}\left(G L_{2}\left(\mathbb{F}_{q}\right)\right)=$ $c p_{k}\left(U_{2}\left(\mathbf{F}_{q}\right)\right)$ for all $k$ even though the branching matrices of the two groups are not same (see Proposition 3.3 [SS2]). In [GR] (see Theorem 12) bounds for commuting probability $c p_{2}$, when $G$ is a solvable group or $p$-group, is computed. Using the branching matrix we compute the commuting probabilities $c p_{k}$, up to $k \leq 5$, for each of the groups $G T_{n}\left(\mathbf{F}_{q}\right)$ and $U T_{n}\left(\mathbf{F}_{q}\right)$ for which we have branching matrix (see Section 8 ).

2010 Mathematics Subject Classification. 05A05,20G40,20E45.

Key words and phrases. Triangular group, Unitriangular group, Commuting tuples of matrices, Branching rules, Commuting probability.

The third named author would like to acknowledge support of SERB grant CRG/2019/000271 during this project. 
For this work, we need conjugacy class types or z-classes (as defined in [SS] and also dealt in $[\mathrm{Bh}]$ ). This is defined as follows: two matrices are said to be of the same conjugacy class type/z-class, if the centralizers of two elements are conjugate. However, a further weaker version is enough for our purpose here. We say that two matrices are of same type if their centralizers are isomorphic. This helps us reduce the size of computation (and size of branching matrix) and causes no loss of generality. Throughout, we assume $q$ is odd. We hope our computation throws some light on the subject of commuting probability and will help us understand the groups better.

Acknowledgments. The authors would like to thank Amritanshu Prasad, IMSc Chennai, for his interest in this work.

\section{BRANCHING RULES FOR $G T_{2}\left(\mathbf{F}_{q}\right)$}

There are four conjugacy class types in $G L_{2}\left(\mathbf{F}_{q}\right)$ given by the following partitions (as in [Sh1] $)(1,1)_{2},(2)_{1},(1)_{1}(1)_{1}$, and $(1)_{2}$. We use this to get the same for $G T_{2}\left(\mathbf{F}_{q}\right)$. Since we are looking at $G T_{2}\left(\mathbf{F}_{q}\right)$, the last one, $(1)_{2}$ doesn't exist in $G T_{2}\left(\mathbf{F}_{q}\right)$. In this paper, we shall not use the partition based nomenclature for the conjugacy class types. Instead we use alphanumeric nomenclature as follows (similar to the pattern in [SS]).

\begin{tabular}{|c|c|c|c|}
\hline Canonical Form & No. of Classes & Centralizer & Name of Class Type \\
\hline $\begin{array}{l}\left(\begin{array}{cc}a & 0 \\
0 & a\end{array}\right) \\
a \in \mathbf{F}_{q}^{*}\end{array}$ & $q-1$ & $G T_{2}\left(\mathbf{F}_{q}\right)$ & $C$ \\
\hline $\begin{array}{l}\left(\begin{array}{ll}a & 1 \\
0 & a\end{array}\right), \\
a \in \mathbf{F}_{q}^{*}\end{array}$ & $q-1$ & $\left\{\left(\begin{array}{cc}x_{0} & x_{1} \\
& x_{0}\end{array}\right) \mid x_{0} \in \mathbf{F}_{q}^{*}\right\}$ & $R_{1}$ \\
\hline $\begin{array}{c}\left(\begin{array}{ll}a & 0 \\
0 & b\end{array}\right), \\
a, b \in \mathbf{F}_{q}^{*}, a \neq b\end{array}$ & $(q-1)(q-2)$ & $\left\{\left({ }^{x_{0}}{ }_{z_{0}}\right) \mid x_{0}, z_{0} \in \mathbf{F}_{q}^{*}\right\}$ & $R_{2}$ \\
\hline
\end{tabular}

Theorem 2.1. The branching rules are summarized in the table below given by the branching matrix:

$$
B_{G T_{2}\left(\mathbf{F}_{q}\right)}=\left(\begin{array}{ccc}
q-1 & 0 & 0 \\
q-1 & q(q-1) & 0 \\
(q-1)(q-2) & 0 & (q-1)^{2}
\end{array}\right) .
$$

We mention the branching rules below.

Proposition 2.2. For an upper triangular matrix of type $C$, the branching rules are as mentioned in the table above.

Proof. The result follows, as this type is central.

Proposition 2.3. For matrices of any of the two regular types:

- A matrix of type $R_{1}$ has $q(q-1)$ branches of type $R_{1}$, and 
- A matrix of type $R_{2}$ has $(q-1)^{2}$ branches of type $R_{2}$.

Proof. The centralizer of a matrix of any of the above mentioned regular types is commutative, hence each element of the centralizer is a branch.

Proof of Theorem 2.1. The branching rules stated in the above propositions, are summarised in the the branching matrix, as mentioned in the statement of the thoerem.

\section{BRAnChing in $G T_{3}(q)$}

Now, we compute the branching table for $G T_{3}\left(\mathbf{F}_{q}\right)$. The table for the conjugacy classes and their types are as follows:

\begin{tabular}{|c|c|c|c|}
\hline Class Representative & Number of Classes & Centralizer size & Name of Type \\
\hline$a I_{3}, a \neq 0$ & $q-1$ & $(q-1)^{3} q^{3}$ & $C$ \\
\hline $\begin{array}{c}\left(\begin{array}{ccc}a & 1 & \\
& a & \\
& & a\end{array}\right),\left(\begin{array}{lll}a & & \\
& a & 1 \\
& & a\end{array}\right), \\
a \neq 0\end{array}$ & $2(q-1)$ & $(q-1)^{2} q^{2}$ & $A_{1}$ \\
\hline$\left(\begin{array}{ccc}a & & 1 \\
& a & \\
& a\end{array}\right), a \neq 0$ & $q-1$ & $(q-1)^{2} q^{3}$ & $A_{2}$ \\
\hline 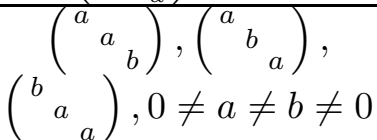 & $3(q-1)(q-2)$ & $(q-1)^{3} q$ & $B_{1}$ \\
\hline$\left(\begin{array}{ccc}a & 1 & \\
& a & 1 \\
& a\end{array}\right), a \neq 0$ & $q-1$ & $(q-1) q^{2}$ & $R_{1}$ \\
\hline 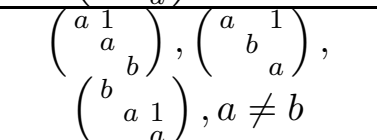 & $3(q-1)(q-2)$ & $(q-1)^{2} q$ & $R_{2}$ \\
\hline 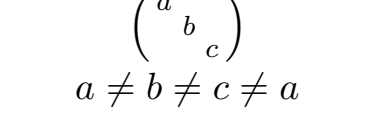 & $(q-1)(q-2)(q-3)$ & $(q-1)^{3}$ & $R_{3}$ \\
\hline
\end{tabular}

The branching rules are described by the branching matrix as follows.

Theorem 3.1. The branching matrix for the group $G T_{3}\left(\mathbf{F}_{q}\right)$ with types written in the order $\left\{C, A_{1}, A_{2}, B_{1}, R_{1}, R_{2}, R_{3}\right\}$ is $B_{G T_{3}\left(\mathbf{F}_{q}\right)}$

$$
=\left(\begin{array}{ccccccc}
q-1 & 0 & 0 & 0 & 0 & 0 & 0 \\
2(q-1) & q(q-1) & 0 & 0 & 0 & 0 & 0 \\
q-1 & 0 & q(q-1) & 0 & 0 & 0 & 0 \\
3(q-1)(q-2) & 0 & 0 & (q-1)^{2} & 0 & 0 & 0 \\
q-1 & q(q-1) & q^{2}-1 & 0 & (q-1) q^{2} & 0 & 0 \\
3(q-1)(q-2) & q(q-1)(q-2) & q(q-1)(q-2) & (q-1)^{2} & 0 & (q-1)^{2} q & 0 \\
(q-1)(q-2)(q-3) & 0 & 0 & (q-1)^{2}(q-2) & 0 & 0 & (q-1)^{3}
\end{array}\right) .
$$

Proposition 3.2. For an upper triangular matrix of type $C$, the branches are as in the second column of the table in the the opening paragraph of this section.

Proof. The result follows, since the matrices of type $C$ are central.

Proposition 3.3. An upper triangular matrix of type $A_{1}$ has $q(q-1)$ branches of type $A_{1}, q(q-1)$ branches of type $R_{1}$, and $q(q-1)(q-2)$ branches of type $R_{2}$. 
Proof. Let $A=\left(\begin{array}{ccc}a & 1 & \\ & a & \\ & & a\end{array}\right)$, a matrix of type $A_{1}$. The centralizer of $A$ is: $Z_{G T_{3}\left(\mathbf{F}_{q}\right)}(A)=$ $\left\{\left(\begin{array}{ccc}x_{0} & x_{1} & x_{2} \\ & x_{0} & \\ & & z_{0}\end{array}\right) \mid x_{0}, z_{0} \neq 0\right\}$. Let $X=\left(\begin{array}{ccc}x_{0} & x_{1} & x_{2} \\ & x_{0} & \\ & & z_{0}\end{array}\right)$, be an invertible member of $Z_{G T_{3}\left(\mathbf{F}_{q}\right)}(A)$. Let $B=\left(\begin{array}{ccc}a_{0} & a_{1} & a_{2} \\ & a_{0} & \\ & & c_{0}\end{array}\right)$, and $B^{\prime}=\left(\begin{array}{ccc}a_{0} & a_{1}^{\prime} & a_{2}^{\prime} \\ & a_{0} & \\ & & c_{0}\end{array}\right)=X B X^{-1}$. Thus equating $X B=B^{\prime} X$ leads us to the following equations:

$$
\begin{aligned}
a_{1}^{\prime} & =a_{1} \\
x_{0} a_{2}+x_{2} c_{0} & =x_{2} a_{0}+z_{0} a_{2}^{\prime}
\end{aligned}
$$

Case: $a_{0}=c_{0}$. Here, equation 3.2 becomes $x_{0} a_{2}=z_{0} a_{2}^{\prime}$. When $a_{2}=0$, then, we have $B$ reduced to $\left(\begin{array}{ccc}a_{0} & a_{1} & \\ & a_{0} & \\ & & a_{0}\end{array}\right)$, with $Z_{G T_{3}\left(\mathbf{F}_{q}\right)}(A, B)=Z_{G T_{3}\left(\mathbf{F}_{q}\right)}(A)$. Thus $(A, B)$ is of type $A_{1}$, and there are $q(q-1)$ such branches.

When $a_{2} \neq 0$, choose $z_{0}$ so that $a_{2}=1$. Then $B$ is reduced to $\left(\begin{array}{ccc}a_{0} & a_{1} & 1 \\ & a_{0} & \\ & & a_{0}\end{array}\right)$, and $Z_{G T_{3}\left(\mathbf{F}_{q}\right)}(A, B)=\left\{\left(\begin{array}{ccc}x_{0} & x_{1} & x_{2} \\ & x_{0} & \\ & & x_{0}\end{array}\right)\right\}$. This subgroup is commutative. Thus $(A, B)$ is of type $R_{1}$, and there are $q(q-1)$ such branches. There are no further cases to see here. Case: $a_{0} \neq c_{0}$. In Equation [3.2, choose $x_{2}$ so that $a_{2}^{\prime}=0$. Thus, $B$ is reduced to $\left(\begin{array}{ccc}a_{0} & a_{1} & \\ & a_{0} & \\ & & c_{0}\end{array}\right)$, and $Z_{G T_{3}\left(\mathbf{F}_{q}\right)}(A, B)=\left\{\left(\begin{array}{ccc}x_{0} & x_{1} & \\ & x_{0} & \\ & & z_{0}\end{array}\right)\right\}$. This subgroup is commutative. Thus $(A, B)$ is of type $R_{2}$, and there are $q^{2}(q-1)=q^{3}-q^{2}$ such branches.

These are all the cases here. Thus, we have a total of $q^{2}+q^{3}-q^{2}=q^{3}$ branches of type $R$.

Proposition 3.4. An upper triangular matrix of type $A_{2}$ has $q(q-1)$ branches of type $A_{2}$, and $q^{2}-1$ branches of type $R_{1}$, and $q(q-1)(q-2)$ branches of type $R_{2}$. 
Proof. Given $A=\left(\begin{array}{ccc}a & & 1 \\ & a & \\ & & a\end{array}\right)$, the canonical form of a matrix of type $A_{2}$. The centralizer of $A, Z_{G T_{3}\left(\mathbf{F}_{q}\right)}(A)$ is $\left\{\left(\begin{array}{ccc}x_{0} & x_{1} & x_{2} \\ & y_{0} & y_{1} \\ & & x_{0}\end{array}\right) \mid x_{0}, y_{0} \neq 0\right\}$. Let $X=\left(\begin{array}{ccc}x_{0} & x_{1} & x_{2} \\ & y_{0} & y_{1} \\ & & x_{0}\end{array}\right) \in$ $Z_{G T_{3}\left(\mathbf{F}_{q}\right)}(A)$. Let $B=\left(\begin{array}{ccc}a_{0} & a_{1} & a_{2} \\ & b_{0} & b_{1} \\ & & a_{0}\end{array}\right)$, and $B^{\prime}=\left(\begin{array}{ccc}a_{0} & a_{1}^{\prime} & a_{2}^{\prime} \\ & b_{0} & b_{1}^{\prime} \\ & & a_{0}\end{array}\right)=X B X^{-1}$. Thus equating $X B=B^{\prime} X$ gives us the following equations:

$$
\begin{aligned}
x_{0} a_{1}+x_{1} b_{0} & =x_{1} a_{0}+y_{0} a_{1}^{\prime} \\
y_{0} b_{1}+y_{1} a_{0} & =x_{0} b_{1}^{\prime}+y_{1} b_{0} \\
x_{0} a_{2}+x_{1} b_{1} & =x_{0} a_{2}^{\prime}+y_{1} a_{1}^{\prime}
\end{aligned}
$$

Using these we reduce $B$ to the mentioned branches.

Proposition 3.5. An upper triangular matrix of type $B_{1}$ has $(q-1)^{2}$ branches of type $B_{1}$, and $(q-1)^{2}$ branches of type $R_{2}$, and $(q-1)^{2}(q-2)$ branches of type $R_{3}$.

Proof. One of the canonical forms of an upper triangular matrix of type $B_{1}$ is $A=$ $\left(\begin{array}{ll}a I_{2} & \\ & b\end{array}\right)$, where $a \neq b \in \mathbf{F}_{q}^{*}$. Hence the centralizer of $A$ is

$$
Z_{G T_{3}\left(\mathbf{F}_{q}\right)}(A)=\left\{\left(\begin{array}{cc}
X & \\
& z_{0}
\end{array}\right) \mid X \in G T_{2}\left(\mathbf{F}_{q}\right), z_{0} \neq 0\right\}
$$

Thus the branches of $A$ are of the form $\left(\begin{array}{ll}C & \\ & d\end{array}\right)$, where $d \neq 0$, and $C$ is a conjugacy class of $G T_{2}\left(\mathbf{F}_{q}\right)$. Hence, the result.

Proposition 3.6. For matrices of the Regular types:

- A matrix of type $R_{1}$ has $(q-1) q^{2}$ branches of type $R_{1}$.

- For type $R_{2}$, there are $(q-1)^{2} q$ branches of type $R_{2}$.

- For type $R_{3}$, there are $(q-1)^{3}$ branches of type $R_{3}$

Proof. The result follows, as the centralizers of matrices of any of the Regular types are commutative.

Proof of Theorem 3.1. From the data in Propositions 3.2 to 3.6, the branching rules are summarized to the branching table/matrix described in the statement of the theorem. 


\section{BRANChing FOR $G T_{4}(q)$}

In this section, we discuss the simultaneous conjugacy classes of tuples of commuting matrices of $G T_{4}\left(\mathbf{F}_{q}\right)$. The conjugacy classes of $G T_{4}\left(\mathbf{F}_{q}\right)$ is described in Appendix A The branching rules are as follows (types written in the order listed in last column of Appendix (A):

Theorem 4.1. The branching matrix for $G T_{4}\left(\mathbf{F}_{q}\right)$ is of size 28 (22 types of $G T_{4}\left(\mathbf{F}_{q}\right)$ and 6 new types), which we write as $B_{G T_{4}\left(\mathbf{F}_{q}\right)}=(\mathcal{A}|\mathcal{B}| \mathcal{C})$ (split in three parts along the columns for convenience of writing) described in Table 1, 2] and 3 .

For the convenience, the branching of non-regular types are in part $\mathcal{A}$, those of regular types in part $\mathcal{B}$, and those of the new types in part $\mathcal{C}$. In each of the sub-tables, the regular branches are in blue, and the new types in red. The $0_{r, s}$ denotes the zero matrix of size $r \times s$. Rest of the section is devoted to proof of this. 
TABle 1 . The matrix $\mathcal{A}$

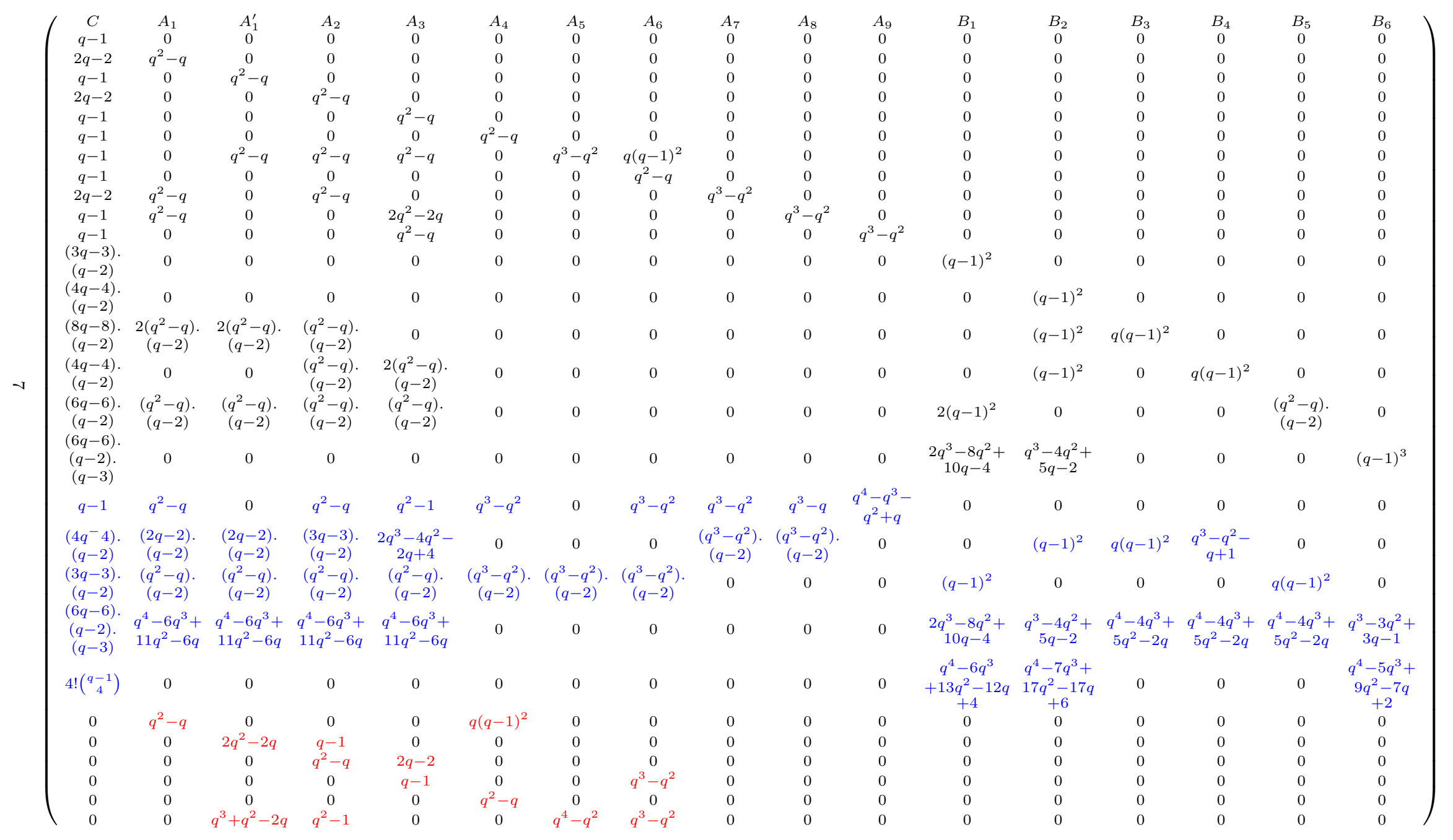


TABLE 2. The matrix $\mathcal{B}$

$$
\mathcal{B}=\left(\begin{array}{ccccc}
R_{1} & R_{2} & R_{3} & R_{4} & R_{5} \\
& & & & \\
& & & & \\
q^{4}-q^{3} & 0 & 0 & 0 & 0 \\
0 & q^{4}-2 q^{3}+q^{2} & 0 & 0 & 0 \\
0 & 0 & q^{4}-2 q^{3}+q^{2} & 0 & 0 \\
0 & 0 & 0 & q^{4}-3 q^{3}+3 q^{2}-q & 0 \\
0 & 0 & 0 & 0 & (q-1)^{4} \\
& & 0 & &
\end{array}\right)
$$

The first column of $A$ corresponds to the central type $C$ and the entries in the column are number of classes of each type in $G T_{4}\left(\mathbf{F}_{q}\right)$ which is the column two of table in Appendix A. For all the regular types $R_{1}, R_{2}, R_{3}, R_{4}$ and $R_{5}$, the only branch is that type itself, and the the number of branches is the size of its centralizer which is again listed in Appendix A. This fully describes the matrix $\mathcal{B}$. Thus, it only remain to explain the matrix $\mathcal{A}$ and $\mathcal{C}$.

4.1. Branching rules for type $A$. Let us deal with type $A$ classes as in Section A.

TABLE 3. The matrix $\mathcal{C}$

$\mathcal{C}=\left(\begin{array}{cccccc}t N T_{1} & t N T_{2} & t N T_{3} & t N T_{4} & t N T_{5} & N R_{1} \\ & \multicolumn{7}{c}{0_{17 \times 6}} & & & \\ & \multicolumn{7}{c}{q^{3}} & 0 & q^{3}-q^{2} & q^{4}-2 q^{3}+q^{2} & q^{4}-q^{3}-q^{2}+q & 0 \\ q^{3}-q^{2} & q^{4}-3 q^{3}+2 q^{2} & q^{4}-3 q^{3}+2 q^{2} & 0 & 0 & 0 \\ 0 & 0 & 0 & 0 & 0 & 0 \\ q^{4}+3 q^{3}+2 q^{2} & 0 & 0 & 0 & 0 & 0 \\ 0 & 0 & 0 & 0 & 0 & 0 \\ 0 & 0 & 0 & 0 & 0 & 0 \\ q^{3}-q^{2} & q^{3}-q^{2} & 0 & 0 & 0 & 0 \\ 0 & 0 & q^{3}-q^{2} & 0 & 0 & 0 \\ 0 & 0 & 0 & q^{3}-q^{2} & 0 & 0 \\ 0 & 0 & 0 & 0 & q^{3}-q & 0 \\ 0 & q^{4}-q^{2} & q^{3}-q & q^{4}-q^{3}-q^{2}+q & 0 & q^{5}-q^{4}\end{array}\right)$


Proposition 4.2. The branching rules of a matrix of type $A_{1}$ are:

\begin{tabular}{c|c||c|c}
\hline Branch & No. Of Branches & Branch & No. of Branches \\
\hline$A_{1}$ & $q(q-1)$ & $R_{1}$ & $(q-1) q$ \\
$A_{7}$ & $q(q-1)$ & $R_{2}$ & $2 q(q-1)(q-2)$ \\
$A_{8}$ & $q(q-1)$ & $R_{3}$ & $q(q-1)(q-2)$ \\
$B_{3}$ & $2 q(q-1)(q-2)$ & $R_{4}$ & $q(q-1)(q-2)(q-3)$ \\
$B_{5}$ & $q(q-1)(q-2)$ & $t N T_{1}$ & $q(q-1)$. \\
\hline
\end{tabular}

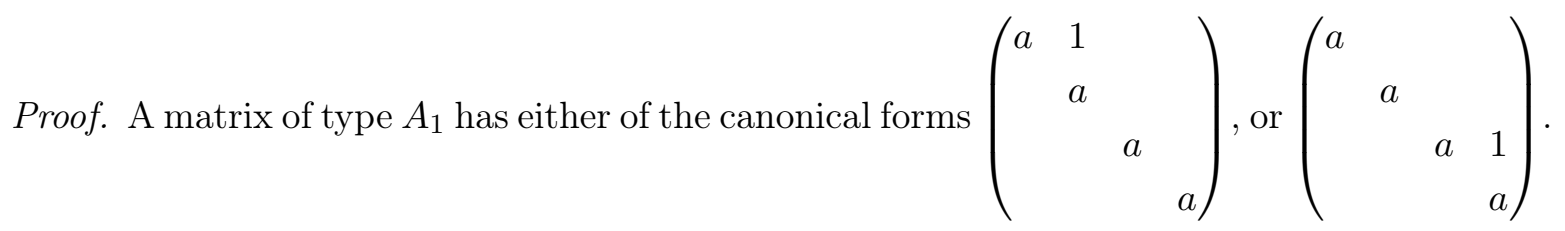

We may consider any one of them. WLOG, we take $A=\left(\begin{array}{cccc}a & 1 & & \\ & a & & \\ & & a & \\ & & & a\end{array}\right)$. The centralizer $Z_{G T_{4}\left(\mathbf{F}_{q}\right)}(A)$ is $\left\{\left(\begin{array}{cccc}a_{0} & a_{1} & a_{2} & a_{3} \\ & a_{0} & & \\ & & c_{0} & c_{1} \\ & & & d_{0}\end{array}\right) \mid a_{0}, c_{0}, d_{0} \neq 0\right\}$. Let $B=\left(\begin{array}{cccc}a_{0} & a_{1} & a_{2} & a_{3} \\ & a_{0} & & \\ & c_{0} & c_{1} \\ & & d_{0}\end{array}\right)$, $B^{\prime}=\left(\begin{array}{cccc}a_{0} & a_{1}^{\prime} & a_{2}^{\prime} & a_{3}^{\prime} \\ & a_{0} & & \\ & & c_{0} & c_{1}^{\prime} \\ & & & d_{0}\end{array}\right) X B X^{-1}$, where $X=\left(\begin{array}{cccc}x_{0} & x_{1} & x_{2} & x_{3} \\ & x_{0} & & \\ & & z_{0} & z_{1} \\ & & & w_{0}\end{array}\right)$. Equating $X B=$

$B^{\prime} X$ leads us to the following:

$$
\begin{aligned}
a_{1}^{\prime} & =a_{1} \\
\left(\begin{array}{cc}
z_{0} & z_{1} \\
& w_{0}
\end{array}\right)\left(\begin{array}{ll}
c_{0} & c_{1} \\
& d_{0}
\end{array}\right) & =\left(\begin{array}{ll}
c_{0} & c_{1}^{\prime} \\
& d_{0}
\end{array}\right)\left(\begin{array}{cc}
z_{0} & z_{1} \\
& w_{0}
\end{array}\right) .
\end{aligned}
$$

Let $C=\left(\begin{array}{cc}c_{0} & c_{1} \\ & d_{0}\end{array}\right)$, and $Z=\left(\begin{array}{cc}z_{0} & z_{1} \\ & w_{0}\end{array}\right)$. The second equation leads us to various conjugacy classes of $G T_{2}\left(\mathbf{F}_{q}\right)$. Hence, we take $C$ to be some conjugacy class representative in $G T_{2}\left(\mathbf{F}_{q}\right)$, and $Z \in Z_{G T_{2}\left(\mathbf{F}_{q}\right)}(C)$. This leads us to the following equation:

$$
x_{0}\left(\begin{array}{ll}
a_{2} & a_{3}
\end{array}\right)+\left(\begin{array}{ll}
x_{2} & x_{3}
\end{array}\right) \cdot\left(C-a_{0} I_{2}\right)=\left(\begin{array}{ll}
a_{2}^{\prime} & a_{3}^{\prime}
\end{array}\right) \cdot Z
$$

When $a_{0}$ is an eigenvalue of $C$ :

When $\left(a_{2}, a_{3}\right)=(0,0)$ : Equation 4.1 becomes $\left(\begin{array}{ll}x_{2} & x_{3}\end{array}\right) \cdot\left(C-a_{0} I_{2}\right)=\left(\begin{array}{ll}0 & 0\end{array}\right)$. 
When $C=a_{0} I_{2}$, Equation 4.1 is void, and we have $B$ reduced to $\left(\begin{array}{cccc}a_{0} & a_{1} & & \\ & a_{0} & & \\ & & a_{0} & \\ & & & a_{0}\end{array}\right)$, and $Z_{G T_{4}\left(\mathbf{F}_{q}\right)}(A, B)=Z_{G T_{4}\left(\mathbf{F}_{q}\right)}(A)$. Thus $(A, B)$ is of type $A_{1}$, and there are $q(q-1)$ such branches.

$$
\text { When } C=\left(\begin{array}{cc}
a_{0} & 1 \\
& a_{0}
\end{array}\right) \text {, Equation } 4.1 \text { becomes }\left(\begin{array}{ll}
x_{2} & x_{3}
\end{array}\right) \cdot\left(\begin{array}{ll}
0 & 1 \\
& 0
\end{array}\right)=\left(\begin{array}{ll}
0 & 0
\end{array}\right) \text {. Thus } x_{2}=0 \text {. }
$$

We have $B$ reduced to $\left(\begin{array}{cccc}a_{0} & a_{1} & & \\ & a_{0} & & \\ & & a_{0} & 1 \\ & & & a_{0}\end{array}\right)$, and $Z_{G T_{4}\left(\mathbf{F}_{q}\right)}(A, B)=\left\{\left(\begin{array}{cccc}x_{0} & x_{1} & & x_{3} \\ & x_{0} & & \\ & & z_{0} & z_{1} \\ & & & z_{0}\end{array}\right)\right\}$.

This centralizer is not isomorphic to the centralizers of te known types. Thus $(A, B)$ is of a new type, which we will call $t N T_{1}$ and there are $q(q-1)$ such branches.

When $C=\left(\begin{array}{ll}a_{0} & \\ & b_{0}\end{array}\right)\left(a_{0} \neq b_{0}\right)$, Equation 4.1 becomes $\left(\begin{array}{ll}x_{2} & x_{3}\end{array}\right) \cdot\left(\begin{array}{l}\left(b_{0}-a_{0}\right)\end{array}\right)=$ $\left(\begin{array}{ll}0 & 0\end{array}\right)$. Thus $x_{3}=0$. We have $B$ reduced to $\left(\begin{array}{cccc}a_{0} & a_{1} & \\ & a_{0} & \\ & & & \\ & & a_{0} & \\ & & b_{0}\end{array}\right)$, and $Z_{G T_{4}\left(\mathbf{F}_{q}\right)}(A, B)=$ $\left\{\left(\begin{array}{cccc}x_{0} & x_{1} & x_{2} & \\ & x_{0} & & \\ & & z_{0} & \\ & & & z_{2}\end{array}\right)\right\}$. Thus $(A, B)$ is of type $B_{3}$, and there are $q(q-1)(q-2)$ such branches.

When $C=\left(\begin{array}{cc}b_{0} & \\ & a_{0}\end{array}\right)\left(a_{0} \neq b_{0}\right)$, Equation 4.1 becomes $\left(\begin{array}{ll}x_{2} & x_{3}\end{array}\right) \cdot\left(\begin{array}{c}\left(b_{0}-a_{0}\right) \\ \end{array}\right)=$ $\left(\begin{array}{ll}0 & 0\end{array}\right)$. Thus $x_{2}=0$. We have $B$ reduced to $\left(\begin{array}{cccc}a_{0} & a_{1} & \\ & a_{0} & \\ & & b_{0} & \\ & & & a_{0}\end{array}\right)$, and $Z_{G T_{4}\left(\mathbf{F}_{q}\right)}(A, B)=$ $\left\{\left(\begin{array}{cccc}x_{0} & x_{1} & & x_{3} \\ & x_{0} & & \\ & & z_{0} & \\ & & & z_{2}\end{array}\right)\right\}$. Thus $(A, B)$ is of type $B_{3}$, and there are $q(q-1)(q-2)$ such branches.

When $\left(a_{2}, a_{3}\right) \neq(0,0)$ :

When $C=a_{0} I_{2}$, Equation 4.1 becomes: $\left(\begin{array}{ll}a_{2} & a_{3}\end{array}\right)=\left(\begin{array}{ll}a_{2}^{\prime} & a_{3}^{\prime}\end{array}\right)\left(\begin{array}{ll}\frac{z_{0}}{x_{0}} & \frac{z_{1}}{x_{0}} \\ & \frac{z_{2}}{x_{0}}\end{array}\right)$. 
We have from this:

$$
\begin{aligned}
& a_{2}=\frac{z_{0}}{x_{0}} a_{2}^{\prime} \\
& a_{3}=\frac{z_{1}}{z_{0}} a_{2}^{\prime}+\frac{z_{2}}{x_{0}} a_{3}^{\prime}
\end{aligned}
$$

When $a_{2} \neq 0$, choose $x_{0}$ such that $a_{2}^{\prime}=1$. In the equation below, choose $z_{1}$ so that $a_{3}^{\prime}=0$.

So, $B$ reduces to $\left(\begin{array}{cccc}a_{0} & a_{1} & 1 & \\ & a_{0} & & \\ & & a_{0} & \\ & & & a_{0}\end{array}\right)$, and $Z_{G T_{4}\left(\mathbf{F}_{q}\right)}(A, B)=\left\{\left(\begin{array}{cccc}x_{0} & x_{1} & x_{2} & x_{3} \\ & x_{0} & & \\ & & x_{0} & \\ & & & z_{2}\end{array}\right)\right\}$.

This $(A, B)$ is of type $A_{7}$, and there are $q(q-1)$ such branches.

When $a_{2}=0, a_{3} \neq 0$, choose $z_{2}$ such that $a_{3}^{\prime}=1$. Thus $B$ is reduced to $\left(\begin{array}{cccc}a_{0} & a_{1} & & 1 \\ & a_{0} & & \\ & a_{0} & \\ & & a_{0}\end{array}\right)$, and $Z_{G T_{4}\left(\mathbf{F}_{q}\right)}(A, B)=\left\{\left(\begin{array}{cccc}x_{0} & x_{1} & x_{2} & x_{3} \\ & x_{0} & & \\ & & z_{0} & z_{1} \\ & & & x_{0}\end{array}\right)\right\}$. This $(A, B)$ is of type $A_{8}$, and there are $q(q-1)$ such branches.

$$
\begin{aligned}
\text { When } C=\left(\begin{array}{cc}
a_{0} & 1 \\
& a_{0}
\end{array}\right) \text {, here } Z=\left(\begin{array}{ll}
x_{0} & x_{1} \\
& x_{0}
\end{array}\right) \text {. Equation 4.1 becomes } \\
\qquad\left(\begin{array}{ll}
a_{2} & a_{3}
\end{array}\right)+\left(\begin{array}{ll}
\frac{x_{2}}{x_{0}} & \frac{x_{3}}{x_{0}}
\end{array}\right)\left(\begin{array}{ll}
0 & 1 \\
0 & 0
\end{array}\right)=\left(\begin{array}{ll}
a_{2}^{\prime} & a_{3}^{\prime}
\end{array}\right)\left(\begin{array}{cc}
\frac{z_{0}}{x_{0}} & \frac{z_{1}}{x_{0}} \\
& \frac{z_{0}}{x_{0}}
\end{array}\right) .
\end{aligned}
$$

We have:

$$
\begin{aligned}
a_{2} & =a_{2}^{\prime} \frac{z_{0}}{x_{0}} \\
a_{3}+\frac{x_{2}}{x_{0}} & =\frac{z_{1}}{z_{0}} a_{2}^{\prime}+\frac{z_{0}}{x_{0}} a_{3}^{\prime} .
\end{aligned}
$$

Choose $x_{2}$ such that $a_{3}^{\prime}=0$. As $\left(a_{2}, a_{3}\right) \neq(0,0)$, and $a_{3}=0$, we have $a_{2} \neq 0$. Choose $z_{0}$ such that $a_{2}^{\prime}=1$. So, $B$ is reduced to $\left(\begin{array}{cccc}a_{0} & a_{1} & 1 & \\ & a_{0} & & \\ & a_{0} & 1 \\ & & a_{0}\end{array}\right)$, and $Z_{G T_{4}\left(\mathbf{F}_{q}\right)}(A, B)=$ $\left\{\left(\begin{array}{cccc}x_{0} & x_{1} & x_{2} & x_{3} \\ & x_{0} & & \\ & & x_{0} & x_{2} \\ & & & x_{0}\end{array}\right)\right\}$. This $(A, B)$ is of type $R_{1}$, and there are $q(q-1)$ such branches. 
When $C=\left(\begin{array}{cc}a_{0} & \\ & b_{0}\end{array}\right)$, where $a_{0} \neq b_{0}$. Here $Z=\left(\begin{array}{ll}z_{0} & \\ & z_{2}\end{array}\right)$. Here, Equation 4.1 becomes:

$$
\left(\begin{array}{ll}
\frac{x_{2}}{x_{0}} & \frac{x_{3}}{x_{0}}
\end{array}\right)\left(\begin{array}{cc}
0 & \\
0 & b_{0}-a_{0}
\end{array}\right)=\left(\begin{array}{ll}
a_{2}^{\prime} & a_{3}^{\prime}
\end{array}\right)\left(\begin{array}{cc}
\frac{z_{0}}{x_{0}} & \\
& \frac{z_{2}}{x_{0}}
\end{array}\right) .
$$

We have:

$$
\begin{aligned}
a_{2} & =a_{2}^{\prime} \frac{z_{0}}{x_{0}} \\
a_{3}+\frac{x_{3}}{x_{0}}\left(b_{0}-a_{0}\right) & =\frac{z_{0}}{x_{0}} a_{3}^{\prime} .
\end{aligned}
$$

As $b_{0}-a_{0} \neq 0$, choose $x_{3}$ so that $a_{3}^{\prime}=0$. So we are left with $a_{2} \neq 0$. Choose $z_{0}$ such that $a_{2}^{\prime}=1$. So $B$ is reduced to $\left(\begin{array}{cccc}a_{0} & a_{1} & 1 & \\ & a_{0} & & \\ & & a_{0} & \\ & & b_{0}\end{array}\right)$, and $Z_{G T_{4}\left(\mathbf{F}_{q}\right)}(A, B)=$ $\left\{\left(\begin{array}{cccc}x_{0} & x_{1} & x_{2} & \\ & x_{0} & & \\ & & x_{0} & \\ & & & z_{2}\end{array}\right)\right\}$. Thus, $(A, B)$ is of type $R_{2}$, and there are $q(q-1)(q-2)$ such branches.

When $C=\left(\begin{array}{cc}b_{0} & \\ & a_{0}\end{array}\right)$, where $a_{0} \neq b_{0}$. Here $Z=\left(\begin{array}{cc}z_{0} & \\ & z_{2}\end{array}\right)$. Here, Equation 4.1 becomes:

$$
\left(\begin{array}{ll}
\frac{x_{2}}{x_{0}} & \frac{x_{3}}{x_{0}}
\end{array}\right)\left(\begin{array}{cc}
b_{0}-a_{0} & \\
& 0
\end{array}\right)=\left(\begin{array}{ll}
a_{2}^{\prime} & a_{3}^{\prime}
\end{array}\right)\left(\begin{array}{ll}
\frac{z_{0}}{x_{0}} & \\
& \frac{z_{2}}{x_{0}}
\end{array}\right) .
$$

We have:

$$
\begin{aligned}
a_{2}+\frac{x_{2}}{x_{0}}\left(b_{0}-a_{0}\right) & =a_{2}^{\prime} \frac{z_{0}}{x_{0}} \\
a_{3} & =\frac{z_{0}}{x_{0}} a_{3}^{\prime} .
\end{aligned}
$$

As $b_{0}-a_{0} \neq 0$, choose $x_{2}$ so that $a_{2}^{\prime}=0$. So we are left with $a_{3} \neq 0$. Choose $z_{2}$ such that $a_{3}^{\prime}=1$. So $B$ is reduced to $\left(\begin{array}{cccc}a_{0} & a_{1} & & 1 \\ & a_{0} & & \\ & & b_{0} & \\ & & a_{0}\end{array}\right)$, and $Z_{G T_{4}\left(\mathbf{F}_{q}\right)}(A, B)=$ $\left\{\left(\begin{array}{cccc}x_{0} & x_{1} & & x_{3} \\ & x_{0} & & \\ & & z_{0} & \\ & & & x_{0}\end{array}\right)\right\}$. Thus, $(A, B)$ is of type $R_{2}$, and there are $q(q-1)(q-2)$ such branches. 
Now, we come to the case of $a_{0}$ not being an eigenvalue of $C$. In Equation 4.1, the matrix $\left(C-a_{0} I_{2}\right)$ is invertible. So, we can choose $x_{2}, x_{3}$ such that both $a_{2}=a_{3}=0$. Thus, on replacing $a_{2}$ and $a_{3}$ with 0 each in Equation 4.1, we get $\left(\begin{array}{ll}x_{2} & x_{3}\end{array}\right)\left(\begin{array}{ll}C-a_{0} I_{2}\end{array}\right)=\left(\begin{array}{ll}0 & 0\end{array}\right)$. So $x_{2}=x_{3}=0$. Thus, we have:

When $C=b_{0} I_{2}, b_{0} \neq a_{0}, B$ is reduced to $\left(\begin{array}{cccc}a_{0} & a_{1} & \\ & a_{0} & & \\ & & b_{0} & \\ & & & b_{0}\end{array}\right)$, and $Z_{G T_{4}\left(\mathbf{F}_{q}\right)}(A, B)=$ $\left\{\left(\begin{array}{cccc}x_{0} & x_{1} & \\ & x_{0} & \\ & & z_{0} & z_{1} \\ & & & z_{2}\end{array}\right)\right\} .(A, B)$ is of the type $B_{5}$, and there are $q(q-1)(q-2)$ such branches.

When $C=\left(\begin{array}{cc}b_{0} & 1 \\ & b_{0}\end{array}\right), b_{0} \neq a_{0}, B$ is reduced to $\left(\begin{array}{cccc}a_{0} & a_{1} & & \\ & a_{0} & & \\ & & b_{0} & 1 \\ & & & b_{0}\end{array}\right)$, and $Z_{G T_{4}\left(\mathbf{F}_{q}\right)}(A, B)=$ $\left\{\left(\begin{array}{cccc}x_{0} & x_{1} & \\ & x_{0} & \\ & & z_{0} & z_{1} \\ & & & z_{0}\end{array}\right)\right\} .(A, B)$ is of the type $R_{3}$, and there are $q(q-1)(q-2)$ such branches.

When $C=\left(\begin{array}{cc}b_{0} & \\ & c_{0}\end{array}\right), b_{0} \neq a_{0} \neq c_{0} \neq b_{0}, B$ is reduced to $\left(\begin{array}{cccc}a_{0} & a_{1} & & \\ & a_{0} & & \\ & & b_{0} & \\ & & c_{0}\end{array}\right)$, and $Z_{G T_{4}\left(\mathbf{F}_{q}\right)}(A, B)=\left\{\left(\begin{array}{cccc}x_{0} & x_{1} & & \\ & x_{0} & & \\ & & z_{0} & \\ & & & z_{2}\end{array}\right)\right\} .(A, B)$ is of the type $R_{4}$, and there are $q(q-$ 1) $(q-2)(q-3)$ such branches.

We are left with no other cases.

Proposition 4.3. The branching rules of a matrix of type $A_{1}^{\prime}$ are: 


\begin{tabular}{c|c||c|c}
\hline Branch & No. of Branches & Branch & No. of Branches \\
\hline$A_{1}^{\prime}$ & $q(q-1)$ & $R_{2}$ & $2 q(q-1)(q-2)$ \\
$A_{5}$ & $q(q-1)$ & $R_{3}$ & $q(q-1)(q-2)$ \\
$B_{3}$ & $2 q(q-1)(q-2)$ & $R_{4}$ & $q(q-1)(q-2)(q-3)$ \\
$B_{5}$ & $q(q-1)(q-2)$ & $N R_{1}$ & $q(q-1)(q+2)$ \\
$t N T_{2}$ & $2 q(q-1)$ & & \\
\hline
\end{tabular}

Two new types $N R_{1}$ and $t N T_{2}$ appear here. The centralizers of these new types are

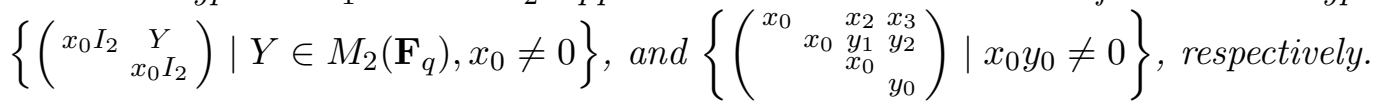

Proof. A matrix of type $A_{1}^{\prime}$ has the canonical form: $A=\left(\begin{array}{llll}a & & & \\ & a & 1 & \\ & a & \\ & & a\end{array}\right)$. The centralizer $Z_{G T_{4}\left(\mathbf{F}_{q}\right)}(A)$, of $A$ is $\left\{\left(\begin{array}{llll}a_{0} & & a_{2} & a_{3} \\ & b_{0} & b_{1} & b_{2} \\ & & b_{0} & \\ & & & c_{0}\end{array}\right)\right\}$. Let $B=\left(\begin{array}{llll}a_{0} & & a_{2} & a_{3} \\ & b_{0} & b_{1} & b_{2} \\ & b_{0} & \\ & & c_{0}\end{array}\right)$, $B^{\prime}=\left(\begin{array}{cccc}a_{0} & & a_{2}^{\prime} & a_{3}^{\prime} \\ & b_{0} & b_{1}^{\prime} & b_{2}^{\prime} \\ & & b_{0} & \\ & & & c_{0}\end{array}\right)=X B X^{-1}$, and where $X=\left(\begin{array}{cccc}x_{0} & & x_{2} & x_{3} \\ & y_{0} & y_{1} & y_{2} \\ & y_{0} & \\ & & z_{0}\end{array}\right)$. Denote the submatrix $\left(\begin{array}{cc}a_{0} & a_{3} \\ & c_{0}\end{array}\right)$ of $B$ by $C$, and the submatrix $\left(\begin{array}{cc}x_{0} & x_{3} \\ & z_{0}\end{array}\right)$ by $Z$. Then equating $X B=B^{\prime} X$ leads us to $Z C=C^{\prime} Z$. Thus, we can take $C$ to be a canonical form in $G T_{2}\left(\mathbf{F}_{q}\right)$, and $Z \in Z_{G T_{2}\left(\mathbf{F}_{q}\right)}(C)$. Thus we have $b_{1}^{\prime}=b_{1}$, and the following equaitons:

$$
\begin{aligned}
x_{0} a_{2}+x_{2} b_{0} & =x_{2} a_{0}+y_{0} a_{2}^{\prime} \\
y_{0} b_{2}+y_{2} c_{0} & =y_{2} b_{0}+z_{0} b_{2}^{\prime}
\end{aligned}
$$

\section{When $C$ has $b_{0}$ as an eigenvalue:}

When $\left(a_{2}, b_{2}\right)=(0,0)$ :

When $C=b_{0} I_{2}$, Equation 4.4 and 4.5 become void, and $B$ becomes, $B=\left(\begin{array}{llll}b_{0} & & & \\ & b_{0} & b_{1} & \\ & & b_{0} & \\ & & & b_{0}\end{array}\right)$, and $Z_{G T_{4}\left(\mathbf{F}_{q}\right)}(A, B)=Z_{G T_{4}\left(\mathbf{F}_{q}\right)}(A) . \quad(A, B)$ is of type $A_{1}^{\prime}$, and there are $q(q-1)$ such branches. 
When $C=\left(\begin{array}{cc}b_{0} & 1 \\ & b_{0}\end{array}\right)$. Here too, Equations 4.4 and 4.5 are void. So, $B$ reduces to $\left(\begin{array}{cccc}b_{0} & & & 1 \\ & b_{0} & b_{1} & \\ & & b_{0} & \\ & & & b_{0}\end{array}\right)$, and $Z_{G T_{4}\left(\mathbf{F}_{q}\right)}(A, B)=\left\{\left(\begin{array}{cccc}x_{0} & & x_{2} & x_{3} \\ & y_{0} & y_{1} & y_{2} \\ & & y_{0} & \\ & & & x_{0}\end{array}\right)\right\}$. This $(A, B)$ is of type $A_{5}$, and there are $q(q-1)$ such branches.

When $C=\left(\begin{array}{cc}b_{0} & \\ & c_{0}\end{array}\right), b_{0} \neq c_{0}$. Here Equation 4.4 stays void, but 4.5 becomes $y_{2} c_{0}=y_{2} b_{0}$, thus $y_{2}=0$. So, $B$ reduces to $\left(\begin{array}{llll}b_{0} & & & \\ & b_{0} & b_{1} & \\ & & b_{0} & \\ & & c_{0}\end{array}\right)$, and $Z_{G T_{4}\left(\mathbf{F}_{q}\right)}(A, B)=$ $\left\{\left(\begin{array}{llll}x_{0} & & x_{2} & \\ & y_{0} & y_{1} & \\ & & y_{0} & \\ & & & z_{0}\end{array}\right)\right\} .(A, B)$ is of type $B_{3}$, and there are $q(q-1)(q-2)$ such branches. When $C=\left(\begin{array}{ll}a_{0} & \\ & b_{0}\end{array}\right), b_{0} \neq a_{0}$. Here Equation 4.4 becomes $x_{2} b_{0}=x_{2} a_{0}$, hence $x_{2}=0$, and Equation 4.5 stays void. So, $B$ reduces to $\left(\begin{array}{llll}c_{0} & & & \\ & b_{0} & b_{1} & \\ & & b_{0} & \\ & & & b_{0}\end{array}\right)$, and $Z_{G T_{4}\left(\mathbf{F}_{q}\right)}(A, B)=$ $\left\{\left(\begin{array}{llll}x_{0} & & & \\ & y_{0} & y_{1} & y_{2} \\ & & y_{0} & \\ & & & z_{0}\end{array}\right)\right\} .(A, B)$ is of type $B_{3}$, and there are $q^{2}(q-1)=q^{3}-q^{2}$ such branches.

When $\left(a_{2}, b_{2}\right) \neq(0,0)$ :

When $C=b_{0} I_{2}$, Equations 4.4 and 4.5 become

$$
\begin{aligned}
x_{0} a_{2} & =y_{0} a_{2}^{\prime} \\
y_{0} b_{2} & =z_{0} b_{2}^{\prime}
\end{aligned}
$$

When $a_{2} \neq 0$, choose $y_{0}$ such that $a_{2}^{\prime}=1$. When $b_{2} \neq 0$, choose $z_{0}$ such that $b_{2}^{\prime}=1$. So, $B$ is reduced to $\left(\begin{array}{cccc}b_{0} & & 1 & \\ & b_{0} & b_{1} & 1 \\ & & b_{0} & \\ & & & b_{0}\end{array}\right)$, and $Z_{G T_{4}\left(\mathbf{F}_{q}\right)}(A, B)=\left\{\left(\begin{array}{llll}y_{0} & & x_{2} & x_{3} \\ & y_{0} & y_{1} & y_{2} \\ & y_{0} & \\ & & & y_{0}\end{array}\right)\right\}$. 
This centralizer is not of any known type in $G T_{4}\left(\mathbf{F}_{q}\right)$, and it is clearly a commutative one. We call this new type $N R_{1}$. There are $q(q-1)$ such branches.

When $b_{2}=0$, then Equation 4.5 is void, and $B$ is reduced to $\left(\begin{array}{cccc}b_{0} & & 1 & \\ & b_{0} & b_{1} & \\ & b_{0} & \\ & & b_{0}\end{array}\right)$, and $Z_{G T_{4}\left(\mathbf{F}_{q}\right)}(A, B)=\left\{\left(\begin{array}{cccc}y_{0} & & x_{2} & x_{3} \\ & y_{0} & y_{1} & y_{2} \\ & & y_{0} & \\ & & & z_{0}\end{array}\right)\right\}$. This centralizer too is not of any known type in $G T_{4}\left(\mathbf{F}_{q}\right)$, and definitely not of $R_{1}$, as this one is 6-dimensional. We call this new type $t N T_{2}$. There are $q^{2}$ such branches.

When $a_{2}=0$, and $b_{2} \neq 0$, choose $z_{0}$ such that $b_{2}^{\prime}=1$, and $B$ is reduced to $\left(\begin{array}{cccc}b_{0} & & & \\ & b_{0} & b_{1} & 1 \\ & & b_{0} & \\ & & & b_{0}\end{array}\right)$, and $Z_{G T_{4}\left(\mathbf{F}_{q}\right)}(A, B)=\left\{\left(\begin{array}{cccc}x_{0} & & x_{2} & x_{3} \\ & y_{0} & y_{1} & y_{2} \\ & & y_{0} & \\ & & & y_{0}\end{array}\right)\right\}$. We have another $q^{2}$ branches of this new type $t N T_{2}$.

When $C=\left(\begin{array}{cc}b_{0} & 1 \\ & b_{0}\end{array}\right)$. Here $Z=\left(\begin{array}{cc}x_{0} & x_{3} \\ & x_{0}\end{array}\right)$. Equation 4.4 becomes $x_{0} a_{2}=y_{0} a_{2}^{\prime}$, and Equation 4.5 becomes $y_{0} b_{2}=x_{0} b_{2}^{\prime}$.

When $a_{2} \neq 0$, choose $y_{0}$ such that $a_{2}^{\prime}=1$. Now, on substituting $a_{2}$ by $a_{2}^{\prime}=1$ in the equation, we get $y_{0}=x_{0}$, and thus $b_{2}^{\prime}=b_{2}$. $B$ is reduced to $\left(\begin{array}{cccc}b_{0} & & 1 & 1 \\ & b_{0} & b_{1} & b_{2} \\ & b_{0} & \\ & & b_{0}\end{array}\right)$, with $Z_{G T_{4}\left(\mathbf{F}_{q}\right)}(A, B)=\left\{\left(\begin{array}{cccc}y_{0} & & x_{2} & x_{3} \\ & y_{0} & y_{1} & y_{2} \\ & & y_{0} & \\ & & & y_{0}\end{array}\right)\right\} .(A, B)$ is of type $N R_{1}$, and there are $q^{2}(q-1)$ such branches.

When $a_{2}=0$, we look at $b_{2}^{\prime} \neq 0$, Choose $x_{0}$ such that $b_{2}^{\prime}=1$. Then $B$ is reduced to $\left(\begin{array}{cccc}b_{0} & & & 1 \\ & b_{0} & b_{1} & 1 \\ & & b_{0} & \\ & & & b_{0}\end{array}\right)$, with $Z_{G T_{4}\left(\mathbf{F}_{q}\right)}(A, B)=\left\{\left(\begin{array}{cccc}y_{0} & & x_{2} & x_{3} \\ & y_{0} & y_{1} & y_{2} \\ & & y_{0} & \\ & & & y_{0}\end{array}\right)\right\} .(A, B)$ is of type $R_{1}$, and there are another $q(q-1)$ such branches. 
When $C=\left(\begin{array}{cc}b_{0} & \\ & c_{0}\end{array}\right), b_{0} \neq c_{0}$, then $Z=\left(\begin{array}{ll}x_{0} & \\ & z_{0}\end{array}\right)$. Equation 4.4 becomes $x_{0} a_{2}=$ $y_{0} a_{2}^{\prime}$, and Equation 4.5 becomes $y_{0} b_{2}+y_{2} c_{0}=y_{2} b_{0}+z_{0} b_{2}^{\prime}$. As $b_{0} \neq c_{0}$, choose $y_{2}$ such that $b_{2}^{\prime}=0$. So we have only one case here $a_{2} \neq 0$. Thus, choose $x_{0}$ such that $a_{2}^{\prime}=1$. Thus $B$ is reduced to $\left(\begin{array}{cccc}b_{0} & & 1 & \\ & b_{0} & b_{1} & \\ & & b_{0} & \\ & & & c_{0}\end{array}\right)$, and $Z_{G T_{4}\left(\mathbf{F}_{q}\right)}(A, B)=\left\{\left(\begin{array}{llll}y_{0} & & x_{2} & \\ & y_{0} & y_{1} & \\ & & y_{0} & \\ & & & z_{0}\end{array}\right)\right\}$. $(A, B)$ is of type $R_{2}$, and there are $q(q-1)(q-2)$ such branches.

When $C=\left(\begin{array}{cc}a_{0} & \\ & b_{0}\end{array}\right), a_{0} \neq b_{0}$, then $Z=\left(\begin{array}{cc}x_{0} & \\ & z_{0}\end{array}\right)$. Equation 4.4 becomes $x_{0} a_{2}+$ $x_{2} b_{0}=y_{0} a_{2}^{\prime}+x_{2} a_{0}$, and Equation 4.5 becomes $y_{0} b_{2}=z_{0} b_{2}^{\prime}$. As $b_{0} \neq c_{0}$, choose $x_{2}$ such that $a_{2}^{\prime}=0$. So we have only one case here $b_{2} \neq 0$. Thus, choose $z_{0}$ such that $b_{2}^{\prime}=1$. Thus $B$ is reduced to $\left(\begin{array}{cccc}a_{0} & & & \\ & b_{0} & b_{1} & 1 \\ & & b_{0} & \\ & & & b_{0}\end{array}\right)$, and $Z_{G T_{4}\left(\mathbf{F}_{q}\right)}(A, B)=\left\{\left(\begin{array}{llll}x_{0} & & & \\ & y_{0} & y_{1} & y_{2} \\ & & y_{0} & \\ & & & y_{0}\end{array}\right)\right\}$. $(A, B)$ is of type $R_{2}$, and there are $q(q-1)(q-2)$ such branches.

Now, the second main case of $b_{0}$ not being an eigenvalue of $C$, i.e., $b_{0} \neq a_{0}$ and $b_{0} \neq c_{0}$. Here in Equation 4.4, choose $x_{2}$ so that $a_{2}^{\prime}=0$, and in Equation 4.5 choose $y_{2}$ so that $b_{2}^{\prime}=0$.

$$
\begin{aligned}
& \text { When } C=a_{0} I_{2} \text {, where } a_{0} \neq b_{0}, B \text { is reduced to }\left(\begin{array}{llll}
a_{0} & & & \\
& b_{0} & b_{1} & \\
& & b_{0} & \\
& & a_{0}
\end{array}\right) \text {, and } Z_{G T_{4}\left(\mathbf{F}_{q}\right)}(A, B)= \\
& \left\{\left(\begin{array}{llll}
x_{0} & & & x_{3} \\
& y_{0} & y_{1} & \\
& & y_{0} & \\
& & & z_{0}
\end{array}\right)\right\} .(A, B) \text { is of type } B_{5} \text {, and there are } q(q-1)(q-2) \text { such branches. } \\
& \text { When } C=\left(\begin{array}{cc}
a_{0} & 1 \\
& a_{0}
\end{array}\right) \text {, where } a_{0} \neq b_{0}, B \text { is reduced to }\left(\begin{array}{cccc}
a_{0} & & & 1 \\
& b_{0} & b_{1} & \\
& & b_{0} & \\
& & a_{0}
\end{array}\right) \text {, and } \\
& Z_{G T_{4}\left(\mathbf{F}_{q}\right)}(A, B)=\left\{\left(\begin{array}{cccc}
x_{0} & & & x_{3} \\
& y_{0} & y_{1} & \\
& & y_{0} & \\
& & & x_{0}
\end{array}\right)\right\} .(A, B) \text { is of type } R_{3} \text {, and there are } q(q- \\
& \text { 1) }(q-2) \text { such branches. }
\end{aligned}
$$


When $C=\left(\begin{array}{cc}a_{0} & \\ & c_{0}\end{array}\right)$, where $a_{0}, c_{0} \neq b_{0}$, and $a_{0} \neq c_{0}, B$ is reduced to $\left(\begin{array}{cccc}a_{0} & & & \\ & b_{0} & b_{1} & \\ & & b_{0} & \\ & & c_{0}\end{array}\right)$, and $Z_{G T_{4}\left(\mathbf{F}_{q}\right)}(A, B)=\left\{\left(\begin{array}{llll}x_{0} & & & \\ & y_{0} & y_{1} & \\ & & y_{0} & \\ & & & z_{0}\end{array}\right)\right\} .(A, B)$ is of type $R$, and there are $q(q-$ 1) $(q-2)(q-3)$ such branches.

With this there are no other cases left for us to analyse.

Adding up the branches of type $N R_{1}$, we have a total of $2 q(q-1)+q^{2}(q-1)=$ $q(q-1)(q+2)$ branches.

Proposition 4.4. The branching rules of a matrix of type $A_{2}$ are given below:

\begin{tabular}{c|c||c|c}
\hline Branch & No. of Branches & Branch & No. of Branches \\
\hline$A_{2}$ & $q(q-1)$ & $R_{2}$ & $3\left(q^{2}-q\right)(q-2)$ \\
$A_{5}$ & $q(q-1)$ & $R_{3}$ & $\left(q^{2}-q\right)(q-2)$ \\
$A_{7}$ & $q(q-1)$ & $R_{4}$ & $q(q-1)(q-2)(q-3)$ \\
$B_{3}$ & $\left(q^{2}-q\right)(q-2)$ & $t N T_{2}$ & $q-1$ \\
$B_{4}$ & $\left(q^{2}-q\right)(q-2)$ & $t N T_{3}$ & $q(q-1)$ \\
$B_{5}$ & $\left(q^{2}-q\right)(q-2)$ & $N R_{1}$ & $q^{2}-1$. \\
$R_{1}$ & $q(q-1)$ & & \\
\hline
\end{tabular}

A further new type $t N T_{3}$ appears here, whose centralizer is $\left\{\left(\begin{array}{cccc}x_{0} & x_{1} & x_{2} & x_{3} \\ & y_{0} & y_{1} & y_{2} \\ & x_{0} & \\ & & x_{0}\end{array}\right) \mid x_{0}, y_{0} \neq 0\right\}$.

Proof. Matrices of this type have two non-similar canonical forms: $\left(\begin{array}{cccc}a & & 1 & \\ & a & & \\ & & a & \\ & & a\end{array}\right)$, and

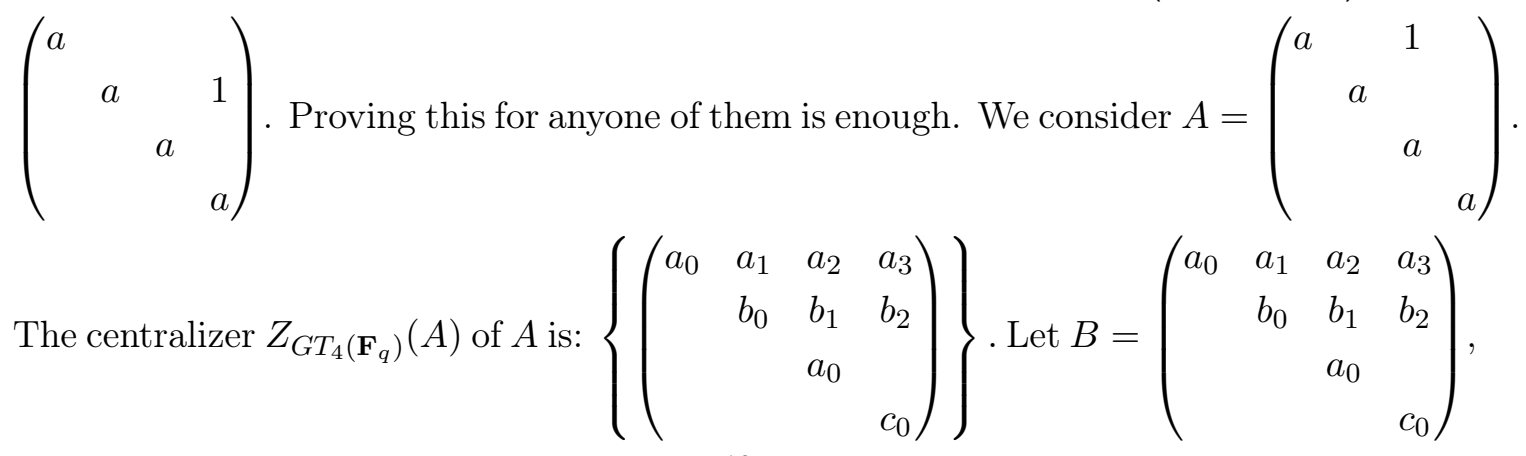
18 
and $B^{\prime}=\left(\begin{array}{cccc}a_{0} & a_{1}^{\prime} & a_{2}^{\prime} & a_{3}^{\prime} \\ & b_{0} & b_{1}^{\prime} & b_{2}^{\prime} \\ & & a_{0} & \\ & & & c_{0}\end{array}\right)$, be a conjugate of $B$ by $X=\left(\begin{array}{cccc}x_{0} & x_{1} & x_{2} & x_{3} \\ & y_{0} & y_{1} & y_{2} \\ & x_{0} & \\ & & z_{0}\end{array}\right)$. Denote $C=\left(\begin{array}{cc}b_{0} & b_{2} \\ & c_{0}\end{array}\right)$, and $Z=\left(\begin{array}{ll}y_{0} & y_{2} \\ & z_{0}\end{array}\right)$. Equating $X B=B^{\prime} X$, we have first $Z C=C^{\prime} Z$. Thus, we may take $C$ to be a canonical form from $G T_{2}\left(\mathbf{F}_{q}\right)$, and $Z \in Z_{G T_{2}\left(\mathbf{F}_{q}\right)}(C)$. With these, we have the following equations:

$$
\begin{aligned}
x_{0} \cdot\left(\begin{array}{ll}
a_{1} & a_{3}
\end{array}\right)+\left(\begin{array}{ll}
x_{1} & x_{3}
\end{array}\right)\left(\begin{array}{l}
C-a_{0} I_{2}
\end{array}\right) & =\left(\begin{array}{ll}
a_{1}^{\prime} & a_{3}^{\prime}
\end{array}\right) \cdot Z \\
y_{0} b_{1}+y_{1} a_{0} & =x_{0} b_{1}^{\prime}+y_{1} b_{0} \\
x_{0} a_{2}+x_{1} b_{1} & =x_{0} a_{2}^{\prime}+y_{1} a_{1}^{\prime}
\end{aligned}
$$

We have two main cases, under each of which there are subcases:

When $a_{0}$ is an eigenvalue of $C$ When $C=a_{0} I_{2}$ :, Equation 4.6 becomes:

$$
\left(\begin{array}{ll}
x_{0} a_{1} & x_{0} a_{3}
\end{array}\right)=\left(\begin{array}{ll}
y_{0} a_{1}^{\prime} & y_{2} a_{1}^{\prime}+z_{0} a_{3}^{\prime}
\end{array}\right)
$$

Equation 4.7 becomes $y_{0} b_{1}=x_{0} b_{1}^{\prime}$.

When $a_{1}=b_{1}=0$ : From Equation 4.9 we have $x_{0} a_{3}=z_{0} a_{3}^{\prime}$, and from Equation 4.8 $a_{2}^{\prime}=a_{2}$

We have two subcases:

When $a_{3}=0: B$ is reduced to $\left(\begin{array}{cccc}a_{0} & & a_{2} & \\ & a_{0} & & \\ & & a_{0} & \\ & & & a_{0}\end{array}\right)$, and $Z_{G T_{4}\left(\mathbf{F}_{q}\right)}(A, B)=Z_{G T_{4}\left(\mathbf{F}_{q}\right)}(A)$. $(A, B)$ is of type $A_{2}$, and there are $q(q-1)$ such branches.

When $a_{3} \neq 0$, choose $z_{0}$ so that $a_{3}^{\prime}=1$. $B$ is reduced to $\left(\begin{array}{llll}a_{0} & & a_{2} & 1 \\ & a_{0} & & \\ & a_{0} & \\ & & a_{0}\end{array}\right)$, with $Z_{G T_{4}\left(\mathbf{F}_{q}\right)}(A, B)=\left\{\left(\begin{array}{cccc}x_{0} & x_{1} & x_{2} & x_{3} \\ & y_{0} & y_{1} & y_{2} \\ & & x_{0} & \\ & & & x_{0}\end{array}\right)\right\}$. Now, this centralizer is not isomorphic to any known centralizer of a matrix in $G T_{4}\left(\mathbf{F}_{q}\right)$, and neither it is isomorphic to those of the three new types we encountered in the previous propositions. We have a new type $t N T_{3}$, and there are $q(q-1)$ such branches.

When $a_{1}=0$, and $b_{1} \neq 0$. In Equation 4.7 choose $y_{0}$ such that $b_{1}^{\prime}=1$. Then Equation 4.8 becomes $x_{0} a_{2}+x_{1}=x_{0} a_{2}^{\prime}$. Choose $x_{1}$ such that $a_{2}^{\prime}=0$. 
Here, when $a_{3}=0, B$ is reduced to $\left(\begin{array}{cccc}a_{0} & & & \\ & a_{0} & 1 & \\ & & a_{0} & \\ & & a_{0}\end{array}\right)$, with $Z_{G T_{4}\left(\mathbf{F}_{q}\right)}(A, B)=$ $\left\{\left(\begin{array}{llll}x_{0} & & x_{2} & x_{3} \\ & x_{0} & y_{1} & y_{2} \\ & & x_{0} & \\ & & & z_{0}\end{array}\right)\right\} .(A, B)$ is of type $t N T_{2}$, and there are $q-1$ such branches.

When $a_{3} \neq 0$, choose $z_{0}$ such that $a_{3}^{\prime}=1$. Thus $B$ is reduced to $\left(\begin{array}{ccccc}a_{0} & & & 1 \\ & a_{0} & 1 & \\ & & a_{0} & \\ & & & a_{0}\end{array}\right)$, with $Z_{G T_{4}\left(\mathbf{F}_{q}\right)}(A, B)=\left\{\left(\begin{array}{llll}x_{0} & & x_{2} & x_{3} \\ & x_{0} & y_{1} & y_{2} \\ & & x_{0} & \\ & & & x_{0}\end{array}\right)\right\} .(A, B)$ is of type $N R_{1}$, and there are $q-1$ such branches.

When $a_{1} \neq 0$, in Equation 4.9, choose $y_{0}$ such that $a_{1}^{\prime}=1$. Thus, on replacing $a_{1}$ with $a_{1}^{\prime}=1$ in Equation 4.9, we get $y_{0}=x_{0}$. In the same equation, choose $y_{2}$ such that $a_{3}^{\prime}=0$.

From Equation 4.7, we get $b_{1}^{\prime}=b_{1}$. Equation 4.8 becomes $x_{0} a_{2}+x_{1} b_{1}=x_{0} a_{2}^{\prime}+$ $y_{1}$. Choose $y_{1}$ such that $a_{2}^{\prime}=0$. Here, $B$ is reduced to $\left(\begin{array}{cccc}a_{0} & 1 & & \\ & a_{0} & b_{1} & \\ & a_{0} & \\ & & a_{0}\end{array}\right)$, with $Z_{G T_{4}\left(\mathbf{F}_{q}\right)}(A, B)=\left\{\left(\begin{array}{cccc}x_{0} & x_{1} & x_{2} & x_{3} \\ & x_{0} & b_{1} x_{1} & \\ & & x_{0} & \\ & & & z_{0}\end{array}\right)\right\} .(A, B)$ is of type $A_{7}$, and there are $q(q-1)$ such branches.

When $C=\left(\begin{array}{cc}a_{0} & 1 \\ & a_{0}\end{array}\right)$ : Equation 4.6 becomes

$$
\left(\begin{array}{ll}
x_{0} a_{1} & x_{0} a_{3}
\end{array}\right)+\left(\begin{array}{ll}
0 & x_{1}
\end{array}\right)=\left(\begin{array}{ll}
y_{0} a_{1}^{\prime} & y_{2} a_{1}^{\prime}+y_{0} a_{3}^{\prime}
\end{array}\right) .
$$

Choose $x_{1}$ such that $a_{3}^{\prime}=0$. Hence, on replacing $a_{3}$ by $a_{3}^{\prime}=0$ in the above equation, we have $x_{1}=a_{1}^{\prime} y_{2}$.

Equation 4.7 becomes $x_{0} b_{1}^{\prime}=y_{0} b_{1}$. 
When $a_{1}=b_{1}=0$, from Equation $4.8 a_{2}^{\prime}=a_{2}, B$ is reduced to $\left(\begin{array}{llll}a_{0} & & a_{2} & \\ & a_{0} & & 1 \\ & a_{0} & \\ & & a_{0}\end{array}\right)$, with $Z_{G T_{4}\left(\mathbf{F}_{q}\right)}(A, B)=\left\{\left(\begin{array}{llll}x_{0} & & x_{2} & x_{3} \\ & y_{0} & y_{1} & y_{2} \\ & & x_{0} & \\ & & & y_{0}\end{array}\right)\right\} .(A, B)$ is of type $A_{5}$. There are $q(q-1)$ such branches.

When $a_{1}=0$, and $b_{1} \neq 0$, we choose $y_{0}$ in Equation 4.7 so that $b_{1}^{\prime}=1$. So, Equation 4.8 becomes $x_{0} a_{2}=x_{0} a_{2}^{\prime}$, since $x_{1}=y_{2} a_{1}=0$. Hence $a_{2}^{\prime}=a_{2}$. So $B$ is reduced to $\left(\begin{array}{cccc}a_{0} & & a_{2} & \\ & a_{0} & 1 & 1 \\ & & a_{0} & \\ & & & a_{0}\end{array}\right)$, with $Z_{G T_{4}\left(\mathbf{F}_{q}\right)}(A, B)=\left\{\left(\begin{array}{cccc}x_{0} & & x_{2} & x_{3} \\ & x_{0} & y_{1} & y_{2} \\ & & x_{0} & \\ & & & x_{0}\end{array}\right)\right\}$. So, we have another $q(q-1)$ branches of type $N R_{1}$ here.

When $a_{1} \neq 0$, in Equation 4.6, choose $y_{0}$ so that $a_{1}^{\prime}=1$. So, $x_{1}=y_{2}$, and on replacing $a_{1}^{\prime}$ with $a_{1}$ in the same equation, we have $y_{0}=x_{0}$, and hence from Equation 4.7, $b_{1}^{\prime}=b_{1}$.

With these, Equation 4.8 becomes $x_{0} a_{2}+x_{1} b_{1}=x_{0} a_{2}^{\prime}+y_{1}$. Choose $y_{1}$ so that $a_{2}^{\prime}=0$. Thus $B$ is reduced to $\left(\begin{array}{cccc}a_{0} & 1 & & \\ & a_{0} & b_{1} & 1 \\ & & a_{0} & \\ & & & a_{0}\end{array}\right)$, with $Z_{G T_{4}\left(\mathbf{F}_{q}\right)}(A, B)=\left\{\left(\begin{array}{cccc}x_{0} & x_{1} & x_{2} & x_{3} \\ & x_{0} & b_{1} x_{1} & x_{1} \\ & & x_{0} & \\ & & & x_{0}\end{array}\right)\right\}$. By a routine check, one can see that this subgroup is commutative. Thus $(A, B)$ is of type $R_{1}$, and there are $q(q-1)$ such branches.

When $C=\left(\begin{array}{ll}a_{0} & \\ & b_{0}\end{array}\right), b_{0} \neq a_{0}$ : Here $Z=\left(\begin{array}{ll}y_{0} & \\ & z_{0}\end{array}\right)$. Equation 4.6 becomes

$$
\left(\begin{array}{ll}
x_{0} a_{1} & x_{0} a_{3}
\end{array}\right)+\left(\begin{array}{ll}
0 & \left(b_{0}-a_{0}\right) x_{3}
\end{array}\right)=\left(\begin{array}{ll}
y_{0} a_{1}^{\prime} & z_{0} a_{3}^{\prime}
\end{array}\right)
$$

And, Equation 4.7 becomes $y_{0} b_{1}=x_{0} b_{1}^{\prime}$.

Choose $x_{3}$ such that $a_{3}^{\prime}=0$.

When $a_{1}=b_{1}=0$, from Equation 4.8, we have $a_{2}^{\prime}=a_{2}$, and $B$ is reduced to

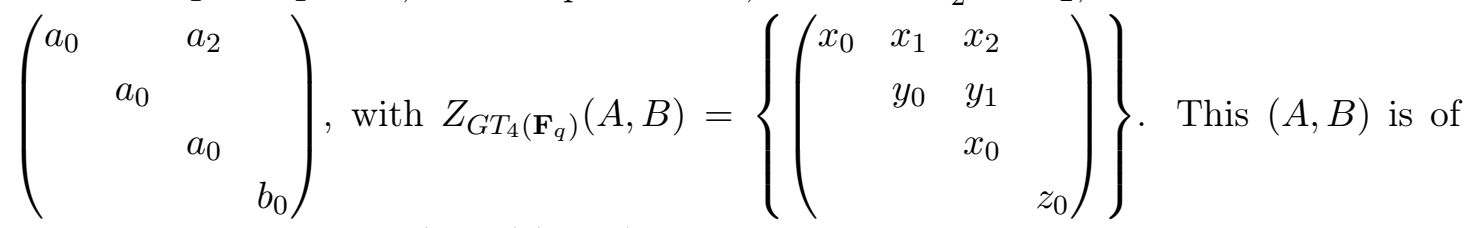
type $B_{4}$, and there are $q(q-1)(q-2)$ such branches.

When $a_{1}=0$, and $b_{1} \neq 0$. In Equation 4.7, choose $y_{0}$ so that $b_{1}^{\prime}=1$. And, Equation 4.8 becomes $x_{0} a_{2}+x_{1}=x_{0} a_{2}^{\prime}$. We choose $x_{1}$ so that $a_{2}^{\prime}=0$. Hence, $B$ is reduced to 


$$
\left(\begin{array}{cccc}
a_{0} & & & \\
& a_{0} & 1 & \\
& & a_{0} & \\
& & & b_{0}
\end{array}\right) \text {, with } Z_{G T_{4}\left(\mathbf{F}_{q}\right)}(A, B)=\left\{\left(\begin{array}{cccc}
x_{0} & & x_{2} & \\
& x_{0} & y_{1} & \\
& & x_{0} & \\
& & & z_{0}
\end{array}\right)\right\} \text {. By a routine check, }
$$

one can see that this subgroup is commutative. Thus $(A, B)$ is of type $R_{2}$, and there are $q(q-1)(q-2)$ such branches.

When $a_{1} \neq 0$, in Equation 4.6, choose $y_{0}$ so that $a_{1}^{\prime}=1$. Hence, on replacing $a_{1}$ by $a_{1}^{\prime}=1$ on both sides of Equation 4.11, we get $x_{0}=y_{0}$. Hence, Equation 4.7 becomes $x_{0} b_{1}=x_{0} b_{1}^{\prime}$, thus leaving use with $b_{1}^{\prime}=b_{1}$. Equation 4.8 becomes $x_{0} a_{2}+x_{1} b_{1}=y_{1}+x_{0} a_{2}^{\prime}$.

Thus, choose $y_{1}$ such that $a_{2}^{\prime}=0$. Hence $B$ is reduced to $\left(\begin{array}{cccc}a_{0} & 1 & & \\ & a_{0} & b_{1} & \\ & & a_{0} & \\ & & b_{0}\end{array}\right)$, with $Z_{G T_{4}\left(\mathbf{F}_{q}\right)}(A, B)=\left\{\left(\begin{array}{cccc}x_{0} & x_{1} & x_{2} & \\ & x_{0} & b_{1} x_{1} & \\ & & x_{0} & \\ & & & z_{0}\end{array}\right)\right\}$. By a routine check, one can see that this subgroup is commutative. Thus $(A, B)$ is of type $R_{2}$, and there are $q(q-1)(q-2)$ such branches.

When $C=\left(\begin{array}{cc}b_{0} & \\ & a_{0}\end{array}\right), a_{0} \neq b_{0}$ : Here too $Z=\left(\begin{array}{cc}y_{0} & \\ & z_{0}\end{array}\right)$. In this case, Equation 4.6 is reduced to $\left(\begin{array}{ll}x_{0} a_{1} & x_{0} a_{3}\end{array}\right)+\left(\begin{array}{ll}\left(b_{0}-a_{0}\right) x_{1} & 0\end{array}\right)=\left(\begin{array}{ll}y_{0} a_{1}^{\prime} & z_{0} a_{3}^{\prime}\end{array}\right)$. Choose $x_{1}$ so that $a_{1}^{\prime}=0$.

Equation 4.7 becomes $y_{0} b_{1}+y_{1} a_{0}=y_{1} b_{0}+x_{0} b_{1}^{\prime}$. As $a_{0} \neq b_{0}$, choose $y_{1}$ such that $b_{1}^{\prime}=0$. With these, Equation 4.8 becomes $x_{0} a_{2}=x_{0} a_{2}^{\prime}$, thus leaving us with $a_{2}^{\prime}=a_{2}$.

Now, we are left to deal with $a_{3}$.

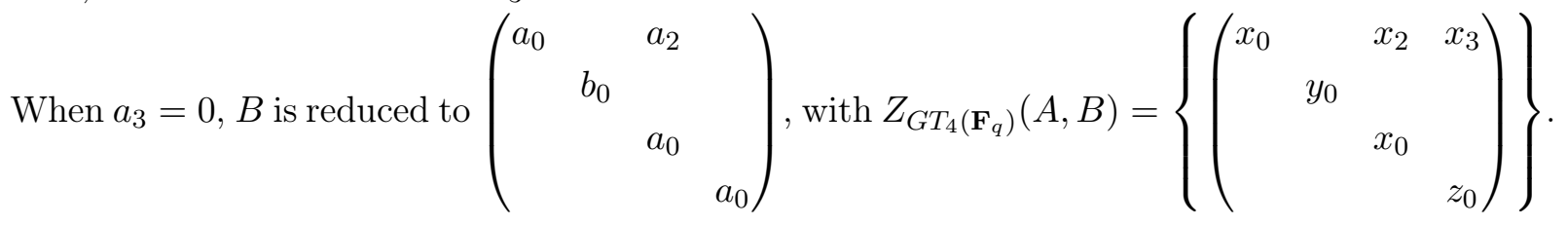

This subgroup is isomorphic to the subgroup $\left\{\left(\begin{array}{llll}y_{0} & & & \\ & x_{0} & x_{2} & x_{3} \\ & x_{0} & \\ & & z_{0}\end{array}\right)\right\}$, which is the centralizer of a matrix of type $B_{3}$. Hence, we have $q(q-1)(q-2)$ branches of type $B_{3}$. 


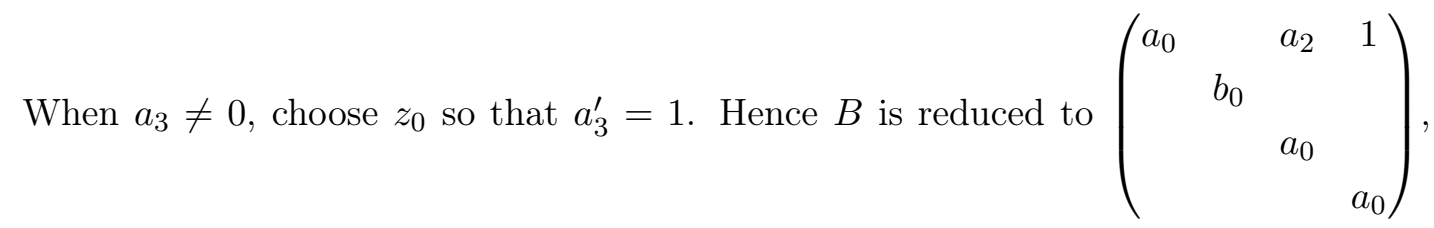
with $Z_{G T_{4}\left(\mathbf{F}_{q}\right)}(A, B)=\left\{\left(\begin{array}{cccc}x_{0} & & x_{2} & x_{3} \\ & y_{0} & & \\ & & x_{0} & \\ & & & x_{0}\end{array}\right)\right\}$. By a routine check, one can see that this subgroup is commutative. Thus $(A, B)$ is of type $R_{2}$, and there are $q(q-1)(q-2)$ such branches. With this we have looked at all the cases, when $a_{0}$ is an eigenvalue of $C$.

When $a_{0}$ is not an eigenvalue oc $C$ : When $a_{0}$ is not an eigenvalue of $C, C-a_{0} I_{2}=$ $\left(\begin{array}{cc}b_{0}-a_{0} & b_{2} \\ & c_{0}-a_{0}\end{array}\right)$, with $b_{0}-a_{0} \neq 0$, and $c_{0}-a_{0} \neq 0$. Hence, in equation 4.6, we can choose $\left(\begin{array}{ll}x_{1} & x_{3}\end{array}\right)$ such that $a_{1}^{\prime}=0$, and $a_{3}^{\prime}=0$. In Equation 4.7 choose $y_{1}$ so that $b_{1}^{\prime}=0$. Hence Equation 4.8 becomes $x_{0} a_{2}=x_{0} a_{2}^{\prime}$. Therefore $a_{2}^{\prime}=a_{2}$.

On replacing $a_{1}$ and $a_{3}$ by 0 in Equation 4.6, we get $x_{1}=x_{3}=0$, and on replacing $b_{1}$ by 0 in Eqaution 4.7, we get $y_{1}=0$. Now, we can look at the various cases of $C$.

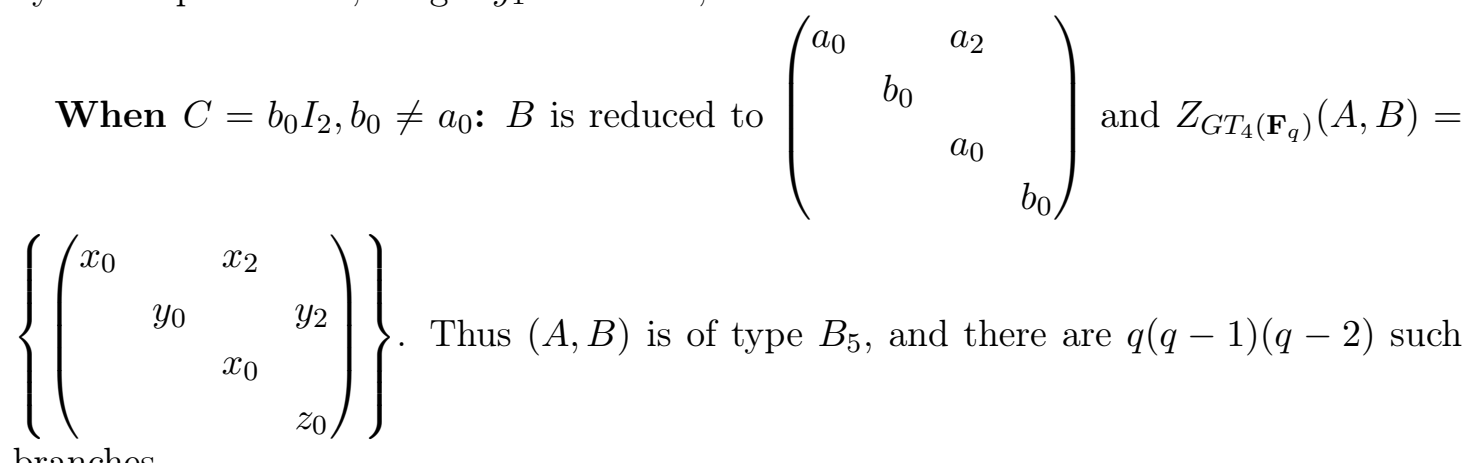
branches.

$$
\begin{aligned}
& \text { When } C=\left(\begin{array}{cc}
b_{0} & 1 \\
& b_{0}
\end{array}\right), b_{0} \neq a_{0}: B \text { is reduced to }\left(\begin{array}{cccc}
a_{0} & & a_{2} & \\
& b_{0} & & 1 \\
& a_{0} & \\
& & b_{0}
\end{array}\right) \text { and } Z_{G T_{4}\left(\mathbf{F}_{q}\right)}(A, B)= \\
& \left\{\left(\begin{array}{llll}
x_{0} & & x_{2} & \\
& y_{0} & & y_{2} \\
& & x_{0} & \\
& & & y_{0}
\end{array}\right)\right\} \text {. Thus }(A, B) \text { is of type } R_{3} \text {, and there are } q(q-1)(q-2) \text { such }
\end{aligned}
$$




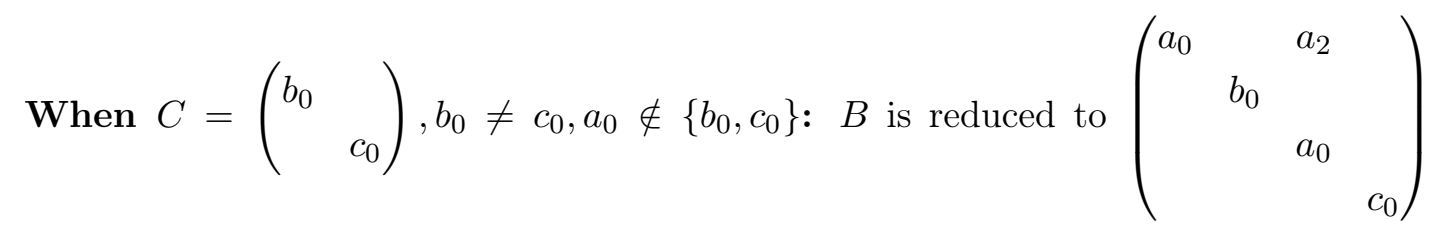
and $Z_{G T_{4}\left(\mathbf{F}_{q}\right)}(A, B)=\left\{\left(\begin{array}{cccc}x_{0} & & x_{2} & \\ & y_{0} & & \\ & & x_{0} & \\ & & & z_{0}\end{array}\right)\right\}$. Thus $(A, B)$ is of type $R_{4}$, and there are $q(q-1)(q-2)(q-3)$ such branches.

Adding up the branches of type $N R_{1}$, we have a total of $q-1+q(q-1)=q^{2}-1$ branches of type $R_{1}$.

Proposition 4.5. For a matrix of type $A_{3}$, the branching rules are in the table below:

\begin{tabular}{c|c||c|c}
\hline Branch & No. of Branches & Branch & No. of Branches \\
\hline$A_{3}$ & $q(q-1)$ & $R_{1}$ & $q^{2}-1$ \\
$A_{5}$ & $q(q-1)$ & $R_{2}$ & $2\left(q^{2}-1\right)(q-2)$ \\
$A_{8}$ & $2 q(q-1)$ & $R_{3}$ & $\left(q^{2}-q\right)(q-2)$ \\
$A_{9}$ & $q(q-1)$ & $R_{4}$ & $\left(q^{2}-q\right)(q-2)(q-3)$ \\
$B_{4}$ & $2\left(q^{2}-q\right)(q-1)$ & $t N T_{3}$ & $2(q-1)$ \\
$B_{5}$ & $\left(q^{2}-q\right)(q-1)$ & $t N T_{4}$ & $q-1$. \\
\hline
\end{tabular}

A new type $t N T_{4}$ appears here, whose centralizer is $\left\{\left(\begin{array}{cccc}x_{0} & x_{1} & x_{2} & x_{3} \\ & x_{0} & y_{1} & y_{2} \\ & x_{0} & x_{1} \\ & & x_{0}\end{array}\right) \mid x_{0} \neq 0\right\}$.

Proof. A matrix of type $A_{3}$ has the canonical form $A=\left(\begin{array}{cccc}a & & & 1 \\ & a & & \\ & & a & \\ & & a\end{array}\right)$. Then we have $Z_{G T_{4}\left(\mathbf{F}_{q}\right)}(A)=\left\{\left(\begin{array}{ccc}a_{0} & { }^{t} \vec{b} & a_{1} \\ & C & \vec{d} \\ & & a_{0}\end{array}\right) \begin{array}{c}{ }^{t} \vec{b}=\left(b_{1} b_{2}\right) \in M_{1 \times 2}\left(\mathbf{F}_{q}\right) \\ \vec{d}=\left(\begin{array}{c}d_{1} \\ d_{2}\end{array}\right) \in M_{2 \times 1}\left(\mathbf{F}_{q}\right)\end{array}\right\}$. Let $B=\left(\begin{array}{ccc}a_{0} & t \vec{b} & a_{1} \\ & C & \vec{d} \\ & & a_{0}\end{array}\right)$, and $B^{\prime}=\left(\begin{array}{ccc}a_{0} & { }^{t} \vec{b}^{\prime} & a_{1}^{\prime} \\ & C^{\prime} & \overrightarrow{d^{\prime}} \\ & & a_{0}\end{array}\right)=X B A^{-1}$, where $X=\left(\begin{array}{ccc}x_{0} & t \vec{y} & x_{1} \\ & Z & \vec{w} \\ & & x_{0}\end{array}\right) . X B=B^{\prime} X$ leads to firstly, $Z C=C^{\prime} Z$, hence we shall take $C$ to be a canonical conjugacy class representative 
in $G T_{2}\left(\mathbf{F}_{q}\right)$, and $Z \in Z_{G T_{2}\left(\mathbf{F}_{q}\right)}(C)$. Then we have the following set of equations:

$$
\begin{aligned}
x_{0}{ }^{t} \vec{b}+{ }^{t} \vec{y} \cdot\left(C-a_{0} I_{2}\right) & ={ }^{t} \vec{b}^{\prime} \cdot Z \\
Z \cdot \vec{d}+\left(a_{0} I_{2}-C\right) \vec{w} & =x_{0} \vec{d}^{\prime} \\
x_{0} a_{1}+y_{1} d_{1}+y_{2} d_{2} & =x_{0} a_{1}^{\prime}+b_{1}^{\prime} w_{1}+b_{2}^{\prime} w_{2}
\end{aligned}
$$

\section{When $a_{0}$ is an eigenvalue of $C$ :}

When $\vec{b}=\vec{d}=\overrightarrow{0}$ :

Here, Equation 4.12 becomes ${ }^{t} \vec{y}\left(C-a_{0} I_{2}\right)={ }^{t} \overrightarrow{0}$, Equation 4.13 becomes $\left(a_{0} I_{2}-\right.$ C) $\vec{w}=\overrightarrow{0}$, and Equation 4.14 becomes $x_{0} a_{1}=x_{0} a_{1}^{\prime}$. Hence we have $a_{1}^{\prime}=a_{1}$.

When $C=a_{0} I_{2}$ : Equations 4.12 and 4.13 are void. Hence $B$ is reduced to $\left(\begin{array}{llll}a_{0} & & & a_{1} \\ & & & \\ & a_{0} & & \\ & & a_{0} & \\ & & & a_{0}\end{array}\right)$,

and $Z_{G T_{4}\left(\mathbf{F}_{q}\right)}(A, B)=Z_{G T_{4}\left(\mathbf{F}_{q}\right)}(A) . \quad(A, B)$ is of type $A_{2}$, and there are $q(q-1)$ such branches.

When $C=\left(\begin{array}{cc}a_{0} & 1 \\ & a_{0}\end{array}\right)$ : Here Equation 4.12 becomes: $\left(\begin{array}{ll}0 & y_{1}\end{array}\right)=\left(\begin{array}{ll}0 & 0\end{array}\right)$, and Equation 4.13 becomes $\left(\begin{array}{c}-w_{2} \\ 0\end{array}\right)=\left(\begin{array}{l}0 \\ 0\end{array}\right)$. Thus $y_{1}=0$, and $w_{2}=0 . B$ is reduced to

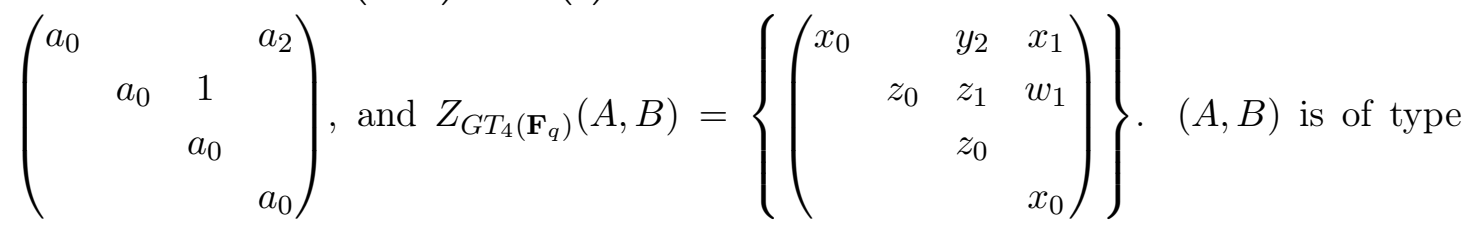
$A_{5}$, and there are $q(q-1)$ such branches.

When $C=\left(\begin{array}{cc}a_{0} & \\ & c_{0}\end{array}\right), a_{0} \neq c_{0}$ : Here Equation 4.12 becomes: $\left(\begin{array}{ll}0 & \left.y_{2}\left(c_{0}-a_{0}\right)\right)=\end{array}\right.$ $\left(\begin{array}{ll}0 & 0\end{array}\right)$, and Equation 4.13 becomes $\left(\begin{array}{l}\left(a_{0}-c_{0}\right) w_{2}\end{array}\right)=\left(\begin{array}{l}0 \\ 0\end{array}\right)$. Thus $y_{2}=0$, and $w_{2}=0$. B is reduced to $\left(\begin{array}{cccc}a_{0} & & & a_{2} \\ & a_{0} & & \\ & & c_{0} & \\ & & & a_{0}\end{array}\right)$, and $Z_{G T_{4}\left(\mathbf{F}_{q}\right)}(A, B)=\left\{\left(\begin{array}{cccc}x_{0} & y_{1} & & x_{1} \\ & z_{0} & & w_{1} \\ & z_{2} & \\ & & x_{0}\end{array}\right)\right\} .(A, B)$ is of type $B_{4}$, and there are $q(q-1)(q-2)$ such branches.

When $C=\left(\begin{array}{ll}b_{0} & \\ & a_{0}\end{array}\right), a_{0} \neq b_{0}$ : Here Equation 4.12 becomes: $\left(\begin{array}{ll}\left(b_{0}-a_{0}\right) y_{1} & 0\end{array}\right)=$ $\left(\begin{array}{ll}0 & 0\end{array}\right)$, and Equation 4.13 becomes $\left(\begin{array}{c}\left(b_{0}-a_{0}\right) w_{1} \\ 0 \\ 25\end{array}\right)=\left(\begin{array}{l}0 \\ 0\end{array}\right)$. Thus $y_{1}=0$, and $w_{1}=0$. 


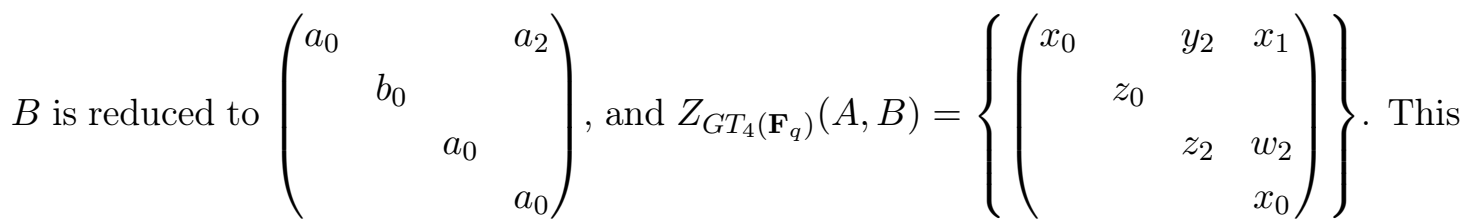
$(A, B)$ too is of type $B_{4}$, and there are $q(q-1)(q-2)$ such branches.

When $(\vec{b}, \vec{d}) \neq(\overrightarrow{0}, \overrightarrow{0})$.

When $C=a_{0} I_{2}$ : Equation 4.12 becomes:

$$
\left(\begin{array}{ll}
x_{0} b_{1} & x_{0} b_{2}
\end{array}\right)=\left(\begin{array}{ll}
z_{0} b_{1}^{\prime} & z_{1} b_{1}^{\prime}+z_{2} b_{2}
\end{array}\right)
$$

and Equation 4.13 becomes:

$$
\left(\begin{array}{c}
z_{0} d_{1}+z_{1} d_{2} \\
z_{2} d_{2}
\end{array}\right)=\left(\begin{array}{c}
x_{0} d_{1}^{\prime} \\
x_{0} d_{2}^{\prime}
\end{array}\right) .
$$

When $b_{1}=0$ and $b_{2} \neq 0$. In Equation 4.15 choose $z_{2}$ so that $b_{2}^{\prime}=1$. Hence, on replacing $b_{2}$ by $b_{2}^{\prime}=1$ in Equation 4.15, we get $x_{0}=z_{2}$. Hence in Equation 4.16, $x_{0} d_{2}^{\prime}=x_{0} d_{2}$. Thus $d_{2}=d_{2}^{\prime}$.

Here, if $d_{2}=0$, in Equation 4.16, we have $x_{0} d_{1}^{\prime}=z_{0} d_{1}$. When $d_{1}=0$; Equation 4.14 becomes $x_{0} a_{1}=x_{0} a_{1}^{\prime}+w_{2}$. Choose $w_{2}$ so that $a_{1}^{\prime}=0$. Hence $B$ is reduced to $\left(\begin{array}{cccc}a_{0} & & 1 & \\ & a_{0} & & \\ & & a_{0} & \\ & & & a_{0}\end{array}\right)$, and $Z_{G T_{4}\left(\mathbf{F}_{q}\right)}(A, B)=\left\{\left(\begin{array}{cccc}x_{0} & y_{1} & y_{2} & x_{1} \\ & z_{0} & z_{1} & w_{1} \\ & & x_{0} & \\ & & & x_{0}\end{array}\right)\right\} \cdot(A, B)$ is of type $t N T_{3}$, and there are $q-1$ such branches.

When $d_{1} \neq 0$, choose $z_{0}$ so that $d_{1}^{\prime}=1$. Then Equation 4.14 becomes $x_{0} a_{1}+y_{1}=$ $x_{0} a_{1}^{\prime} w_{2}$. Choose $w_{2}$ such that $a_{1}^{\prime}=0$. With these, $B$ is reduced to: $\left(\begin{array}{cccc}a_{0} & & & \\ & & & \\ & a_{0} & & 1 \\ & a_{0} & \\ & & a_{0}\end{array}\right)$, and $Z_{G T_{4}\left(\mathbf{F}_{q}\right)}(A, B)=\left\{\left(\begin{array}{cccc}x_{0} & y_{1} & y_{2} & x_{1} \\ & x_{0} & z_{1} & w_{1} \\ & & x_{0} & y_{1} \\ & & & x_{0}\end{array}\right)\right\}$. Now, this is a centralizer we have not seen so far. Thus we have a new type, $t N T_{4}$. There are $q-1$ such branches.

When $d_{2} \neq 0$, in Equation 4.16, choose $z_{1}$ so that $d_{1}^{\prime}=0$. Equation 4.14 becomes $x_{0} a_{1}+$ $y_{2} d_{2}=x_{0} a_{1}^{\prime}+w_{2}$. Choose $w_{2}$ such that $a_{1}^{\prime}=0$. So, $B$ is reduced to $\left(\begin{array}{cccc}a_{0} & & 1 & \\ & a_{0} & & \\ & a_{0} & d_{2} \\ & & a_{0}\end{array}\right)$ 
and $Z_{G T_{4}\left(\mathbf{F}_{q}\right)}(A, B)=\left\{\left(\begin{array}{cccc}x_{0} & y_{1} & y_{2} & x_{1} \\ & z_{0} & & w_{1} \\ & & x_{0} & d_{2} y_{2} \\ & & & x_{0}\end{array}\right)\right\} .(A, B)$ is of type $A_{8}$, and there are $(q-1)^{2}$ such branches.

When $b_{1}=b_{2}=d_{2}=0$. Here $d_{1} \neq 0$. Choose $z_{0}$ so that $d_{1}^{\prime}=1$. Then, in Equation 4.14, we have $x_{0} a_{1}+y_{1}=x_{0} a_{1}^{\prime}$. Choose $y_{1}$ so that $a_{1}^{\prime}=0$. Thus $B$ is reduced to: $\left\{\begin{array}{cccc}a_{0} & & & \\ & a_{0} & & 1 \\ & & a_{0} & \\ & & & a_{0}\end{array}\right)$, and $Z_{G T_{4}\left(\mathbf{F}_{q}\right)}(A, B)=\left\{\left(\begin{array}{cccc}x_{0} & & y_{2} & x_{1} \\ & x_{0} & z_{1} & w_{1} \\ & & z_{2} & w_{2} \\ & & x_{0}\end{array}\right)\right\} .(A, B)$ is of type $t N T_{3}$. There are $q-1$ such branches.

When $b_{1}=b_{2}=0$, and $d_{2} \neq 0$. In Equation 4.16, choose $z_{2}$ such that $d_{2}^{\prime}=1$, and in the same equation, choose $z_{1}$ so that $d_{1}^{\prime}=0$. With these, Equation 4.14 becomes $x_{0} a_{1}+y_{2}=x_{0} a_{1}^{\prime}$. Choose $y_{2}$ such that $a_{1}^{\prime}=0$. Thus $B$ is reduced to: $\left(\begin{array}{cccc}a_{0} & & & \\ & a_{0} & & \\ & & & \\ & a_{0} & 1 \\ & & a_{0}\end{array}\right)$, and $Z_{G T_{4}\left(\mathbf{F}_{q}\right)}(A, B)=\left\{\left(\begin{array}{cccc}x_{0} & y_{1} & & x_{1} \\ & z_{0} & & w_{1} \\ & & x_{0} & w_{2} \\ & & & x_{0}\end{array}\right)\right\}$. This too is of type $A_{8}$. There are $q-1$ such branches.

When $b_{1} \neq 0$. In Equation 4.15 , choose $z_{0}$ so that $b_{1}^{\prime}=1$, and choose $z_{1}$ so that $b_{2}^{\prime}=0$. On replacing $b_{1}$ with $b_{1}^{\prime}=1$, and $b_{2}$ with $b_{2}^{\prime}=0$ in Equation 4.15 , we get $z_{0}=x_{0}$, and $z_{1}=0$. Putting these in equation 4.16 leaves us with $d_{1}^{\prime}=d_{1}$ and $z_{2} d_{2}=x_{0} d_{2}^{\prime}$.

With these, Equation 4.14 is reduced to $x_{0} a_{1}+d_{1} y_{1}=x_{0} a_{1}^{\prime}+w_{1}$. Choose $w_{1}$ so that $a_{1}^{\prime}=0$.

When $d_{2}=0, B$ is reduced to $\left(\begin{array}{cccc}a_{0} & 1 & & \\ & a_{0} & & d_{1} \\ & & a_{0} & \\ & & & a_{0}\end{array}\right)$, and $Z_{G T_{4}\left(\mathbf{F}_{q}\right)}(A, B)=\left\{\left(\begin{array}{cccc}x_{0} & y_{1} & y_{2} & x_{1} \\ & x_{0} & & d_{1} y_{1} \\ & & z_{2} & w_{2} \\ & & x_{0}\end{array}\right)\right\}$.

This too is of type $A_{8}$. There are $q(q-1)$ such branches.

When $d_{2} \neq 0$, in Equation 4.16, choose $z_{2}$ so that $d_{2}^{\prime}=1$. With these Equation 4.14 becomes $x_{0} a_{1}+y_{1} d_{1}+y_{2}=x_{0} a_{1}^{\prime}+w_{1}$. Choose $w_{1}$ such that $a_{1}^{\prime}=0$. Thus $B$ is reduced 
to $\left(\begin{array}{cccc}a_{0} & 1 & & \\ & a_{0} & & d_{1} \\ & a_{0} & 1 \\ & & & a_{0}\end{array}\right)$, and $Z_{G T_{4}\left(\mathbf{F}_{q}\right)}(A, B)=\left\{\left(\begin{array}{cccc}x_{0} & y_{1} & y_{2} & x_{1} \\ & x_{0} & & y_{2}+d_{1} y_{1} \\ & x_{0} & w_{2} \\ & & x_{0}\end{array}\right)\right\}$. This is of type $A_{9}$. There are $q(q-1)$ such branches.

So, with these, we are done with all the cases, when $C=a_{0} I_{2}$.

When $C=\left(\begin{array}{cc}a_{0} & 1 \\ & a_{0}\end{array}\right)$ : Here $Z=\left(\begin{array}{cc}z_{0} & z_{1} \\ & z_{0}\end{array}\right)$. Equation 4.12 becomes:

$$
\left(\begin{array}{ll}
x_{0} b_{1} & x_{0} b_{2}
\end{array}\right)+\left(\begin{array}{ll}
0 & y_{1}
\end{array}\right)=\left(\begin{array}{ll}
z_{0} b_{1}^{\prime} & z_{1} b_{1}^{\prime}+z_{0} b_{2}^{\prime}
\end{array}\right)
$$

Choose $y_{1}$ such that $b_{2}^{\prime}=0$. On substituting $b_{2}$ with $b_{2}^{\prime}=0$ in the above equation, we have $y_{1}=z_{1} b_{1}^{\prime}$.

Similarly, Equation 4.13 becomes

$$
\left(\begin{array}{c}
z_{0} d_{1}+z_{1} d_{2} \\
z_{0} d_{2}
\end{array}\right)+\left(\begin{array}{c}
-w_{2} \\
0
\end{array}\right)=\left(\begin{array}{l}
x_{0} b_{1}^{\prime} \\
x_{0} b_{2}^{\prime}
\end{array}\right) .
$$

Choose $w_{2}$ such that $d_{1}^{\prime}=0$. On substituting $d_{1}$ with $d_{1}^{\prime}=0$ in the above equation, we have $w_{2}=d_{2} z_{1}$.

When $b_{1} \neq 0$, choose $z_{0}$ so that $b_{1}^{\prime}=1$. Then, on substituting $b_{1}$ with $b_{1}^{\prime}=1$ in Equation 4.12, we get $z_{0}=x_{0}$, and thus $d_{2}^{\prime}=d_{2}$. With these, Equation 4.14 becomes $x_{0} a_{1}+y_{2} d_{2}=x_{0} a_{1}^{\prime}+w_{1}$. Choose $w_{1}$ such that $a_{1}^{\prime}=0$. Thus, $B$ is reduced to $\left(\begin{array}{cccc}a_{0} & 1 & & \\ & a_{0} & 1 & \\ & & a_{0} & d_{2} \\ & & & a_{0}\end{array}\right)$, and $Z_{G T_{4}\left(\mathbf{F}_{q}\right)}(A, B)=\left\{\left(\begin{array}{cccc}x_{0} & z_{1} & y_{2} & x_{1} \\ & x_{0} & z_{1} & d_{2} y_{2} \\ & & x_{0} & d_{2} z_{1} \\ & & & x_{0}\end{array}\right)\right\}$. This $(A, B)$ is of type $R_{1}$, and there are $q(q-1)$ such branches.

When $b_{1}=0$, and $d_{2} \neq 0 y_{1}=0$. In Equation 4.13, choose $z_{0}$ so that $d_{2}^{\prime}=1$. With these, Equation 4.14 becomes $x_{0} a_{1}+y_{2}=x_{0} a_{1}^{\prime}$. Choose $y_{2}$ so that $a_{1}^{\prime}=0$. Thus, $B$ is reduced to $\left(\begin{array}{cccc}a_{0} & & & \\ & a_{0} & 1 & \\ & & a_{0} & 1 \\ & & & a_{0}\end{array}\right)$, and $Z_{G T_{4}\left(\mathbf{F}_{q}\right)}(A, B)=\left\{\left(\begin{array}{cccc}x_{0} & & & x_{1} \\ & x_{0} & z_{1} & w_{1} \\ & & x_{0} & z_{1} \\ & & x_{0}\end{array}\right)\right\}$. By a routine check, one can see that this is commutative. Thus $(A, B)$ is of type $R_{1}$, and there are $q-1$ such branches.

$$
\begin{gathered}
\text { When } C=\left(\begin{array}{ll}
a_{0} & \\
& b_{0}
\end{array}\right), b_{0} \neq a_{0} \text { : Here } Z=\left(\begin{array}{ll}
z_{0} & \\
& z_{2}
\end{array}\right) \text {. Equation } 4.12 \text { becomes : } \\
\left(\begin{array}{ll}
x_{0} b_{1} & x_{0} b_{2}
\end{array}\right)+\left(\begin{array}{cc}
0 & \left(b_{0}-a_{0}\right) y_{2} \\
28
\end{array}\right)=\left(\begin{array}{ll}
z_{0} b_{1}^{\prime} & z_{2} b_{2}^{\prime}
\end{array}\right)
\end{gathered}
$$


As $b_{0}-a_{0} \neq 0$, choose $y_{2}$ such that $b_{2}^{\prime}=0$. Hence, on replacing $b_{2}$ by $b_{2}^{\prime}=0$ in the above equation, we get $y_{2}=0$.

Similarly Equation 4.13 becomes:

$$
\left(\begin{array}{ll}
z_{0} d_{1} & z_{2} d_{2}
\end{array}\right)+\left(\begin{array}{c}
0 \\
\left(a_{0}-b_{0}\right) w_{2}
\end{array}\right)=\left(\begin{array}{ll}
x_{0} d_{1}^{\prime} & x_{0} d_{2}^{\prime}
\end{array}\right)
$$

Choose $w_{2}$ so that $d_{2}^{\prime}=0$. So, if we replace $d_{2}$ by $d_{2}^{\prime}=0$ in the above equation, we have $w_{2}=0$.

When $b_{1}=0$ and $d_{1} \neq 0$, choose $z_{0}$ so that $d_{1}^{\prime}=1$. With these, Equation 4.14 becomes $x_{0} a_{1}+y_{1}=x_{0} a_{1}^{\prime}$. Choose $y_{1}$ so that $a_{1}^{\prime}=0 . B$ is thus reduced to $\left(\begin{array}{llll}a_{0} & & & \\ & a_{0} & & 1 \\ & b_{0} & \\ & & a_{0}\end{array}\right)$, and $Z_{G T_{4}\left(\mathbf{F}_{q}\right)}(A, B)=\left\{\left(\begin{array}{llll}x_{0} & & & x_{1} \\ & x_{0} & & w_{1} \\ & & z_{2} & \\ & & & x_{0}\end{array}\right)\right\}$. By a routine check, we can see that this centralizer is commutative. Thus $(A, B)$ is of type $R_{2}$, and there are $(q-1)(q-2)$ such branches.

When $b_{1} \neq 0$. in Equation 4.12 for this $C$, choose $z_{0}$ so that $b_{1}^{\prime}=1$. Thus on substituting $b_{1}$ with $b_{1}^{\prime}=1$ in the same, we get $z_{0}=x_{0}$. Hence, from Equation 4.13 for this case, we have $d_{1}^{\prime}=d_{1}$. With these Equation 4.14 becomes $x_{0} a_{1}+d_{1} y_{1}=$ $x_{0} a_{1}^{\prime}+w_{1}$. Choose $w_{1}$ so that $a_{1}^{\prime}=0$. Hence $B$ is reduced to $\left(\begin{array}{cccc}a_{0} & 1 & & \\ & a_{0} & & d_{1} \\ & & b_{0} & \\ & & a_{0}\end{array}\right)$ and $Z_{G T_{4}\left(\mathbf{F}_{q}\right)}(A, B)=\left\{\left(\begin{array}{cccc}x_{0} & y_{1} & & x_{1} \\ & x_{0} & & d_{1} y_{1} \\ & & z_{2} & \\ & & & x_{0}\end{array}\right)\right\}$. Easy to see that this centralizer too is commutative. Thus $(A, B)$ is of type $R_{2}$, and there are $q(q-1)(q-2)$ such branches.

$$
\begin{gathered}
\text { When } C=\left(\begin{array}{ll}
b_{0} & \\
& a_{0}
\end{array}\right), b_{0} \neq a_{0} \text { : Here } Z=\left(\begin{array}{ll}
z_{0} & \\
& z_{2}
\end{array}\right) \text {. Equation } 4.12 \text { becomes : } \\
\left(\begin{array}{ll}
x_{0} b_{1} & x_{0} b_{2}
\end{array}\right)+\left(\left(\begin{array}{ll}
\left.b_{0}-a_{0}\right) y_{1} & 0
\end{array}\right)=\left(\begin{array}{ll}
z_{0} b_{1}^{\prime} & z_{2} b_{2}^{\prime}
\end{array}\right)\right.
\end{gathered}
$$

As $b_{0}-a_{0} \neq 0$, choose $y_{1}$ such that $b_{1}^{\prime}=0$. Hence, on replacing $b_{1}$ by $b_{1}^{\prime}=0$ in the above equation, we get $y_{1}=0$. 
Similarly Equation 4.13 becomes $\left(\begin{array}{ll}z_{0} d_{1} & z_{2} d_{2}\end{array}\right)+\left(\begin{array}{c}\left(a_{0}-b_{0}\right) w_{1} \\ 0\end{array}\right)=\left(\begin{array}{ll}x_{0} d_{1}^{\prime} & x_{0} d_{2}^{\prime}\end{array}\right)$. Choose $w_{1}$ so that $d_{1}^{\prime}=0$. So, if we replace $d_{1}$ by $d_{1}^{\prime}=0$ in the above equation, we have $w_{1}=0$.

When $b_{2}=0$ and $d_{2} \neq 0$, choose $z_{2}$ so that $d_{2}^{\prime}=1$. With these, Equation 4.14 becomes $x_{0} a_{1}+y_{2}=x_{0} a_{1}^{\prime}$. Choose $y_{2}$ so that $a_{1}^{\prime}=0 . B$ is thus reduced to $\left(\begin{array}{cccc}a_{0} & & & \\ & b_{0} & & \\ & & a_{0} & 1 \\ & & a_{0}\end{array}\right)$, and $Z_{G T_{4}\left(\mathbf{F}_{q}\right)}(A, B)=\left\{\left(\begin{array}{llll}x_{0} & & & x_{1} \\ & z_{0} & & \\ & & x_{0} & w_{2} \\ & & & x_{0}\end{array}\right)\right\}$. By a routine check, we can see that this centralizer is commutative. This $(A, B)$ is of type $R_{2}$, and there are $(q-1)(q-2)$ such branches.

When $b_{2} \neq 0$, in Equation 4.12 for this $C$, choose $z_{2}$ so that $b_{2}^{\prime}=1$. Thus on substituting $b_{2}$ with $b_{2}^{\prime}=1$ in the same, we get $z_{2}=x_{0}$. Hence, from Equation 4.13 for this case, we have $d_{2}^{\prime}=d_{2}$. With these Equation 4.14 becomes $x_{0} a_{1}+d_{2} y_{2}=$ $x_{0} a_{1}^{\prime}+w_{2}$. Choose $w_{2}$ so that $a_{1}^{\prime}=0$. Hence $B$ is reduced to $\left(\begin{array}{cccc}a_{0} & 1 & & \\ & a_{0} & & d_{1} \\ & b_{0} & \\ & & a_{0}\end{array}\right)$ and $Z_{G T_{4}\left(\mathbf{F}_{q}\right)}(A, B)=\left\{\left(\begin{array}{cccc}x_{0} & & y_{2} & x_{1} \\ & z_{0} & & \\ & & x_{0} & d_{2} y_{2} \\ & & & x_{0}\end{array}\right)\right\}$. Easy to see that this centralizer is commutative. This $(A, B)$ too is of type $R$, and there are $q(q-1)(q-2)$ such branches.

With these, we have covered all the subcases under the case of $a_{0}$ being an eigenvalue of $C$.

When $a_{0}$ is not an eigenvalue of $C$ : In this case $C-a_{0} I_{2}$ is invertible. Hence, in Equation 4.12, choose $y_{1}, y_{2}$ so that $b_{1}^{\prime}=b_{2}^{\prime}=0$. Similarly, in Equation 4.13, choose $w_{1}, w_{2}$ so that $d_{1}^{\prime}=d_{2}^{\prime}=0$.

So, Equation 4.14 becomes $x_{0} a_{1}=x_{0} a_{1}^{\prime}$, thus $a_{1}^{\prime}=a_{1}$. 


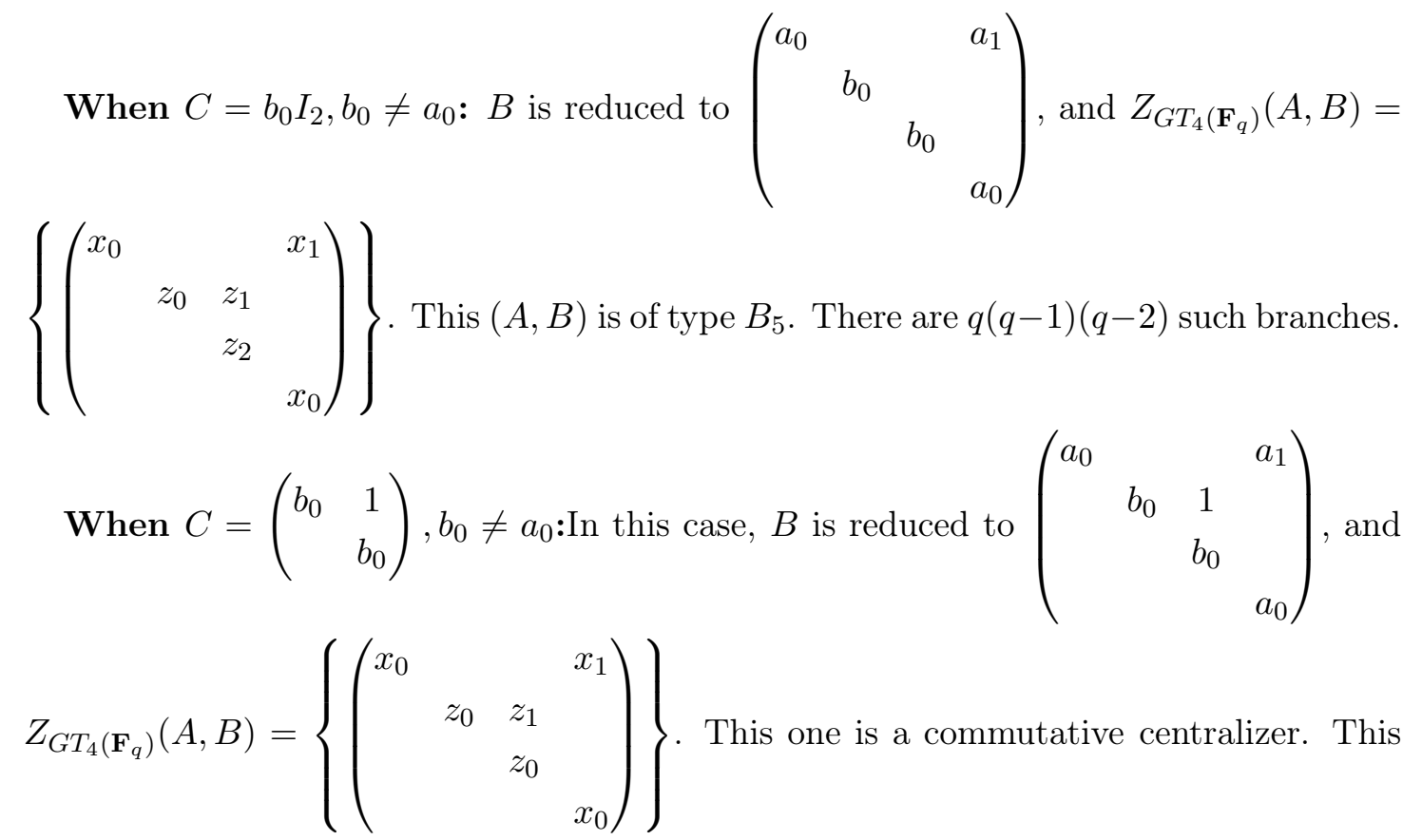
$(A, B)$ is of type $R_{3}$, and there are $q(q-1)(q-2)$ such branches.

When $C=\left(\begin{array}{ll}b_{0} & \\ & c_{0}\end{array}\right), b_{0}, c_{0} \neq a_{0}$, and $b_{0} \neq c_{0}$ :In this case, $B$ is reduced to $\left(\begin{array}{cccc}a_{0} & & & a_{1} \\ & b_{0} & & \\ & & c_{0} & \\ & & & a_{0}\end{array}\right)$, and $Z_{G T_{4}\left(\mathbf{F}_{q}\right)}(A, B)=\left\{\left(\begin{array}{cccc}x_{0} & & & x_{1} \\ & z_{0} & & \\ & z_{2} & \\ & & x_{0}\end{array}\right)\right\}$. This $(A, B)$ is of type $R$, and there are $q(q-1)(q-2)(q-3)$ such branches.

So, those are all the cases available.

Adding up all the branches of type $A_{8}$, we have a total of $q-1+q(q-1)+(q-1)^{2}=$ $2 q(q-1)$ branches.

Proposition 4.6. A matrix of type $A_{4}$ has:

\begin{tabular}{c|c||c|c}
\hline Branch & No. of Branches & Branch & No. of Branches \\
\hline$A_{4}$ & $q(q-1)$ & $t N T_{1}$ & $q(q-1)^{2}$ \\
$R_{1}$ & $q^{3}-q^{2}$ & $t N T_{5}$ & $q(q-1)$ \\
$R_{3}$ & $q^{2}(q-1)(q-2)$ & & \\
\hline
\end{tabular}

A new type $t N T_{5}$ appears with centralizer $\left\{\left(\begin{array}{cccc}a_{0} & a_{1} & b_{0} & b_{1} \\ & a_{0} & b_{0} \\ & & a_{0} & c_{1} \\ & & a_{0}\end{array}\right) \mid a_{0} \neq 0\right\}$. 
Proof. The canonical form of a matrix of this type is $A=\left(\begin{array}{cccc}a & 1 & & \\ & a & & \\ & a & 1 \\ & & a\end{array}\right)$. Then $Z_{G T_{4}\left(\mathbf{F}_{q}\right)}(A)=\left\{\left(\begin{array}{cccc}a_{0} & a_{1} & b_{0} & b_{1} \\ & a_{0} & & b_{0} \\ & & c_{0} & c_{1} \\ & & & c_{0}\end{array}\right) \mid a_{0}, c_{0} \neq 0\right\}$. Let $B=\left(\begin{array}{cccc}a_{0} & a_{1} & b_{0} & b_{1} \\ & a_{0} & & b_{0} \\ & & c_{0} & c_{1} \\ & & & c_{0}\end{array}\right)$, and $B^{\prime}=\left(\begin{array}{cccc}a_{0} & a_{1} & b_{0}^{\prime} & b_{1}^{\prime} \\ & a_{0} & & b_{0}^{\prime} \\ & & c_{0} & c_{1} \\ & & & c_{0}\end{array}\right)=X B X^{-1}$, where $X=\left(\begin{array}{cccc}x_{0} & x_{1} & y_{0} & y_{1} \\ & x_{0} & & y_{0} \\ & & z_{0} & z_{1} \\ & & & z_{0}\end{array}\right) . \quad X B=B^{\prime} X$ gives us the following:

$$
\begin{aligned}
x_{0} b_{0}+y_{0} c_{0} & =z_{0} b_{0}^{\prime}+y_{0} a_{0} \\
x_{0} b_{1}+x_{1} b_{0}+y_{0} c_{1}+y_{1} c_{0} & =y_{1} a_{0}+y_{0} a_{1}+z_{1} b_{0}^{\prime}+z_{0} b_{1}^{\prime}
\end{aligned}
$$

When $a_{0}=c_{0}$ : From Equation 4.17, we have $x_{0} b_{0}=z_{0} b_{0}^{\prime}$.

When $b_{0}=0$ : Equation 4.18 becomes $x_{0} b_{1}+y_{0} c_{1}=z_{0} b_{1}^{\prime}+y_{0} a_{1}$. Here we first look at what happens when $a_{1}=c_{1}$, and $b_{1}=0$. Here $B$ reduces to $\left(\begin{array}{cccc}a_{0} & a_{1} & & \\ & a_{0} & & \\ & a_{0} & a_{1} \\ & & a_{0}\end{array}\right)$, $Z_{G T_{4}\left(\mathbf{F}_{q}\right)}(A, B)=Z_{G T_{4}\left(\mathbf{F}_{q}\right)}(A) .(A, B)$ is of type $A_{4}$, and there are $q(q-1)$ such branches. When $a_{1}=c_{1}$, and $b_{1} \neq 0$. We can choose $x_{0}$ such that $b_{1}^{\prime}=1$. Thus $B$ is reduced to $\left(\begin{array}{cccc}a_{0} & a_{1} & & 1 \\ & a_{0} & & \\ & & a_{0} & a_{1} \\ & & & a_{0}\end{array}\right)$, and $Z_{G T_{4}\left(\mathbf{F}_{q}\right)}(A, B)=\left\{\left(\begin{array}{cccc}x_{0} & x_{1} & y_{0} & y_{1} \\ & x_{0} & & y_{0} \\ & & x_{0} & z_{1} \\ & & & x_{0}\end{array}\right)\right\}$. We see a centralizer, not isomorphic to the ones seen so far. Thus, we have a new type $t N T_{5} .(A, B)$ is of type $t N T_{5}$, and there are $q(q-1)$ such branches.

When $a_{1} \neq c_{1}$, in Equation 4.18, we can choose $y_{0}$, so that $b_{1}^{\prime}=0$. Thus, $B$ is reduced to $\left(\begin{array}{cccc}a_{0} & a_{1} & & \\ & a_{0} & & \\ & & a_{0} & c_{1} \\ & & & a_{0}\end{array}\right)$, and $Z_{G T_{4}\left(\mathbf{F}_{q}\right)}(A, B)=\left\{\left(\begin{array}{cccc}x_{0} & x_{1} & & y_{1} \\ & x_{0} & & \\ & & z_{0} & z_{1} \\ & & & z_{0}\end{array}\right)\right\}(A, B)$ is of type $t N T_{1}$, and there are $q(q-1)^{2}$ such branches.

When $b_{0} \neq 0$ : In Equation 4.17, choose $x_{0}$ such that $b_{0}^{\prime}=1$. Then, on replacing $b_{0}$ and $b_{0}^{\prime}$ by 1 in the same equation, we have $x_{0}=z_{0}$. Hence, Equation 4.18 becomes $x_{0} b_{1}+x_{1}+y_{0} c_{1}=x_{0} b_{1}^{\prime}+z_{1}+y_{0} a_{1}$. Hence, choose $z_{1}$ so that $b_{1}^{\prime}=0$. Then, $B$ is reduced 
to $\left(\begin{array}{cccc}a_{0} & a_{1} & 1 & \\ & a_{0} & & 1 \\ & & a_{0} & c_{1} \\ & & & a_{0}\end{array}\right)$, and $Z_{G T_{4}\left(\mathbf{F}_{q}\right)}(A, B)=\left\{\left(\begin{array}{ccc}x_{0} & x_{1} & \\ & x_{0} & \\ & & x_{0} \\ & & x_{1}+ \\ & & x_{0}\left(c_{1}-a_{1}\right)\end{array}\right)\right\}(A, B)$ is of type $R_{1}$, and there are $q^{2}(q-1)$ such branches.

When $a_{0} \neq c_{0}$ : In Equation 4.17, choose $y_{0}$ so that $b_{0}^{\prime}=0$. With this, Equation 4.18 becomes $x_{0} b_{1}+y_{1} c_{0}=z_{0} b_{1}^{\prime}+y_{1} a_{0}$. Choose $y_{1}$ such that $b_{1}^{\prime}=0$. Thus, $B$ is reduced to

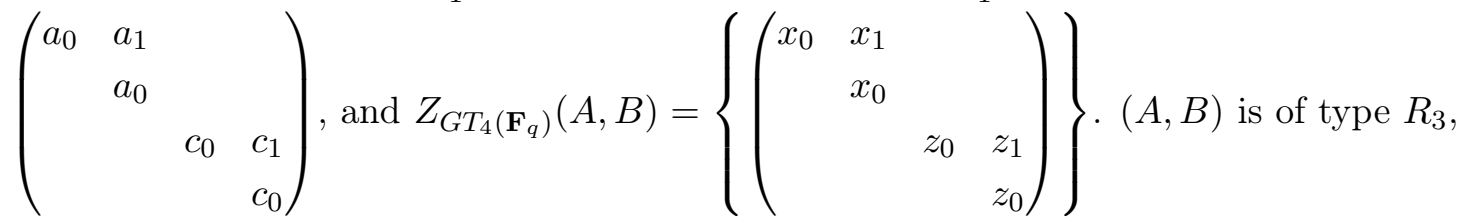
and there are $q^{2}(q-1)(q-2)=q^{4}-q^{3}$ such branches. Thus, there are no more cases left to deal with.

Proposition 4.7. An upper triangular matrix of type $A_{5}$ has $q^{2}(q-1)$ branches of type $A_{5}, q^{2}(q-1)(q-2)$ branches of type $R_{3}$, and $q^{2}\left(q^{2}-1\right)$ branches of type $N R_{1}$.

Proof. A matrix of type $A_{5}$ has the canonical form: $A=\left(\begin{array}{cccc}a & & & 1 \\ & a & 1 & \\ & a & \\ & & a\end{array}\right)$. Thus its centralizer $Z_{G T_{4}\left(\mathbf{F}_{q}\right)}(A)$ is: $\left\{\left(\begin{array}{cccc}a_{0} & & a_{2} & a_{3} \\ & b_{0} & b_{1} & b_{2} \\ & & b_{0} & \\ & & & a_{0}\end{array}\right)\right\}$. Let $B=\left(\begin{array}{cccc}a_{0} & & a_{2} & a_{3} \\ & b_{0} & b_{1} & b_{2} \\ & b_{0} & \\ & & & a_{0}\end{array}\right)$, and $B^{\prime}=\left(\begin{array}{cccc}a_{0} & & a_{2}^{\prime} & a_{3}^{\prime} \\ & b_{0}^{\prime} & b_{1}^{\prime} & b_{2} \\ & & b_{0} & \\ & & & a_{0}\end{array}\right)=X B X^{-1}$, where $X=\left(\begin{array}{cccc}x_{0} & & x_{2} & x_{3} \\ & y_{0} & y_{1} & y_{2} \\ & & y_{0} & \\ & & & x_{0}\end{array}\right)$. Thus, from $X B=$ $B^{\prime} X$, we have $a_{3}^{\prime}=a_{3}, b_{1}^{\prime}=b_{1}$, and the following equations:

$$
\begin{aligned}
x_{0} a_{2}+x_{2} b_{0} & =y_{0} a_{2}^{\prime}+x_{2} a_{0} \\
y_{0} b_{2}+a_{0} y_{2} & =x_{0} b_{2}^{\prime}+y_{2} b_{0}
\end{aligned}
$$

Case $a_{0}=b_{0}$. Equations 4.19 and 4.20 become $x_{0} a_{2}=y_{0} a_{2}^{\prime}$, and $y_{0} b_{2}=x_{0} b_{2}^{\prime}$ respectively. 
If $a_{2}=b_{2}=0$, the above equations are void, and we have $B$ reduced to $\left(\begin{array}{lllll}a_{0} & & & & a_{3} \\ & a_{0} & b_{1} & \\ & & a_{0} & \\ & & & a_{0}\end{array}\right)$,

and $Z_{G T_{4}\left(\mathbf{F}_{q}\right)}(A, B)=Z_{G T_{4}\left(\mathbf{F}_{q}\right)}(A)$. Thus $(A, B)$ is a branch of type $A_{5}$, and there are $q^{2}(q-1)$ such branches.

If $a_{2} \neq 0$, then choose $x_{0}$ so that $a_{2}^{\prime}=1$. Substituting $a_{2}$ with $a_{2}^{\prime}=1$ in the equation $x_{0} a_{2}=y_{0} a_{2}^{\prime}$, we get $x_{0}=y_{0}$, thus leaving us with $b_{2}^{\prime}=b_{2}$. Hence $B$ is reduced to $\left(\begin{array}{cccc}a_{0} & & 1 & a_{3} \\ & a_{0} & b_{1} & b_{2} \\ & & a_{0} & \\ & & & a_{0}\end{array}\right)$, and $Z_{G T_{4}\left(\mathbf{F}_{q}\right)}(A, B)=\left\{\left(\begin{array}{cccc}x_{0} & & x_{2} & x_{3} \\ & x_{0} & y_{1} & y_{2} \\ & & x_{0} & \\ & & & x_{0}\end{array}\right)\right\}$. Thus $(A, B)$ is a branch of type $N R_{1}$, and there are $q^{3}(q-1)$ such branches.

If $a_{2}=0$ and $b_{2} \neq 0$, then we choose $y_{0}$ such that $b_{2}^{\prime}=1$. Thus $B$ is reduced to $\left(\begin{array}{cccc}a_{0} & & & a_{3} \\ & a_{0} & b_{1} & 1 \\ & & a_{0} & \\ & & & a_{0}\end{array}\right)$, and $Z_{G T_{4}\left(\mathbf{F}_{q}\right)}(A, B)=\left\{\left(\begin{array}{cccc}x_{0} & & x_{2} & x_{3} \\ & x_{0} & y_{1} & y_{2} \\ & & x_{0} & \\ & & & x_{0}\end{array}\right)\right\}$. This branch too is of type $N R_{1}$, and there are $q^{2}(q-1)$ such branches.

If $a_{0} \neq b_{0}$. Then, in Equation 4.19, choose $x_{2}$ such that $a_{2}^{\prime}=0$. Similarly in Equation 4.20, choose $y_{2}$ such that $b_{2}^{\prime}=0$. Thus $B$ boils down to $\left(\begin{array}{llll}a_{0} & & & a_{3} \\ & b_{0} & b_{1} & \\ & b_{0} & \\ & & a_{0}\end{array}\right)$, and $Z_{G T_{4}\left(\mathbf{F}_{q}\right)}(A, B)=\left\{\left(\begin{array}{cccc}x_{0} & & & x_{3} \\ & y_{0} & y_{1} & \\ & & y_{0} & \\ & & & x_{0}\end{array}\right)\right\}$. This $(A, B)$ is of type $R_{3}$, and there are $q 2^{2}(q-1)(q-2)$ such branches.

Adding up the branches of type $N R_{1}$, we have a total of $q^{3}(q-1)+q^{2}(q-1)=q^{2}\left(q^{2}-1\right)$ branches of type $N R_{1}$.

Proposition 4.8. For a matrix of type $A_{6}$, the branchings are:

\begin{tabular}{c|c||c|c}
\hline Branch & No. of Branches & Branch & No. of Branches \\
\hline$A_{6}$ & $q(q-1)$ & $R_{3}$ & $q^{2}(q-1)(q-2)$ \\
$A_{5}$ & $q(q-1)^{2}$ & $t N T_{4}$ & $q^{2}(q-1)$ \\
$R_{1}$ & $q^{2}(q-1)$ & $N R_{1}$ & $q^{2}(q-1)$. \\
\hline \multicolumn{4}{r}{34}
\end{tabular}


Proof. A matrix of type $A_{6}$ has the canonical form $\left(\begin{array}{cccc}a & & 1 & \\ & a & & 1 \\ & a & \\ & & a\end{array}\right)$. The centralizer subgroup $Z_{G T_{4}\left(\mathbf{F}_{q}\right)}(A)$ is $\left\{\left(\begin{array}{ll}C & D \\ & C\end{array}\right) \mid C \in G T_{2}\left(\mathbf{F}_{q}\right)\right\}$, where $D=\left(\begin{array}{ll}d_{0} & d_{1} \\ d_{2} & d_{3}\end{array}\right)$, and $W=$ $\left(\begin{array}{ll}w_{0} & w_{1} \\ w_{2} & w_{3}\end{array}\right)$

Let $B=\left(\begin{array}{ll}C & D \\ & C\end{array}\right)$, and $B^{\prime}=\left(\begin{array}{ll}C^{\prime} & D^{\prime} \\ & C^{\prime}\end{array}\right)=X B X^{-1}$, where $X=\left(\begin{array}{cc}Z & W \\ & Z\end{array}\right)$. So $X B=B^{\prime} X$ leads to $Z C=C^{\prime} Z$. Hence, we can take $C$ to be a representative of a conjugacy class in $G T_{2}\left(\mathbf{F}_{q}\right)$, and $Z=Z_{G T_{2}\left(\mathbf{F}_{q}\right)}(C)$. We have the following equation

$$
Z D+W C=C W+D^{\prime} Z
$$

So the cases to deal with here are the three conjugacy class types in $G T_{2}\left(\mathbf{F}_{q}\right)$.

Case $C=\left(\begin{array}{cc}a_{0} & 1 \\ & a_{0}\end{array}\right)$ : here $Z=\left(\begin{array}{ll}x_{0} & x_{1} \\ & x_{0}\end{array}\right)$, and Equation 4.21 becomes:

$$
\left(\begin{array}{cc}
x_{0} d_{0}+x_{1} d_{2} & x_{0} d_{1}+x_{1} d_{3}+w_{0} \\
x_{0} d_{2} & x_{0} d_{3}+w_{2}
\end{array}\right)=\left(\begin{array}{cc}
w_{2}+x_{0} d_{0}^{\prime} & w_{3}+x_{1} d_{0}^{\prime}+x_{0} d_{1} \\
x_{0} d_{2}^{\prime} & x_{1} d_{2}^{\prime}+x_{0} d_{3}
\end{array}\right)
$$

Choose $w_{2}$ so that $d_{0}^{\prime}=0$. Thus, on replacing $d_{0}$ by 0 , we get $w_{2}=x_{1} d_{2}$, and hence $d_{3}^{\prime}=d_{3}$.

We can choose $w_{0}$ such that $d_{1}^{\prime}=0$. Thus $B$ is reduced to $\left(\begin{array}{cccc}a_{0} & 1 & & \\ & a_{0} & d_{2} & d_{3} \\ & a_{0} & 1 \\ & & a_{0}\end{array}\right)$, and $Z_{G T_{4}\left(\mathbf{F}_{q}\right)}(A, B)=\left\{\left(\begin{array}{cccc}x_{0} & x_{1} & w_{0} & w_{1} \\ & x_{0} & x_{1} d_{2} & w_{0}+x_{1} d_{3} \\ & & x_{0} & x_{1} \\ & & & x_{0}\end{array}\right)\right\}$. This $(A, B)$ is of type $R_{1}$, and there are $q^{2}(q-1)$ such branches.

Case $C=\left(\begin{array}{cc}a_{0} & \\ & b_{0}\end{array}\right), a_{0} \neq b_{0}$ : here $Z=\left(\begin{array}{ll}x_{0} & \\ & x_{3}\end{array}\right)$, and Equation 4.21 becomes:

$$
\left(\begin{array}{ll}
x_{0} d_{0}+a_{0} w_{0} & x_{0} d_{1}+w_{1} b_{0} \\
x_{3} d_{2}+a_{0} w_{2} & x_{3} d_{3}+b_{0} w_{3}
\end{array}\right)=\left(\begin{array}{ll}
a_{0} w_{0}+x_{0} d_{0}^{\prime} & w_{1} a_{0}+x_{3} d_{1} \\
b_{0} w_{2}+x_{0} d_{2} & b_{0} w_{3}+x_{3} d_{3}
\end{array}\right) .
$$


We have $d_{0}^{\prime}=d_{0}$ and $d_{3}^{\prime}=d_{3}$. As $a_{0} \neq b_{0}$, choose $w_{2}$ such that $d_{2}^{\prime}=0$, and $w_{1}$ so that $d_{1}^{\prime}=0$. Thus $B$ is reduced to $\left(\begin{array}{llll}a_{0} & & d_{0} & \\ & b_{0} & & d_{3} \\ & a_{0} & \\ & & d_{0}\end{array}\right)$, and $Z_{G T_{4}\left(\mathbf{F}_{q}\right)}(A, B)=$ $\left\{\left(\begin{array}{cccc}x_{0} & & w_{0} & \\ & x_{3} & & w_{3} \\ & & x_{0} & \\ & & & x_{3}\end{array}\right)\right\}$. This $(A, B)$ is of type $R_{3}$, and there are $q^{2}(q-1)(q-2)$ such branches.

Case $C=a_{0} I_{2}$ : Here Equation 4.21 becomes: $Z D=D^{\prime} Z$, where $Z \in T_{2}\left(\mathbf{F}_{q}\right)$. With $Z=\left(\begin{array}{ll}x_{0} & x_{1} \\ & x_{2}\end{array}\right)$, we see that:

$$
\left(\begin{array}{cc}
x_{0} d_{0}+x_{1} d_{2} & x_{0} d_{1}+x_{1} d_{3} \\
x_{3} d_{2} & x_{3} d_{3}
\end{array}\right)=\left(\begin{array}{cc}
x_{0} d_{0}^{\prime} & x_{1} d_{0}^{\prime}+x_{3} d_{1}^{\prime} \\
x_{0} d_{2}^{\prime} & x_{1} d_{2}^{\prime}+x_{3} d_{3}^{\prime}
\end{array}\right) .
$$

We see that $x_{0} d_{2}^{\prime}=x_{3} d_{2}$. We have two main cases here:

Case $d_{2}=0$. In this case, from Equation 4.22 we have $d_{0}^{\prime}=d_{0}$, and $d_{3}^{\prime}=d_{3}$, and we have $x_{0} d_{1}+\left(d_{3}-d_{0}\right) x_{1}=x_{3} d_{1}^{\prime}$.

When $d_{0}=d_{3}$, we have $x_{0} d_{1}=x_{3}^{\prime} d_{1}$. Now, if $d_{1}=0$. we have $B=\left(\begin{array}{ll}a_{0} I_{2} & d_{0} I_{2} \\ & a_{0} I_{2}\end{array}\right)$, and $Z_{G T_{4}\left(\mathbf{F}_{q}\right)}(A, B)=Z_{G T_{4}\left(\mathbf{F}_{q}\right)}(A)$. Thus, $(A, B)$ is of type $A_{6}$, and there are $q(q-1)$ such branches.

$$
\begin{aligned}
& \text { If } d_{1} \neq 0 \text {, choose } x_{0} \text { so that } d_{1}^{\prime}=1 \text {. Thus } B \text { is reduced to }\left(\begin{array}{cccc}
a_{0} & & d_{0} & 1 \\
& a_{0} & & d_{3} \\
& a_{0} & \\
& & a_{0}
\end{array}\right) \text {, and } \\
& \qquad Z_{G T_{4}\left(\mathbf{F}_{q}\right)}(A, B)=\left\{\left(\begin{array}{cccc}
x_{0} & x_{1} & w_{1} & w_{2} \\
& x_{0} & w_{2} & w_{3} \\
& & x_{0} & x_{1} \\
& & x_{0}
\end{array}\right)\right\} .
\end{aligned}
$$

$(A, B)$ is therefore of type $t N T_{4}$, and there are $q^{2}(q-1)$ such branches.

When $d_{0} \neq d_{3}$, in the $(1,2)$ th entry of Equation 4.22 , we choose $x_{1}$ so that $d_{1}^{\prime}=0$. Thus $B$ is reduced to $\left(\begin{array}{cccc}a_{0} & & d_{0} & \\ & a_{0} & & d_{3} \\ & & a_{0} & \\ & & & a_{0}\end{array}\right)$, and $Z_{G T_{4}\left(\mathbf{F}_{q}\right)}(A, B)=\left\{\left(\begin{array}{llll}x_{0} & & w_{1} & w_{2} \\ & x_{3} & w_{2} & w_{3} \\ & x_{0} & \\ & & & x_{3}\end{array}\right)\right\}$. 
This is isomorphic to the centralizer of a matrix of type $A_{5}$. Thus $(A, B)$ is a branch of type $A_{5}$, and there are $q^{2}(q-1)$ such branches.

Case $d_{2} \neq 0$. First, we choose $x_{0}$ such that $d_{2}^{\prime}=1$. On replacing $d_{2}$ with $d_{2}^{\prime}=1$ in Equation 4.22, and equating, we get $x_{0}=x_{3}$.

In the same equation, we can choose $x_{1}$ such that $d_{0}^{\prime}=0$. On replacing $d_{0}$ with $d_{0}^{\prime}=0$ and equating, we get $x_{1}=0$. Thus, $d_{3}^{\prime}=d_{3}$. Lastly, we have $x_{0} d_{1}=x_{0} d_{1}^{\prime}$, hence $d_{1}^{\prime}=d_{1}$.

Thus $B$ is reduced to $\left(\begin{array}{cccc}a_{0} & & & d_{1} \\ & a_{0} & 1 & d_{3} \\ & & a_{0} & \\ & & & a_{0}\end{array}\right)$, and $Z_{G T_{4}\left(\mathbf{F}_{q}\right)}(A, B)=\left\{\left(\begin{array}{cc}x_{0} I_{2} & W \\ & x_{0} I_{2}\end{array}\right) \mid W \in M_{2}\left(\mathbf{F}_{q}\right)\right\}$.

$(A, B)$ is a branch of type $N R_{1}$, and there are $q^{2}(q-1)$ such branches.

There are no other cases.

Proposition 4.9. The branching rules of remaining A types are as follows.

(1) For a matrix of type $A_{7}$, there are $q^{2}(q-1)$ branches of type $A_{7}, q^{2}(q-1)$ branches of type $R_{1}$, and $q^{2}(q-1)(q-2)$ branches of type $R_{2}$.

(2) The type $A_{8}$ has $q^{2}(q-1)$ branches of type $A_{8}, q^{3}-q$ branches of type $R_{1}$, and $q^{2}(q-1)(q-2)$ branches of type $R_{2}$.

(3) The type $A_{9}$ has $q^{2}(q-1)$ branches of type $A_{9},\left(q^{2}-q\right)\left(q^{2}-1\right)$ branches of type $R_{1}$.

Proof. $\quad$ (1) A matrix of type $A_{7}$ has two non-similar canonical forms, $\left(\begin{array}{cccc}a & 1 & & \\ & a & 1 & \\ & & a & \\ & & & a\end{array}\right)$, and $\left(\begin{array}{cccc}a & & & \\ & a & 1 & \\ & & a & 1 \\ & & & a\end{array}\right)$. As their centralizer subgroups in $T_{4}\left(\mathbf{F}_{q}\right)$ are conjugate in $G L_{4}\left(\mathbf{F}_{q}\right)$, we may prove the branching for any one. Let $A=\left(\begin{array}{llll}a & 1 & & \\ & a & 1 & \\ & & a & \\ & & & a\end{array}\right)$. Then $Z_{G T_{4}\left(\mathbf{F}_{q}\right)}(A)=\left\{\left(\begin{array}{cccc}a_{0} & a_{1} & a_{2} & a_{3} \\ & a_{0} & a_{1} & \\ & & a_{0} & \\ & & & d_{0}\end{array}\right) \mid a_{0}, d_{0} \neq 0\right\}$. 


$$
\begin{gathered}
\text { Let } B=\left(\begin{array}{cccc}
a_{0} & a_{1} & a_{2} & a_{3} \\
& a_{0} & a_{1} & \\
& & a_{0} & \\
& & & d_{0}
\end{array}\right) \text {, and } B^{\prime}=\left(\begin{array}{cccc}
a_{0} & a_{1}^{\prime} & a_{2}^{\prime} & a_{3}^{\prime} \\
& a_{0} & a_{1}^{\prime} & \\
& & a_{0} & \\
& & & d_{0}
\end{array}\right)=X B X^{-1} \text {, where } \\
X=\left(\begin{array}{cccc}
x_{0} & x_{1} & x_{2} & x_{3} \\
& x_{0} & x_{1} & \\
& & x_{0} & \\
& & z_{0}
\end{array}\right) \text {. From } X B=B^{\prime} X \text { we have } a_{1}^{\prime}=a_{1}, a_{2}^{\prime}=a_{2} \text {, and this }
\end{gathered}
$$

equation:

$$
x_{0} a_{3}+x_{3} d_{0}=z_{0} a_{3}^{\prime}+x_{3} a_{0}
$$

If $a_{0}=d_{0}$, then Equation 4.23 becomes $x_{0} a_{3}=z_{0} z_{3}^{\prime}$.

Here, if $a_{3}=0$, then $B$ is reduced to $\left(\begin{array}{cccc}a_{0} & a_{1} & a_{2} & \\ & a_{0} & a_{1} & \\ & & a_{0} & \\ & & & a_{0}\end{array}\right)$, and $Z_{G T_{4}\left(\mathbf{F}_{q}\right)}(A, B)=$ $Z_{G T_{4}\left(\mathbf{F}_{q}\right)}(A)$. Thus $(A, B)$ is of type $A_{7}$, and there are $q^{2}(q-1)$ such branches,. If $a_{3} \neq 0$, then choose $z_{0}$ so that $a_{3}^{\prime}=1$. Thus, $B$ is reduced to $\left(\begin{array}{cccc}a_{0} & a_{1} & a_{2} & 1 \\ & a_{0} & a_{1} & \\ & & a_{0} & \\ & & & a_{0}\end{array}\right)$, and $Z_{G T_{4}\left(\mathbf{F}_{q}\right)}(A, B)=\left\{\left(\begin{array}{cccc}x_{0} & x_{1} & x_{2} & x_{3} \\ & x_{0} & x_{1} & \\ & & x_{0} & \\ & & & x_{0}\end{array}\right)\right\}$. This $(A, B)$ is of type $R_{1}$, and there are $q^{2}(q-1)$ such branches.

When $a_{0} \neq d_{0}$, then, in Equation 4.23 , choose $x_{3}$ so that $a_{3}^{\prime}=0$. Thus $B$ is reduced to $\left(\begin{array}{cccc}a_{0} & a_{1} & a_{2} & \\ & a_{0} & a_{1} & \\ & & a_{0} & \\ & & & d_{0}\end{array}\right)$, and $Z_{G T_{4}\left(\mathbf{F}_{q}\right)}(A, B)=\left\{\left(\begin{array}{cccc}x_{0} & x_{1} & x_{2} & \\ & x_{0} & x_{1} & \\ & & x_{0} & \\ & & & z_{0}\end{array}\right)\right\}$. This too is commutative (by a routine check). $(A, B)$ is of type $R$, and there are $q^{2}(q-1)(q-2)$ such branches. There are no other cases left to analyze, so these are all the branches. 
(2) Matrices of type $A_{8}$ have either of the two non-similar canonical forms: $\left(\begin{array}{cccc}a & 1 & & \\ & a & & 1 \\ & & a & \\ & & & a\end{array}\right)$, and $\left(\begin{array}{cccc}a & & 1 & \\ & a & & \\ & & a & 1 \\ & & & a\end{array}\right)$. As their centralizers are conjugate in $G L_{4}\left(\mathbf{F}_{q}\right)$, it is enough

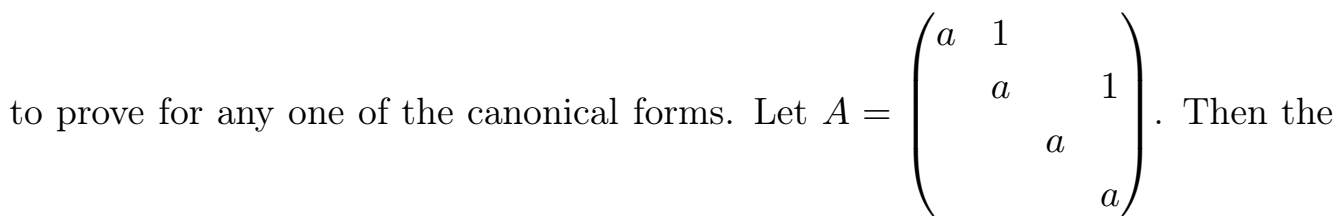
centralizer of $A$ is $Z_{G T_{4}\left(\mathbf{F}_{q}\right)}(A)=\left\{\left(\begin{array}{cccc}a_{0} & a_{1} & b & a_{2} \\ & a_{0} & & a_{1} \\ & & d & c \\ & & & a_{0}\end{array}\right)\right\}$. let $B \in Z_{G T_{4}\left(\mathbf{F}_{q}\right)}(A)$ be the matrix $\left(\begin{array}{cccc}a_{0} & a_{1} & b & a_{2} \\ & a_{0} & & a_{1} \\ & & d & c \\ & & & a_{0}\end{array}\right)$, and let $B^{\prime}=\left(\begin{array}{cccc}a_{0} & a_{1}^{\prime} & b^{\prime} & a_{2}^{\prime} \\ & a_{0} & & a_{1}^{\prime} \\ & & d & c^{\prime} \\ & & & a_{0}\end{array}\right)=X B X^{-1}$, where $X=\left(\begin{array}{cccc}x_{0} & x_{1} & y & x_{2} \\ & x_{0} & & x_{1} \\ & & z & w \\ & & & x_{0}\end{array}\right)$. Now $X B=X B^{\prime} X$ leads us to $a_{1}^{\prime}=a_{1}$, and the following equations:

$$
\begin{aligned}
x_{0} b+y d & =z b^{\prime}+y a_{0} \\
z c+w a_{0} & =x_{0} c^{\prime}+w d \\
x_{0} a_{2}+y c & =w b^{\prime}+x_{0} a_{2}^{\prime}
\end{aligned}
$$

When $a_{0}=d$ : Here, Equations 4.24 and 4.25 become $x_{0} b=z b^{\prime}$, and $z c=x_{0} c^{\prime}$ respectively.

When $b=c=0$, Equation 4.26 becomes $x_{0} a_{2}=x_{0} a_{2}^{\prime}$, hence $a_{2}^{\prime}=a_{2} . B$ is reduced to $\left(\begin{array}{cccc}a_{0} & a_{1} & & a_{2} \\ & a_{0} & & a_{1} \\ & & a_{0} & \\ & & & a_{0}\end{array}\right)$, and $Z_{G T_{4}\left(\mathbf{F}_{q}\right)}(A, B)=Z_{G T_{4}\left(\mathbf{F}_{q}\right)}(A) . \quad(A, B)$ is of type $A_{8}$, and there are $q^{2}(q-1)$ such branches. 
When $b \neq 0$, choose $z$ such that $b^{\prime}=1$. Then, on substituting $b$ with $b^{\prime}=1$ in Equation 4.24, we get $z=x_{0}$. Thus, we have $c=c^{\prime}$. And, in Equation 4.26, choose $w$ so that $a_{2}^{\prime}=0$. Thus $B$ is reduced to $\left(\begin{array}{cccc}a_{0} & a_{1} & 1 & \\ & a_{0} & & a_{1} \\ & a_{0} & \\ & & a_{0}\end{array}\right)$, and $Z_{G T_{4}\left(\mathbf{F}_{q}\right)}(A, B)=\left\{\left(\begin{array}{cccc}x_{0} & x_{1} & y & x_{2} \\ & x_{0} & & x_{1} \\ & & x_{0} & c y \\ & & & x_{0}\end{array}\right)\right\} .(A, B)$ is of type $R_{1}$, and there are $q^{2}(q-1)$ such branches.

When $b=0$ and $c \neq 0$, in Equation 4.25, choose $x_{0}$ such that $c^{\prime}=1$. Then Equation 4.26 becomes $x_{0} a_{2}+y=x_{0} a_{2}^{\prime}$. Thus, choose $y$ so that $a_{2}^{\prime}=0$. Hence $B$ is reduced to $\left(\begin{array}{cccc}a_{0} & a_{1} & & \\ & a_{0} & & a_{1} \\ & & a_{0} & 1 \\ & & & a_{0}\end{array}\right)$, and $Z_{G T_{4}\left(\mathbf{F}_{q}\right)}(A, B)=\left\{\left(\begin{array}{cccc}x_{0} & x_{1} & & x_{2} \\ & x_{0} & & x_{1} \\ & & x_{0} & w \\ & & x_{0}\end{array}\right)\right\}$. $(A, B)$ is of type $R_{1}$, and there are $q(q-1)$ such branches.

There are no further cases for us to look at here. We now look at the case of $a_{0} \neq d$.

When $a_{0} \neq d$ : In Equation 4.24, choose $y$ such that $b^{\prime}=0$, and in Equation 4.25, choose $w$ such that $c^{\prime}=0$. Then Equation 4.26 becomes $x_{0} a_{2}=$ $a_{0} a_{2}^{\prime}$, implying $a_{2}^{\prime}=a_{2}$. $B$ reduces to $\left(\begin{array}{llll}a_{0} & a_{1} & & a_{2} \\ & a_{0} & & a_{1} \\ & & d & \\ & & & a_{0}\end{array}\right)$, and $Z_{G T_{4}\left(\mathbf{F}_{q}\right)}(A, B)=$ $\left\{\left(\begin{array}{cccc}x_{0} & x_{1} & & x_{2} \\ & x_{0} & & x_{1} \\ & & z & \\ & & & x_{0}\end{array}\right)\right\}$. This too is a commutative centralizer. $(A, B)$ is of type $R_{2}$, and there are $q^{2}(q-1)(q-2)$ such branches. Now, there are no more cases to look at. Adding up all the branches of type $R_{1}$, we have a total of $q^{2}(q-1)+q(q-1)=q^{3}-q$ branches of type $R_{1}$. 
(3) A matrix of type $A_{9}$ has the following canonical form: $A=\left(\begin{array}{cccc}a & 1 & 1 & \\ & a & & \\ & a & 1 \\ & & & a\end{array}\right)$. Then we have $Z_{G T_{4}\left(\mathbf{F}_{q}\right)}(A)=\left\{\left(\begin{array}{cccc}a_{0} & a_{1} & b & a_{2} \\ & a_{0} & & c \\ & & a_{0} & b-c \\ & & & a_{0}\end{array}\right)\right\}$. Let $B=\left(\begin{array}{cccc}a_{0} & a_{1} & b & a_{2} \\ & a_{0} & c \\ & & a_{0} & b-c \\ & & & a_{0}\end{array}\right)$, and $B^{\prime}=\left(\begin{array}{cccc}a_{0} & a_{1}^{\prime} & b^{\prime \prime} & a_{2} \\ & a_{0} & & c^{\prime} \\ & & a_{0} & b^{\prime}-c^{\prime}\end{array}\right)=X B X^{-1}$, where $X=\left(\begin{array}{cccc}x_{0} & x_{1} & y & x_{2} \\ & x_{0} & & w \\ & & x_{0} & y-w \\ & & & x_{0}\end{array}\right)$,

with $x_{0} \neq 0$. So, $X B=B^{\prime} X$ leaves us with $a_{1}^{\prime}=a_{1}, b^{\prime}=b$, and $c^{\prime}=c$, and the following equation:

$$
x_{0} a_{2}+\left(x_{1}-x_{2}\right) c=x_{0} a_{2}^{\prime}+\left(a_{1}-b\right) w
$$

When $a_{1}=b$ and $c=0$ Here Equation 4.27 ends up as $a_{2}^{\prime}=a_{2} . \quad B$ is thus reduced to $\left(\begin{array}{cccc}a_{0} & a_{1} & a_{1} & a_{2} \\ & a_{0} & & \\ & & a_{0} & a_{1} \\ & & & a_{0}\end{array}\right)$, and $Z_{G T_{4}\left(\mathbf{F}_{q}\right)}(A, B)=Z_{G T_{4}\left(\mathbf{F}_{q}\right)}(A)$. Thus $(A, B)$ is of type $A_{9}$, and there are $q^{2}(q-1)$ such branches.

When $a_{1} \neq b$ : Here, in Equation 4.27, we choose $w$ such that $a_{2}^{\prime}=0$. $B$ is thus reduced to $\left(\begin{array}{cccc}a_{0} & a_{1} & b & \\ & a_{0} & & c \\ & & a_{0} & b-c \\ & & & a_{0}\end{array}\right)$, with $Z_{G T_{4}\left(\mathbf{F}_{q}\right)}(A, B)=\left\{\left(\begin{array}{cccc}x_{0} & x_{1} & y & x_{2} \\ & x_{0} & & \frac{\left(x_{1}-y\right)}{a_{1}-b} c \\ & & x_{0} & y-\frac{\left(x_{1}-y\right)}{a_{1}-b} c \\ & & & x_{0}\end{array}\right)\right\}$.

$(A, B)$ is therefore of type $R_{1}$, and there are $q^{2}(q-1)^{2}$ such branches.

When $a_{1}=b$, and $c \neq 0$ : In Equation 4.27, choose $x_{1}$ or $y$ such that $a_{2}^{\prime}=0$.

Thus, $B$ is reduced to $\left(\begin{array}{cccc}a_{0} & a_{1} & a_{1} & \\ & a_{0} & & c \\ & & a_{0} & a_{1}-c \\ & & & a_{0}\end{array}\right)$. So $Z_{G T_{4}\left(\mathbf{F}_{q}\right)}(A, B)=\left\{\left(\begin{array}{cccc}x_{0} & x_{1} & x_{1} & x_{2} \\ & x_{0} & & w \\ & & x_{0} & x_{1}-w \\ & & & x_{0}\end{array}\right)\right\}$.

This $(A, B)$ too is of type $R_{1}$, and there are $q(q-1)^{2}$ such branches.

With this, we have no other cases to look at. Thus, we have $q^{3}$ branches of type $A_{9}$, and $q(q-1)^{2}+q^{2}(q-1)^{2}=\left(q^{2}-q\right)\left(q^{2}-1\right)$ branches of type $R_{1}$. 
4.2. Branching rules for type $B$. Matrices of types $B 1, B 2, B 3, B 4, B 5$ are in block form of the kind $A=\left(\begin{array}{cc}C_{1} & \\ & C_{2}\end{array}\right)$, where $C_{1} \in G T_{m_{1}}\left(\mathbf{F}_{q}\right)$, and $C_{2} \in G T_{m_{2}}\left(\mathbf{F}_{q}\right)$, where $m_{1}+m_{2}=4$. Thus, $Z_{G T_{4}\left(\mathbf{F}_{q}\right)}(A)=\left\{\left(\begin{array}{cc}X_{1} & \\ & X_{2}\end{array}\right)\right\}$ where $X_{1} \in Z_{G T_{m_{1}}}\left(C_{1}\right)$ and $X_{2} \in$ $Z_{G T_{m_{2}}}\left(C_{2}\right)$. Thus, the branches of $A$ are of the form $\left(\begin{array}{ll}D_{1} & \\ & D_{2}\end{array}\right)$, where $D_{1}$ is a branch of $C_{1}$, and $D_{2}$ is a branch of $C_{2}$. With this argument, we can prove the following proposition.

Proposition 4.10. The branching rules are as follows:

(1) For a matrix of type $B_{1}$, there are:

\begin{tabular}{c|c||c|c}
\hline Branch & No. of Branches & Branch & No. of Branches \\
\hline$B_{1}$ & $(q-1)^{2}$ & $R_{3}$ & $(q-1)^{2}$ \\
$B_{5}$ & $2(q-1)^{2}$ & $R_{4}$ & $2(q-1)^{2}(q-2)$ \\
$B_{6}$ & $2(q-1)^{2}(q-2)$ & $R_{5}$ & $(q-1)^{2}(q-2)^{2}$ \\
\hline
\end{tabular}

(2) For a matrix of type $B_{2}$, there are:

\begin{tabular}{c|c||c|c}
\hline Branch & No. of Branches & Branch & No. of Branches \\
\hline$B_{2}$ & $(q-1)^{2}$ & $R_{2}$ & $(q-1)^{2}$ \\
$B_{3}$ & $(q-1)^{2}$ & $R_{4}$ & $(q-1)^{2}(q-2)$ \\
$B_{4}$ & $(q-1)^{2}$ & $R_{5}$ & $(q-1)^{2}(q-2)(q-3)$ \\
$B_{6}$ & $(q-1)^{2}(q-2)$ & & \\
\hline
\end{tabular}

(3) For a matrix of type $B_{3}$, there are $q(q-1)^{2}$ branches of type $B_{3}, q(q-1)^{2}$ branches of type $R_{2}$, and $q(q-1)^{2}(q-2)$ branches of type $R_{4}$.

(4) For a matrix of type $B_{4}$, there are, $q(q-1)^{2}$ branches of type $B_{4},\left(q^{2}-1\right)(q-1)$ branches of type $R_{2}$, and $q(q-1)^{2}(q-2)$ branches of type $R_{4}$.

(5) For a matrix of type $B_{5}$, there are $q(q-1)^{2}$ branches of type $B_{5}, q(q-1)^{2}$ branches of type $R_{3}$, and $q(q-1)^{2}(q-2)$ branches of type $R_{4}$.

Finally,

Proposition 4.11. For a matrix of type $B_{6}$, there are, $(q-1)^{3}$ branches of type $B_{6}$, $(q-1)^{3}$ branches of type $R_{4}$, and $(q-1)^{3}(q-2)$ branches of type $R_{5}$.

Proof. A matrix of type $B_{6}$ has the canonical form: $A=\left(\begin{array}{ccc}a & & \\ & a & \\ & & b\end{array}\right)$. Here, $Z_{G T_{4}\left(\mathbf{F}_{q}\right)}(A)=$ $\left\{\left(\begin{array}{lll}C & & \\ & c_{0} & \\ & & d_{0}\end{array}\right) \mid C \in G T_{2}\left(\mathbf{F}_{q}\right), c_{0}, d_{0} \neq 0\right\}$. Enumerating the conjugacy classes of $G T_{2}\left(\mathbf{F}_{q}\right)$ gives us the branches mentioned.

4.3. Branching Rules of the New Types. While determining the branching rules of the existing types of conjugacy classes of $G T_{4}\left(\mathbf{F}_{q}\right)$, we came across six new types of 
simultaneous conjugacy classes of pairs of commuting matrices. We called them $t N T_{1}$, $t N T_{2}, t N T_{3}, t N T_{4}, t N T_{5}$, and $N R_{1}$. In this subsection, we shall focus on the branching rules of these new types.

Proposition 4.12. A commuting tuple of type $t N T_{1}$ has $q^{2}(q-1)$ branches of type $t N T_{1}$, $q^{2}(q-1)$ branches of type $R_{1}$, and $q^{2}(q-1)(q-2)$ branches of type $R_{3}$.

Proof. For a commuting pair $(A, B)$ of matrices of type $t N T_{1}$, the centralizer is $Z_{G T_{4}\left(\mathbf{F}_{q}\right)}(A, B)=$ $\left\{\left(\begin{array}{cccc}a_{0} & a_{1} & & a_{3} \\ & a_{0} & & \\ & & c_{0} & c_{1} \\ & & & c_{0}\end{array}\right) \mid a_{0}, c_{0} \neq 0\right\}$. Let $C=\left(\begin{array}{cccc}a_{0} & a_{1} & & a_{3} \\ & a_{0} & & \\ & & c_{0} & c_{1} \\ & & & c_{0}\end{array}\right)$, and $C^{\prime}=\left(\begin{array}{cccc}a_{0} & a_{1}^{\prime} & & a_{3}^{\prime} \\ & a_{0} & & \\ & c_{0} & c_{1}^{\prime} \\ & & & c_{0}\end{array}\right)=$ $X C X^{-1}$ by $X=\left(\begin{array}{cccc}x_{0} & x_{1} & & x_{3} \\ & x_{0} & & \\ & & z_{0} & z_{1} \\ & & & z_{0}\end{array}\right) . X C=C^{\prime} X$ leads us to $a_{1}^{\prime}=a_{1}, c_{1}^{\prime}=c_{1}$, and just one equation:

$$
x_{0} a_{3}+x_{3} b_{0}=z_{0} a_{3}^{\prime}+x_{3} a_{0} .
$$

When $a_{0}=c_{0}$ : Here Equation 4.28 becomes $x_{0} a_{3}=z_{0} a_{3}^{\prime}$.

So, we have two cases over here: $a_{3}=0$, and $a_{3} \neq 0$.

When $a_{3}=0, C$ is reduced to $\left(\begin{array}{cccc}a_{0} & a_{1} & & \\ & a_{0} & & \\ & & a_{0} & c_{1} \\ & & & a_{0}\end{array}\right)$, with $Z_{G T_{4}\left(\mathbf{F}_{q}\right)}(A, B, C)=Z_{G T_{4}\left(\mathbf{F}_{q}\right)}(A, B)$. $(A, B, C)$ is of type $t N T_{1}$, and there are $q^{2}(q-1)$ such branches.

When $a_{3} \neq 0$, we choose $z_{0}$ such that $a_{3}^{\prime}=1$. Here, $C$ is reduced to $\left(\begin{array}{llll}a_{0} & a_{1} & & 1 \\ & a_{0} & & \\ & & a_{0} & c_{1} \\ & & & a_{0}\end{array}\right)$, with $Z_{G T_{4}\left(\mathbf{F}_{q}\right)}(A, B, C)=\left\{\left(\begin{array}{cccc}x_{0} & x_{1} & & x_{3} \\ & x_{0} & & \\ & & x_{0} & z_{1} \\ & & & x_{0}\end{array}\right)\right\}$. This $(A, B, C)$ is of type $R_{1}$, and there are $q^{2}(q-1)$ such branches.

So now, with $a_{0}=c_{0}$, we have no other cases left to analyse. We move on to the case of $a_{0} \neq c_{0}$. 
When $a_{0} \neq c_{0}$ : Here, in Equation 4.28, we can choose $x_{3}$ so that $a_{3}^{\prime}=0$. So $C$ is reduced

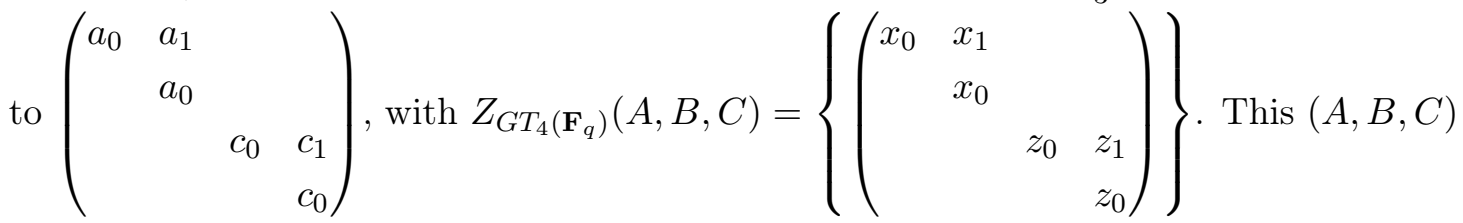
is of type $R_{3}$, and there are $q^{2}(q-1)(q-2)$ such branches.

So, with this, we have no other cases to look at.

Proposition 4.13. The new type $t N T_{2}$ has $q^{2}(q-1)$ branches of type $t N T_{2}, q^{2}(q-1)(q-$ 2) branches of type $R_{2}$, and $q^{2}\left(q^{2}-1\right)$ branches of type $N R_{1}$.

Proof. For a commuting pair $(A, B)$ of type $t N T_{2}$, the centralizer is

$$
\begin{aligned}
& Z_{G T_{4}\left(\mathbf{F}_{q}\right)}(A, B)=\left\{\left(\begin{array}{cccc}
a_{0} & & b_{0} & b_{1} \\
& a_{0} & b_{2} & b_{3} \\
& & a_{0} & \\
& & & c_{0}
\end{array}\right) \begin{array}{c}
a_{0}, b_{0}, b_{1} \\
b_{2}, b_{3}, c_{0} \in \mathbf{F}_{q}
\end{array}\right\} \text {. Let } C=\left(\begin{array}{cccc}
a_{0} & & b_{0} & b_{1} \\
& a_{0} & b_{2} & b_{3} \\
& a_{0} & \\
& & c_{0}
\end{array}\right), \\
& \text { and } C^{\prime}=\left(\begin{array}{cccc}
a_{0} & & b_{0}^{\prime} & b_{1}^{\prime} \\
& a_{0} & b_{2}^{\prime} & b_{3}^{\prime} \\
& & a_{0} & \\
& & & c_{0}
\end{array}\right)=X C X^{-1} \text { for some } X=\left(\begin{array}{cccc}
x_{0} & & y_{0} & y_{1} \\
& x_{0} & y_{2} & y_{3} \\
& x_{0} & \\
& & z_{0}
\end{array}\right) \text {. So, equating }
\end{aligned}
$$

$X C=C^{\prime} X$ leads us to $b_{0}^{\prime}=b_{0}, b_{2}^{\prime}=b_{2}$, and the following equations:

$$
\begin{aligned}
x_{0} b_{1}+y_{1} c_{0} & =z_{0} b_{1}^{\prime}+y_{1} a_{0} \\
x_{0} b_{3}+y_{3} c_{0} & =z_{0} b_{3}^{\prime}+y_{3} a_{0}
\end{aligned}
$$

We have two main cases: $a_{0}=c_{0}$, and $a_{0} \neq c_{0}$ :

When $a_{0}=c_{0}$ : Here, Equation 4.29 becomes $x_{0} b_{1}=z_{0} b_{1}^{\prime}$, and Equation 4.30 becomes $x_{0} b_{3}=z_{0} b_{3}^{\prime}$.

When $b_{1}=b_{3}=0, C$ is reduced to $\left(\begin{array}{llll}a_{0} & & b_{0} & \\ & a_{0} & b_{2} & \\ & & a_{0} & \\ & & a_{0}\end{array}\right)$, with $Z_{G T_{4}\left(\mathbf{F}_{q}\right)}(A, B, C)=$ $Z_{G T_{4}\left(\mathbf{F}_{q}\right)}(A, B)$. Thus $(A, B, C)$ is of type $t N T_{2}$, and there are $q^{2}(q-1)$ such branches.

When $b_{1} \neq 0$. In Equation 4.29, choose $z_{0}$ such that $b_{1}^{\prime}=1$. Then, on replacing $b_{1}$ and $b_{1}^{\prime}$ by 1 in the same equation, we get $z_{0}=x_{0}$. Hence, Equation 4.30 becomes $x_{0} b_{3}=$ 
$x_{0} b_{3}^{\prime}$, hence $b_{3}^{\prime}=b_{3}$. $C$ is reduced to $\left(\begin{array}{llll}a_{0} & & b_{0} & 1 \\ & a_{0} & b_{2} & b_{3} \\ & a_{0} & \\ & & a_{0}\end{array}\right)$, with $Z_{G T_{4}\left(\mathbf{F}_{q}\right)}(A, B, C)=$ $\left\{\left(\begin{array}{llll}x_{0} & & y_{0} & y_{1} \\ & x_{0} & y_{2} & y_{3} \\ & & x_{0} & \\ & & & x_{0}\end{array}\right)\right\} .(A, B, C)$ is of type $N R_{1}$. There are $q^{3}(q-1)$ such branches. When $b_{1}=0$, and $b_{3} \neq 0$. In Equation 4.30, choose $z_{0}$ so that $b_{3}^{\prime}=1$. Thus $C$ is reduced to $\left(\begin{array}{cccc}a_{0} & & b_{0} & \\ & a_{0} & b_{2} & 1 \\ & & a_{0} & \\ & & & a_{0}\end{array}\right)$, with $Z_{G T_{4}\left(\mathbf{F}_{q}\right)}(A, B, C)=\left\{\left(\begin{array}{llll}x_{0} & & y_{0} & y_{1} \\ & x_{0} & y_{2} & y_{3} \\ & x_{0} & \\ & & x_{0}\end{array}\right)\right\}$. $(A, B, C)$ is of type $N R_{1}$. There are $q^{2}(q-1)$ such branches. We have exhausted all the cases under $a_{0}=c_{0}$.

When $a_{0} \neq c_{0}$ : Here, in Equation 4.29, choose $y_{1}$ so that $b_{1}^{\prime}=0$, and in Equation 4.30, choose $y_{3}$ so that $b_{3}^{\prime}=0$. C is thus reduced to $\left(\begin{array}{llll}a_{0} & & b_{0} & \\ & a_{0} & b_{2} & \\ & a_{0} & \\ & & b_{0}\end{array}\right)$, with $Z_{G T_{4}\left(\mathbf{F}_{q}\right)}(A, B, C)=\left\{\left(\begin{array}{cccc}x_{0} & & y_{0} & \\ & x_{0} & y_{2} & \\ & & x_{0} & \\ & & & z_{0}\end{array}\right)\right\}$. This $(A, B, C)$ is of type $R_{2}$, and there are $q^{2}(q-1)(q-2)$ such branches.

This leaves us with no further cases to analyse. Adding up the branches of type $N R_{1}$, we have a total of $q^{2}(q-1)+q^{3}(q-1)=q^{2}\left(q^{2}-1\right)$ branches of type $N R_{1}$.

Proposition 4.14. A commuting pair of type $t N T_{3}$ has $q^{2}(q-1)$ branches of type $t N T_{3}$, $q^{2}(q-1)$ branches of type $R_{1}, q^{2}(q-1)(q-2)$ branches of type $R_{2}$, and $q\left(q^{2}-1\right)$ branches of type $N R_{1}$.

Proof. Let $(A, B)$ be a pair of commuting matrices of type $t N T_{3}$. Their common centralizer is $Z_{G T_{4}\left(\mathbf{F}_{q}\right)}(A, B)=\left\{\left(\begin{array}{cc}D & E \\ & D_{11} I_{2}\end{array}\right) \mid D \in T_{2}\left(\mathbf{F}_{q}\right), E \in M_{2}\left(\mathbf{F}_{q}\right)\right\}$. Let $C=\left(\begin{array}{cc}D & E \\ & a_{0} I_{2}\end{array}\right)$, where $D=\left(\begin{array}{cc}a_{0} & a_{1} \\ & b_{0}\end{array}\right)$ and $E=\left(\begin{array}{ll}b_{0} & b_{1} \\ b_{2} & b_{3}\end{array}\right)$. Let $C^{\prime}=\left(\begin{array}{cc}D^{\prime} & E^{\prime} \\ & a_{0} I_{2}\end{array}\right)=X C X^{-1}$, where $X=$ $\left(\begin{array}{cc}Z & Y \\ & x_{0} I_{2}\end{array}\right) \in Z_{G T_{4}\left(\mathbf{F}_{q}\right)}(A, B)$, where $Z=\left(\begin{array}{cc}x_{0} & x_{1} \\ & z_{0}\end{array}\right) \in G T_{2}\left(\mathbf{F}_{q}\right)$, and $Y=\left(\begin{array}{ll}y_{0} & y_{1} \\ y_{2} & y_{3}\end{array}\right)$. 
So $X C=C^{\prime} X$ leaves us with the following $Z D=D^{\prime} Z$. Thus $D$ can be taken to be a representative of a conjugacy class in $G T_{2}\left(\mathbf{F}_{q}\right)$, and $Z \in Z_{G T_{2}\left(\mathbf{F}_{q}\right)}(D)$. We are therefore left with the following equation:

$$
Z E+a_{0} Y=D Y+x_{0} E^{\prime}
$$

Exapanding this, we have:

$$
\left(\begin{array}{cc}
x_{0} b_{0}+x_{1} b_{2} & x_{0} b_{1}+x_{1} b_{3} \\
z_{0} b_{2} & z_{0} b_{3}
\end{array}\right)+\left(\begin{array}{cc}
-a_{1} y_{2} & -a_{1} y_{3} \\
\left(a_{0}-b_{0}\right) y_{2} & \left(a_{0}-b_{0}\right) y_{3}
\end{array}\right)=\left(\begin{array}{ll}
x_{0} b_{0}^{\prime} & x_{0} b_{1}^{\prime} \\
x_{0} b_{2}^{\prime} & x_{0} b_{3}^{\prime}
\end{array}\right)
$$

When $D=a_{0} I_{2}$ : Here Equation 4.31 becomes:

$$
\left(\begin{array}{cc}
x_{0} b_{0}+x_{1} b_{2} & x_{0} b_{1}+x_{1} b_{2} \\
z_{0} b_{2} & z_{0} b_{3}
\end{array}\right)+=\left(\begin{array}{cc}
x_{0} b_{0}^{\prime} & x_{0} b_{1}^{\prime} \\
x_{0} b_{2}^{\prime} & x_{0} b_{3}^{\prime}
\end{array}\right)
$$

When $b_{2}=b_{3}=0$, we have $b_{0}^{\prime}=b_{0}$, and $b_{1}^{\prime}=b_{1}$. Thus, $C$ is reduced to $\left(\begin{array}{cccc}a_{0} & & b_{0} & b_{1} \\ & a_{0} & & \\ & a_{0} & \\ & & a_{0}\end{array}\right)$, and $Z_{G T_{4}\left(\mathbf{F}_{q}\right)}(A, B, C)=Z_{G T_{4}\left(\mathbf{F}_{q}\right)}(A, B) . \quad(A, B, C)$ is of type $t N T_{3}$, and there are $q^{2}(q-1)$ such branches.

When $b_{2} \neq 0$, choose $z_{0}$ such that $b_{2}^{\prime}=1$. Thus, on replacing $b_{0}$ by $b_{0}^{\prime}=1$ in Equation 4.31, we get $z_{0}=x_{0}$. Hence $b_{3}^{\prime}=b_{3}$. With these, Eqaution 4.31 becomes

$$
\left(\begin{array}{cc}
x_{0} b_{0}+x_{1} & x_{0} b_{1}+x_{1} b_{2} \\
1 & b_{3}
\end{array}\right)+=\left(\begin{array}{cc}
x_{0} b_{0}^{\prime} & x_{0} b_{1}^{\prime} \\
1 & b_{3}^{\prime}
\end{array}\right)
$$

Choose $x_{1}$ so that $b_{0}^{\prime}=0$. On replacing $b_{0}$ by $b_{0}^{\prime}=0$ in the above equation, we have $x_{1}=0$. Thus $b_{1}^{\prime}=b_{1}$. So $C$ is reduced to $\left(\begin{array}{cccc}a_{0} & & & b_{1} \\ & a_{0} & 1 & b_{3} \\ & & a_{0} & \\ & & a_{0}\end{array}\right)$ with $Z_{G T_{4}\left(\mathbf{F}_{q}\right)}(A, B, C)=$ $\left\{\left(\begin{array}{llll}x_{0} & & y_{0} & y_{1} \\ & x_{0} & y_{2} & y_{3} \\ & & x_{0} & \\ & & & x_{0}\end{array}\right)\right\} .(A, B, C)$ is of type $N R_{1}$, and there are $q^{2}(q-1)$ such branches.

When $b_{2}=0$ and $b_{3} \neq 0$. Choose $z_{0}$ so that $b_{3}^{\prime}=1$. Equation 4.31 becomes

$$
\left(\begin{array}{cc}
x_{0} b_{0} & x_{0} b_{1}+x_{1} \\
0 & 1
\end{array}\right)_{46}+=\left(\begin{array}{cc}
x_{0} b_{0}^{\prime} & x_{0} b_{1}^{\prime} \\
0 & 1
\end{array}\right)
$$


Hence, $b_{0}^{\prime}=b_{0}$, and choose $x_{1}$ so that $b_{1}^{\prime}=0 . C$ is reduced to $\left(\begin{array}{cccc}a_{0} & & b_{0} & \\ & a_{0} & & 1 \\ & a_{0} & \\ & & a_{0}\end{array}\right)$, with $Z_{G T_{4}\left(\mathbf{F}_{q}\right)}(A, B, C)=\left\{\left(\begin{array}{cccc}x_{0} & & y_{0} & y_{1} \\ & x_{0} & y_{2} & y_{3} \\ & & x_{0} & \\ & & & x_{0}\end{array}\right)\right\}$. This $(A, B, C)$ too is of type $N R_{1}$, and there are $q(q-1)$ such branches.

With this, we have no other cases to analyse when $D=a_{0} I_{2}$. When $D=\left(\begin{array}{cc}a_{0} & 1 \\ & a_{0}\end{array}\right)$ : Here $Z=\left(\begin{array}{cc}x_{0} & x_{1} \\ & x_{0}\end{array}\right)$. Equation 4.31 becomes:

$$
\left(\begin{array}{cc}
x_{0} b_{0}+x_{1} b_{2} & x_{0} b_{1}+x_{1} b_{3} \\
x_{0} b_{2} & x_{0} b_{3}
\end{array}\right)+\left(\begin{array}{cc}
-y_{2} & -y_{3} \\
0 & 0
\end{array}\right)=\left(\begin{array}{cc}
x_{0} b_{0}^{\prime} & x_{0} b_{1}^{\prime} \\
x_{0} b_{2}^{\prime} & x_{0} b_{3}^{\prime}
\end{array}\right)
$$

We have from this $b_{2}^{\prime}=b_{2}, b_{3}^{\prime}=b_{3}$, and we can choose $y_{2}$ so that $b_{0}^{\prime}=0$ and $y_{3}$ such that $b_{1}^{\prime}=0$. Hence $C$ is reduced to $\left(\begin{array}{cccc}a_{0} & 1 & & \\ & a_{0} & b_{2} & b_{3} \\ & & a_{0} & \\ & & a_{0}\end{array}\right)$, with $Z_{G T_{4}\left(\mathbf{F}_{q}\right)}(A, B, C)=$ $\left\{\left(\begin{array}{cccc}x_{0} & x_{1} & x_{2} & x_{3} \\ & x_{0} & b_{2} x_{1} & b_{3} x_{1} \\ & & x_{0} & \\ & & & x_{0}\end{array}\right)\right\}$. This $(A, B, C)$ is of type $R_{1}$, and there are $q^{2}(q-1)$ such branches.

When $C=\left(\begin{array}{cc}a_{0} & \\ & c_{0}\end{array}\right), c_{0} \neq a_{0}$ : Here $Z=\left(\begin{array}{cc}x_{0} & \\ & z_{0}\end{array}\right)$. Equation 4.31 becomes:

$$
\left(\begin{array}{ll}
x_{0} b_{0} & x_{0} b_{1} \\
z_{0} b_{2} & z_{0} b_{3}
\end{array}\right)+\left(\begin{array}{cc}
\left(a_{0}-c_{0}\right) y_{2} & \left(a_{0}-c_{0}\right) y_{3}
\end{array}\right)=\left(\begin{array}{ll}
x_{0} b_{0}^{\prime} & x_{0} b_{1}^{\prime} \\
x_{0} b_{2}^{\prime} & x_{0} b_{3}^{\prime}
\end{array}\right)
$$

We have $b_{0}^{\prime}=b_{0}$ and $b_{1}^{\prime}=b_{1}$. Choose $y_{2}$ and $y_{3}$ such that $b_{2}^{\prime}=b_{3}^{\prime}=0$. $C$ is reduced to $\left(\begin{array}{cccc}a_{0} & & b_{0} & b_{1} \\ & c_{0} & & \\ & & a_{0} & \\ & & & a_{0}\end{array}\right)$, and $\left\{\left(\begin{array}{cccc}x_{0} & & y_{0} & y_{1} \\ & z_{0} & & \\ & & x_{0} & \\ & & & x_{0}\end{array}\right)\right\}$. Here $(A, B, C)$ is of type $R_{2}$, and there are $q^{2}(q-1)(q-2)$ such branches.

With this, we have no other cases to deal with.

Adding up the branches of type $N R_{1}$, we have a total of $q(q-1)+q^{2}(q-1)=q\left(q^{2}-1\right)$ branches of this type. 
Proposition 4.15. For a pair of commuting matrices of type $t N T_{4}$, there are $q^{2}(q-1)$ branches of type $t N T_{4}, q^{2}(q-1)^{2}$ branches of type $R_{1}$, and $q\left(q^{2}-1\right)(q-1)$ branches of type $N R_{1}$.

Proof. The centralizer of a commuting pair $(A, B)$ of this type is

$$
Z_{G T_{4}\left(\mathbf{F}_{q}\right)}(A, B)=\left\{\left(\begin{array}{ccc}
a_{0} & a_{1} & \\
& a_{0} & B_{1} \\
& a_{0} & a_{1} \\
& & a_{0}
\end{array}\right) \mid a_{0} \neq 0, B_{1} \in M_{2}(F q)\right\} .
$$

This was seen, and proved in [Sh1, Lemma 5.14] as the new type $N T_{1}$.

Proposition 4.16. For a commuting pair of type $t N T_{5}$, there are $q^{2}(q-1)$ branches of type $t N T_{5}$, and $q\left(q^{2}-1\right)(q-1)$ branches of type $R_{1}$.

Proof. The centralizer of a commuting pair $(A, B)$ of type $t N T_{5}$ is:

$$
Z_{G T_{4}\left(\mathbf{F}_{q}\right)}(A, B)=\left\{\left(\begin{array}{cccc}
a_{0} & a_{1} & b_{0} & b_{1} \\
& a_{0} & & b_{0} \\
& & a_{0} & c_{1} \\
& & & a_{0}
\end{array}\right) \mid a_{0} \neq 0\right\}
$$

Let $C=\left(\begin{array}{cccc}a_{0} & a_{1} & b_{0} & b_{1} \\ & a_{0} & & b_{0} \\ & & a_{0} & c_{1} \\ & & & a_{0}\end{array}\right)$, and $C^{\prime}=\left(\begin{array}{cccc}a_{0} & a_{1} & b_{0}^{\prime} & b_{1}^{\prime} \\ & a_{0} & & b_{0}^{\prime} \\ & & a_{0} & c_{1} \\ & & & a_{0}\end{array}\right)=X C X^{-1}$, for some $X=$

$$
\left(\begin{array}{cccc}
x_{0} & x_{1} & y_{0} & y_{1} \\
& x_{0} & & y_{0} \\
& & x_{0} & z_{1} \\
& & & x_{0}
\end{array}\right) \text {. So } X C=C^{\prime} X \text { leads us to } b_{0}^{\prime}=b_{0}, \text { and the equation: }
$$

$$
x_{0} b_{1}+x_{1} b_{0}+y_{0} c_{1}=x_{0} b_{1}^{\prime}+z_{1} b_{0}+y_{0} a_{1} .
$$

We have two main cases: $a_{1}=c_{1}$ and $a_{1} \neq c_{1}$.

When $a_{1}=c_{1}$ : Equation 4.32 becomes $x_{0} b_{1}+x_{1} b_{0}=x_{0} b_{1}^{\prime}+z_{1} b_{0}$.

When $b_{0}=0$, we have $b_{1}^{\prime}=b_{1}$. $C$ is reduced to $\left(\begin{array}{cccc}a_{0} & a_{1} & & b_{1} \\ & a_{0} & & \\ & & a_{0} & a_{1} \\ & & a_{0}\end{array}\right)$, with $Z_{G T_{4}\left(\mathbf{F}_{q}\right)}(A, B, C)=$ $Z_{G T_{4}\left(\mathbf{F}_{q}\right)}(A, B) .(A, B, C)$ is thus of type $t N T_{5}$, and there are $q^{2}(q-1)$ such branches. 
When $b_{0} \neq 0$, choose $z_{1}$ such that $b_{1}^{\prime}=0 . C$ is reduced to $\left(\begin{array}{cccc}a_{0} & a_{1} & b_{0} & \\ & a_{0} & & b_{0} \\ & a_{0} & a_{1} \\ & & a_{0}\end{array}\right)$, with $Z_{G T_{4}\left(\mathbf{F}_{q}\right)}(A, B, C)=\left\{\left(\begin{array}{cccc}x_{0} & x_{1} & y_{0} & y_{1} \\ & x_{0} & & y_{0} \\ & & x_{0} & x_{1} \\ & & & x_{0}\end{array}\right)\right\} .(A, B, C)$ is of type $R_{1}$, and there are $q(q-1)^{2}$ such branches.

So, we have no other cases to look at for $a_{1}=c_{1}$.

$a_{1} \neq c_{1}$ : In Equation 4.32 , choose $y_{0}$ so that $b_{1}^{\prime}=0$. Thus, $C$ is reduced to $\left(\begin{array}{cccc}a_{0} & a_{1} & b_{0} & \\ & a_{0} & & b_{0} \\ & & a_{0} & c_{1} \\ & & & a_{0}\end{array}\right)$, with $Z_{G T_{4}\left(\mathbf{F}_{q}\right)}(A, B, C)=\left\{\left(\begin{array}{cccc}x_{0} & x_{1} & \frac{b_{0}\left(z_{1}-x_{1}\right)}{c_{1}-a_{1}} & \\ & x_{0} & & \frac{b_{0}\left(z_{1}-x_{1}\right)}{c_{1}-a_{1}} \\ & & x_{0} & z_{1} \\ & & & x_{0}\end{array}\right)\right\}$. Here $(A, B, C)$ is of type $R_{1}$, and there are $q^{2}(q-1)^{2}$ such branches.

With this, we have no other case to look at. So, adding up the branches of type $R$, we have a total of $q(q-1)^{2}+q^{2}(q-1)^{2}=q\left(q^{2}-1\right)(q-1)$ branches of type $R_{1}$.

Proposition 4.17. For a commuting pair of type $N R_{1}$, there are $q^{4}(q-1)$ branches of type $N R_{1}$.

Proof. The centralizer of a commuting pair $(A, B)$ of type $N R_{1}$ is

$$
Z_{G T_{4}\left(\mathbf{F}_{q}\right)}(A, B)=\left\{\left(\begin{array}{cc}
a_{0} I_{2} & D \\
& a_{0} I_{2}
\end{array}\right) \mid a_{0} \neq 0, D \in M_{2}\left(\mathbf{F}_{q}\right)\right\} .
$$

The result follows, as this is a commutative subgroup.

\section{BRANCHING IN $U T_{3}(q)$}

For the unitriangular group $U T_{3}\left(\mathbf{F}_{q}\right)$, the conjugacy classes are as follows: 


\begin{tabular}{|c|c|c|c|}
\hline Canonical Form & No. of Classes & Centralizer & Name of Type \\
\hline $\begin{array}{c}\left(\begin{array}{lll}1 & 0 & a \\
0 & 1 & 0 \\
0 & 0 & 1\end{array}\right) \\
a \in \mathbf{F}_{q}\end{array}$ & $q$ & $U T_{3}\left(\mathbf{F}_{q}\right)$ & $C$ \\
\hline $\begin{array}{c}\left(\begin{array}{lll}1 & a & 0 \\
0 & 1 & 0 \\
0 & 0 & 1\end{array}\right) \\
a \in \mathbf{F}_{q}^{*}\end{array}$ & $(q-1)$ & $\left\{\left(\begin{array}{ccc}1 & x_{0} & x_{1} \\
& 1 & \end{array}\right) \mid x_{0}, x_{1} \in \mathbf{F}_{q}\right\}$ & $R_{1}$ \\
\hline $\begin{array}{l}\left(\begin{array}{lll}1 & 0 & 0 \\
0 & 1 & a \\
0 & 0 & 1\end{array}\right) \\
a \in \mathbf{F}_{q}^{*}\end{array}$ & $(q-1)$ & 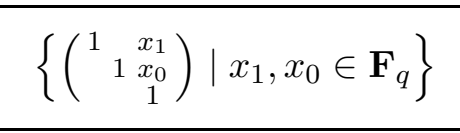 & $R_{1}$ \\
\hline $\begin{array}{l}\left(\begin{array}{lll}1 & a & 0 \\
0 & 1 & b \\
0 & 0 & 1\end{array}\right) \\
a, b \in \mathbf{F}_{q}^{*}\end{array}$ & $(q-1)^{2}$ & 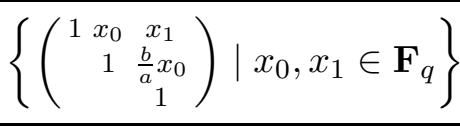 & $R_{2}$ \\
\hline
\end{tabular}

We see that there are two types here: central $C$ and regular $R$. Note that the centralizers of both regulars $R_{1}$ and $R_{2}$ are isomorphic (not conjugate). For the type $C$, the centralizer is full group $U T_{3}\left(\mathbf{F}_{q}\right)$, thus all types appear in the first column. For the regular type, it has $q^{2}$ branches of the same $R$ type, as the centralizer is commutative, of size $q^{2}$, hence the number of branches is $q^{2}$.

Theorem 5.1. The branching matrix (with the order of type $C, R 1$ ):

$$
B_{U T_{3}\left(\mathbf{F}_{q}\right)}=\left(\begin{array}{cc}
q & 0 \\
q^{2}-1 & q^{2}
\end{array}\right) .
$$

We prove the branching rules below.

Proposition 5.2. An upper unitriangular matrix of type $C$ has $q$ branches of type $C$, and $q^{2}-1$ branches of the type $R$.

Proof. The result follows as matrices of this type are central.

Proposition 5.3. A matrix of of any of the $R$ types has $q^{2}$ branches of the same $R$ type.

Proof. A matrix of any of the $R$ types is a Regular type, hence its centralizer in $U T_{3}\left(\mathbf{F}_{q}\right)$ is commutative, of size $q^{2}$, hence the number of branches is $q^{2}$.

\section{BRANChING IN $U T_{4}(q)$}

We shift our focus to commuting tuples of matrices in $U T_{4}\left(\mathbf{F}_{q}\right)$. The conjugacy classes according to the types of this group are listed in Appendix B 
Theorem 6.1. The branching rules for the upper unitriangular group is given by the following matrix (with order $C, A_{1}, A_{2}, A_{3}, R_{1}, R_{2}$ ):

$$
B_{U T_{4}\left(\mathbf{F}_{q}\right)}=\left(\begin{array}{cccccc}
q & 0 & 0 & 0 & 0 & 0 \\
2(q-1) & q^{2} & 0 & 0 & 0 & 0 \\
(q-1)^{2} & 0 & q^{2} & 0 & 0 & 0 \\
q\left(q^{2}-1\right) & 0 & 0 & q^{2} & 0 & 0 \\
q(q-1) & q\left(q^{2}-1\right) & q^{2}(q-1) & q\left(q^{2}-1\right) & q^{4} & 0 \\
\left(q^{2}-1\right)(q-1) & q^{2}(q-1) & q\left(q^{2}-1\right) & 0 & 0 & q^{3}
\end{array}\right) .
$$

The first column corresponds to type $C$, thus all types of $U T_{4}\left(\mathbf{F}_{q}\right)$ appears. The last two columns are the regular types. There are no new types here. The proof for other columns is listed below in propositions.

Proposition 6.2. An upper unitriangular matrix of type $A_{1}$ has $q^{2}$ branches of type $A_{1}$, and $q\left(q^{2}-1\right)$ branches of type $R_{1}$, and $q^{2}(q-1)$ branches of type $R_{2}$.

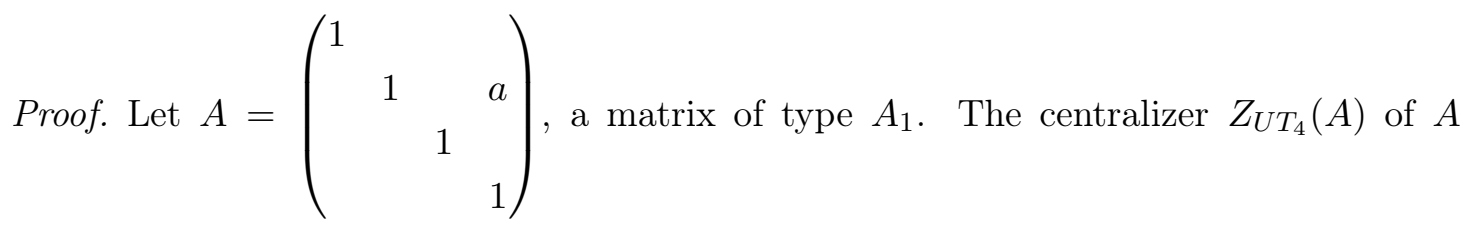

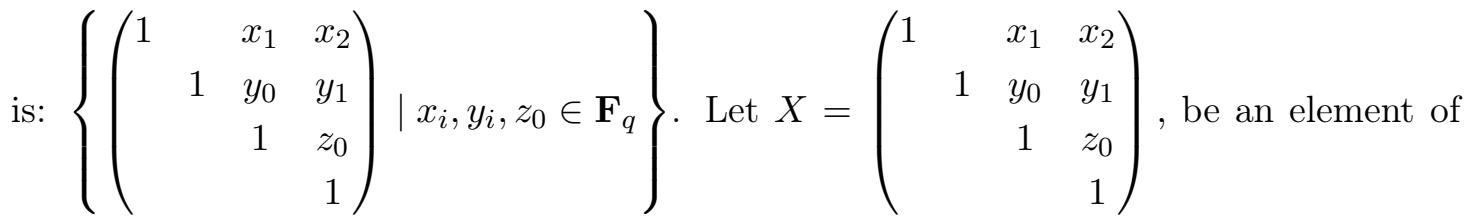
$Z_{U T_{4}}(A)$. Let $B=\left(\begin{array}{cccc}1 & & b_{1} & b_{2} \\ & 1 & c_{0} & c_{1} \\ & & 1 & d_{0} \\ & & & 1\end{array}\right)$, and $B^{\prime}=\left(\begin{array}{cccc}1 & & b_{1}^{\prime} & b_{2}^{\prime} \\ & 1 & c_{0}^{\prime} & c_{1}^{\prime} \\ & 1 & d_{0}^{\prime} \\ & & & 1\end{array}\right)$ be the conjugate of $B$ by $X$, i.e., $B^{\prime}=X B X^{-1}$. Thus equating $X B=B^{\prime} X$ leads us to $b_{0}^{\prime}=b_{0}, c_{0}^{\prime}=c_{0}$, $c_{1}^{\prime}=c_{1}$, and the following equations:

$$
\begin{aligned}
& x_{0} c_{0}+b_{1}=y_{0} b_{0}^{\prime}+b_{1}^{\prime} \\
& x_{0} c_{1}+b_{2}=y_{1} b_{0}^{\prime}+b_{2}^{\prime}
\end{aligned}
$$

We use these to simplify $B$ to the branches mentioned in the statement of the proposition.

Proposition 6.3. An upper unitriangular matrix of type $A_{2}$ has $q^{2}$ branches of type $A_{2}$, and $q^{2}(q-1)$ branches of type $R_{1}$, and $q\left(q^{2}-1\right)$ branches of $R_{2}$. 
Proof. Given $A=\left(\begin{array}{cccc}1 & & a & \\ & 1 & & b \\ & & 1 & \\ & & & 1\end{array}\right)$, where $a, b \in \mathbf{F}_{q}^{*}$. the canonical form of a matrix of type $A_{2}$. The centralizer of $A, Z_{U T_{4}}(A)$ is $\left\{\left(\begin{array}{cccc}1 & x_{0} & x_{1} & x_{2} \\ & 1 & y_{0} & y_{1} \\ & & 1 & \lambda x_{0} \\ & & & 1\end{array}\right) \mid \lambda=\frac{b}{a}, x_{i}, y_{i}, z_{0} \in \mathbf{F}_{q}\right\}$. Let $X=\left(\begin{array}{cccc}1 & x_{0} & x_{1} & x_{2} \\ & 1 & y_{0} & y_{1} \\ & & 1 & \lambda x_{0} \\ & & & 1\end{array}\right)$ be an element of $Z_{U T_{4}}(A)$. Let $B=\left(\begin{array}{cccc}1 & b_{0} & b_{1} & b_{2} \\ & 1 & c_{0} & c_{1} \\ & 1 & \lambda b_{0} \\ & & & 1\end{array}\right)$, and $B^{\prime}=\left(\begin{array}{cccc}1 & b_{0}^{\prime} & b_{1}^{\prime} & b_{2}^{\prime} \\ & 1 & c_{0}^{\prime} & c_{1}^{\prime} \\ & & 1 & \lambda b_{0}^{\prime} \\ & & & 1\end{array}\right)$ be the conjugate of $B$ by $X$. Thus equating $X B=B^{\prime} X$ gives us the following equations:

$$
\begin{gathered}
b_{0}=b_{0}^{\prime} \\
c_{0}=c_{0}^{\prime} \\
x_{0} c_{0}+b_{1}=y_{0} b_{0}^{\prime}+b_{1}^{\prime} \\
\lambda b_{0} y_{0}+c_{1}=\lambda x_{0} c_{0}^{\prime}+c_{1}^{\prime} \\
x_{0} c_{1}+\lambda b_{0} x_{1}+b_{2}=y_{1} b_{0}^{\prime}+\lambda b_{1}^{\prime} x_{0} b_{2}^{\prime}
\end{gathered}
$$

Using these we reduce $B$ to the mentioned branches.

Proposition 6.4. An upper triangular matrix of type $A_{3}$ has $q^{2}$ branches of type $A_{3}$, and $q\left(q^{2}-1\right)$ branches of type $R_{1}$.

Proof. One of the canonical forms of an upper triangular matrix of type $A_{3}$ is $A=$ $\left(\begin{array}{cccc}1 & a & & \\ & 1 & & \\ & & 1 & \\ & & & 1\end{array}\right)$, where $a \in \mathbf{F}_{q}^{*}$. Here $Z_{U T_{4}\left(\mathbf{F}_{q}\right)}(A)=\left\{\left(\begin{array}{cccc}1 & x_{0} & x_{1} & x_{2} \\ & 1 & & \\ & & 1 & z_{0} \\ & & & 1\end{array}\right) \mid x_{i}, z_{0} \in \mathbf{F}_{q}\right\}$.

Let $X=\left(\begin{array}{cccc}1 & x_{0} & x_{1} & x_{2} \\ & 1 & & \\ & & 1 & z_{0} \\ & & & 1\end{array}\right)$, be an element of $Z_{U T_{4}}(A)$. Let $B=\left(\begin{array}{cccc}1 & b_{0} & b_{1} & b_{2} \\ & 1 & & \\ & & 1 & d_{0} \\ & & & 1\end{array}\right)$, and 
$B^{\prime}=\left(\begin{array}{cccc}1 & b_{0}^{\prime} & b_{1}^{\prime} & b_{2}^{\prime} \\ & 1 & & \\ & & 1 & d_{0}^{\prime} \\ & & & 1\end{array}\right)$ be the conjugate of $B$ by $X$, i.e., $B^{\prime}=X B X^{-1}$. Thus equating $X B=B^{\prime} X$ leads us to the $b_{0}^{\prime}=b_{0}, b_{1}^{\prime}=b_{1}, d_{0}^{\prime}=d_{0}$, and the following equation:

$$
x_{1} d_{0}+b_{2}=z_{0} b_{1}^{\prime}+b_{2}^{\prime}
$$

We use these to simplify $B$ to the branches mentioned in the statement of the proposition.

Proposition 6.5. A matrix of the $R_{1}$ type has $q^{4}$ branches of type $R_{1}$ and $A$ matrix of the $R_{2}$ type has $q^{3}$ branches of type $R_{2}$.

Proof. The type $R_{1}$ and $R_{2}$ are Regular types, hence the centralizer of matrices of such a type is a commutative.

Proof of Theorem 6.1. From the data in Propositions 6.2 to 6.5, we summarize the branching rules for $U T_{4}$, as in the table described in the theorem.

Here are some isomorphisms between centralizers of matrices of the same $z$-class for some $z$-classes in $U T_{4}\left(\mathbf{F}_{q}\right)$.

Proposition 6.6. The centralizer of conjugacy classes with representative $\left(\begin{array}{ccccc}1 & & a & \\ & 1 & & \\ & & 1 & \\ & & & 1\end{array}\right)$ and $\left(\begin{array}{cccc}1 & & & \\ & 1 & & a \\ & & 1 & \\ & & & 1\end{array}\right)$, for $a \in \mathbf{F}_{q}^{*}$ are isomorphic.

Proof. The centralizer of conjugacy class with representative $\left(\begin{array}{cccc}1 & & a & \\ & 1 & & \\ & & 1 & \\ & & & 1\end{array}\right)$ is

$$
\left\{\left(\begin{array}{cccc}
1 & x_{0} & x_{1} & x_{2} \\
& 1 & y_{0} & y_{1} \\
& & 1 & \\
& & & 1
\end{array}\right) \mid x_{i}, y_{i} \in \mathbf{F}_{q}\right\} .
$$


The centralizer of conjugacy class with representative $\left(\begin{array}{cccc}1 & & & \\ & 1 & & a \\ & & 1 & \\ & & & 1\end{array}\right)$ is $\left\{\left(\begin{array}{cccc}1 & & x_{1} & x_{2} \\ & 1 & y_{0} & y_{1} \\ & & 1 & z_{0} \\ & & & 1\end{array}\right) \mid x_{i}, y_{i}, z_{0} \in \mathbf{F}_{q}\right\}$.

The following map gives isomorphism between these two centralizers.

$$
\left(\begin{array}{cccc}
1 & & x_{1} & x_{2} \\
& 1 & y_{0} & y_{1} \\
& & 1 & z_{0} \\
& & & 1
\end{array}\right) \mapsto\left(\begin{array}{cccc}
1 & -z_{0} & y_{1}-z_{0} y_{0} & x_{2}-x_{1} z_{0} \\
& 1 & y_{0} & x_{1} \\
& & 1 & \\
& & & 1
\end{array}\right)
$$

Proposition 6.7. The centralizers of all conjugacy classes of type $A_{3}$ are isomorphic.

Proof. There are six conjugacy classes of type $A_{3}$. In the following table, we give the centralizer of these conjugacy classes. We also set a notation for these conjugcay classes which will be used later in this proof.

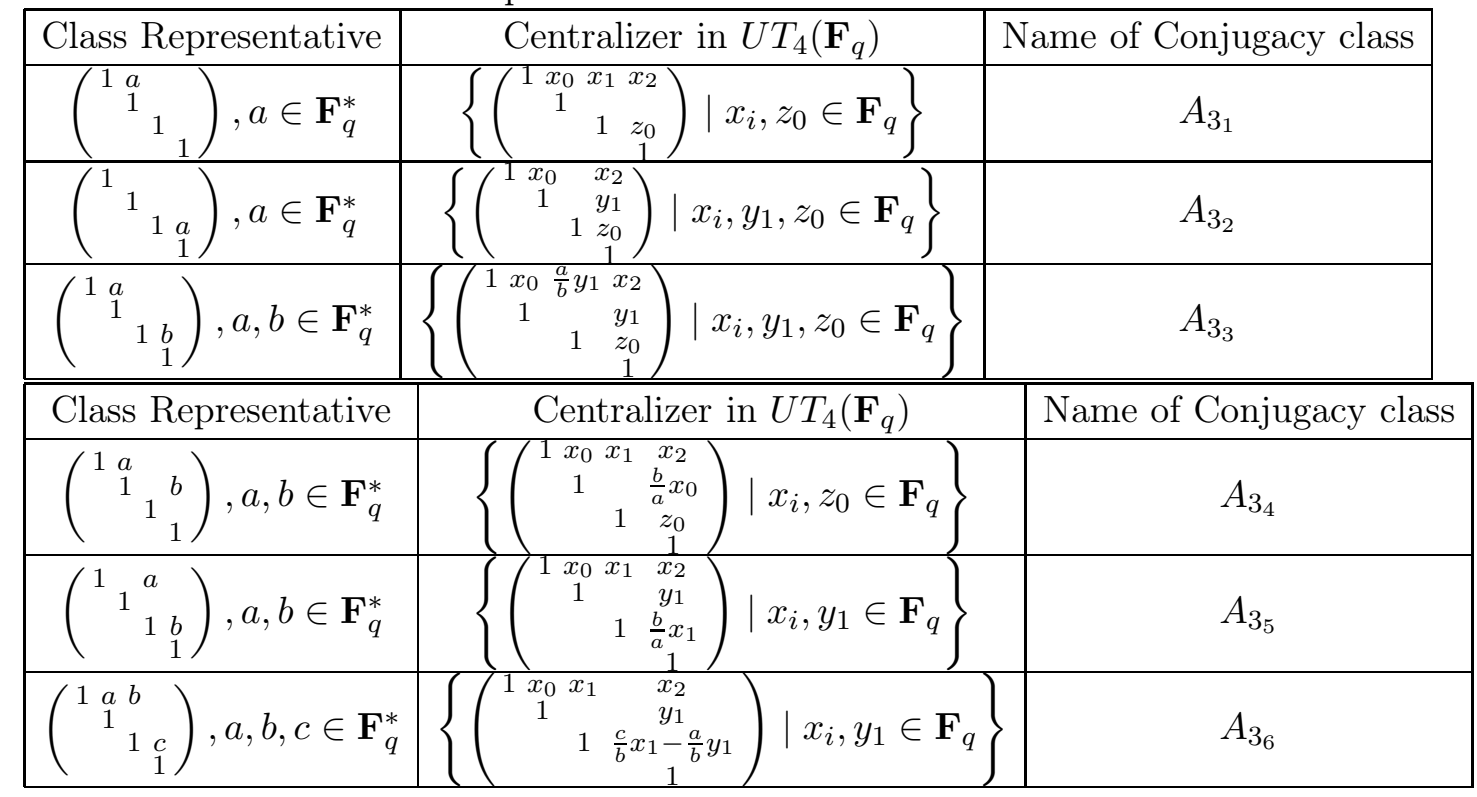

(1) The following map gives isomorphism between centralizers of representative of conjugacy classes $A_{3_{1}}$ and $A_{3_{2}}$.

$$
\left(\begin{array}{cccc}
1 & x_{0} & & x_{2} \\
& 1 & & y_{1} \\
& & 1 & z_{0} \\
& & & 1
\end{array}\right) \mapsto\left(\begin{array}{cccc}
1 & z_{0} & y_{1} & x_{2}-y_{1} x_{0} \\
& 1 & & \\
& & 1 & -x_{0} \\
& & & 1
\end{array}\right)
$$


(2) The following map gives isomorphism between centralizers of representative of conjugacy classes $A_{3_{1}}$ and $A_{3_{4}}$.

$$
\left(\begin{array}{cccc}
1 & x_{0} & x_{1} & x_{2} \\
& 1 & & \\
& & 1 & z_{0} \\
& & & 1
\end{array}\right) \mapsto\left(\begin{array}{cccc}
1 & x_{0} & x_{1} & x_{2}-\left(\frac{x_{0}\left(x_{0}-1\right)}{2}\right) \lambda \\
& 1 & & \lambda x_{0} \\
& & 1 & z_{0} \\
& & & 1
\end{array}\right)
$$

(3) The following map gives isomorphism between centralizers of representative of conjugacy classes $A_{32}$ and $A_{35}$.

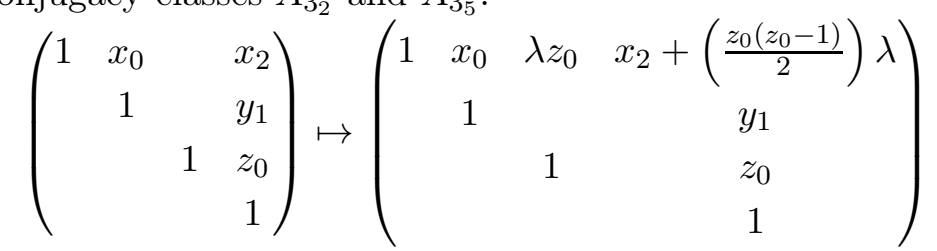

(4) The following map gives isomorphism between centralizers of representative of conjugacy classes $A_{3_{2}}$ and $A_{3_{3}}$.

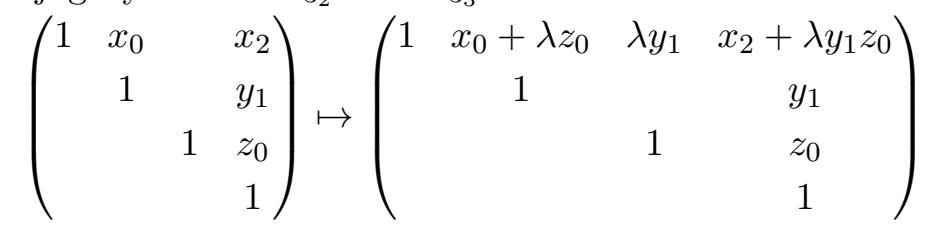

(5) The following map gives isomorphism between centralizers of representative of conjugacy classes $A_{32}$ and $A_{3_{6}}$.

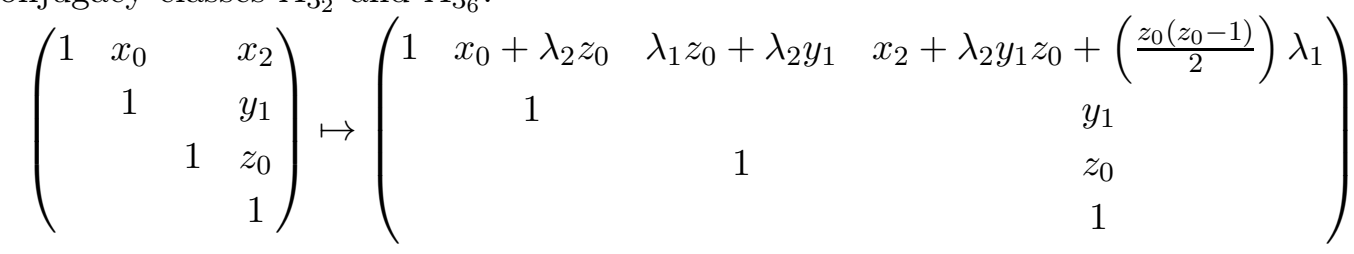

\section{Branching RUles FOR $U T_{5}\left(\mathbf{F}_{q}\right)$}

In this section, we will discuss the simultaneous conjugacy classes of tuples of commuting matrices of $U T_{5}\left(\mathbf{F}_{q}\right)$. The types are listed in Section $\mathrm{B}$. The branching matrix is as follows:

Theorem 7.1. The branching rule of $U T_{5}\left(\mathbf{F}_{q}\right)$ has 3 new types. The branching matrix $B_{U T_{5}\left(\mathbf{F}_{q}\right)}$ is in table 4 which is a $20 \times 20$ matrix.

Once again it's easy to see the branches for central and regular types. 
TABLE 4. Branching matrix of $U T_{5}\left(\mathbf{F}_{q}\right)$

\begin{tabular}{|c|c|c|c|c|c|c|c|c|c|c|c|c|c|c|c|c|c|c|c|}
\hline$C$ & $A_{1}$ & $A_{2}$ & $A_{3}$ & $A_{4}$ & $A_{5}$ & $B_{1}$ & $B_{2}$ & $B_{3}$ & $B_{4}$ & $B_{5}$ & $B_{6}$ & $D_{1}$ & $D_{2}$ & $R_{1}$ & $R_{2}$ & $R_{3} L$ & $U N T_{1}$ & $U N T_{2}$ & $U N T_{3}$ \\
\hline$q$ & 0 & 0 & 0 & 0 & 0 & 0 & 0 & 0 & 0 & 0 & 0 & 0 & 0 & 0 & 0 & 0 & 0 & 0 & 0 \\
\hline $2(q-1)$ & $q^{2}$ & 0 & 0 & 0 & 0 & 0 & 0 & 0 & 0 & 0 & 0 & 0 & 0 & 0 & 0 & 0 & 0 & 0 & 0 \\
\hline$q^{2}-q$ & $q\left(q^{2}-1\right)$ & $q^{4}$ & 0 & 0 & 0 & $3 q^{2}-3 q$ & 0 & 0 & 0 & 0 & 0 & 0 & 0 & 0 & 0 & 0 & 0 & 0 & 0 \\
\hline $2 q^{2}-2 q$ & 0 & 0 & $q^{2}$ & 0 & 0 & 0 & 0 & 0 & 0 & 0 & 0 & 0 & 0 & 0 & 0 & 0 & 0 & 0 & 0 \\
\hline $2 q^{2}-2 q$ & $2 q^{2}(q-1)$ & 0 & $q\left(q^{2}-1\right)$ & $q^{4}$ & 0 & 0 & $q^{3}-q$ & 0 & 0 & 0 & 0 & 0 & 0 & 0 & 0 & 0 & 0 & 0 & 0 \\
\hline $\begin{array}{c}\left(q^{2}-1\right) \\
(2 q-1)\end{array}$ & 0 & 0 & 0 & 0 & $q^{2}$ & 0 & 0 & 0 & 0 & 0 & 0 & 0 & 0 & 0 & 0 & 0 & 0 & 0 & 0 \\
\hline$(q-1)^{2}$ & 0 & 0 & 0 & 0 & 0 & $q^{2}$ & 0 & 0 & 0 & 0 & 0 & 0 & 0 & 0 & 0 & 0 & 0 & 0 & 0 \\
\hline $2 q^{2}-2 q$ & 0 & 0 & 0 & 0 & 0 & 0 & $q^{2}$ & 0 & 0 & 0 & 0 & 0 & 0 & 0 & 0 & 0 & 0 & 0 & 0 \\
\hline $2(q-1)^{2}$ & $q^{2}(q-1)$ & 0 & $q^{2}(q-1)$ & 0 & 0 & 0 & 0 & $q^{3}$ & 0 & 0 & 0 & 0 & 0 & 0 & 0 & 0 & 0 & 0 & 0 \\
\hline $\begin{array}{c}\left(2 q^{2}+4\right) . \\
(q-1)^{2}\end{array}$ & $\begin{array}{c}q(q-1) \\
\left(q^{3}+q^{2}-1\right)\end{array}$ & 0 & 0 & 0 & $2 q(q-1)$ & 0 & $q^{2}-q$ & 0 & $q^{3}$ & 0 & 0 & $2 q(q-1)$ & 0 & 0 & 0 & 0 & 0 & 0 & 0 \\
\hline$q(q-1)^{2}$ & 0 & 0 & $q^{3}(q-1)$ & 0 & 0 & 0 & 0 & 0 & 0 & $q^{2}$ & 0 & 0 & 0 & 0 & 0 & 0 & 0 & 0 & 0 \\
\hline $2 q(q-1)^{2}$ & 0 & 0 & 0 & 0 & $q^{2}(q-1)$ & $q^{2}(q-1)$ & $q^{4}-q^{3}$ & 0 & 0 & $\begin{array}{l}\left(q^{3}+q\right) . \\
\left(q^{2}-1\right)\end{array}$ & $q^{3}$ & 0 & 0 & 0 & 0 & 0 & 0 & 0 & 0 \\
\hline$(q-1)^{3}$ & 0 & 0 & 0 & 0 & 0 & 0 & 0 & 0 & 0 & 0 & 0 & $q^{2}$ & 0 & 0 & 0 & 0 & 0 & 0 & 0 \\
\hline $\begin{array}{c}(2 q+1) \\
(q-1)^{3}\end{array}$ & 0 & 0 & 0 & 0 & 0 & 0 & 0 & 0 & 0 & 0 & 0 & 0 & $q^{3}$ & 0 & 0 & 0 & 0 & 0 & 0 \\
\hline $2(q-1)^{2}$ & $2 q^{3}-2 q^{2}$ & $2 q^{4}-2 q^{2}$ & $\begin{array}{c}q(q-1) . \\
\left(q^{2}+q-1\right)\end{array}$ & 0 & 0 & $2 q^{3}-4 q+2$ & $\begin{array}{c}\left(q^{2}-q\right) \\
\left(q^{2}+q-1\right)\end{array}$ & $\begin{array}{c}q^{2}(q-1) \\
\left(q^{2}+q-1\right)\end{array}$ & 0 & 0 & 0 & 0 & 0 & $q^{6}$ & 0 & 0 & 0 & $q^{5}-q^{2}$ & 0 \\
\hline$q(q-1)^{2}$ & $\begin{array}{c}q(q-1)^{2} \\
(q+1)\end{array}$ & $q\left(q^{2}-1\right)^{2}$ & $\begin{array}{c}q\left(q^{2}-1\right) \\
(q-1)\end{array}$ & $q^{4}(q-1)$ & $q^{2}(q-1)$ & $\begin{array}{c}q(q-1)^{2} \\
(q+2)\end{array}$ & $\begin{array}{l}(q-1) \\
\left(q^{3}-q\right)\end{array}$ & 0 & $q^{4}-q^{2}$ & 0 & 0 & 0 & 0 & 0 & $q^{5}$ & 0 & $q^{4}-q^{2}$ & $q^{4}-q^{3}$ & $q^{4}-q^{2}$ \\
\hline $\begin{array}{l}\left(q^{2}-1\right) \\
(q-1)^{2}\end{array}$ & $q^{2}(q-1)^{2}$ & 0 & $q^{2}(q-1)^{2}$ & $q^{3}\left(q^{2}-1\right)$ & $\begin{array}{r}q(q-1) \\
\left(q^{2}-1\right)\end{array}$ & $\begin{array}{c}(q-1)^{2} \\
\left(q^{2}+q+1\right)\end{array}$ & $q^{2}(q-1)^{2}$ & $q^{3}(q-1)$ & 0 & $q^{4}-q^{3}$ & $q^{2}\left(q^{2}-1\right)$ & $q^{2}\left(q^{2}-1\right)$ & $q^{2}\left(q^{2}-1\right)$ & 0 & 0 & $q^{4}$ & $q^{4}-q^{3}$ & $q^{4}-q^{3}$ & $q^{4}-q^{3}$ \\
\hline 0 & 0 & 0 & $q^{2}(q-1)$ & 0 & $q(q-1)^{2}$ & $(q-1)^{2}$ & $q(q-1)^{2}$ & 0 & 0 & 0 & 0 & 0 & 0 & 0 & 0 & 0 & $q^{3}$ & 0 & 0 \\
\hline 0 & 0 & 0 & 0 & 0 & 0 & $2 q(q-1)$ & $q^{3}-q^{2}$ & 0 & 0 & 0 & 0 & 0 & 0 & 0 & 0 & 0 & 0 & $q^{3}$ & 0 \\
\hline 0 & 0 & 0 & 0 & 0 & 0 & $(q-1)^{3}$ & 0 & 0 & 0 & 0 & 0 & $q(q-1)^{2}$ & 0 & 0 & 0 & 0 & 0 & 0 & $q^{3}$ \\
\hline
\end{tabular}




\subsection{Branching of type $A$.}

Proposition 7.2. An upper unitriangular matrix of type $A_{1}$ has the following branches:

\begin{tabular}{cc|cc}
\hline Branch & No. of Branches & Branch & No. of Branches \\
\hline$A_{1}$ & $q^{2}$ & $B_{4}$ & $q(q-1)\left(q^{3}+q^{2}-1\right)$ \\
$A_{2}$ & $q\left(q^{2}-1\right)$ & $R_{1}$ & $2 q^{2}(q-1)$ \\
$A_{4}$ & $2 q^{2}(q-1)$ & $R_{2}$ & $q(q-1)^{2}(q+1)$ \\
$B_{3}$ & $q^{2}(q-1)$ & $R_{3}$ & $q^{2}(q-1)^{2}$ \\
\hline
\end{tabular}

Proof. For a matrix of type $A_{1}$, there are two canonical forms: $I_{5}+a E_{14}$, and $I_{5}+a E_{25}$, where $a \neq 0$. We will take our matrix $A$ of type $A_{1}$, to be the canonical form $I_{5}+a E_{14}$, $a \neq 0$. So the centralizer of $A$ is $Z_{U T_{5}\left(\mathbf{F}_{q}\right)}(A)=\left\{\left(\begin{array}{ccccc}1 & a_{1} & b_{1} & b_{2} & a_{2} \\ & 1 & c_{1} & c_{2} & d_{1} \\ & & 1 & c_{3} & d_{2} \\ & & 1 & \\ & & & 1\end{array}\right)\right\}$. Let $B=$

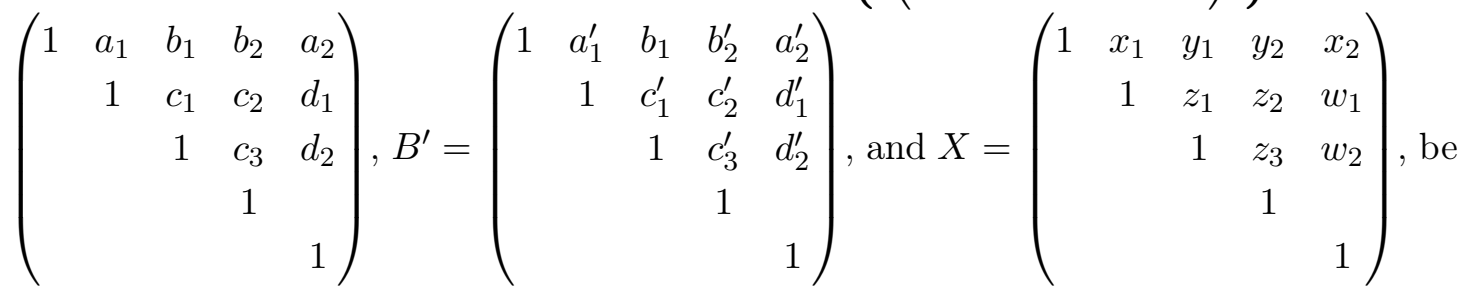
such that $X B=B^{\prime} X$. From $X B=B^{\prime} X$, we get that $a_{1}^{\prime}=a_{1}$. Let $C$ denote the middle $3 \times 3$ unitriangular block $\left(\begin{array}{ccc}1 & c_{1} & c_{2} \\ & 1 & c_{3} \\ & & 1\end{array}\right)$ in the matrix $B$, and let $Z$ denote the middle block, $\left(\begin{array}{ccc}1 & z_{1} & z_{2} \\ & 1 & z_{3} \\ & & 1\end{array}\right)$, from $X$. Likewise, we have $C^{\prime}$. We see that from $X B=B^{\prime} X$, we have $Z C=C^{\prime} Z$. Thus we take $C$ to be a conjugacy class representative from $U T_{3}\left(\mathbf{F}_{q}\right)$, and $Z$ to be its centralizer element in $U T_{3}\left(\mathbf{F}_{q}\right)$. Now, with this, we have the following set of equations:

$$
\begin{aligned}
& \left(\begin{array}{lll}
x_{1} & y_{1} & y_{2}
\end{array}\right) C+\left(\begin{array}{lll}
a_{1} & b_{1} & b_{2}
\end{array}\right)=\left(\begin{array}{lll}
a_{1} & b_{1}^{\prime} & b_{2}^{\prime}
\end{array}\right) Z+\left(\begin{array}{lll}
x_{1} & y_{1} & y_{2}
\end{array}\right) \\
& Z\left(\begin{array}{c}
d_{1} \\
d_{2} \\
0
\end{array}\right)+\left(\begin{array}{c}
w_{1} \\
w_{2} \\
0
\end{array}\right)=C\left(\begin{array}{c}
w_{1} \\
w_{2} \\
0
\end{array}\right)+\left(\begin{array}{c}
d_{1}^{\prime} \\
d_{2}^{\prime} \\
0
\end{array}\right) \\
& x_{1} d_{1}+y_{1} d_{2}+a_{2}=a_{1} w_{1}+b_{1}^{\prime} w_{2}+a_{2}^{\prime}
\end{aligned}
$$

We look at two main cases, $a_{1}=0$, and $a_{1} \neq 0$. 
Case $a_{1}=0$ : Here Equation 7.3 is reduced to $x_{1} d_{1}+y_{1} d_{2}+a_{2}=b_{1}^{\prime} w_{2}+a_{2}^{\prime}$. Here we look at subcases:

When $\left(b_{1}, b_{2}\right)=\left(d_{1}, d_{2}\right)=(0,0)$ : Thus Equations 7.1 and 7.2 become:

$$
\begin{aligned}
\left(\begin{array}{rll}
x_{1} & y_{1} & y_{2}
\end{array}\right) C & =\left(\begin{array}{lll}
x_{1} & y_{1} & y_{2}
\end{array}\right) \\
\left(\begin{array}{c}
w_{1} \\
w_{2} \\
0
\end{array}\right) & =C\left(\begin{array}{c}
w_{1} \\
w_{2} \\
0
\end{array}\right)
\end{aligned}
$$

and $a_{2}^{\prime}=a_{2}$.

When $C=I_{3}$ : Equations 7.1 and 7.2 are void, and $B$ is reduced to $\left(\begin{array}{ccccc}1 & & & & a_{2} \\ & 1 & & & \\ & 1 & & \\ & & 1 & \\ & & & & 1\end{array}\right)$.

Thus $Z_{U T_{5}\left(\mathbf{F}_{q}\right)}(A, B)=Z_{U T_{5}\left(\mathbf{F}_{q}\right)}(A)$. So $(A, B)$ is a branch of type $A_{1}$, and there are $q$ branches.

When $C=\left(\begin{array}{lll}1 & & c \\ & 1 & \\ & & 1\end{array}\right), c \neq 0$ : Equation 7.2 remains void, but from Equation 7.1 , we get $c x_{1}+y_{2}=y_{2}$, which leaves us with $x_{1}=0$, as $c \neq 0$. Thus the branch is

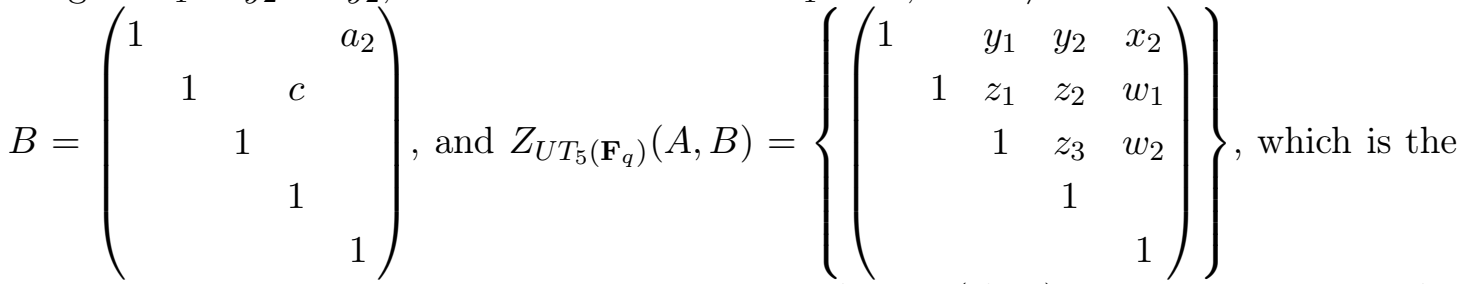
centralizer of one of the canonical forms of type $A_{2}$. So $(A, B)$ is a branch of type $A_{2}$, and there are $q(q-1)$ such branches.

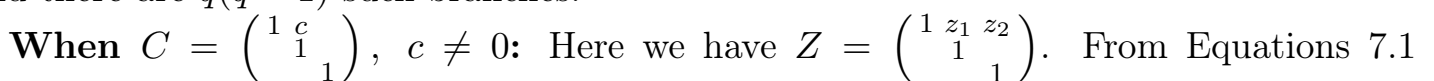
and 7.2, we have $c x_{1}+y_{1}=y_{1}$ and $w_{1}+c w_{2}=w_{1}$, thus we have $x_{1}=w_{2}=0$. So we have $B=\left(\begin{array}{ccccc}1 & & & & a_{2} \\ & 1 & c & & \\ & & 1 & & \\ & & & 1 & \\ & & & & 1\end{array}\right)$, and $Z_{U T_{5}\left(\mathbf{F}_{q}\right)}(A, B)=\left\{\left(\begin{array}{ccccc}1 & & y_{1} & y_{2} & x_{2} \\ & 1 & z_{1} & z_{2} & w_{1} \\ & & 1 & & \\ & & 1 & \\ & & & & 1\end{array}\right)\right\}$, and by a routine check, we see that $Z_{U T_{5}\left(\mathbf{F}_{q}\right)}(A, B)$ is commutative, of size $q^{6} .(A, B)$ is of the regular type $R_{1}$, and there are $(q-1) q$ branches of this type.

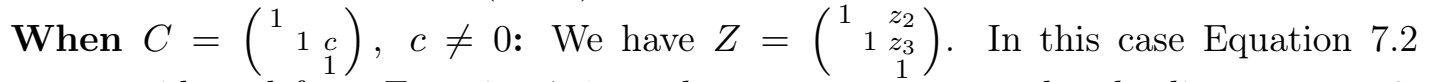
becomes void, and from Equation [7.1, we have $c y_{1}+y_{2}=y_{2}$, thus leading to $y_{1}=0$. 
Hence, $B=\left(\begin{array}{ccccc}1 & & & & a_{2} \\ & 1 & & & \\ & & 1 & c & \\ & & & 1 & \\ & & & & 1\end{array}\right)$. We have $Z_{U T_{5}\left(\mathbf{F}_{q}\right)}(A, B)=\left\{\left(\begin{array}{ccccc}1 & x_{1} & & y_{2} & x_{2} \\ & 1 & & z_{2} & w_{1} \\ & & 1 & z_{3} & w_{2} \\ & & & 1 & \\ & & & & 1\end{array}\right)\right\}$,

which is the centralizer of a unitriangular matrix of type $A_{4}$. So $(A, B)$ is a branch of type $A_{4}$, and there are $q(q-1)$ branches.

When $C=\left(\begin{array}{ccc}1 & c_{1} & \\ & 1 & c_{2} \\ & & 1\end{array}\right), c_{1}, c_{2} \neq 0$ : We have $Z=\left(\begin{array}{ccc}1 & z_{1} & z_{2} \\ 1 & \lambda z_{1} \\ & 1\end{array}\right)$, where $\lambda=c_{2} / c_{1}$. From Equation 17.1, we have $c_{1} x_{1}+y_{1}=y_{1}$, which leaves us with $x_{1}=0$, and then we have $c_{2} y_{1}+y_{2}=y_{2}$, which leaves us with $y_{1}=0$. Then, from Equation [7.2, we have $w_{1}+c_{1} w_{2}=w_{1}$, leaving us with with $w_{2}=0$. So, we have $B=\left(\begin{array}{cccccc}1 & & & & a_{2} \\ & 1 & c_{1} & & \\ & 1 & c_{2} & \\ & & & 1 & \\ & & & & 1\end{array}\right)$, and $Z_{U T_{5}\left(\mathbf{F}_{q}\right)}(A, B)=\left\{\left(\begin{array}{ccccc}1 & & & y_{2} & x_{2} \\ & 1 & z_{1} & z_{2} & w_{1} \\ & & 1 & \lambda z_{1} & \\ & & & 1 & \\ & & & & 1\end{array}\right)\right\}$. This is of size $q^{5}$, and by a routine check, it can be seen that $Z_{U T_{5}\left(\mathbf{F}_{q}\right)}(A, B)$ is commutative. Thus $(A, B)$ is of the regular type $R_{2}$, and there are $q(q-1)^{2}$ branches.

When $\left(\left(b_{1}, b_{2}\right),\left(d_{1}, d_{2}\right)\right) \neq((0,0),(0,0))$ : We shall start with $C=I_{3}$.

When $C=I_{3}$ : Here $Z$ is any aribtrary matrix in $U T_{3}\left(\mathbf{F}_{q}\right)$, and Equations 7.1 and 7.2 become:

$$
\begin{aligned}
\left(\begin{array}{lll}
0 & b_{1} & b_{2}
\end{array}\right) & =\left(\begin{array}{lll}
0 & b_{1}^{\prime} & b_{2}^{\prime}
\end{array}\right)\left(\begin{array}{ccc}
1 & z_{1} & z_{2} \\
0 & 1 & z_{3} \\
0 & 0 & 1
\end{array}\right) \\
\left(\begin{array}{ccc}
1 & z_{1} & z_{2} \\
0 & 1 & z_{3} \\
0 & 0 & 1
\end{array}\right)\left(\begin{array}{c}
d_{1} \\
d_{2} \\
0
\end{array}\right) & =\left(\begin{array}{c}
d_{1}^{\prime} \\
d_{2}^{\prime} \\
0
\end{array}\right)
\end{aligned}
$$

From the above equation, we have $b_{1}^{\prime}=b_{1}$, and $b_{2}=b_{2}^{\prime}+b_{1} z_{3}$, and we have $d_{1}+z_{1} d_{2}=$ $d_{1}^{\prime}$, and $d_{2}^{\prime}=d_{2}$.

Firstly, if both $b_{1} \neq 0$ and $d_{2} \neq 0$. Then we can choose a $z_{3}$ such that $b_{2}^{\prime}=0$, and similarly we can choose $z_{1}$ such that $d_{1}^{\prime}=0$. Hence, with this Equation 7.3 is reduced to $y_{1} d_{2}+a_{2}=b_{1} w_{2}+a_{2}^{\prime}$. We may choose a $w_{2}$ such that $d_{2}^{\prime}=0$. Thus, we have reduced $B$ 
to $\left(\begin{array}{ccccc}1 & & b_{1} & & \\ & 1 & & & \\ & & 1 & & d_{2} \\ & & & 1 & \\ & & & & 1\end{array}\right)$, and $Z_{U T_{5}\left(\mathbf{F}_{q}\right)}(A, B)=\left\{\left(\begin{array}{ccccc}1 & x_{1} & y_{1} & y_{2} & x_{2} \\ & 1 & & z_{2} & w_{1} \\ & 1 & & \lambda y_{1} \\ & & 1 & \\ & & & & 1\end{array}\right) \mid \lambda=d_{2} / b_{1}\right\}$.

This is the centralizer of a matrix of type $A_{4}$. Thus, we have $(q-1)^{2}$ branches of this type.

When $b_{1} \neq 0$ and $d_{2}=0$, we again pick a $z_{3}$ such that $b_{2}^{\prime}=0$, and Equation 7.3 is reduced to $x_{1} d_{1}+a_{2}=b_{1} w_{2}+a_{2}^{\prime}$. Again, choose $w_{2}$ so that $a_{2}^{\prime}=0$. Thus $B$ is reduced to $\left(\begin{array}{ccccc}1 & & b_{1} & & \\ & 1 & & & d_{1} \\ & & 1 & & \\ & & & 1 & \\ & & & & 1\end{array}\right)$, and $Z_{U T_{5}\left(\mathbf{F}_{q}\right)}(A, B)=\left\{\left(\begin{array}{ccccc}1 & x_{1} & y_{1} & y_{2} & x_{2} \\ & 1 & z_{1} & z_{2} & w_{1} \\ & & & \lambda x_{1} \\ & & 1 & \\ & & & & 1\end{array}\right) \mid \lambda=d_{1} / b_{1}\right\}$, which is isomorphic (conjugation by the matrix that swaps the 4th and 5th rows and columns) to centralizer of a matrix of type $B_{3}$. Thus there are $q(q-1)$ branches of this type.

When $b_{1}=0$, we have $b^{\prime} 2_{2}=b_{2}$. We consider $d_{2} \neq 0$, and choose a suitable $z_{1}$ so that $d_{1}^{\prime}=0$. Equation 7.3 is reduced to $y_{1} d_{2}+a_{2}=a_{2}^{\prime}$. Thus, we choose an apporpriate $y_{1}$ so that $a_{2}^{\prime}=0$. $B$ is thus reduced to $\left(\begin{array}{ccccc}1 & & b_{2} & & \\ & 1 & & & \\ & & & d_{2} \\ & & 1 & \\ & & & 1\end{array}\right)$, and $Z_{U T_{5}\left(\mathbf{F}_{q}\right)}(A, B)=$

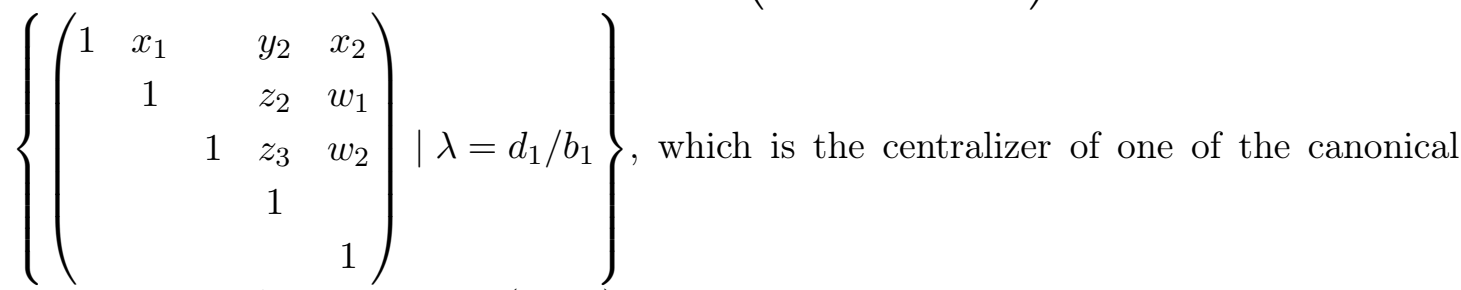
forms of type $A_{4}$. There are $q(q-1)$ such branches.

When $b_{2} \neq 0, d_{2}=0$, we have $d_{1}^{\prime}=d_{1}$. We first take $d_{1}=0$. Then Equation 7.3 is reduced to $a_{2}^{\prime}=a_{2}$. We thus have $B$ reduced to $\left(\begin{array}{lllll}1 & & & b_{2} & a_{2} \\ & 1 & & & \\ & 1 & & \\ & & 1 & \\ & & & 1\end{array}\right)$, and thus $Z_{U T_{5}\left(\mathbf{F}_{q}\right)}(A, B)=Z_{U T_{5}\left(\mathbf{F}_{q}\right)}(A)$. Hence, $(A, B)$ is a branch of type $A_{1}$, and there are $q(q-1)$ branches. 
When $b_{1}=0$, with $d_{2}=0$, and $d_{1} \neq 0$. Equation 7.3 is reduced to $x_{1} d_{1}+a_{2}=a_{2}^{\prime}$. With a suitable $x_{1}$, we can get rid of $d_{1}$. Hence $B$ is reduced to $\left(\begin{array}{ccccc}1 & & & b_{2} & \\ & & & & d_{1} \\ & 1 & & \\ & & 1 & \\ & & & & 1\end{array}\right)$, and $Z_{U T_{5}\left(\mathbf{F}_{q}\right)}(A, B)=\left\{\left(\begin{array}{ccccc}1 & & y_{1} & y_{2} & x_{2} \\ & 1 & z_{1} & z_{2} & w_{1} \\ & & 1 & z_{3} & w_{2} \\ & & & 1 & \\ & & & & 1\end{array}\right)\right\}$. Thus $(A, B)$ is of type $A_{2}$, and there are $q(q-1)$ such branches.

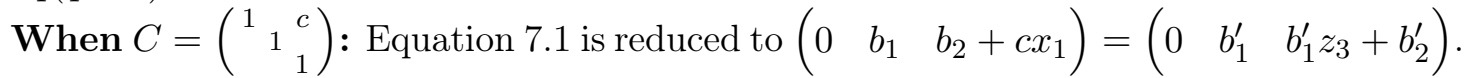
Thus, we have $b_{1}^{\prime}=b_{1}$, and we can choose $x_{1}$ such that $b_{2}^{\prime}=0$. Now, here, on replacing $b_{2}^{\prime}$ and $b_{2}$ by 0 in the above equation, we get that $x_{1}=\frac{b_{1}}{c} z_{3}$. From Equation 7.2 , we have $d_{2}^{\prime}=d_{2}$, and $d_{1}^{\prime}=d_{1}+z_{1} d_{2}$. Equation 7.3 becomes $\frac{b_{1}}{c} z_{3} d_{1}+y_{1} d_{2}+a_{2}=w_{2} b_{1}+a_{2}^{\prime}$.

We now look at the case when $b_{1} \neq 0$, and $d_{2}^{\prime} \neq 0$. We choose $z_{1}$ so that $d_{1}^{\prime}=0$, and $w_{2}$ such that $a_{2}^{\prime}=0$. Hence, we reduce $B$ to $\left(\begin{array}{ccccc}1 & & b_{1} & & \\ & 1 & & c & \\ & & & & d_{2} \\ & & 1 & \\ & & & 1\end{array}\right)$, and we have $Z_{U T_{5}\left(\mathbf{F}_{q}\right)}(A, B)=\left\{\left(\begin{array}{ccccc}1 & \lambda z_{3} & y_{1} & y_{2} & x_{2} \\ & 1 & & z_{2} & w_{1} \\ & & 1 & z_{3} & \mu y_{1} \\ & & & 1 & \\ & & & & 1\end{array}\right) \mid \lambda=\frac{b_{1}}{c}, \mu=\frac{d_{2}}{b_{1}}\right\}$, which is isomorphic to the centralizer of some canonical matrix of type $B_{4}$. There are $(q-1)^{3}$ such branches.

When $b_{1} \neq 0$, and $d_{2}=0$, then $d_{1}^{\prime}=d_{1}$. Equation 7.3 becomes $\frac{b_{1}}{c} z_{3} d_{1}+a_{2}=w_{2} b_{1}+a_{2}^{\prime}$. Choose a suitable $w_{2}$, to make $a_{2}^{\prime}=0$. Then $B$ is reduced to $\left(\begin{array}{ccccc}1 & & b_{1} & & \\ & & & c & d_{1} \\ & 1 & & \\ & & 1 & \\ & & & 1\end{array}\right)$, 
and $Z_{U T_{5}\left(\mathbf{F}_{q}\right)}=\left\{\left(\begin{array}{ccccc}1 & \lambda z_{3} & y_{1} & y_{2} & x_{2} \\ & 1 & z_{1} & z_{2} & w_{1} \\ & & 1 & z_{3} & \mu z_{3} \\ & & & 1 & \\ & & & & 1\end{array}\right) \mid \lambda=\frac{b_{1}}{c}, \mu=\frac{d_{1}}{c}\right\}$. If we write $z_{3}$ in terms

of $z_{1}$, then $Z_{U T_{5}\left(\mathbf{F}_{q}\right)}$ will be this: $\left\{\left(\begin{array}{ccccc}1 & x_{1} & y_{1} & y_{2} & x_{2} \\ & 1 & z_{1} & z_{2} & w_{1} \\ & & 1 & \lambda x_{1} & \mu x_{1} \\ & & & 1 & \\ & & & & 1\end{array}\right) \mid \lambda=\frac{c}{b_{1}}, \mu=\frac{d_{1}}{b_{1}}\right\}$. If we

conjugate this centralizer by the matrix $I+\frac{\mu}{\lambda} E_{45}$, we get the centralizer of a canonical unitriangular matrix of type $B_{3}$. Thus $(A, B)$ is a branch of type $B_{3}$, and there are $q(q-1)^{2}$ such branches.

Now, when $b_{1}=0$, and $\left(d_{1}, d_{2}\right) \neq(0,0)$. We have $x_{1}=\frac{b_{1}}{c} z_{3}=0$, a nd Equation 7.3 becomes $y_{1} d_{2}+a_{2}=a_{2}^{\prime}$. First, when $d_{2} \neq 0$, then we choose $z_{1}$ so that $d_{1}^{\prime}=0$, and choose $y_{1}$ so that $a_{2}^{\prime}=0$. So, $B$ is reduced to $\left(\begin{array}{ccccc}1 & & & & \\ & 1 & & c & \\ & 1 & & d_{2} \\ & & 1 & \\ & & & 1\end{array}\right)$, and $Z_{U T_{5}\left(\mathbf{F}_{q}\right)}=$

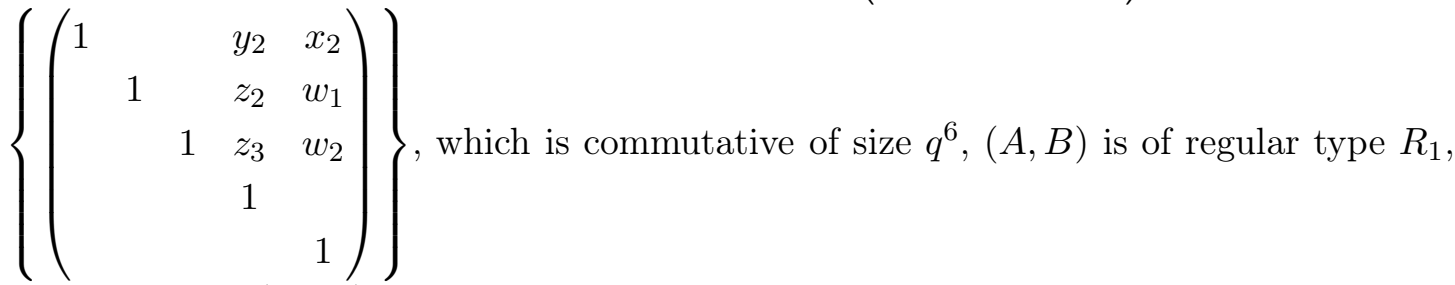
and there are $q(q-1)$ such branches.

When $b_{1}=d_{2}=0, d_{1} \neq 0$. We have $d_{1}^{\prime}=d_{1}$, and Equation 7.3 reduces to $a_{2}^{\prime}=a_{2}$. Thus, $B$ is reduced to $\left(\begin{array}{ccccc}1 & & & & a_{2} \\ & 1 & & c & d_{1} \\ & & & & \\ & & & 1 & \\ & & & & \\ & & & & \end{array}\right)$, and $Z_{U T_{5}\left(\mathbf{F}_{q}\right)}=\left(\begin{array}{ccccc}1 & & & a_{2} \\ & 1 & & c & \\ & 1 & & \\ & & 1 & \\ & & & 1\end{array}\right)$, and $Z_{U T_{5}\left(\mathbf{F}_{q}\right)}(A, B)=\left\{\left(\begin{array}{ccccc}1 & & y_{1} & y_{2} & x_{2} \\ & 1 & z_{1} & z_{2} & w_{1} \\ & & 1 & z_{3} & w_{2} \\ & & & 1 & \\ & & & & 1\end{array}\right)\right\}$, which is the centralizer of a matrix of type $A_{2}$. Thus $(A, B)$ is of type $A_{2}$, and there are $q(q-1)^{2}$ such branches. 


$$
\begin{gathered}
\text { When } C=\left(\begin{array}{ccc}
1 & c & \\
& 1 & \\
& & 1
\end{array}\right), c \neq 0 \text { : Here } Z=\left(\begin{array}{ccc}
1 & z_{1} & z_{2} \\
& 1 & \\
& & 1
\end{array}\right) \text {, and Equation } 7.1 \text { becomes: } \\
\left(\begin{array}{ccc}
c x_{1}+b_{1} & b_{2}
\end{array}\right)=\left(\begin{array}{ll}
b_{1}^{\prime} & b_{2}^{\prime}
\end{array}\right) .
\end{gathered}
$$

Using a nice $x_{1}$, we can make $b_{1}^{\prime}=0$, and $b_{2}^{\prime}=b_{2}$. So, if we replace $b_{1}$ by $b_{1}^{\prime}=0$ in the above equation, we have $x_{1}=0$. Next, Equation 7.2 becomes:

$$
\left(\begin{array}{c}
d_{1}+z_{1} d_{2} \\
d_{2} \\
0
\end{array}\right)=\left(\begin{array}{c}
c w_{2}+d_{1}^{\prime} \\
d_{2}^{\prime} \\
0
\end{array}\right)
$$

As $c \neq 0$, we choose a $w_{2}$ so that $d_{1}^{\prime}=0$. We have $d_{2}^{\prime}=d_{2}$. With these, Equation 7.3 becomes

When $d_{2} \neq 0$, choose $y_{1}$ such that $a_{2}^{\prime}=0$. $B$ is reduced to $\left(\begin{array}{lllll}1 & & & b_{2} & \\ & 1 & c & & \\ & & 1 & & d_{2} \\ & & 1 & \\ & & & & 1\end{array}\right)$, and $Z_{U T_{5}\left(\mathbf{F}_{q}\right)}(A, B)=\left\{\left(\begin{array}{ccccc}1 & & & y_{2} & x_{2} \\ & 1 & z_{1} & z_{2} & w_{1} \\ & & 1 & & \lambda z_{1} \\ & & & 1 & \\ & & & & 1\end{array}\right) \mid \lambda=\frac{d_{2}}{c}\right\}$. Thus $(A, B)$ is of regular type $R_{2}$, and there are $q(q-1)^{2}$ branches of this type.

When $d_{2}=0$, then we are left with $b_{2} \neq 0$. Hence Equation 7.3 is reduced to $a_{2}^{\prime}=a_{2}$. Hence $B$ is reduced to $\left(\begin{array}{ccccc}1 & & & b_{2} & a_{2} \\ & 1 & c & & \\ & & 1 & & \\ & & & 1 & \\ & & & & 1\end{array}\right)$, and $Z_{U T_{5}\left(\mathbf{F}_{q}\right)}(A, B)=\left\{\left(\begin{array}{ccccc}1 & & y_{1} & y_{2} & x_{2} \\ & 1 & z_{1} & z_{2} & w_{1} \\ & 1 & & \\ & & 1 & \\ & & & & 1\end{array}\right)\right\}$, which is the centralizer of a matrix of type $R_{1}$. $(A, B)$ is a branch of type $R_{1}$, and there are $(q-1)^{2} q$ such branches.

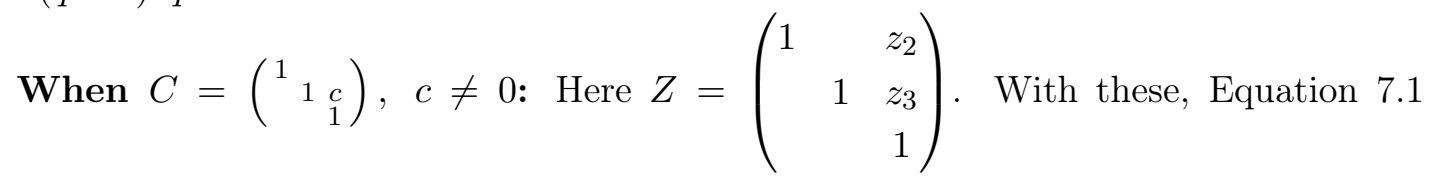
becomes:

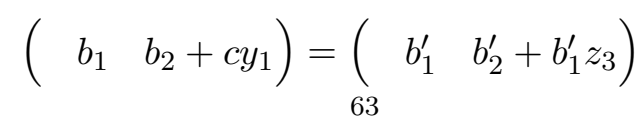


So, we have $b_{1}^{\prime}=b_{1}$, and we can choose $y_{1}$ so that $b_{2}^{\prime}=0$. Thus, on equating the above equation, with $b_{2}$ replaced by 0 , we get that $y_{1}=\frac{b_{1}}{c} z_{3}$; and from Equation 7.2 , we have $d_{1}^{\prime}=d_{1}$, and $d_{2}^{\prime}=d_{2}$, and thus Equation 7.3 boils down to $x_{1} d_{1}+\frac{b_{1}}{c} z_{3} \cdot d_{1}=b_{1} w_{2}+a_{2}^{\prime}$. We first look at the case, when $b_{1} \neq 0$. Then choose $w_{2}$ so that $a_{2}^{\prime}=0$. So $B$ reduces to $\left(\begin{array}{ccccc}1 & & b_{1} & \\ & 1 & & & d_{1} \\ & & 1 & c & d_{2} \\ & & 1 & \\ & & & 1\end{array}\right)$, and $Z_{U T_{5}\left(\mathbf{F}_{q}\right)}(A, B)=\left\{\left(\begin{array}{ccccc}1 & x_{1} & \lambda_{1} z_{3} & y_{2} & x_{2} \\ & 1 & & z_{2} & w_{1} \\ & & & z_{3} & \lambda_{2} z_{3}+\mu x_{1} \\ & & & \\ & & & & 1\end{array}\right)\right.$ $\left.\begin{array}{c}\lambda_{1}=\frac{b_{1}}{c}, \lambda_{2}=\frac{d_{2}}{c}, \\ \mu=\frac{d_{1}}{b_{1}}\end{array}\right\}$.

This is isomorphic to a centralizer of canonical form of type $A_{4}$. So $(A, B)$ is a branch of type $A_{4}$, and there are $q^{2}(q-1)^{2}$ such branches.

When $b_{1}=0$. Then we have $y_{1}=0$. Hence Equation 7.3 becomes $x_{1} d_{1}+a_{2}=a_{2}^{\prime}$. When $d_{1} \neq 0$, choose $x_{1}$ so that $a_{2}^{\prime}=0$. B is reduced to $\left(\begin{array}{lllll}1 & & & & \\ & 1 & & & d_{1} \\ & 1 & c & d_{2} \\ & & 1 & \\ & & & 1\end{array}\right)$, and

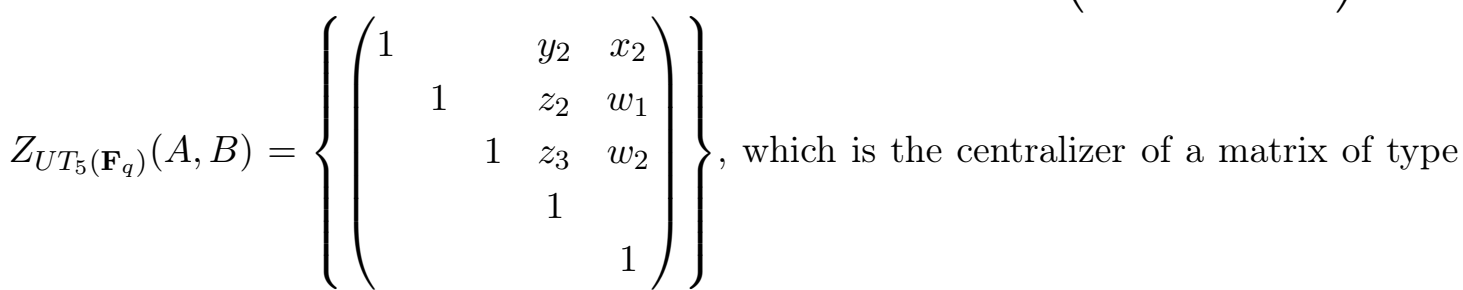
$R_{1}$. Thus $(A, B)$ is of type $R_{1}$, and thus there are $q(q-1)^{2}$ branches of this type.

When $d_{1}=0$, and $d_{2} \neq 0$. Equation 7.3 ends up becoming $a_{2}^{\prime}=a_{2}$, and $B$ is reduced

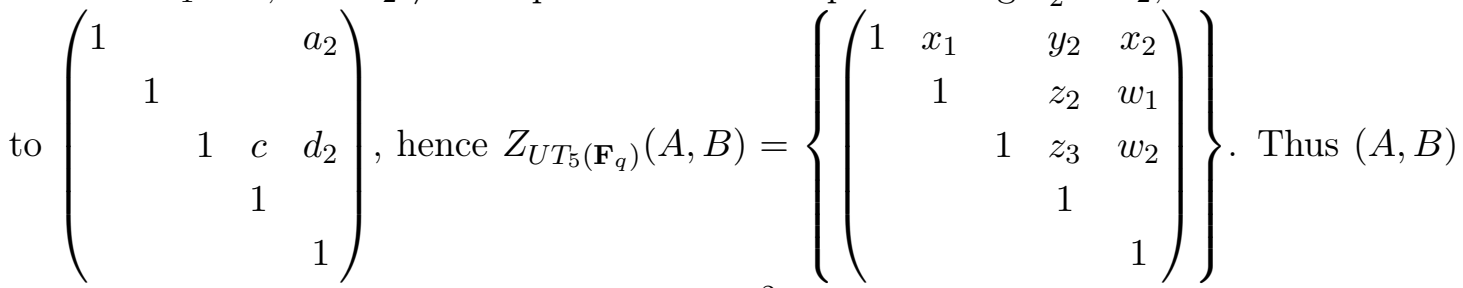
is a branch of type $A_{4}$, and there are $q(q-1)^{2}$ such branches.

When $C=\left(\begin{array}{ccc}1 & c_{1} & \\ & 1 & c_{2} \\ & & 1\end{array}\right), c \neq 0$ : Here $Z=\left(\begin{array}{ccc}1 & z_{1} & z_{2} \\ & 1 & \lambda_{0} z_{1} \\ & & 1\end{array}\right)$, where $\lambda_{0}=\frac{c_{2}}{c_{1}}$. Thus, from Equation 17.1, we have: $\left(\begin{array}{cc}c_{1} x_{1}+b_{1} & c_{2} y_{1}+b_{2}\end{array}\right)=\left(\begin{array}{cc}b_{1}^{\prime} & \lambda_{0} z_{1} b_{1}^{\prime}+b_{2}^{\prime}\end{array}\right)$. So, we choose $x_{1}$ so that $b_{1}^{\prime}=0$. Similarly, we choose $y_{1}$ such that $b_{2}^{\prime}=0$. Thus, on replacing $b_{1}$, and $b_{2}$ by 0 in the above equation, we get that $x_{1}=0$, and $y_{1}=0$. 
Equation 7.2 becomes $\left(\begin{array}{c}d_{1}+z_{1} d_{2} \\ d_{2} \\ 0\end{array}\right)=\left(\begin{array}{c}d_{1}^{\prime}+c_{1} w_{2} \\ d_{2}^{\prime} \\ 0\end{array}\right)$. Thus $d_{2}^{\prime}=d_{2}$, and we can choose $w_{2}$ so that $d_{1}^{\prime}=0$. So we are left with $d_{2} \neq 0$. With $x_{1}=y_{1}=b_{1}=0$, Equation 7.3 becomes $a_{2}^{\prime}=a_{2}$. Hence $B$ is reduced to $\left(\begin{array}{cccccc}1 & & & & a_{2} \\ & 1 & c_{1} & & \\ & 1 & c_{2} & d_{2} \\ & & 1 & \\ & & & & 1\end{array}\right)$, with $Z_{U T_{5}\left(\mathbf{F}_{q}\right)}(A, B)=\left\{\left(\begin{array}{ccccc}1 & & & y_{2} & x_{2} \\ & 1 & z_{1} & z_{2} & w_{1} \\ & & 1 & \lambda_{0} z_{1} & \lambda_{1} z_{1} \\ & & 1 & \\ & & & & 1\end{array}\right) \mid \lambda_{0}=\frac{c_{2}}{c_{1}}, \lambda_{1}=\frac{d_{2}}{c_{1}}\right\}$, which is a centralizer of type $R_{2}$. $(A, B)$ is a branch of type $R_{2}$, and there are $q(q-1)^{3}$ branches of this type.

Case $a_{1} \neq 0$ : We look at the various types of $C$ as our subcases.

When $C=I_{3}$ : Here Equation 7.1 becomes:

$$
\left(\begin{array}{lll}
a_{1} & b_{1} & b_{2}
\end{array}\right)=\left(\begin{array}{lll}
a_{1} & b_{1}^{\prime}+a_{1} z_{1} & b_{2}^{\prime}+b_{1}^{\prime} z_{3}+z_{2} a_{1}
\end{array}\right) .
$$

Using a suitable $z_{1}$, we can make $b_{1}^{\prime}=0$, and using a suitable $z_{2}$, we can make $b_{2}^{\prime}=0$. Thus, on replacing $b_{1}$ and $b_{2}^{\prime}$ by 0 in the above equation, we have $z_{1}=z_{2}=0$. Hence with this, Equation 7.2 becomes $\left(\begin{array}{c}d_{1} \\ d_{2} \\ 0\end{array}\right)=\left(\begin{array}{c}d_{1}^{\prime} \\ d_{2}^{\prime} \\ 0\end{array}\right)$. Equation 7.3 is reduced to $a_{2}+x_{1} d_{1}+y_{1} d_{2}=$ $a_{2}^{\prime}+a_{1} w_{1}$. So we choose $w_{1}$ such that $a_{2}^{\prime}=0$. Thus $B$ is reduced to $\left(\begin{array}{ccccc}1 & a_{1} & & & \\ & 1 & & & d_{1} \\ & & 1 & & d_{2} \\ & & 1 & \\ & & & & 1\end{array}\right)$, and $Z_{U T_{5}\left(\mathbf{F}_{q}\right)}=\left\{\left(\begin{array}{ccccc}1 & x_{1} & y_{1} & y_{2} & x_{2} \\ & 1 & & & \lambda x_{1}+\mu y_{1} \\ & & 1 & z_{3} & w_{2} \\ & & & 1 & \\ & & & & 1\end{array}\right\} \mid \lambda=\frac{d_{1}}{a_{1}}, \mu=\frac{d_{2}}{a_{1}}\right\}$, which is the centralizer of type $B_{4}$. $(A, B)$ is thus a branch of type $B_{4}$, and there are $q^{2}(q-1)$ such branches. 
When $C=\left(\begin{array}{ccc}1 & & c \\ & 1 & \\ & & 1\end{array}\right), c \neq 0$ : Equation 7.1 becomes:

$$
\left(\begin{array}{lll}
a_{1} & b_{1} & b_{2}+c x_{1}
\end{array}\right)=\left(\begin{array}{lll}
a_{1} & a_{1} z_{1}+b_{1}^{\prime} & a_{1} z_{2}+b_{1}^{\prime} z_{3}+b_{2}^{\prime}
\end{array}\right)
$$

Choose $z_{1}$ and $z_{2}$ such that $b_{1}^{\prime}=b_{2}^{\prime}=0$. Again, like in the previous case on replacing $b_{1}$ and $b_{2}$ by 0 in the above equation, we have $z_{1}=04$ and $z_{2}=\frac{c}{a_{1}} x_{1}$. From Equation 7.2 , we get $d_{1}^{\prime}=d_{1}$ and $d_{2}^{\prime}=d_{2}$. Equation 7.3 is reduced to $x_{1} d_{1}+y_{1} d_{2}+a_{2}=w_{1} a_{1}+$ $a_{2}^{\prime}$. We choose $w_{1}$ such that $a_{2}^{\prime}=0$. Thus $B$ is reduced to $\left(\begin{array}{ccccc}1 & a_{1} & & & \\ & 1 & & c & d_{1} \\ & & 1 & & d_{2} \\ & & 1 & \\ & & & & 1\end{array}\right)$, and $Z_{U T_{5}\left(\mathbf{F}_{q}\right)}(A, B)=\left\{\left(\begin{array}{ccccc}1 & x_{1} & y_{1} & y_{2} & x_{2} \\ & 1 & & \lambda_{1} x_{1} & \lambda_{2} x_{1}+\mu y_{1} \\ & & 1 & z_{3} & w_{2} \\ & & 1 & \\ & & & & 1\end{array}\right) \mid \lambda_{1}=\frac{c}{a_{1}}, \lambda_{2}=\frac{d_{1}}{a_{1}}, \mu=\frac{d_{2}}{a_{1}}\right\}$. This is of type $B_{4}$. Hence $(A, B)$ is a branch of type $B_{4}$, and there are $(q-1)^{2} q^{2}$ such branches.

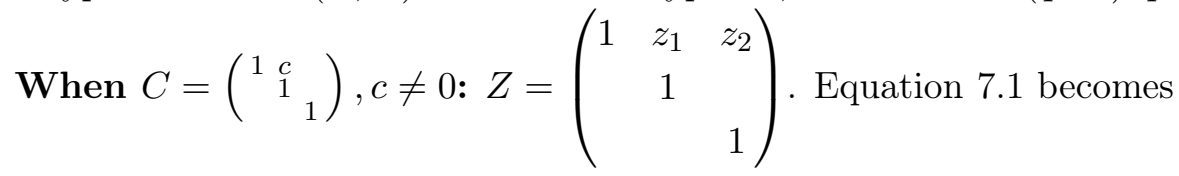

$$
\begin{aligned}
& \left(\begin{array}{lll}
a_{1} & c y_{1}+b_{1} & b_{2}
\end{array}\right)=\left(\begin{array}{lll}
a_{1} & a_{1} z_{1}+b_{1}^{\prime} & a_{1} z_{2}+b_{2}^{\prime}
\end{array}\right)
\end{aligned}
$$

Choose $z_{1}$ such that $b_{1}^{\prime}-0$, and choose $z_{2}$ such that $b_{2}^{\prime}=0$. So, on substituting $b_{1}$ and $b_{2}$ with 0 in the above, we have $z_{1}=\frac{c}{a_{1}} y_{1}$, and $z_{2}=0$. Thus Equation 7.2 is reduced to $\left(\begin{array}{c}d_{1}+\frac{c}{a_{1}} y_{1} d_{2} \\ d_{2} \\ 0\end{array}\right)=\left(\begin{array}{c}c w_{2}+d_{1}^{\prime} \\ d_{2}^{\prime} \\ 0\end{array}\right)$. We have $d_{2}^{\prime}=d_{2}$. Choose $w_{2}$ so that $d_{1}^{\prime}=0$. Equation 7.3 is reduced to $y_{1} d_{2}+a_{2}=a_{1} w_{1}+a_{2}^{\prime}$. Choose $w_{1}$ such that $a_{2}^{\prime}=0$. Thus $B$ is reduced to $\left(\begin{array}{ccccc}1 & a_{1} & & & \\ & 1 & c & & \\ & & 1 & & d_{2} \\ & & & 1 & \\ & & & & 1\end{array}\right)$, with $Z_{U T_{5}\left(\mathbf{F}_{q}\right)}(A, B)=\left\{\left(\begin{array}{ccccc}1 & x_{1} & y_{1} & y_{2} & x_{2} \\ & 1 & \frac{c}{a_{1}} y_{1} & & \frac{d_{2}}{a_{1}} y_{1} \\ & & & & \frac{d_{2}}{a_{1}} y_{1} \\ & & 1 & \\ & & & & 1\end{array}\right)\right\}$, which is a centralizer of a matrix of type $R_{3}$. So $(A, B)$ is a branch of type $R_{3}$, and there are $(q-1)^{2} q$ branches of this type. 


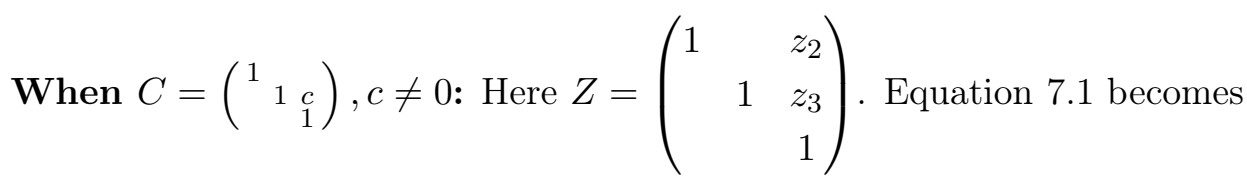

$$
\begin{aligned}
& \left(\begin{array}{lll}
a_{1} & b_{1} & b_{2}+c y_{2}
\end{array}\right)=\left(\begin{array}{lll}
a_{1} & b_{1}^{\prime} & a_{1} z_{2}+b_{1}^{\prime} z_{3}+b_{2}^{\prime}
\end{array}\right) .
\end{aligned}
$$

We have $b_{1}^{\prime}=b_{1}$. we can choose $y_{2}$ such that $b_{2}^{\prime}=0$. Thus, on replacing $b_{2}$ by 0 in the above equation, we have $y_{2}=\frac{a_{1}}{c} z_{2}+\frac{b_{1}}{c} z_{3}$. And Equation 7.2 ends up giving us $d_{1}^{\prime}=d_{1}$, and $d_{2}^{\prime}=d_{2}$. Thus Equation 7.3 stays as it is. Since $a_{1} \neq 0$, we choose $w_{1}$ so that $a_{2}^{\prime}=0 . \quad B$ is therefore reduced to $\left(\begin{array}{ccccc}1 & a_{1} & b_{1} & \\ & 1 & & & d_{1} \\ & 1 & c & d_{2} \\ & & 1 & \\ & & & 1\end{array}\right) . Z_{U T_{5}\left(\mathbf{F}_{q}\right)}(A, B)=$
$\left\{\left(\begin{array}{ccccc}1 & x_{1} & y_{1} & \frac{a_{1}}{c} z_{2}+\frac{b_{1}}{c} z_{3} & x_{2} \\ & 1 & & z_{2} & \frac{d_{1}}{a_{1}} x_{1}+\frac{d_{2}}{a_{1}} y_{1}-\frac{b_{1}}{a_{1}} w_{2} \\ & & & z_{3} & w_{2} \\ & & 1 & & \end{array}\right)\right\}$, which is that of type $B_{4} .(A, B)$ is of type $B_{4}$, and the number of branches is $q^{3}(q-1)^{2}$.

$$
\begin{gathered}
\text { When } C=\left(\begin{array}{ccc}
1 & c_{1} & \\
& 1 & c_{2} \\
& & 1
\end{array}\right), c_{1}, c_{2} \neq 0 \text { : Here } Z=\left(\begin{array}{ccc}
1 & z_{1} & z_{2} \\
& 1 & \frac{c_{2}}{c_{1}} z_{1} \\
& 1
\end{array}\right) \text {. Equation } 7.1 \text { becomes: } \\
\qquad\left(\begin{array}{lll}
a_{1} & b_{1}+c_{1} x_{1} & b_{2}+c_{2} y_{1}
\end{array}\right)=\left(\begin{array}{lll}
a_{1} & a_{1} z_{1}+b_{1}^{\prime} & a_{1} z_{2}+b_{1}^{\prime} \frac{c_{2}}{c_{1}} z_{1}+b_{2}^{\prime}
\end{array}\right) .
\end{gathered}
$$

Choose $z_{1}$ such that $b_{1}^{\prime}=0$, and choose $z_{2}$ such that $b_{2}=0$. On replacing $b_{1}$ and $b_{2}$ by 0 in the above equation, we see that $z_{1}=\frac{c_{1}}{a_{1}} x_{1}$, and $z_{2}=\frac{c_{2}}{a_{1}} y_{1}$. From Equation 7.2 , we have $d_{1}+\frac{c_{1}}{a_{1}} x_{1} d_{2}=c_{1} w_{2}+d_{1}^{\prime}$, and $d_{2}^{\prime}=d_{2}$. So we choose $w_{2}$ such that $d_{1}^{\prime}=0$. Equation 7.3 becomes: $y_{1} d_{2}+a_{2}=w_{1} a_{1}+a_{2}^{\prime}$. Choose $w_{1}$ such that $a_{2}^{\prime}=0$. Thus $B$ is reduced to $\left(\begin{array}{ccccc}1 & a_{1} & & & \\ & 1 & c_{1} & & \\ & & 1 & c_{2} & d_{2} \\ & & & 1 & \\ & & & & 1\end{array}\right)$, and $Z_{U T_{5}\left(\mathbf{F}_{q}\right)}=\left\{\left(\begin{array}{ccccc}1 & x_{1} & y_{1} & y_{2} & x_{2} \\ & 1 & \frac{c_{1}}{a_{1}} x_{1} & \frac{c_{2}}{a_{1}} y_{1} & \frac{d_{2}}{a_{1}} y_{1} \\ & & & \frac{c_{2}}{a_{1}} x_{1} & \frac{d_{2}}{a_{1}} x_{1} \\ & & 1 & \\ & & & 1\end{array}\right)\right\}$, which is the centralizer of a matrix of type $R_{3}$. Thus $(A, B)$ is branch of type $R_{3}$, and there are $q(q-1)^{3}$ such branches. Hence, adding up the branches of each type, we get the numbers as mentioned in the statement of this proposition.

Proposition 7.3. An upper unitriangular matrix of type $A_{2}$ has $q^{4}$ branches of type $A_{2}$, $2 q^{2}\left(q^{2}-1\right)$ branches of regular type $R_{1}$, and $q\left(q^{2}-1\right)^{2}$ branches of regular type $R_{2}$. 
Proof. Let $A=\left(\begin{array}{ccccc}1 & & & & \\ & 1 & & a & \\ & & 1 & & \\ & & 1 & \\ & & & 1\end{array}\right), a \neq 0$ a matrix of type $A_{2}$. The centralizer $Z_{U T_{5}}(A)$

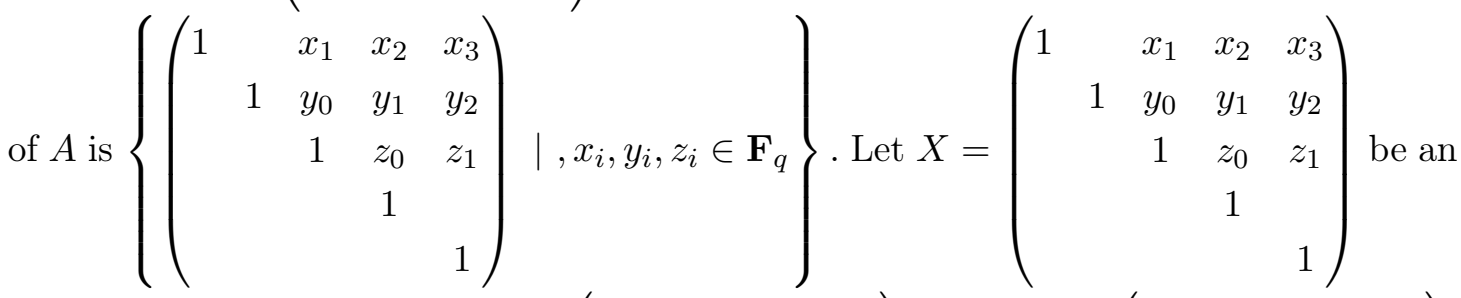

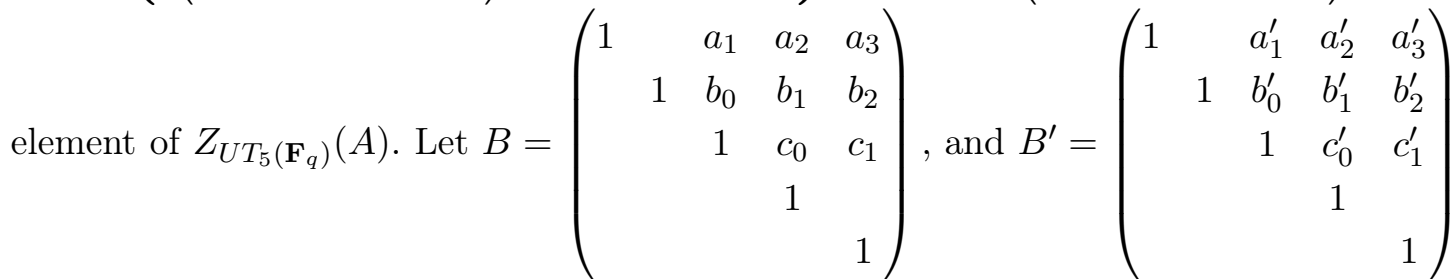
be a conjugate of $B$ by $X$. Thus equating $X B=B^{\prime} X$ gives us $a_{1}^{\prime}=a_{1}, b_{0}^{\prime}=b_{0}, c_{0}^{\prime}=c_{0}$, $c_{1}^{\prime}=c_{1}$, and the following equations:

$$
\begin{aligned}
& x_{1} c_{0}+a_{2}=a_{1} z_{0}+a_{2}^{\prime} \\
& x_{1} c_{1}+a_{3}=a_{1} z_{1}+a_{3}^{\prime} \\
& y_{0} c_{0}+b_{1}=b_{1}^{\prime}+b_{0}^{\prime} z_{0} \\
& y_{0} c_{1}+b_{2}=b_{2}^{\prime}+b_{0}^{\prime} z_{1}
\end{aligned}
$$

We consider two cases when $\left(a_{1}, b_{0}, c_{0}, c_{1}\right)=\mathbf{0}$ and when $\left(a_{1}, b_{0}, c_{0}, c_{1}\right) \neq \mathbf{0}$.

Case: $\left(a_{1}, b_{0}, c_{0}, c_{1}\right)=\mathbf{0}$. In this case, we get $a_{2}=a_{2}^{\prime}, a_{3}=a_{3}^{\prime}, b_{1}=b_{1}^{\prime}$ and $b_{2}=b_{2}^{\prime}$. Therefore $Z_{U T_{5}\left(\mathbf{F}_{q}\right)}(A, B)=Z_{U T_{5}\left(\mathbf{F}_{q}\right)}(A)$. So $(A, B)$ is a branch of type $A_{2}$, and there are $q^{4}$ branches.

Case: $\left(a_{1}, b_{0}, c_{0}, c_{1}\right) \neq \mathbf{0}$. First we consider that $c_{1} \neq 0$. We choose $x_{1}$ and $y_{0}$ in such a way that we get $a_{3}=b_{2}=0$. Now if $\left(a_{1}, b_{0}\right)=(0,0)$, then by simple calculations, we get $Z_{U T_{5}\left(\mathbf{F}_{q}\right)}(A, B)$ is a commutative group of size $q^{6}$. Thus $(A, B)$ is of regular type $R_{1}$, and there are $q^{3}(q-1)$ branches of this type. If we consider that case when at least one of $a_{1}$ and $b_{0}$ is non-zero, then we can choose $z_{0}$ suitably so that we get one of $a_{2}$ or $b_{1}$ equal to zero. By routine check, we get that $Z_{U T_{5}\left(\mathbf{F}_{q}\right)}(A, B)$ is a commutative group of size $q^{5}$. Thus $(A, B)$ is of regular type $R_{2}$, and there are $\left(q^{3}-q^{2}\right)\left(q^{2}-1\right)$ branches of this type.

Now we consider that $c_{1}=0$ and $c_{0} \neq 0$. We choose $x_{1}$ and $y_{0}$ in such a way that we get $a_{2}=b_{1}=0$. Now if $\left(a_{1}, b_{0}\right)=0$, then by simple calculations, we get $Z_{U T_{5}\left(\mathbf{F}_{q}\right)}(A, B)$ is a commutative group of size $q^{6}$. Thus $(A, B)$ is of regular type $R_{1}$, and there are $q^{2}(q-1)$ branches of this type. If we consider that case when at least one of $a_{1}$ and $b_{0}$ is non-zero, 
then we can choose $z_{1}$ suitably so that we get one of $a_{3}$ or $b_{2}$ equal to zero. By routine check, we get that $Z_{U T_{5}\left(\mathbf{F}_{q}\right)}(A, B)$ is a commutative group of size $q^{5}$. Thus $(A, B)$ is of regular type $R_{2}$, and there are $\left(q^{2}-q\right)\left(q^{2}-1\right)$ branches of this type.

Next we consider when $c_{1}=c_{0}=0$ and $b_{0} \neq 0$. We choose $z_{0}$ and $z_{1}$ in such a way that we get $b_{1}=b_{2}=0$. Now by simple calculations, we get $Z_{U T_{5}\left(\mathbf{F}_{q}\right)}(A, B)$ is a commutative group of size $q^{6}$. Thus $(A, B)$ is of regular type $R_{1}$, and there are $q^{3}(q-1)$ branches of this type.

Finaly we consider when $c_{1}=c_{0}=b_{0} 0$ and $a_{1} \neq 0$. We choose $z_{0}$ and $z_{1}$ in such a way that we get $a_{2}=a_{3}=0$. Now by simple calculations, we get $Z_{U T_{5}\left(\mathbf{F}_{q}\right)}(A, B)$ is a commutative group of size $q^{6}$. Thus $(A, B)$ is of regular type $R_{1}$, and there are $q^{2}(q-1)$ branches of this type.

Therefore a matrix of type $A_{2}$ has $q^{4}$ branches of type $A_{2}, 2 q^{2}\left(q^{2}-1\right)$ branches of regular type $R_{1}$, and $q\left(q^{2}-1\right)^{2}$ braches of regular type $R_{2}$.

Proposition 7.4. An upper unitriangular matrix of type $A_{3}$ has

\begin{tabular}{c|c||c|c}
\hline Branch & No. of Branches & Branch & No. of Branches \\
\hline$A_{3}$ & $q^{2}$ & $R_{1}$ & $q\left(q^{2}+q-1\right)(q-1)$ \\
$A_{4}$ & $q\left(q^{2}-1\right)$ & $R_{2}$ & $q\left(q^{2}-1\right)(q-1)$ \\
$B_{3}$ & $q^{2}(q-1)$ & $R_{3}$ & $q^{2}(q-1)^{2}$ \\
$B_{5}$ & $q^{3}(q-1)$ & $U N T_{1}$ & $q^{2}(q-1)$. \\
\hline
\end{tabular}

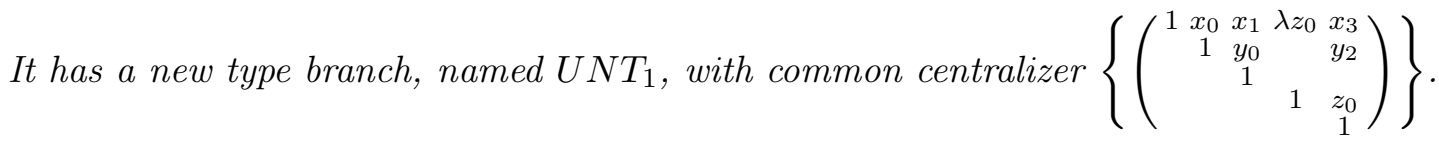
Proof. Let $A=\left(\begin{array}{ccccc}1 & & a & & \\ & 1 & & & \\ & & 1 & & \\ & & & 1 & \\ & & & & 1\end{array}\right), a \neq 0$ a matrix of type $A_{3}$. The centralizer $Z_{U T_{5}}(A)$ of $A$ is $\left\{\left(\begin{array}{ccccc}1 & x_{0} & x_{1} & x_{2} & x_{3} \\ & 1 & y_{0} & y_{1} & y_{2} \\ & & 1 & & \\ & & & 1 & z_{0} \\ & & & & 1\end{array}\right) \mid, x_{i}, y_{i}, w_{0} \in \mathbf{F}_{q}\right\}$. Let $X=\left(\begin{array}{ccccc}1 & x_{0} & x_{1} & x_{2} & x_{3} \\ & 1 & y_{0} & y_{1} & y_{2} \\ & 1 & & \\ & & 1 & w_{0} \\ & & & & 1\end{array}\right)$ be an element of $Z_{U T_{5}\left(\mathbf{F}_{q}\right)}(A)$. Let $B=\left(\begin{array}{ccccc}1 & a_{0} & a_{1} & a_{2} & a_{3} \\ & 1 & b_{0} & b_{1} & b_{2} \\ & & 1 & & \\ & & & 1 & d_{0} \\ & & & & 1\end{array}\right)$, and $B^{\prime}=\left(\begin{array}{ccccc}1 & a_{0}^{\prime} & a_{1}^{\prime} & a_{2}^{\prime} & a_{3}^{\prime} \\ & 1 & b_{0}^{\prime} & b_{1}^{\prime} & b_{2}^{\prime} \\ & & 1 & & \\ & & & 1 & d_{0}^{\prime} \\ & & & & 1\end{array}\right)$ 
be a conjugate of $B$ by $X$. Thus equating $X B=B^{\prime} X$ gives us $a_{0}^{\prime}=a_{0}, b_{0}^{\prime}=b_{0}, b_{1}^{\prime}=b_{1}$, $d_{0}^{\prime}=d_{0}$, and the following equations:

$$
\begin{aligned}
a_{1}+x_{0} b_{0} & =a_{0} y_{0}+a_{1}^{\prime} \\
a_{2}+x_{0} b_{1} & =a_{0} y_{1}+a_{2}^{\prime} \\
a_{3}+x_{0} b_{2}+x_{2} d_{0} & =a_{0} y_{2}+a_{2}^{\prime} z_{0}+a_{3}^{\prime} \\
b_{2}+y_{1} d_{0} & =z_{0} b_{1}+b_{2}^{\prime}
\end{aligned}
$$

We consider two cases when $\left(a_{0}, b_{0}, b_{1}, d_{0}\right)=\mathbf{0}$ and when $\left(a_{0}, b_{0}, b_{1}, d_{0}\right) \neq \mathbf{0}$.

Case: $\left(a_{0}, b_{0}, b_{1}, d_{0}\right)=\mathbf{0}$. In this case, we get $a_{1}^{\prime}=a_{1}, a_{2}^{\prime}=a_{2}, b_{2}^{\prime}=b_{2}$, and $a_{3}+x_{0} b_{2}=a_{2} z_{0}+a_{3}^{\prime}$.

If $\left(a_{2}, b_{2}\right)=\mathbf{0}$, then we get $b_{3}=b_{3}^{\prime}$. Therefore $Z_{U T_{5}\left(\mathbf{F}_{q}\right)}(A, B)=Z_{U T_{5}\left(\mathbf{F}_{q}\right)}(A)$. So $(A, B)$ is a branch of type $A_{3}$, and there are $q^{2}$ branches. Now we consider that $a_{2} \neq 0$. In this case, we can choose $w_{0}$ in such a way that we get $a_{3}=0$. By routine check, we get $Z_{U T_{5}\left(\mathbf{F}_{q}\right)}(A, B)$ is a group of order $q^{7}$ and $(A, B)$ is the type $B_{3}$, and there are $q^{2}(q-1)$ branches.

If we consider $a_{2}=0$ and $b_{2} \neq 0$, choose $x_{0}$ in such a way that we get $a_{3}=0$. By routine check, we get $Z_{U T_{5}\left(\mathbf{F}_{q}\right)}(A, B)$ is a group of order $q^{7}$ and $(A, B)$ is a branch of type $A_{4}$, and there are $q(q-1)$ branches.

Case: $\left(a_{0}, b_{0}, b_{1}, d_{0}\right) \neq \mathbf{0}$. First we consider that $a_{0} \neq 0$. In this case, we can choose $y_{0}, y_{1}$ and $y_{2}$ in such a way that we get $a_{1}=a_{2}=a_{3}=0$ and $b_{2}+\frac{d_{0} b_{1}}{b_{0}} x_{0}=z_{0} b_{1}+b_{2}^{\prime}$. Now if $b_{1}=0$, then we get $b_{2}=b_{2}^{\prime}$. By routine check, we get $Z_{U T_{5}\left(\mathbf{F}_{q}\right)}(A, B)$ is a group of order $q^{5}$ and $(A, B)$ is a branch of type $B_{5}$, and there are $q^{3}(q-1)$ branches. On the other hand if $b_{1} \neq 0$, then we choose $z_{0}$ in such a way that we get $b_{2}=0$ By routine check, we get $Z_{U T_{5}\left(\mathbf{F}_{q}\right)}(A, B)$ is a commutative group of order $q^{4}$ and $(A, B)$ is a branch of regular type $R_{3}$, and there are $q^{2}(q-1)^{2}$ branches.

Now we consider that $a_{0}=0$ and $b_{0} \neq 0$. In this case, we can choose $x_{0}$ in such a way that we get $a_{1}=0$ and this implies $x_{0}=0$. Thus we get $b_{2}=b_{2}^{\prime}$ and the following equalities: $\begin{aligned} a_{3}+x_{2} d_{0} & =a_{2} z_{0}+a_{3}^{\prime} \\ b_{2}+d_{0} y_{1} & =z_{0} b_{1}+b_{2}^{\prime}\end{aligned} \quad$ Now if $\left(d_{0}, a_{2}, b_{1}\right)=\mathbf{0}$, then we get $a_{3}=a_{3}^{\prime}$ and $b_{2}=b_{2}^{\prime}$. By routine check, we get $Z_{U T_{5}\left(\mathbf{F}_{q}\right)}(A, B)$ is a group of order $q^{7}$ and $(A, B)$ is a branch of type $A_{4}$, and there are $q^{2}(q-1)$ branches. If $d_{0} \neq 0$, then we choose $x_{2}$ and $y_{1}$ in such a way that we get $a_{3}=b_{2}=0$. By routine check, we get $Z_{U T_{5}\left(\mathbf{F}_{q}\right)}(A, B)$ is a commutative group of order $q^{5}$ and $(A, B)$ is a branch of regular type $R_{2}$, and there are $q^{2}(q-1)^{2}$ branches.

If $d_{0}=0$ and $a_{2} \neq 0$, then we choose $w_{0}$ in such a way that we get $a_{3}=0$ and this implies $w_{0}=0$. Thus we get $b_{2}=b_{2}^{\prime}$. By routine check, we get $Z_{U T_{5}\left(\mathbf{F}_{q}\right)}(A, B)$ is a commutative group of order $q^{6}$ and $(A, B)$ is a branch of regular type $R_{1}$, and there are $q^{2}(q-1)^{2}$ branches. 
If $d_{0}=a_{2}=0$ and $b_{1} \neq 0$, then we get $a_{3}=a_{3}^{\prime}$ and we choose $w_{0}$ in such a way that we get $b_{2}=0$. By routine check, we get $Z_{U T_{5}\left(\mathbf{F}_{q}\right)}(A, B)$ is a commutative group of order $q^{6}$ and $(A, B)$ is a branch of regular type $R_{1}$, and there are $q(q-1)^{2}$ branches.

Now we consider that $a_{0}=b_{0}=0$ and $b_{1} \neq 0$. In this case, we can choose $x_{0}$ and $z_{0}$ in such a way that we get $a_{2}=b_{2}=0$. In addition to this, if $d_{0}=0$, then we get $a_{3}=a_{3}^{\prime}$. By routine check, we get $Z_{U T_{5}\left(\mathbf{F}_{q}\right)}(A, B)$ is a commutative group of order $q^{6}$ and $(A, B)$ is a branch of regular type $R_{3}$, and there are $q^{2}(q-1)$ branches. Now if we consider $d_{0} \neq 0$, then we can choose $x_{2}$ in such a way that we get $a_{3}=0$. By routine check, we get $Z_{U T_{5}\left(\mathbf{F}_{q}\right)}(A, B)$ is a commutative group of order $q^{5}$ and $(A, B)$ is a branch of regular type $R_{2}$, and there are $q(q-1)^{2}$ branches.

Finally we consider the case when $a_{0}=b_{0}=b_{1}=0$ and $d_{0} \neq 0$, then we get $a_{2}=$ $a_{2}^{\prime}, a_{1}=a_{1}^{\prime}$ and we can choose $y_{1}$ and $x_{2}$ in such a way that we get $a_{3}=b_{2}=0$. By routine check, we get $Z_{U T_{5}\left(\mathbf{F}_{q}\right)}(A, B)$ is a group of order $q^{6}$, and $Z_{U T_{5}\left(\mathbf{F}_{q}\right)}(A, B)=$ $\left\{\left(\begin{array}{ccccc}1 & x_{0} & x_{1} & \lambda z_{0} & x_{3} \\ & 1 & y_{0} & & y_{2} \\ & & 1 & 1 & z_{0} \\ & & 1 & z_{0}\end{array}\right)\right\}$. As we have not seen this centralizer before, and This $(A, B)$ is a branch of new type, which we call $U N T_{1}$ and there are $q^{2}(q-1)$ branches.

Proposition 7.5. An upper unitriangular matrix of type $A_{4}$ has $q^{4}$ branches of type $A_{4}$, $q^{3}\left(q^{2}-1\right)$ branches of regular type $R_{1}$, and $q^{4}(q-1)$ breaches of regular type $R_{2}$.

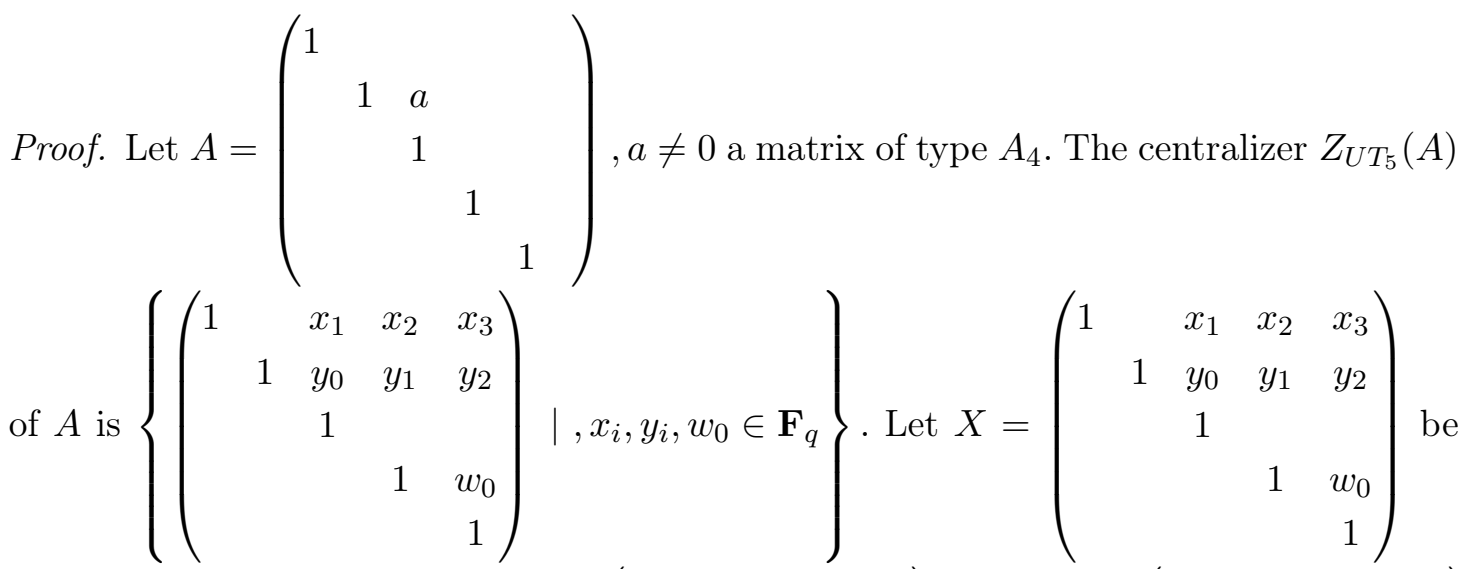

an element of $Z_{U T_{5}\left(\mathbf{F}_{q}\right)}(A)$. Let $B=\left(\begin{array}{ccccc}1 & & a_{1} & a_{2} & a_{3} \\ & 1 & b_{0} & b_{1} & b_{2} \\ & & 1 & & \\ & & & 1 & d_{0} \\ & & & & 1\end{array}\right)$, and $B^{\prime}=\left(\begin{array}{ccccc}1 & & a_{1}^{\prime} & a_{2}^{\prime} & a_{3}^{\prime} \\ & 1 & b_{0}^{\prime} & b_{1}^{\prime} & b_{2}^{\prime} \\ & 1 & & \\ & & & 1 & d_{0}^{\prime} \\ & & & & 1\end{array}\right)$

be a conjugate of $B$ by $X$. Thus equating $X B=B^{\prime} X$ gives us $a_{1}=a_{1}^{\prime} a,_{2}=a_{2}^{\prime}, b_{0}=b_{0}^{\prime}$, $b_{1}=b_{1}^{\prime}, d_{0}=d_{0}^{\prime}$ and the following equations:

$$
\begin{gathered}
a_{3}+x_{2} d_{0}=a_{2}^{\prime} w_{0}+a_{3}^{\prime} \\
b_{2}+d_{0} y_{1}=w_{0} b_{1}^{\prime}+b_{2}^{\prime} \\
71
\end{gathered}
$$


We consider two cases when $\left(a_{2}, b_{1}, d_{0}\right)=\mathbf{0}$ and when $\left(a_{2}, b_{1}, d_{0}\right) \neq \mathbf{0}$.

Case: $\left(a_{2}, b_{1}, d_{0}\right)=\mathbf{0}$. In this case, we get $a_{3}=a_{3}^{\prime}$ and $b_{2}=b_{2}^{\prime}$. Therefore $Z_{U T_{5}\left(\mathbf{F}_{q}\right)}(A, B)=Z_{U T_{5}\left(\mathbf{F}_{q}\right)}(A)$. So $(A, B)$ is a branch of type $A_{4}$, and there are $q^{4}$ branches.

Case: $\left(a_{2}, b_{1}, d_{0}\right) \neq \mathbf{0}$. First we consider that $d_{0} \neq 0$. Now we can choose $x_{2}$ and $y_{1}$ in such a way that we get $a_{3}=b_{2}=0$. By routine check, we get $Z_{U T_{5}\left(\mathbf{F}_{q}\right)}(A, B)$ is a commutative group of size $q^{5}$. Thus $(A, B)$ is of regular type $R_{2}$, and there are $q^{4}(q-1)$ branches of this type.

Now we consider that $d_{0}=0$ and $a_{2} \neq 0$. In this case, we can choose $w_{0}$ in such a way that we get $a_{3}=0$. By routine check, we get $Z_{U T_{5}\left(\mathbf{F}_{q}\right)}(A, B)$ is a commutative group of size $q^{6}$. Thus $(A, B)$ is of regular type $R_{1}$, and there are $q^{4}(q-1)$ branches of this type.

Finaly we consider when $d_{0}=a_{2}=0$ and $b_{1} \neq 0$, now we can choose $w_{0}$ in such a way that we get $b_{2}=0$. Again, we get $Z_{U T_{5}\left(\mathbf{F}_{q}\right)}(A, B)$ is commutative group of size $q^{6}$. Thus $(A, B)$ is of regular type $R_{1}$, and there are $q^{3}(q-1)$ branches of this type.

Therefore we get that a matrix of type $A_{4}$ has $q^{4}$ branches of type $A_{4}, q^{3}\left(q^{2}-1\right)$ braches of regular type $R_{1}$, and $q^{4}(q-1)$ braches of regular type $R_{2}$.

Proposition 7.6. An upper unitriangular matrix of type $A_{5}$ has:

\begin{tabular}{c|c||c|c}
\hline Branch Type & No. of Branches & Branch Type & No. of Branches \\
\hline$A_{5}$ & $q^{2}$ & $R_{2}$ & $q^{2}(q-1)$ \\
$B_{4}$ & $2 q(q-1)$ & $R_{3}$ & $q(q-1)\left(q^{2}-1\right)$ \\
$B_{6}$ & $q^{2}\left(q^{2}-1\right)$ & $U N T_{1}$ & $q(q-1)^{2}$. \\
\hline
\end{tabular}

It has the new branch $U N T_{1}$ already seen in previous case.

Proof. There are several canonical forms for a matrix in $U T_{5}\left(\mathbf{F}_{q}\right)$, of type $A_{5}$. We prove this proposition for the canonical form $A=\left(\begin{array}{cccc}1 & a & & \\ & 1 & & \\ & & & \\ & & & \\ & & 1 & \\ & & & 1\end{array}\right)$, where $a \neq 0$. We have: $Z_{U_{5}\left(\mathbf{F}_{q}\right)}(A)=\left\{\left(\begin{array}{ccccc}1 & a_{0} & a_{1} & a_{2} & a_{3} \\ & 1 & & & \\ & & 1 & b_{0} & b_{1} \\ & & & 1 & c_{0} \\ & & & & 1\end{array}\right)\right\}$. We can rewrite this centralizer subgroup as

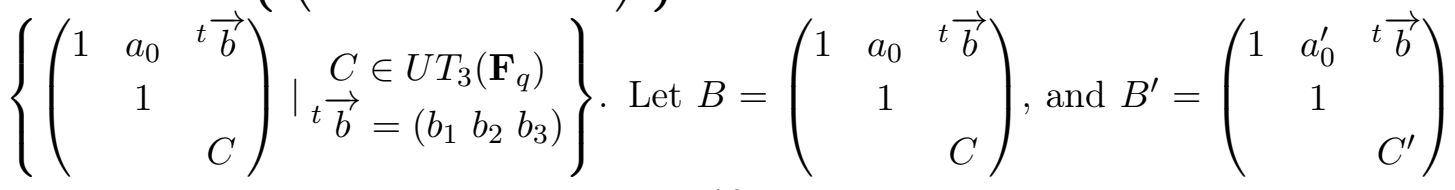


be a conjugate in $U T_{5}$ of $B . B^{\prime}=X B X^{-1}$, where $X=\left(\begin{array}{ccc}1 & x_{0} & { }^{t} \vec{y} \\ & 1 & \\ & & Z\end{array}\right)$. So, equating $X B=B^{\prime} X$ gives us $a_{0}^{\prime}=a_{0}, Z C=C^{\prime} Z$. So, we may take $C$ to be the representative of a conjugacy class in $U T_{3}\left(\mathbf{F}_{q}\right)$, and we have the equation:

$$
{ }^{t} \vec{y} \cdot C+{ }^{t} \vec{b}={ }^{t} \overrightarrow{b^{\prime}} Z+{ }^{t} \vec{y}
$$

We rewrite this equation slightly to get:

$$
\left(\begin{array}{lll}
y_{1} & y_{2} & y_{3}
\end{array}\right)\left(C-I_{3}\right)+\left(\begin{array}{lll}
b_{1} & b_{2} & b_{3}
\end{array}\right)=\left(\begin{array}{lll}
b_{1}^{\prime} & b_{2}^{\prime} & b_{3}^{\prime}
\end{array}\right) Z
$$

The cases:

When $C=I_{3}$. Here Equation 7.5 becomes: $\left(\begin{array}{lll}b_{1} & b_{2} & b_{3}\end{array}\right)=\left(\begin{array}{lll}b_{1}^{\prime} & b_{2} & b_{3}^{\prime}\end{array}\right)\left(\begin{array}{ccc}1 & z_{0} & z_{1} \\ & 1 & z_{2} \\ & & 1\end{array}\right)$, which gives us $b_{1}^{\prime}=b_{1}$, and the following equation:

$$
\begin{aligned}
& b_{2}=b_{2}^{\prime}+z_{0} b_{1} \\
& b_{3}=b_{3}^{\prime}+z_{1} b_{1}+z_{2} b_{2}^{\prime}
\end{aligned}
$$

We have two subcases here: When $b_{1}=0$ and when $b_{1} \neq 0$.

When $b_{1}=0$ Equation 7.6 becomes $b_{2}^{\prime}=b_{2}$, and Equation 7.7 becomes $b_{3}=b_{3}^{\prime}+z_{2} b_{2}$.

When $b_{2}=0$, we have $b_{3}^{\prime}=b_{3}$. So $B$ is reduced to $\left(\begin{array}{ccccc}1 & a_{0} & & & b_{3} \\ & 1 & & & \\ & & & & \\ & & 1 & \\ & & & 1\end{array}\right)$, and $Z_{U_{5}\left(\mathbf{F}_{q}\right)}(A, B)=Z_{U_{5}\left(\mathbf{F}_{q}\right)}(A)$. Thus $(A, B)$ is of type $A_{5}$, and there are $q^{2}$ such branches. When $b_{2} \neq 0$, in Equation [7.6, choose $z_{2}$ so that $b_{3}^{\prime}=0$. So, We have $B$ reduced to $\left(\begin{array}{ccccc}1 & a_{0} & & b_{2} & \\ & 1 & & & \\ & & 1 & & \\ & & & 1 & \\ & & & & 1\end{array}\right)$, and $Z_{U_{5}\left(\mathbf{F}_{q}\right)}(A, B)=\left\{\left(\begin{array}{ccccc}1 & x_{0} & y_{1} & y_{2} & y_{3} \\ & 1 & & & \\ & & 1 & z_{0} & z_{1} \\ & & & 1 & \\ & & & & 1\end{array}\right)\right\} .(A, B)$ is of type $B_{4}$ and there are $q(q-1)$ such branches.

When $b_{1} \neq 0$ : In Equation [7.6, choose $z_{0}$ such that $b_{2}^{\prime}=0$, and in Equation 7.6. choose $z_{1}$ such that $b_{3}^{\prime}=0$. So $B$ is reduced to $\left(\begin{array}{ccccc}1 & a_{0} & b_{1} & & \\ & 1 & & & \\ & & 1 & & \\ & & & 1 & \\ & & & & 1\end{array}\right)$, and $Z_{U_{5}\left(\mathbf{F}_{q}\right)}(A, B)=$ 


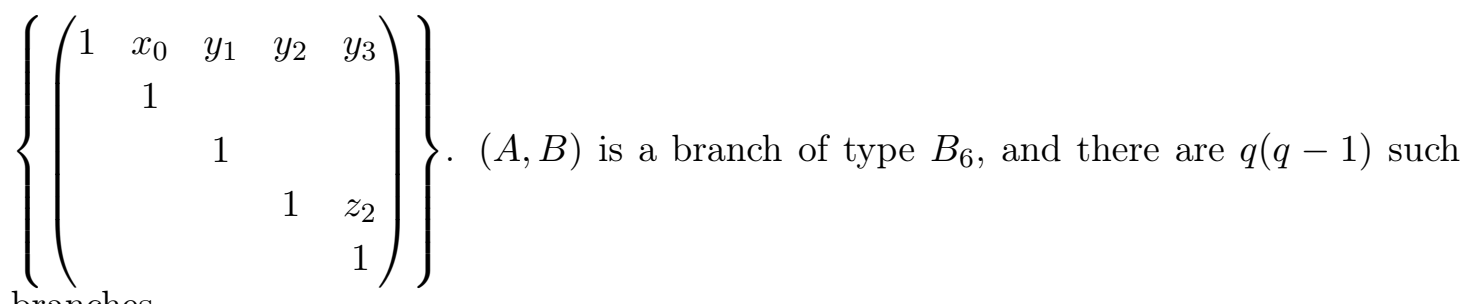
branches.

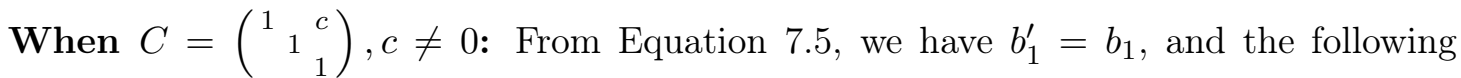
following equations:

$$
\begin{aligned}
b_{2} & =b_{2}^{\prime}+z_{0} b_{1} \\
b_{3}^{\prime}+c y_{1} & =b_{3}^{\prime}+z_{1} b_{1}^{\prime}+z_{2} b_{2}^{\prime} .
\end{aligned}
$$

As $c \neq 0$, choose $y_{1}$ so that $b_{3}^{\prime}=0$.

Case: $b_{1}=0$

We have $b_{2}^{\prime}=b_{2}$. When $b_{2}=0, B$ is reduced to $\left(\begin{array}{ccccc}1 & a_{0} & & & \\ & 1 & & & \\ & & 1 & & c \\ & & & 1 & \\ & & & & 1\end{array}\right)$, and $Z_{U_{5}\left(\mathbf{F}_{q}\right)}(A, B)=$ $\left\{\left(\begin{array}{ccccc}1 & x_{0} & & y_{2} & y_{3} \\ & 1 & & & \\ & & 1 & z_{0} & z_{1} \\ & & & 1 & z_{2} \\ & & & & 1\end{array}\right)\right\} .(A, B)$ is of type $B_{4}$, and there are $q(q-1)$ such branches.

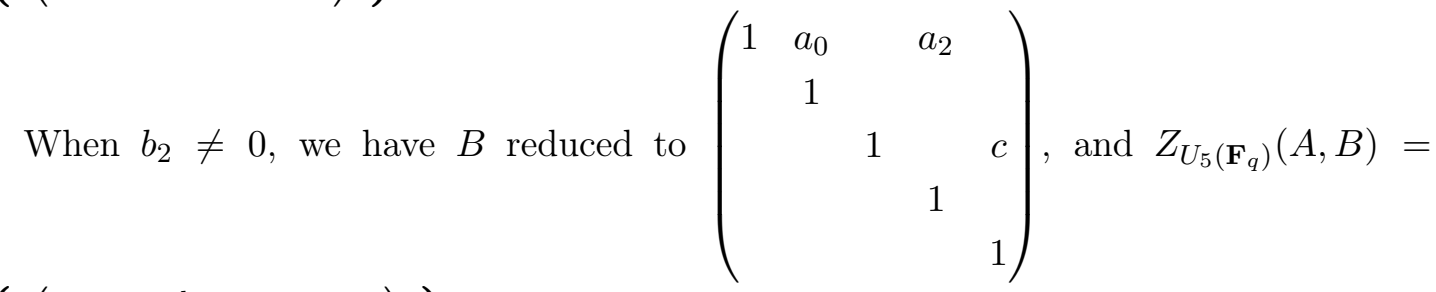
$\left\{\left(\begin{array}{ccccc}1 & x_{0} & \frac{b_{2}}{c} z_{2} & y_{2} & y_{3} \\ & 1 & & & \\ & & 1 & z_{0} & z_{1} \\ & & & 1 & z_{2} \\ & & & & 1\end{array}\right)\right\}$. This centralizer is isomorphic to that of a new type, $U N T_{1}$, which we had come across earlier. There are $q(q-1)^{2}$ such branches. 
When $b_{1} \neq 0$. We can choose $z_{0}$ so that $b_{2}^{\prime}=0$. Here $B$ is reduced to $\left(\begin{array}{ccccc}1 & a_{0} & a_{1} & & \\ & 1 & & & \\ & & & & c \\ & & & 1 & \\ & & & & 1\end{array}\right)$, and $Z_{U_{5}\left(\mathbf{F}_{q}\right)}(A, B)=\left\{\left(\begin{array}{ccccc}1 & x_{0} & \frac{b_{1}}{c_{1}} z_{1} & y_{2} & y_{3} \\ & 1 & & & \\ & & 1 & & z_{1} \\ & & & 1 & z_{2} \\ & & & & 1\end{array}\right)\right\}$. Hence $(A, B)$ is of type $B_{6}$, and there are $q(q-1)^{2}$ such branches.

When $C=\left(\begin{array}{ccc}1 & c & \\ & 1 & \\ & & 1\end{array}\right), c \neq 0$ : Here $Z=\left(\begin{array}{ccc}1 & z_{0} & z_{1} \\ & 1 & \\ & & 1\end{array}\right)$. With this, Equation 7.5 becomes: $\left(\begin{array}{lll}b_{1} & b_{2}+c y_{1} & b_{3}\end{array}\right)=\left(\begin{array}{lll}b_{1}^{\prime} & z_{0} b_{1}^{\prime}+b_{2}^{\prime} & z_{1} b_{1}^{\prime}+b_{3}^{\prime}\end{array}\right)$. Now, as $c \neq 0$, choose $y_{1}$ so that $b_{2}^{\prime}=0$.

When $b_{1}=0$, we have $b_{3}^{\prime}=b_{3}$. Thus, $B$ is reduced to $\left(\begin{array}{ccccc}1 & a_{0} & & & b_{3} \\ & 1 & & & \\ & & c & \\ & & 1 & \\ & & & 1\end{array}\right)$, and $Z_{U_{5}\left(\mathbf{F}_{q}\right)}(A, B)=\left\{\left(\begin{array}{ccccc}1 & x_{0} & & y_{2} & y_{3} \\ & 1 & & & \\ & & 1 & z_{0} & z_{1} \\ & & & 1 & \\ & & & & 1\end{array}\right)\right\}$. By a routine check, we can see that this centralizer is commutative, and of size $q^{5}$. $(A, B)$ is of type $R_{2}$, and there are $q^{2}(q-1)$ such branches. 
When $b_{1} \neq 0$, choose $z_{1}$ such that $b_{3}^{\prime}=0$. Thus, $B$ is reduced to $\left(\begin{array}{ccccc}1 & a_{0} & a_{1} & & \\ & 1 & & & \\ & & 1 & c & \\ & & & 1 & \\ & & & & 1\end{array}\right)$, and $Z_{U_{5}\left(\mathbf{F}_{q}\right)}(A, B)=\left\{\left(\begin{array}{ccccc}1 & x_{0} & \frac{b_{1}}{c} z_{0} & y_{2} & y_{3} \\ & 1 & & & \\ & & 1 & z_{0} & \\ & & & 1 & \\ & & & & 1\end{array}\right)\right\}$. This centralizer is of size $q^{4}$, and is commutative. Thus $(A, B)$ is of type $R_{3}$, and there are $(q-1)^{2} q$ such branches.

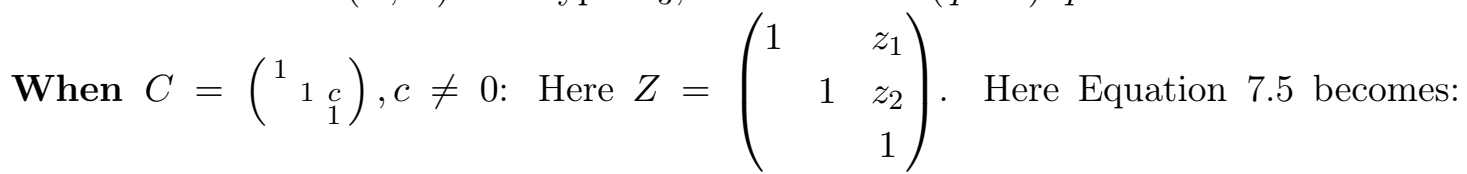
$\left(\begin{array}{lll}b_{1} & b_{2} & b_{3}+c y_{2}\end{array}\right)=\left(\begin{array}{lll}b_{1}^{\prime} & b_{2}^{\prime} & b_{3}^{\prime}+z_{1} b_{1}^{\prime}+z_{2} b_{2}^{\prime}\end{array}\right)$.

We have $b_{1}^{\prime}=b_{1}$ and $b_{2}^{\prime}=b_{2}$, and choose $y_{2}$ so that $b_{3}^{\prime}=0$. Thus $B$ is reduced to $\left(\begin{array}{ccccc}1 & a_{0} & b_{1} & b_{2} & \\ & 1 & & & \\ & & 1 & & \\ & & & 1 & c \\ & & & & 1\end{array}\right)$, and $Z_{U_{5}\left(\mathbf{F}_{q}\right)}(A, B)=\left\{\left(\left(\begin{array}{ccccc}1 & x_{0} & y_{1} & \frac{b_{1}}{c} z_{1}+\frac{b_{2}}{c} z_{2} & y_{3} \\ & 1 & & & \\ & 1 & & z_{1} \\ & & 1 & z_{2} \\ & & & 1\end{array}\right)\right\}\right.$. This too is of type $B_{6}$, and there are $q^{3}(q-1)$ such branches.

$$
\text { And now we have the last case: When } C=\left(\begin{array}{ccc}
1 & c & \\
& 1 & d \\
& & 1
\end{array}\right), c, d \neq 0 \text { : Here } Z=\left(\begin{array}{ccc}
1 & z_{0} & z_{1} \\
& 1 & \lambda z_{0} \\
& & 1
\end{array}\right) \text {, }
$$

where $\lambda=\frac{d}{c}$. Equation 7.5 becomes: $\left(\begin{array}{lll}b_{1} & b_{2}+c y_{1} & b_{3}+d y_{2}\end{array}\right)=\left(\begin{array}{lll}b_{1}^{\prime} & b_{2}^{\prime}+z_{0} b_{1}^{\prime} & b_{3}^{\prime}+z_{1} b_{1}^{\prime}+\lambda z_{0} b_{2}^{\prime}\end{array}\right)$. We have $b_{1}^{\prime}=b_{1}$, and choose $y_{1}$ so that $b_{2}^{\prime}=0$, and $y_{2}$ so that $b_{3}^{\prime}=0$. Hence $B$ is reduce to $\left(\begin{array}{ccccc}1 & a_{0} & b_{1} & & \\ & 1 & & & \\ & & 1 & c & \\ & & & 1 & d \\ & & & & 1\end{array}\right)$, and $Z_{U_{5}\left(\mathbf{F}_{q}\right)}(A, B)=\left\{\left(\begin{array}{ccccc}1 & x_{0} & \frac{b_{1}}{c} z_{0} & \frac{b_{1}}{d} z_{1} & y_{3} \\ & 1 & & & \\ & 1 & z_{0} & z_{1} \\ & & 1 & \lambda z_{0} \\ & & & 1\end{array}\right)\right\}$. This centralizer is 4 dimensional, and commutative. Thus $(A, B)$ is of type $R_{3}$, and there are $(q-1)^{2} q^{2}$ such branches.

With this, we have no other cases to analyse. So from the calculations, we have:

- $q^{2}$ branches of type $A_{5}$.

- $q(q-1)+q(q-1)=2 q(q-1)$ branches of type $B_{4}$.

- $q(q-1)+q(q-1)^{2}+q^{3}(q-1)=q^{4}-q^{2}$ branches of type $B_{6}$. 
- $q^{2}(q-1)$ branches of type $R_{2}$.

- $q(q-1)^{2}+q^{2}(q-1)^{2}=q(q-1)\left(q^{2}-1\right)$ branches of type $R_{3}$, and

- $q(q-1)^{2}$ branches of the new type $U N T_{1}$.

7.2. Branching of type $B$. Now we look at the $B$ types and decide its branching.

Proposition 7.7. An upper unitriangular matrix of type $B_{1}$ has the following branches:

\begin{tabular}{c|c||c|c}
\hline Branch & No. of Branches & Branch & No. of Branches \\
\hline$B_{1}$ & $q^{2}$ & $R_{3}$ & $(q-1)^{2}\left(q^{2}+q+1\right)$ \\
$A_{2}$ & $3 q^{2}-3 q$ & $U N T_{1}$ & $(q-1)^{2}$ \\
$R_{1}$ & $2 q^{3}-4 q+2$ & $U N T_{2}$ & $2 q^{2}-2 q$ \\
$R_{2}$ & $q(q-1)^{2}(q+2)$ & $U N T_{3}$ & $(q-1)^{3}$. \\
$B_{6}$ & $q^{2}(q-1)$ & & \\
\hline
\end{tabular}

We have seen $\overline{U N T_{1} \text { earlier. There are two more new types here } U N T_{2}}$ with centralizer

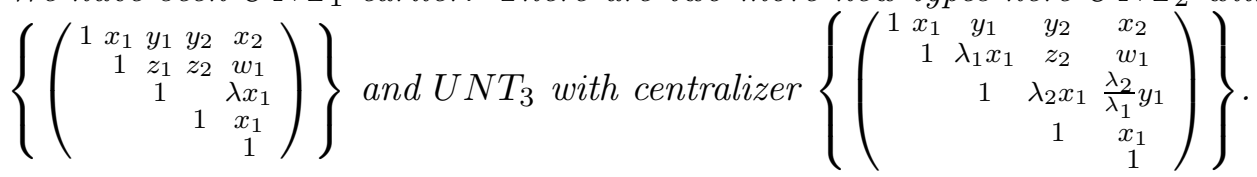

Proof. A matrix of type $B_{1}$ has the canonical form: $\left(\begin{array}{ccccc}1 & & & a & \\ & 1 & & & b \\ & & 1 & & \\ & & & 1 & \\ & & & 1\end{array}\right)$. We may take

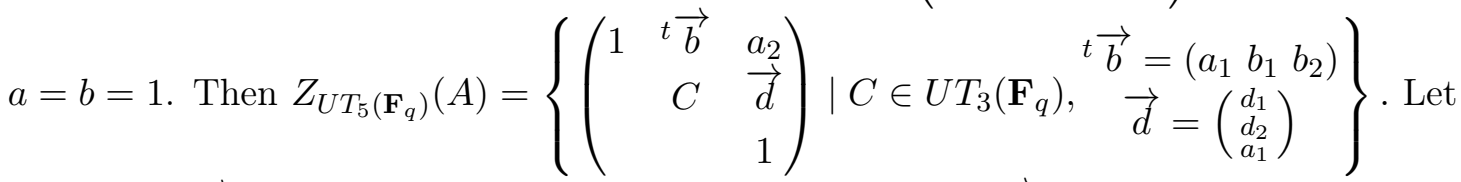
$B=\left(\begin{array}{ccc}1 & t \vec{b} & a_{2} \\ & C & \vec{d} \\ & & 1\end{array}\right), X=\left(\begin{array}{ccc}1 & { }^{t} \vec{y} & x_{2} \\ & Z & \vec{w} \\ & & 1\end{array}\right)$, and $B^{\prime}=\left(\begin{array}{ccc}1 & { }^{t} \vec{b}^{\prime} & a_{2}^{\prime} \\ & C^{\prime} & \overrightarrow{d^{\prime}} \\ & & 1\end{array}\right)=X B X^{-1}$. Then $X B=B^{\prime} X$ leads to firstly $Z C=C^{\prime} Z$, so we might as well take $C$ to be a conjugacy class representative in $U T_{3}\left(\mathbf{F}_{q}\right)$, and $Z$, a centralizer matrix of $C$. We also get in ${ }^{t} \vec{b}$, and $\vec{d}, a_{1}^{\prime}=a_{1}$, and the following equations:

$$
\begin{aligned}
& \left(\begin{array}{lll}
a_{1} & b_{1} & b_{2}
\end{array}\right)+\left(\begin{array}{lll}
x_{1} & y_{1} & y_{2}
\end{array}\right) C=\left(\begin{array}{lll}
a_{1}^{\prime} & b_{1}^{\prime} & b_{2}^{\prime}
\end{array}\right) Z+\left(\begin{array}{lll}
x_{1} & y_{1} & y_{2}
\end{array}\right) \\
& Z\left(\begin{array}{l}
d_{1} \\
d_{2} \\
a_{1}
\end{array}\right)+\left(\begin{array}{l}
w_{1} \\
w_{2} \\
x_{1}
\end{array}\right)=\left(\begin{array}{l}
d_{1}^{\prime} \\
d_{2}^{\prime} \\
a_{1}
\end{array}\right)+C\left(\begin{array}{l}
w_{1} \\
w_{2} \\
x_{1}
\end{array}\right) \\
& a_{2}+x_{1} d_{1}+y_{1} d_{2}+y_{2} d_{1}=a_{27}^{\prime}+a_{1} w_{1}+b_{1}^{\prime} w_{2}+b_{2}^{\prime} x_{1}
\end{aligned}
$$


We look at two main cases: $a_{1}=0$, and $a_{1} \neq 0$.

Case $a_{1}=0$ : First we look at $b_{1}=b_{2}=d_{1}=d_{2}=0$. Here Equation 7.10 reduces to $\left(\begin{array}{lll}x_{1} & y_{1} & y_{2}\end{array}\right) C=\left(\begin{array}{lll}x_{1} & y_{1} & y_{2}\end{array}\right)$, Equation 7.11 reduces to $C\left(\begin{array}{lll}w_{1} & w_{2} & x_{1}\end{array}\right)=$ $\left(\begin{array}{lll}w_{1} & w_{2} & x_{1}\end{array}\right)$, and from Equation 7.12, we have $a_{2}^{\prime}=a_{2}$.

When $C=I_{3}$, Equations 7.10 and 7.11 are void, and we have $B=\left(\begin{array}{ccccc}1 & & & & a_{2} \\ & 1 & & & \\ & 1 & & \\ & & 1 & \\ & & & & 1\end{array}\right)$,

with $Z_{U T_{5}\left(\mathbf{F}_{q}\right)}(A, B)=Z_{U T_{5}\left(\mathbf{F}_{q}\right)}(A)$. Thus $(A, B)$ is of type $B_{1}$, and there are $q$ such branches.

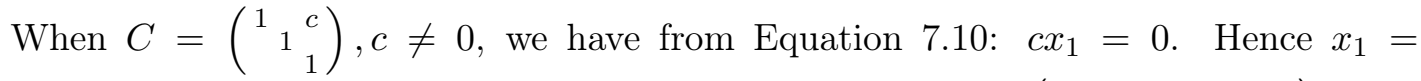
0. With this Equation 7.11 becomes void. So, we have $B=\left(\begin{array}{cccccc}1 & & & & a_{2} \\ & 1 & & c & \\ & 1 & & \\ & & 1 & \\ & & & & 1\end{array}\right)$, and $Z_{U T_{5}\left(\mathbf{F}_{q}\right)}(A, B)=\left\{\left(\begin{array}{ccccc}1 & & y_{1} & y_{2} & x_{2} \\ & 1 & z_{1} & z_{2} & w_{1} \\ & & 1 & z_{3} & w_{2} \\ & & & 1 & \\ & & & & 1\end{array}\right)\right\}$, which is the centralizer of a canonical form of type $A_{2} .(A, B)$ is a branch of type $A_{2}$, and there are $q(q-1)$ such branches.

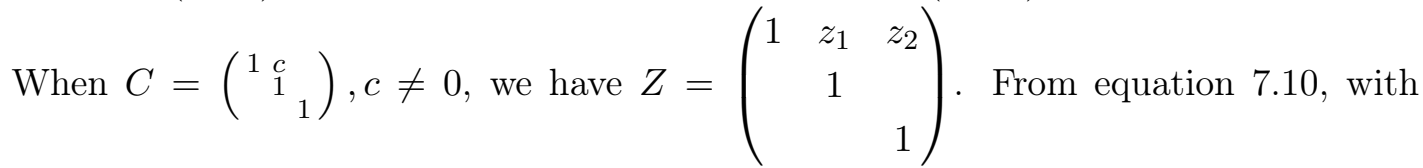
this $C$, we get: $\left(\begin{array}{lll}x_{1} & y_{1}+c x_{1} & y_{2}\end{array}\right)=\left(\begin{array}{lll}x_{1} & y_{1} & y_{2}\end{array}\right)$, which leaves us with $x_{1}=0$. Equation 7.11 becomes: $\left(\begin{array}{l}w_{1} \\ w_{2}\end{array}\right)=\left(\begin{array}{c}w_{1}+c w_{2} \\ w_{2}\end{array}\right)$, thus we have $w_{2}=0$. So we have $B=I_{5}+c E_{24}+a_{2} E_{15}$, with $Z_{U T_{5}\left(\mathbf{F}_{q}\right)}(A, B)=\left\{\left(\begin{array}{ccccc}1 & & y_{1} & y_{2} & x_{2} \\ & 1 & z_{1} & z_{2} & w_{1} \\ & & 1 & & \\ & & & 1 & \\ & & & 1\end{array}\right)\right\}$, which is the centralizer of a matrix of type $R_{1}$. Thus $(A, B)$ is of type $R_{1}$, and there are $q(q-1)$ such branches. 


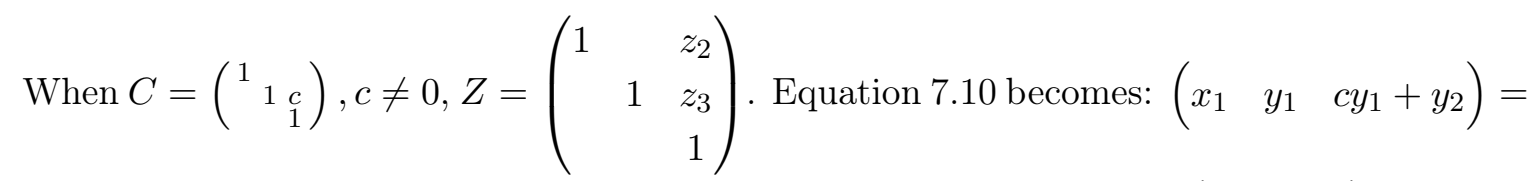
$\left(\begin{array}{lll}x_{1} & y_{1} & y_{2}\end{array}\right)$, which leaves us with $y_{1}=0$. Equation 7.11 becomes $\left(\begin{array}{c}w_{1} \\ w_{2}+c x_{1} \\ x_{1}\end{array}\right)=$ $\left(\begin{array}{l}w_{1} \\ w_{2} \\ x_{1}\end{array}\right)$, which leads to $x_{1}=0$. So $B=I_{5}+a_{2} E_{15}+c E_{34}$, with $Z_{U T_{5}\left(\mathbf{F}_{q}\right)}(A, B)=$

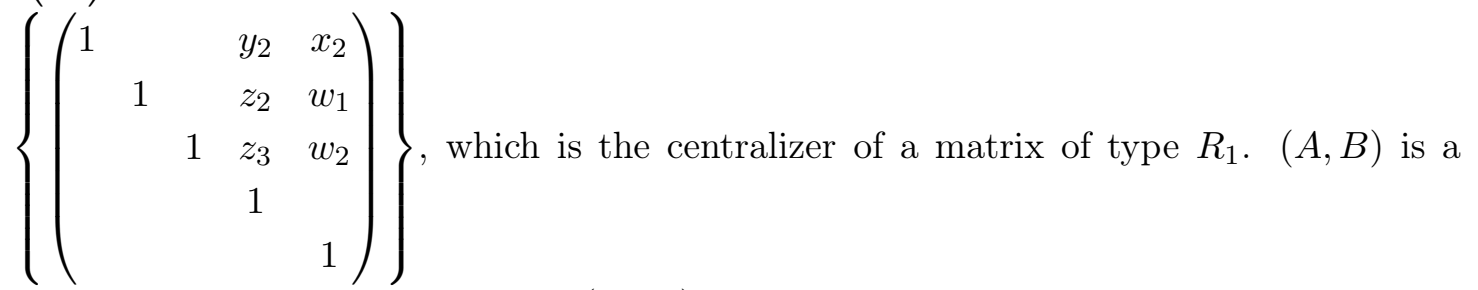
branch of type $R_{1}$, and there are $q(q-1)$ such branches.

When $C=\left(\begin{array}{ccc}1 & c & \\ & 1 & d \\ & & 1\end{array}\right), c_{1}, c_{2} \neq 0, Z=\left(\begin{array}{ccc}1 & z_{1} & z_{2} \\ & 1 & \frac{d}{c} z_{1} \\ & & 1\end{array}\right)$. Equation 7.10 becomes $\left(\begin{array}{lll}x_{1} & c x_{1}+y_{1} & d y_{1}+y_{2}\end{array}\right)=\left(\begin{array}{lll}x_{1} & y_{2} & y_{2}\end{array}\right)$, which leaves us with $x_{1}=y_{1}=0$. Equation 7.11 becomes $\left(\begin{array}{c}w_{1}+c w_{2} \\ w_{2}\end{array}\right)=\left(\begin{array}{c}w_{1} \\ w_{2}\end{array}\right)$, which leaves us with $w_{2}=0$. Hence $B=$ $I_{5}+a_{2} E_{15}+c E_{23}+d E_{34}$, and $Z_{U T_{5}\left(\mathbf{F}_{q}\right)}(A, B)=\left\{\left(\begin{array}{ccccc}1 & & & y_{2} & x_{2} \\ & 1 & z_{1} & z_{2} & w_{1} \\ & & 1 & \frac{d}{c} z_{1} & \\ & & 1 & \\ & & & & 1\end{array}\right)\right\}$, which is the centralizer of a matrix of type $R_{2} .(A, B)$ is a branch of type $R_{2}$, and there are $q(q-1)^{2}$ branches.

When $\left(\left(b_{1}, b_{2}\right),\left(d_{1}, d_{2}\right)\right) \neq(\overrightarrow{0}, \overrightarrow{0})$ :

We start with $C=I_{3}$ : Thus Equation 7.10 becomes $\left(\begin{array}{lll}0 & b_{1} & b_{2}\end{array}\right)=\left(\begin{array}{lll}0 & b_{1}^{\prime} & b_{1}^{\prime} z_{3}+b_{2}^{\prime}\end{array}\right)$. We have $b_{1}^{\prime}=b_{1}$, and thus $b_{2}=b_{2}^{\prime}+b_{1} z_{3}$. First, when $b_{1} \neq 0$, we choose $z_{3}$ so that $b_{2}^{\prime}=0$. Thus, on replacing $b_{2}$ by $b_{2}^{\prime}=0$ in the equation above, we have $z_{3}=0$. So Equation 7.11 boils down to $\left(\begin{array}{c}d_{1}+z_{1} d_{2} \\ d_{2} \\ 0\end{array}\right)=\left(\begin{array}{c}d_{1}^{\prime} \\ d_{2}^{\prime} \\ 0\end{array}\right)$. So we have $d_{2}^{\prime}=d_{2}$. So, again, over here, when $d_{2} \neq 0$, choose $z_{1}$ such that $d_{1}^{\prime}=0$. With these, Equation 7.12 becomes $y_{1} d_{2}+a_{2}=a_{2}^{\prime}+w_{2} b_{1}$. So, choose $w_{2}$ such that $a_{2}^{\prime}=0$. So, $B$ is reduced to 
$I_{5}+b_{1} E_{13}+d_{2} E_{35}$, and $Z_{U T_{5}\left(\mathbf{F}_{q}\right)}(A, B)=\left\{\left(\begin{array}{ccccc}1 & x_{1} & y_{1} & y_{2} & x_{2} \\ & 1 & & z_{2} & w_{1} \\ & & & \frac{d_{2}}{b_{1}} y_{1} \\ & & & 1 & x_{1} \\ & & & & 1\end{array}\right)\right\}$. This centralizer is isomorphic to that of the new type $U N T_{1}$ (as seen in Proposition 7.4), via the isomorphism that maps generators to generators, and extended product-wise.

When $b_{1} \neq 0$, and $d_{2}=0$, we have $d_{1}^{\prime}=d_{1}$, and Equation 7.12 becomes $x_{1} d_{1}+=$ $a_{2}^{\prime}+b_{1} w_{2}$. So, we choose $w_{2}$ such that $a_{2}^{\prime}=0$. So $B$ is reduced to $\left(\begin{array}{lllll}1 & & b_{1} & & \\ & 1 & & & d_{1} \\ & 1 & & \\ & & 1 & \\ & & & 1\end{array}\right)$, and $Z_{U T_{5}\left(\mathbf{F}_{q}\right)}(A, B)=\left\{\left(\begin{array}{ccccc}1 & x_{1} & y_{1} & y_{2} & x_{2} \\ & 1 & z_{1} & z_{2} & w_{1} \\ & & 1 & & \frac{d_{1}}{b_{1}} x_{1} \\ & & & 1 & x_{1} \\ & & & & 1\end{array}\right)\right\}$. Thus $(A, B)$ is of a new type, which we call $U N T_{2}$, and there are $q(q-1)$ such branches.

When $b_{1}=0, b_{2}^{\prime}=b_{2}$. First, when $d_{2} \neq 0$, we choose $z_{1}$ so that $d_{1}^{\prime}=0$, and hence Equation 7.12 becomes $a_{2}+y_{1} d_{2}=a_{2}^{\prime} z+b_{2} x_{1}$. As $d_{2} \neq 0$, choose $y_{1}$ so that $a_{2}^{\prime}=0$. Hence $B$ is reduced to $\left(\begin{array}{ccccc}1 & & & b_{2} & \\ & 1 & & & \\ & & 1 & & d_{2} \\ & & & 1 & \\ & & & & 1\end{array}\right)$, and $Z_{U T_{5}\left(\mathbf{F}_{q}\right)}(A, B)=\left\{\left(\begin{array}{ccccc}1 & x_{1} & \frac{b_{2}}{d_{2}} x_{1} & y_{2} & x_{2} \\ & 1 & & z_{2} & w_{1} \\ & & 1 & z_{3} & w_{2} \\ & & 1 & x_{1} \\ & & & & 1\end{array}\right)\right\}$.

This too is of type $U N T_{2}$, and there are $q(q-1)$ such branches.

When $d_{2}=0$, we have $d_{1}^{\prime}=d_{1}$. So Equation 7.12 looks like: $a_{2}+x_{1} d_{1}=a_{2}^{\prime}+x_{1} b_{2}$.

When $b_{2}=d_{1} \neq 0$, we have $a_{2}^{\prime}=a_{2}$, and $B$ is reduced to $\left(\begin{array}{ccccc}1 & & & b_{2} & a_{2} \\ & 1 & & & b_{2} \\ & 1 & & \\ & & 1 & \\ & & & 1\end{array}\right)$, and $Z_{U T_{5}\left(\mathbf{F}_{q}\right)}(A, B)=Z_{U T_{5}\left(\mathbf{F}_{q}\right)}(A)$. Thus $(A, B)$ is of type $B_{1}$, and there are $q(q-1)$ such branches. 
When $b_{2} \neq d_{1}$, choose an $x_{1}$ such that $a_{2}^{\prime}=0$. So, $B$ is boiled down to $\left(\begin{array}{ccccc}1 & & & b_{1} & \\ & 1 & & & d_{2} \\ & 1 & & \\ & & 1 & \\ & & & & 1\end{array}\right)$, and $Z_{U T_{5}\left(\mathbf{F}_{q}\right)}(A, B)=\left\{\left(\begin{array}{ccccc}1 & & y_{1} & y_{2} & x_{2} \\ & 1 & z_{1} & z_{2} & w_{1} \\ & & 1 & z_{3} & w_{2} \\ & & & 1 & \\ & & & & 1\end{array}\right)\right\}$, which is the centralizer of a matrix of type $A_{2}$, and there are $q(q-1)$ such branches.

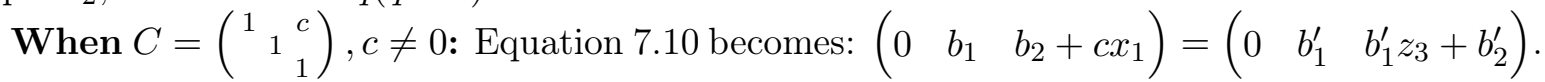
We have $b_{1}^{\prime}=b_{1}$, and as $c \neq 0$, we can choose $x_{1}$ so that $b_{2}^{\prime}=0$. So, on replacing $b_{2}$ by 0 in the above equation, we have $x_{1}=\frac{b_{1}}{c} z_{3}$.

Then from Equation 17.11, we have $\left(\begin{array}{c}d_{1}+d_{2} z_{1} \\ d_{2} \\ 0\end{array}\right)=\left(\begin{array}{c}d_{1}^{\prime}+b_{1} z_{3} \\ d_{2}^{\prime} \\ 0\end{array}\right)$. First, when $b_{1} \neq 0$, we choose a $z_{3}$ so that $d_{1}^{\prime}=0$. With these, Equation 7.12 becomes: $a_{2}+y_{1} d_{2}=a_{2}^{\prime}+b_{1} w_{2}$. As $b_{1} \neq 0$, choose $w_{2}$ so that $a_{2}^{\prime}=0$. So $B$ is reduced to

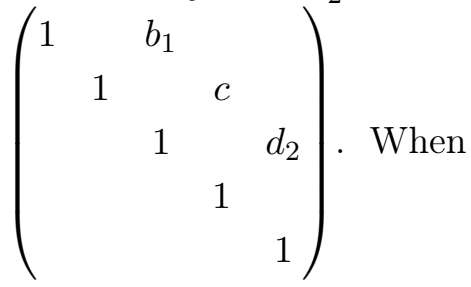
$d_{2} \neq 0 Z_{U T_{5}\left(\mathbf{F}_{q}\right)}(A, B)=\left\{\left(\begin{array}{ccccc}1 & \frac{d_{2}}{c} z_{1} & y_{1} & y_{2} & x_{2} \\ & 1 & z_{1} & z_{2} & w_{1} \\ & & 1 & \frac{d_{2}}{b_{1}} z_{1} & \frac{d_{2}}{b_{1}} y_{1} \\ & & & 1 & \frac{d_{2}}{c} z_{1} \\ & & & & 1\end{array}\right)\right\}$. This isn't isomorphic to the centralizer of any matrix in $U T_{5}\left(\mathbf{F}_{q}\right)$, hence $(A, B)$ is of a new type $U N T_{3}$, and there are $(q-1)^{3}$ such branches. When $d_{2}=0, Z_{U T_{5}\left(\mathbf{F}_{q}\right)}(A, B)=\left\{\left(\begin{array}{ccccc}1 & & y_{1} & y_{2} & x_{2} \\ & 1 & z_{1} & z_{2} & w_{1} \\ & & 1 & & \\ & & & 1 & \\ & & & & 1\end{array}\right)\right\}$. Hence $(A, B)$ is of type $R_{1}$, and there are $(q-1)^{2}$ such branches.

When $b_{1}=0$, we have $x_{1}=\frac{b_{1}}{c} z_{3}=0$, we have $d_{1}^{\prime}=d_{1}+z_{1} d_{2}$. When $d_{2} \neq 0$, choose $z_{1}$ such that $d_{1}^{\prime}=0$. Equation 7.12 becomes $a_{2}+y_{1} d_{2}=a_{2}^{\prime}$. Choose $y_{1}$ so that $a_{2}^{\prime}=0$. 
So, $B$ is reduced to $\left(\begin{array}{ccccc}1 & & & & \\ & 1 & & c & \\ & & 1 & & d_{2} \\ & & & 1 & \\ & & & & 1\end{array}\right)$, and $Z_{U T_{5}\left(\mathbf{F}_{q}\right)}(A, B)=\left\{\left(\begin{array}{ccccc}1 & & & y_{2} & x_{2} \\ & 1 & & z_{2} & w_{1} \\ & 1 & z_{3} & w_{2} \\ & & 1 & \\ & & & & 1\end{array}\right)\right\}$.

$(A, B)$ is of type $R_{1}$, and there are $(q-1)^{2}$ such branches.

When $b_{1}=0$, and $d_{2}=0$. Then $d_{1}^{\prime}=d_{1}$, which is $\neq 0$ Then from Equation 7.12 ,

we simply have $a_{2}^{\prime}=a_{2}$, and $B$ is reduced to $\left(\begin{array}{ccccc}1 & & & & a_{2} \\ & 1 & & c & d_{1} \\ & 1 & & \\ & & 1 & \\ & & & 1\end{array}\right)$. $Z_{U T_{5}\left(\mathbf{F}_{q}\right)}(A, B)=$

$\left\{\left(\begin{array}{ccccc}1 & & y_{1} & y_{2} & x_{2} \\ & 1 & z_{1} & z_{2} & w_{1} \\ & & 1 & z_{3} & w_{2} \\ & & & 1 & \\ & & & & 1\end{array}\right)\right\} .(A, B)$ is of type $A_{2}$, and there are $q(q-1)$ such branches.

When $C\left(\begin{array}{ccc}1 & c & \\ & 1 & \\ & & 1\end{array}\right), c \neq 0: Z=\left(\begin{array}{ccc}1 & z_{1} & z_{2} \\ & 1 & \\ & & 1\end{array}\right)$. Equation 7.10 becomes $\left(\begin{array}{lll}0 & b_{1}+c x_{1} & b-2\end{array}\right)=$

$\left(\begin{array}{lll}0 & b_{1}^{\prime} & b_{2}^{\prime}\end{array}\right)$. We get that $b_{2}^{\prime}=b_{2}$. We choose $x_{1}$ so that $b_{1}^{\prime}=0$. Thus, on replacing $b_{1}$ by 0 , and equating the above equation, we have $x_{1}=0$. Equation 7.11 becomes $\left(\begin{array}{c}d_{1}+z_{1} d_{2} \\ d_{2} \\ 0\end{array}\right)=\left(\begin{array}{c}c w_{2}+d_{1}^{\prime} \\ d_{2}^{\prime} \\ 0\end{array}\right)$. Again, over here, we choose $w_{2}$ such that $d_{1}^{\prime}=0$. Now, on substituting $b_{1}$ with 0 , we have $w_{2}=\frac{d_{1}}{c} z_{1}$. So, Equation 7.12 becomes $a_{2}+y_{1} d_{2}=a_{2}^{\prime}$.

When $d_{2} \neq 0$, choose $y_{1}$ such that $a_{2}^{\prime}=$. So $B$ is reduced to $\left(\begin{array}{llllll}1 & & & b_{2} & \\ & 1 & c & & \\ & & 1 & & d_{2} \\ & & & 1 & \\ & & & & 1\end{array}\right)$, and $Z_{U T_{5}\left(\mathbf{F}_{q}\right)}(A, B)=\left\{\left(\begin{array}{ccccc}1 & & & y_{2} & x_{2} \\ & 1 & z_{1} & z_{2} & w_{1} \\ & & 1 & & \frac{d_{2}}{c} z_{1} \\ & & & 1 & \\ & & & & 1\end{array}\right)\right\} .(A, B)$ is therefore of type $R_{2}$, and there are $q(q-1)^{2}$ such branches. 
When $d_{2}=0$, and $b_{2} \neq 0$. Then Equation 7.12 becomes $a_{2}^{\prime}=a_{2}$. So, $B$ is reduced to

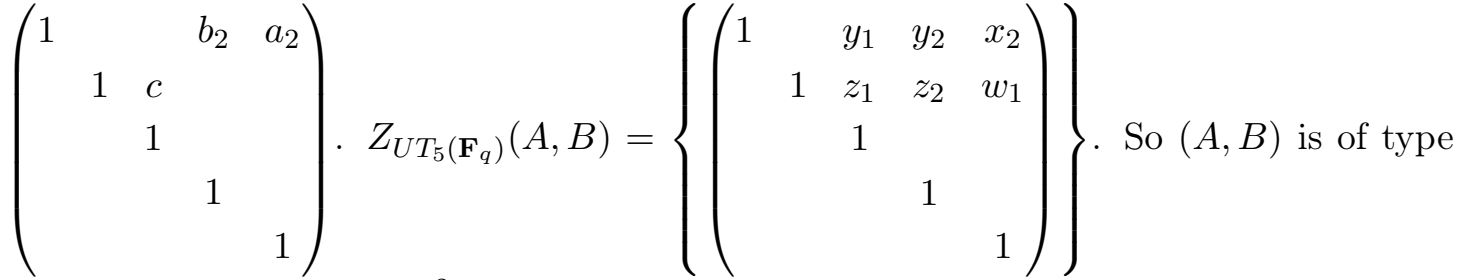
$R_{1}$, and there are $q(q-1)^{2}$ branches of this type.

When $C\left(\begin{array}{ccc}1 & 1 & \\ & 1 & c\end{array}\right), c \neq 0: Z=\left(\begin{array}{ccc}1 & & z_{2} \\ & 1 & z_{3} \\ & & 1\end{array}\right)$. Equation 7.10 becomes $\left(\begin{array}{llll}0 & b_{1} & b_{2}+c y_{1}\end{array}\right)=$ $\left(\begin{array}{lll}0 & b_{1}^{\prime} & b_{1}^{\prime} z_{3}+b_{2}^{\prime}\end{array}\right) . b_{1}^{\prime}=b_{1}$. Choose $y_{1}$ such that $b_{2}^{\prime}=0$. So, on substituting $b_{2}$ with 0 , we have $y_{1}=\frac{b_{1}}{c} z_{3}$. Equation 7.11 becomes $\left(\begin{array}{c}d_{1}+d_{2} z_{1} \\ d_{2} \\ 0\end{array}\right)=\left(\begin{array}{c}d_{1}^{\prime} \\ d_{2}^{\prime}+c x_{1} \\ 0\end{array}\right)$. Choose $x_{1}$ such that $d_{2}^{\prime}=0$. So $d_{1}^{\prime}=d_{1}$. Hence, on replacing $d_{2}$ by 0 , we get $x_{1}=0$. Hence, Equation 7.12 becomes $a_{2}=a_{2}^{\prime}+b_{1} w_{2}$. When $b_{1} \neq 0$, choose $w_{2}$ such that $a_{2}^{\prime}=0$. Thus, $B$ is reduced to $\left(\begin{array}{ccccc}1 & & b_{1} & & \\ & 1 & & & \\ & & 1 & c & d_{2} \\ & & & 1 & \\ & & & & 1\end{array}\right)$, and $Z_{U T_{5}\left(\mathbf{F}_{q}\right)}(A, B)=\left\{\left(\begin{array}{ccccc}1 & & \frac{b_{1}}{c} z_{3} & y_{2} & x_{2} \\ & 1 & & z_{2} & w_{1} \\ & & 1 & z_{3} & \\ & & 1 & \\ & & & 1\end{array}\right)\right\}$.

So $(A, B)$ is of type $R_{2}$, and there are $q(q-1)^{2}$ such branches.

When $b_{1}=0$, and $d_{1}^{\prime}=d_{1}$, we get from Equation 7.12, we get $a_{2}^{\prime}=a_{2}$. Hence $B$ is reduced to $\left(\begin{array}{ccccc}1 & & & & a_{2} \\ & 1 & & & \\ & & 1 & c & d_{2} \\ & & & 1 & \\ & & & & 1\end{array}\right)$, and $Z_{U T_{5}\left(\mathbf{F}_{q}\right)}(A, B)=\left\{\left(\begin{array}{ccccc}1 & & & y_{2} & x_{2} \\ & 1 & z_{2} & w_{1} \\ & & 1 & z_{3} & w_{2} \\ & & 1 & \\ & & & & 1\end{array}\right)\right\}$.

is of type $R_{1}$, and there are $q(q-1)^{2}$ such branches.

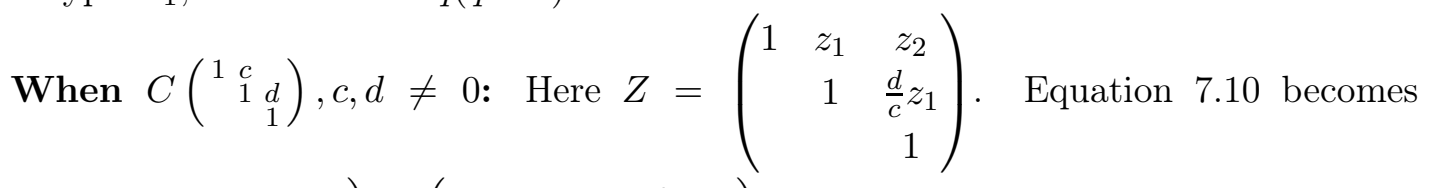

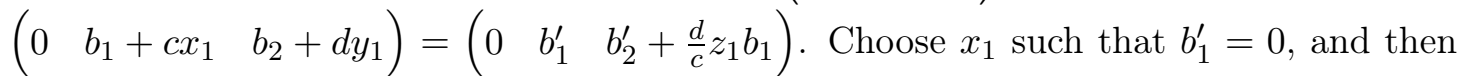
choose $y_{1}$ such that $b_{2}^{\prime}=0$. So, on substituting $b_{1}$ with 0 , we get $x_{1}=0$. Then, on substituting $b_{2}$ with 0 , we get $y_{1}=0$. Equation 7.11 becomes $\left(\begin{array}{c}d_{1}+d_{2} z_{1} \\ d_{2} \\ 0\end{array}\right)=$ 
$\left(\begin{array}{c}d_{1}^{\prime}+c w_{2} \\ d_{2}^{\prime} \\ 0\end{array}\right)$. We have $d_{2}^{\prime}=d_{2} \neq 0$, choose $w_{2}$ such that $d_{1}^{\prime}=0$. Thus, with these Equation 7.12 becomes $a_{2}^{\prime}=a_{2}$. So $B$ becomes $\left(\begin{array}{rrrrr}1 & & & & a_{2} \\ & 1 & c & & \\ & & 1 & d & d_{2} \\ & & & 1 & \\ & & & & 1\end{array}\right)$. $Z_{U T_{5}\left(\mathbf{F}_{q}\right)}(A, B)=$ $\left\{\left(\begin{array}{ccccc}1 & & & y_{2} & x_{2} \\ & 1 & \frac{d}{c} z_{1} & z_{2} & w_{1} \\ & 1 & z_{1} & \frac{d_{2}}{c} z_{1} \\ & & 1 & \\ & & & & 1\end{array}\right)\right\} .(A, B)$ is of type $R_{2}$. There are $q(q-1)^{3}$ such branches.

When $a_{1} \neq 0$ : We now look at the branches, where the entry $a_{1} \neq 0$.

When $C=I_{3}$ : Equation 7.10 becomes $\left(\begin{array}{lll}a_{1} & b_{1} & b_{2}\end{array}\right)=\left(\begin{array}{lll}a_{1} & a_{1} z_{1}+b_{1}^{\prime} & a_{1} z_{2}+b_{1}^{\prime} z_{3}+b_{2}^{\prime}\end{array}\right)$. As $a_{1} \neq 0$, choose $z_{1}$ such that $b_{1}^{\prime}=0$. Then choose $z_{2}$ such that $b_{2}^{\prime}=0$. Now, when we replace $b_{1}$ and $b_{2}$ by 0 in the above equation, we get $z_{1}=z_{2}=0$. Then Equation 7.11 becomes $\left(\begin{array}{c}d_{1} \\ d_{2}+a_{1} z_{3} \\ a_{1}\end{array}\right)=\left(\begin{array}{c}d_{1}^{\prime} \\ d_{2}^{\prime} \\ a_{1}\end{array}\right)$. Choose $z_{3}$ such that $d_{2}^{\prime}=0$. Equation 7.12 becomes $a_{2}+x_{1} d_{1}+a_{1} y_{2}=a_{2}^{\prime}+a_{1} w_{1}$. Choose $w_{1}$ such that $a_{2}^{\prime}=0$. So, $B$ is boiled down to $\left(\begin{array}{ccccc}1 & a_{1} & & & \\ & 1 & & & d_{1} \\ & & 1 & & \\ & & 1 & a_{1} \\ & & & & 1\end{array}\right)$, and $Z_{U T_{5}\left(\mathbf{F}_{q}\right)}(A, B)=\left\{\left(\begin{array}{ccccc}1 & x_{1} & y_{1} & y_{2} & x_{2} \\ & 1 & & & y_{2}+\frac{d_{1}}{a_{1}} x_{1} \\ & & & \\ & & & \\ & & & 1 & x_{1} \\ & & & & 1\end{array}\right)\right\}$. Now, we see that this centralizer is of size $q^{5}$, hence we expect it to be a commutative one. But it isn't. We also know that no matrix in $U T_{5}\left(\mathbf{F}_{q}\right)$ has a non-commutative centralizer of size $q^{5}$, and it is isomorphic to that of the type $B_{6}$. Thus, $(A, B)$ is of type $B_{6}$, and there are $q(q-1)$ such branches.

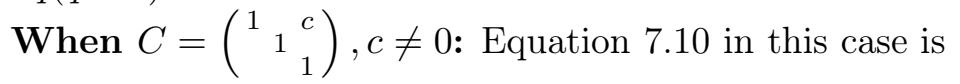

$$
\left(\begin{array}{lll}
a_{1} & b_{1} & b_{2}+c x_{1}
\end{array}\right)=\left(\begin{array}{lll}
a_{1} & a_{1} z_{1}+b_{1}^{\prime} & a_{1} z_{2}+b_{1}^{\prime} z_{3}+b_{2}^{\prime}
\end{array}\right)
$$

Choose $z_{1}$ so that $b_{1}^{\prime}=0$. Then, we choose $z_{2}$ so that $b_{2}^{\prime}=0$. Thus, on substituting $b_{1}$ and $b_{2}$ with 0 , we get $z_{1}=0$, and $z_{2}=\frac{c}{a_{1}} x_{1}$. Then Equation 7.11 becomes $\left(\begin{array}{c}d_{1} \\ d_{2}+z_{3} a_{1} \\ a_{1}\end{array}\right)=$ 
$\left(\begin{array}{l}d_{1}^{\prime} \\ d_{2}^{\prime} \\ a_{1}\end{array}\right)$. Choose $z_{3}$ such that $d_{2}^{\prime}=0$. Equation 7.12 becomes $a_{2}+x_{1} d_{1}+a_{1} y_{2}=a_{2}^{\prime}+$ $a_{1} w_{1}$. Choose $w_{1}$ such that $a_{2}^{\prime}=0$. So $B$ boils down to $\left(\begin{array}{ccccc}1 & a_{1} & & & \\ & 1 & & c & d_{1} \\ & & 1 & & \\ & & 1 & a_{1} \\ & & & 1\end{array}\right)$, and $Z_{U T_{5}\left(\mathbf{F}_{q}\right)}(A, B)=\left\{\left(\begin{array}{ccccc}1 & x_{1} & y_{1} & y_{2} & x_{2} \\ & 1 & & \frac{c}{a_{1}} x_{1} & \frac{d_{1}}{a_{1}} x_{1}+y_{2} \\ & & 1 & & w_{2} \\ & & & 1 & x_{1} \\ & & & & 1\end{array}\right)\right\}$. This too is a branch of type $B_{6}$, and there are $q(q-1)^{2}$ such branches.

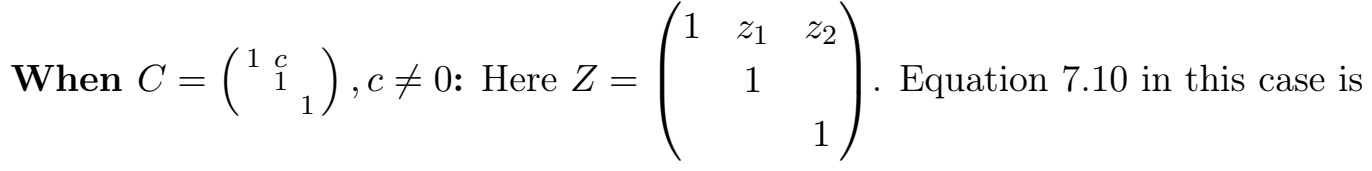

$$
\begin{aligned}
& \left(\begin{array}{lll}
a_{1} & b_{1}+c x_{1} & b_{2}
\end{array}\right)=\left(\begin{array}{lll}
a_{1} & a_{1} z_{1}+b_{1}^{\prime} & a_{1} z_{2}+b_{2}^{\prime}
\end{array}\right) .
\end{aligned}
$$

Choose $z_{1}$ so that $b_{1}^{\prime}=0$, and choose $z_{2}$ so that $b_{2}^{\prime}=0$. Thus, on substituting $b_{1}$ and $b_{2}$ with 0 , we get $z_{1}=\frac{c}{a_{1}} x_{1}$, and $z_{2}=0$. Then Equation 7.11 becomes $\left(\begin{array}{c}d_{1}+\frac{c}{a_{1}} x_{1} d_{2} \\ d_{2} \\ a_{1}\end{array}\right)=$ $\left(\begin{array}{c}d_{1}^{\prime}+c w_{2} \\ d_{2}^{\prime} \\ a_{1}\end{array}\right)$. So $d_{2}^{\prime}=d_{2}$, and we choose $w_{2}$ such that $d_{1}^{\prime}=0$. Equation 7.12 becomes $a_{2}+y_{1} d_{2}+a_{1} y_{2}=a_{2}^{\prime}+a_{1} w_{1}$. Choose $w_{1}$ such that $a_{2}^{\prime}=0$. So $B$ boils down to

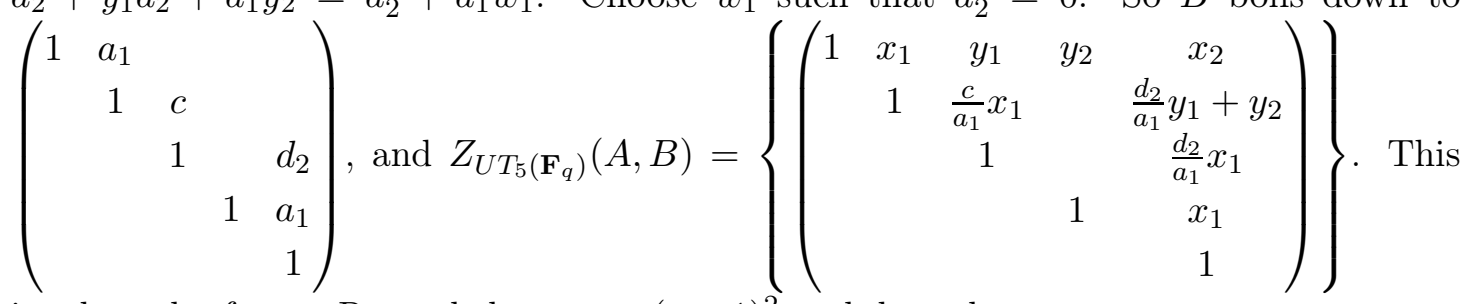
is a branch of type $R_{3}$, and there are $q(q-1)^{2}$ such branches.

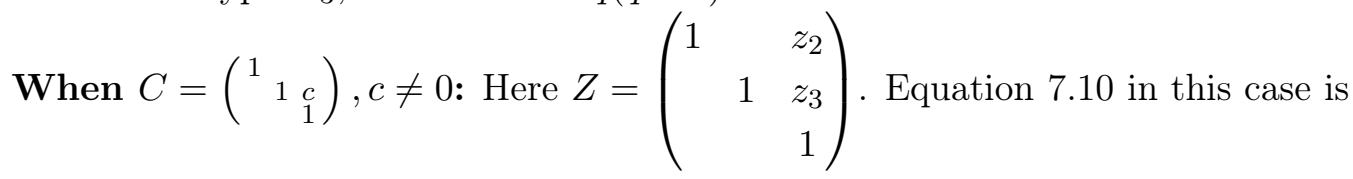

$$
\begin{aligned}
& \left(\begin{array}{lll}
a_{1} & b_{1} & b_{2}+c y_{1}
\end{array}\right)=\left(\begin{array}{lll}
a_{1} & b_{1}^{\prime} & a_{1} z_{2}+b_{1}^{\prime} z_{3}+b_{2}^{\prime}
\end{array}\right) .
\end{aligned}
$$


We get $b_{1}^{\prime}=b_{1}$. Choose $y_{1}$ so that $b_{2}^{\prime}=0$. Thus, on substituting $b_{2}$ with 0 , we get $y_{1}=\frac{a_{1}}{c} z_{2}+\frac{b_{1}}{c} z_{3}$. Then Equation 7.11 becomes $\left(\begin{array}{c}d_{1}+a_{1} z_{2} \\ d_{2}+a_{1} z_{3} \\ a_{1}\end{array}\right)=\left(\begin{array}{c}d_{1}^{\prime} \\ d_{2}^{\prime}+c x_{1} \\ a_{1}\end{array}\right)$. Choose $z_{2}$ such that $d_{1}^{\prime}=0$, and $z_{3}$ such that $d_{2}^{\prime}=0$. Equation 7.12 becomes $a_{2}+a_{1} y_{2}=$ $a_{2}^{\prime}+b_{1} w_{2}+a_{1} w_{1}$. Choose $w_{1}$ such that $a_{2}^{\prime}=0$. So $B$ boils down to $\left(\begin{array}{ccccc}1 & a_{1} & b_{1} & & \\ & 1 & & & \\ & 1 & c & \\ & & 1 & a_{1} \\ & & & 1\end{array}\right)$, and $Z_{U T_{5}\left(\mathbf{F}_{q}\right)}(A, B)=\left\{\left(\begin{array}{ccccc}1 & x_{1} & \frac{b_{1}}{a_{1}} x_{1} & y_{2} & x_{2} \\ & 1 & & & y_{2}-\frac{b_{1}}{a_{1}} w_{2} \\ & & 1 & \frac{c}{a_{1}} x_{1} & w_{2} \\ & & & 1 & x_{1} \\ & & & & 1\end{array}\right)\right\}$. This is a branch of type $R_{3}$, and there are $q(q-1)^{2}$ such branches.

And, lastly:

When $C=\left(\begin{array}{ccc}1 & c & \\ & 1 & d \\ & & 1\end{array}\right), c, d \neq 0$ : Here $Z=\left(\begin{array}{ccc}1 & z_{1} & z_{2} \\ & 1 & \frac{d}{c} z_{1} \\ & & 1\end{array}\right)$. Equation 7.10 in this case is

$$
\left(\begin{array}{lll}
a_{1} & b_{1}+c x_{1} & b_{2}+d y_{1}
\end{array}\right)=\left(\begin{array}{lll}
a_{1} & a_{1} z_{1}+b_{1}^{\prime} & a_{1} z_{2}+\frac{d}{c} b_{1}^{\prime} z_{1}+b_{2}^{\prime}
\end{array}\right) .
$$

Choose $x_{1}$ so that $b_{1}^{\prime}=0$, and choose $y_{1}$ so that $b_{2}^{\prime}=0$. Thus, on substituting $b_{1}$ and $b_{2}$ with 0 , we get $x_{1}=\frac{a_{1}}{c} z_{1}$, and $y_{1}=\frac{a_{1}}{d} z_{2}$. Then Equation 7.11 becomes $\left(\begin{array}{c}d_{1}+z_{1} d_{2}+z_{2} a_{1} \\ d_{2} \\ a_{1}\end{array}\right)=\left(\begin{array}{c}d_{1}^{\prime}+c w_{2} \\ d_{2}^{\prime} \\ a_{1}\end{array}\right)$. So $d_{2}^{\prime}=d_{2}$, and we choose $w_{2}$ such that $d_{1}^{\prime}=0$. Equation 7.12 becomes $a_{2}+\frac{a_{1}}{d} z_{2} d_{2}+a_{1} y_{2}=a_{2}^{\prime}+a_{1} w_{1}$. Choose $w_{1}$ such that $a_{2}^{\prime}=0$. So $B$ boils down to $\left(\begin{array}{ccccc}1 & a_{1} & & & \\ & 1 & c & & \\ & & 1 & d & d_{2} \\ & & & 1 & a_{1} \\ & & & & 1\end{array}\right)$, and $Z_{U T_{5}\left(\mathbf{F}_{q}\right)}(A, B)=\left\{\left(\begin{array}{ccccc}1 & \frac{a_{1}}{c} z_{1} & \frac{a_{1}}{d} z_{2} & y_{2} & x_{2} \\ & 1 & z_{1} & z_{2} & \frac{d_{2}}{d} z_{2}+y_{2} \\ & & 1 & & \frac{d_{2}}{c} z_{1}+\frac{a_{1}}{c} z_{2} \\ & & 1 & \frac{a_{1}}{c} x_{1} \\ & & & & 1\end{array}\right)\right.$. This is a branch of type $R_{3}$, and there are $q(q-1)^{3}$ such branches.

Proposition 7.8. An upper unitriangular matrix of type $B_{2}$ has 


\begin{tabular}{c|c||c|c}
\hline Branch & No. of Branches & Branch & No. of Branches \\
\hline$B_{2}$ & $q^{2}$ & $R_{3}$ & $(q-1)^{2} q^{2}$ \\
$A_{4}$ & $q^{3}-q$ & $U N T_{1}$ & $q(q-1)^{2}$ \\
$B_{4}$ & $q^{2}-q$ & $U N T_{2}$ & $q^{3}-q^{2}$ \\
$R_{1}$ & $\left(q^{2}-q\right)\left(q^{2}+q-1\right)$ & $B_{6}$ & $q^{4}-q^{3}$. \\
$R_{2}$ & $(q-1)\left(q^{3}-q\right)$ & & \\
\hline
\end{tabular}

Proof. We may take $A=\left(\begin{array}{ccccc}1 & & 1 & & \\ & 1 & & & 1 \\ & & 1 & & \\ & & 1 & \\ & & & & 1\end{array}\right)$. The first of the two canonical forms mentioned for a matrix of type $B_{2}$. For this $A$, we have $Z_{U T_{5}\left(\mathbf{F}_{q}\right)}(A)=\left\{\left(\begin{array}{cccccc}1 & c_{1} & c_{2} & b_{1} & d_{1} \\ & 1 & c_{3} & b_{2} & d_{2} \\ & & 1 & & c_{1} \\ & & & 1 & a \\ & & & & 1\end{array}\right)\right\}$. We rewrite such a matrix as $\left(\begin{array}{ccc}C & \vec{b} & \vec{d} \\ & 0 & c_{1} \\ & 1 & a \\ & & 1\end{array}\right)$, where $C \in U T_{3}\left(\mathbf{F}_{q}\right)$, and $c_{1}$ is the $(1,2)^{t h}$ entry of $C$, and $\vec{b}=\left(\begin{array}{l}b_{1} \\ b_{2}\end{array}\right)$, and $\vec{d}=\left(\begin{array}{l}d_{1} \\ d_{2}\end{array}\right)$ Let $B=\left(\begin{array}{ccc}C & \vec{b} & \vec{d} \\ & 0 & c_{1} \\ & 1 & a \\ & & 1\end{array}\right)$, and $B^{\prime}=\left(\begin{array}{ccc}C^{\prime} & \overrightarrow{b^{\prime}} & \overrightarrow{d^{\prime}} \\ & 0 & c_{1}^{\prime} \\ & 1 & a^{\prime} \\ & & 1\end{array}\right)$ be a conjugate of $B$ by $X=$ $\left(\begin{array}{ccc}Z & \vec{y} & \vec{w} \\ & 0 & z_{1} \\ & 1 & x \\ & 1\end{array}\right)$ Then from $X B=B^{\prime} X$, we have $Z C=C^{\prime} Z$. Thus, we can $C$ to be a conjugacy class representative in $U T_{3}\left(\mathbf{F}_{q}\right)$, and $Z \in Z_{U T_{3}\left(\mathbf{F}_{q}\right)}(C)$, and we also have $a^{\prime}=a$. With this, we have the following equations

$$
\begin{aligned}
& Z\left(\begin{array}{c}
\vec{b} \\
0
\end{array}\right)=\left(C-I_{3}\right)\left(\begin{array}{c}
\vec{y} \\
0
\end{array}\right)+\left(\begin{array}{c}
\overrightarrow{b^{\prime}} \\
0
\end{array}\right) \\
& Z\left(\begin{array}{c}
\vec{d} \\
c_{1}
\end{array}\right)+\left(\begin{array}{c}
a \vec{y} \\
0
\end{array}\right)=\left(C-I_{3}\right)\left(\begin{array}{c}
\vec{w} \\
z_{1}
\end{array}\right)+\left(\begin{array}{c}
x \vec{b} \\
0
\end{array}\right)+\left(\begin{array}{c}
\overrightarrow{d^{\prime}} \\
c_{1}
\end{array}\right)
\end{aligned}
$$


We first look at the case $\vec{b}=\overrightarrow{0}$ :

When $a=0$ : Here, Equation 7.13 becomes $\left(C-I_{3}\right)\left(\begin{array}{l}\vec{y} \\ 0\end{array}\right)=\left(\begin{array}{l}0 \\ 0 \\ 0\end{array}\right)$.

When $C=I_{3}$ : Here Equation 7.13 becomes void, and Equation 7.14 becomes $\left(\begin{array}{c}d_{1}+z_{1} d_{2} \\ d_{2} \\ 0\end{array}\right)=\left(\begin{array}{c}d_{1}^{\prime} \\ d_{2}^{\prime} \\ 0\end{array}\right)$.

When $d_{2}=0$, we have $d_{1}^{\prime}=d_{1}$. Thus $B=\left(\begin{array}{lllll}1 & & & & d_{1} \\ & 1 & & & \\ & & 1 & & \\ & & 1 & \\ & & & 1\end{array}\right)$, and $Z_{U T_{5}\left(\mathbf{F}_{q}\right)}(A, B)=$ $Z_{U T_{5}\left(\mathbf{F}_{q}\right)}(A)$. So $(A, B)$ is a branch of type $B_{2}$, and there are $q$ such branches.

When $d_{2} \neq 0$, choose $z_{1}$ so that $d_{1}^{\prime}=0$. Then $B$ is reduced to $\left(\begin{array}{ccccc}1 & & & & \\ & 1 & & & d_{2} \\ & 1 & & \\ & & 1 & \\ & & & & 1\end{array}\right)$.

Hence $Z_{U T_{5}\left(\mathbf{F}_{q}\right)}(A, B)=\left\{\left(\begin{array}{ccccc}1 & & z_{2} & y_{1} & w_{1} \\ & 1 & z_{3} & y_{2} & w_{2} \\ & 1 & & \\ & & 1 & x \\ & & & 1\end{array}\right)\right\}$, which is the centralizer of a matrix of type $A_{4}$. So $(A, B)$ is of type $A_{4}$, and there are $(q-1)$ such branches.

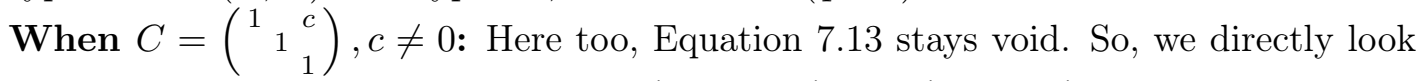
at Equation 17.14, which boils down to: $\left(\begin{array}{c}d_{1}+z_{1} d_{2} \\ d_{2} \\ 0\end{array}\right)=\left(\begin{array}{c}d_{1}^{\prime}+c z_{1} \\ d_{2}^{\prime} \\ 0\end{array}\right)$. We have $d_{2}^{\prime}=d_{2}$. We look at two cases here:

When $d_{2}=c$, we get $d_{1}^{\prime}=d_{1}$, and thus $B$ is reduced to $\left(\begin{array}{ccccc}1 & & c & & d_{1} \\ & 1 & & & \\ & & 1 & & \\ & & 1 & \\ & & & 1\end{array}\right)$, and $Z_{U T_{5}\left(\mathbf{F}_{q}\right)}(A, B)=Z_{U T_{5}\left(\mathbf{F}_{q}\right)}(A) .(A, B)$ is of type $B_{2}$, and there are $q(q-1)$ such branches. 
When $d_{2} \neq c$, choose $z_{1}$ such that $d_{1}^{\prime}=0$. Thus $B$ boils down to $\left(\begin{array}{ccccc}1 & & c & & \\ & 1 & & & d_{2} \\ & 1 & & \\ & & 1 & \\ & & & & 1\end{array}\right)$, and $Z_{U T_{5}\left(\mathbf{F}_{q}\right)}(A, B)=\left\{\left(\begin{array}{ccccc}1 & & z_{2} & y_{1} & w_{1} \\ & 1 & z_{3} & y_{2} & w_{2} \\ & & 1 & & \\ & & & 1 & x \\ & & & & 1\end{array}\right)\right\} .(A, B)$ is of type $A_{4}$, and there are $(q-1)^{2}$ such branches.

When $C=\left(\begin{array}{ccc}1 & c & \\ & 1 & \\ & & 1\end{array}\right), c \neq 0$ : Here $Z=\left(\begin{array}{ccc}1 & z_{1} & z_{2} \\ & 1 & \\ & & 1\end{array}\right)$. From Equation 7.13 we have $\left(\begin{array}{ccc}0 & c & 0 \\ & & \end{array}\right)\left(\begin{array}{l}y_{1} \\ y_{2}\end{array}\right)=\overrightarrow{0}$. We have $c y_{2}=0$, thus $y_{2}=0$. Equation 7.14 becomes: $\left(\begin{array}{c}d_{1}+z_{1} d_{2}+z_{2} c \\ d_{2} \\ c\end{array}\right)=\left(\begin{array}{c}d_{1}^{\prime}+c w_{2} \\ d_{2}^{\prime} \\ c\end{array}\right)$. Choose $w_{2}$ such that $d_{1}^{\prime}=0$. Thus $B$ is reduced to $\left(\begin{array}{ccccc}1 & c & & & \\ & 1 & & & d_{2} \\ & & 1 & & c \\ & & 1 & \\ & & & 1\end{array}\right)$, and $Z_{U T_{5}\left(\mathbf{F}_{q}\right)}(A, B)=\left\{\left(\begin{array}{ccccc}1 & z_{1} & z_{2} & y_{1} & w_{1} \\ & 1 & & & z_{2}+\frac{d_{2}}{c} z_{1} \\ & 1 & & z_{1} \\ & & 1 & x \\ & & & 1\end{array}\right)\right\} .(A, B)$ is of type $B_{6}$, and there are $(q-1) q$ such branches.

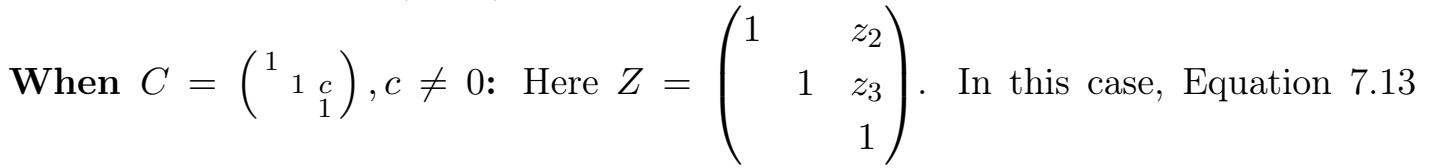
stays void. So we directly jump to Equation 7.14. We have $\left(\begin{array}{l}d_{1} \\ d_{2}\end{array}\right)=\left(\begin{array}{l}d_{1}^{\prime} \\ d_{2}^{\prime}\end{array}\right)$. So we have

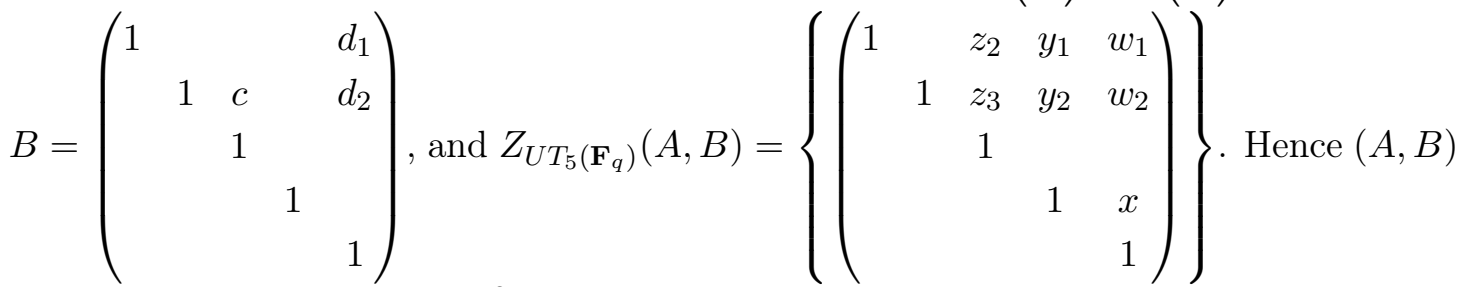
is of type $A_{4}$, and there are $q^{2}(q-1)$ such branches. 


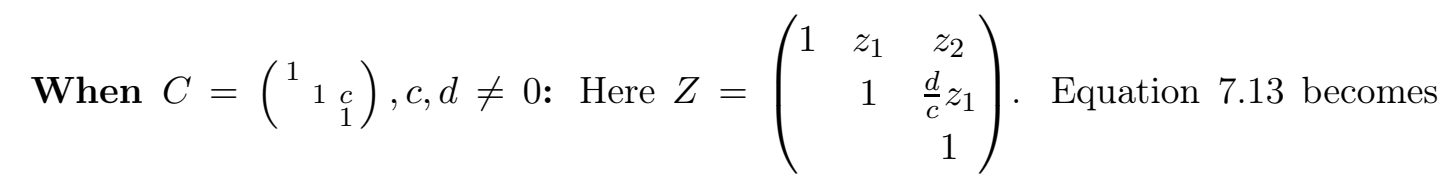
$\left(\begin{array}{c}c y_{2} \\ 0 \\ 0\end{array}\right)=\overrightarrow{0}$. Thus $y_{2}=0$. From Equation 7.14 we have $\left(\begin{array}{c}d_{1}+z_{1} d_{2}+c z_{2} \\ d_{2}+z_{1} d \\ c\end{array}\right)=$ $\left(\begin{array}{l}d_{1}^{\prime}+c w_{2} \\ d_{2}^{\prime}+d z_{1}\end{array}\right)$. Hence $d_{2}^{\prime}=d_{2}$, and choose $w_{2}$ such that $d_{1}^{\prime}=0$. So $B$ boils down to $\left(\begin{array}{ccccc}1 & c & & & \\ & 1 & d & & d_{2} \\ & & 1 & & c \\ & & & 1 & \\ & & & & 1\end{array}\right)$, and $Z_{U T_{5}\left(\mathbf{F}_{q}\right)}(A, B)=\left\{\left(\begin{array}{ccccc}1 & z_{1} & z_{2} & y_{1} & w_{1} \\ & 1 & \frac{d}{c} z_{1} & & z_{2}+\frac{d_{2}}{c} z_{1} \\ & 1 & & z_{1} \\ & & 1 & x \\ & & & 1\end{array}\right)\right\} .(A, B)$ is of type $B_{6}$ and there are $(q-1)^{2} q$ such branches.

bfseries $a \neq 0$ : We are still dealing with $\vec{b}=\overrightarrow{0}$ here. So Equation 7.13 becomes $\left(C-I_{3}\right)\left(\begin{array}{c}y_{1} \\ y_{2} \\ 0\end{array}\right)=\left(\begin{array}{l}0 \\ 0 \\ 0\end{array}\right)$. And Equation 7.14 becomes: $Z\left(\begin{array}{c}d_{1} \\ d_{2} \\ c_{1}\end{array}\right)+a\left(\begin{array}{c}y_{1} \\ y_{2} \\ 0\end{array}\right)=\left(\begin{array}{c}d_{1}^{\prime} \\ d_{2}^{\prime} \\ c_{1}\end{array}\right)+$ $\left(C-I_{3}\right)\left(\begin{array}{l}w_{1} \\ w_{2} \\ z_{1}\end{array}\right)$

When $C=I_{3}$ : Equation 7.13 becomes void, and from Equation 7.14, we have $\left(\begin{array}{c}d_{1}+z_{1} d_{2}+a y_{1} \\ d_{2}+a y_{2} \\ 0\end{array}\right)=\left(\begin{array}{c}d_{1}^{\prime} \\ d_{2}^{\prime} \\ 0\end{array}\right)$. Choose $y_{2}$ and $y_{1}$ such that, $d_{2}^{\prime}$ and $d_{1}^{\prime}$ become 0 . Hence,

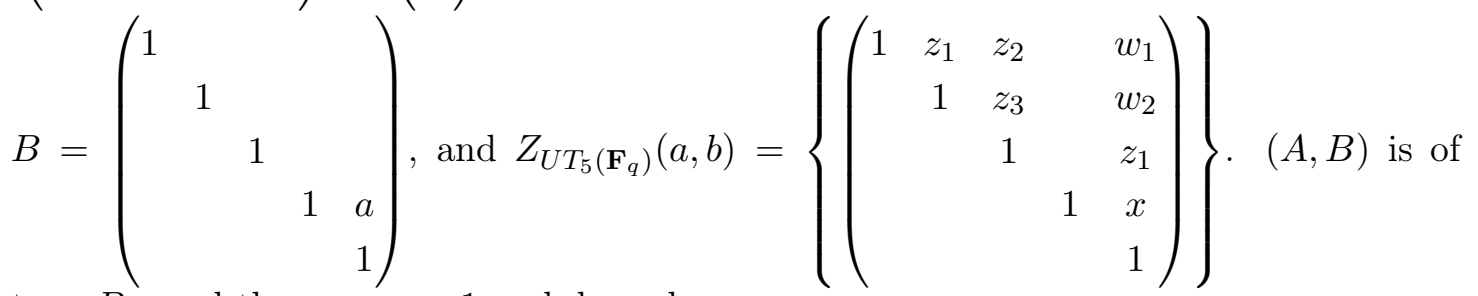
type $B_{4}$, and there are $q-1$ such branches.

When $C=\left(\begin{array}{ccc}1 & c & c \\ & 1 & \\ & & 1\end{array}\right), c \neq 0$ : Here also, Equation 7.13 remains void. Equation 7.14 becomes: $\left(\begin{array}{c}d_{1}+z_{2} d_{2}+a y_{1} \\ d_{2}+a y_{2} \\ 0\end{array}\right)=\left(\begin{array}{c}d_{1}^{\prime}+c z_{1} \\ d_{2}^{\prime} \\ 0\end{array}\right)$. Choose $y_{2}$ and $y_{1}$ so that $d_{2}^{\prime}=d_{1}^{\prime}=0$. Thus, 
$B$ is reduced to $\left(\begin{array}{ccccc}1 & & c & & \\ & 1 & & & \\ & & 1 & & \\ & & & 1 & a \\ & & & & 1\end{array}\right)$, and $Z_{U T_{5}\left(\mathbf{F}_{q}\right)}(A, B)=\left\{\left(\begin{array}{ccccc}1 & z_{1} & z_{2} & \frac{c}{a} z_{1} & w_{1} \\ & 1 & z_{3} & & w_{2} \\ & & 1 & & z_{1} \\ & & & 1 & x \\ & & & & 1\end{array}\right)\right\}$.

$(A, B)$ is of type $B_{4}$, and there are $(q-1)^{2}$ such branches.

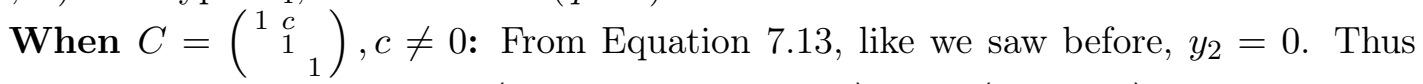
Equation 7.14 boils down to $\left(\begin{array}{c}d_{1}+z_{1} d_{2}+z_{1} c+a y_{1} \\ d_{2} \\ c\end{array}\right)=\left(\begin{array}{c}d_{1}^{\prime}+c w_{2} \\ d_{2} \\ c\end{array}\right)$. We see that $d_{2}^{\prime}=d_{2}$. Choose $w_{2}$ such that $d_{1}^{\prime}=0$. So $B$ is reduced to $\left(\begin{array}{ccccc}1 & c & & & \\ & 1 & & & d_{2} \\ & 1 & & c \\ & & 1 & a_{1} \\ & & & 1\end{array}\right)$, and $Z_{U T_{5}\left(\mathbf{F}_{q}\right)}(A, B)=\left\{\left(\begin{array}{ccccc}1 & z_{1} & z_{2} & y_{1} & w_{1} \\ & 1 & & & z_{2}+\frac{d_{2}}{c} z_{1}+\frac{a}{c} y_{1} \\ & & 1 & & z_{1} \\ & & & 1 & x \\ & & & & \end{array}\right)\right\} .(A, B)$ is of type $B_{6}$, and there are $(q-1)^{2} q$ such branches.

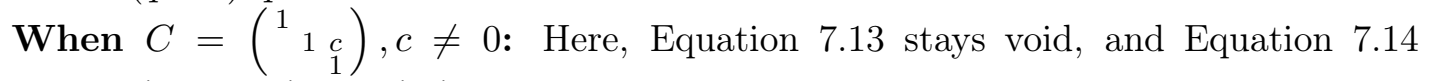
becomes $\left(\begin{array}{c}d_{1}+a y_{1} \\ d_{2}+a y_{2} \\ 0\end{array}\right)=\left(\begin{array}{c}d_{1}^{\prime} \\ d_{2}^{\prime} \\ 0\end{array}\right)$. Choose $y_{1}, y_{2}$ such that $d_{1}^{\prime}=d_{2}^{\prime}=0$. SO $B$ is reduced to $\left(\begin{array}{ccccc}1 & & & & \\ & 1 & c & & \\ & & 1 & & \\ & & & 1 & a \\ & & & & 1\end{array}\right)$, and $Z_{U T_{5}\left(\mathbf{F}_{q}\right)}(A, B)=\left\{\left(\begin{array}{ccccc}1 & & z_{2} & & w_{1} \\ & 1 & z_{3} & & w_{2} \\ & & 1 & & \\ & & 1 & x \\ & & & 1\end{array}\right)\right\} .(A, B)$ is therefore of type $R_{2}$, and there are $(q-1)^{2}$ such branches.

When $C=\left(\begin{array}{ccc}1 & c & \\ & 1 & d \\ & & 1\end{array}\right), c, d \neq 0$ : Here, like earlier, from Equation [7.13, we get $y_{2}=0$. Hence Equation 7.14 boils down to $\left(\begin{array}{c}d_{1}+z_{1} d_{2}+z_{2} c+a y_{1} \\ d_{2}+z_{1} d \\ c\end{array}\right)=\left(\begin{array}{c}d_{1}^{\prime}+c w_{2} \\ d_{2}^{\prime}+d z_{1} \\ c\end{array}\right)$. This leaves us with $d_{2}^{\prime}=d_{2}$, and choose $y_{1}$ such that $d_{1}^{\prime}=0$. So we have $B$ boiling down to 


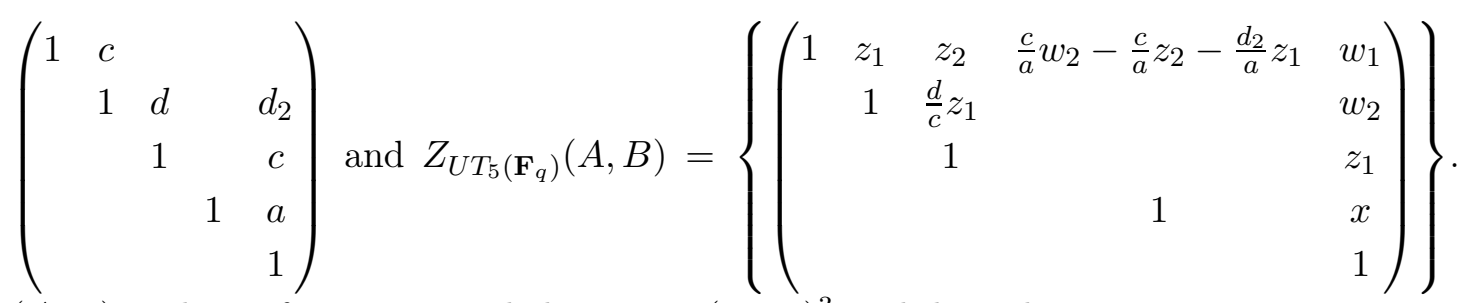

$(A, B)$ is thus, of type $B_{6}$, and there are $q(q-1)^{3}$ such branches.

Now we look at what happens, when $\vec{b} \neq \overrightarrow{0}$.

$$
\text { When } C=I_{3} \text { : Subcase } a=0 \text { : From Equation } 7.13 \text { we have }\left(\begin{array}{c}
b_{1}+z_{1} b_{2} \\
b_{2} \\
0
\end{array}\right)=\left(\begin{array}{c}
b_{1}^{\prime} \\
b_{2}^{\prime} \\
0
\end{array}\right) \text {. }
$$

When $b_{2} \neq 0$, we choose $z_{1}$ such that $b_{1}^{\prime}=0$. Thus replacing $b_{1}$ by 0 in the above equation, we obtain $z_{1}=0$. Hence, Equation 7.14 boils down to $\left(\begin{array}{c}d_{1} \\ d_{2} \\ 0\end{array}\right)=\left(\begin{array}{c}d_{1}^{\prime} \\ d_{2}^{\prime}+x b_{2} \\ 0\end{array}\right)$.

We have $d_{1}^{\prime}=d_{1}$. Choose $x$ such that $d_{2}^{\prime}=0$. So $B$ boils down to $\left(\begin{array}{lllll}1 & & & d_{1} \\ & & b_{2} & \\ & 1 & & \\ & & 1 & \\ & & & 1\end{array}\right)$,

and $Z_{U T_{5}\left(\mathbf{F}_{q}\right)}(A, B)=\left\{\left(\begin{array}{ccccc}1 & & z_{2} & y_{1} & w_{1} \\ & 1 & z_{3} & y_{2} & w_{2} \\ & & 1 & & \\ & & & 1 & \\ & & & & 1\end{array}\right)\right\} .(A, B)$ is of type $R_{1}$, and there are $q(q-1)$ such branches.

When $b_{2}=0$, we have to look at $b_{1} \neq 0$, and we have $b_{1}^{\prime}=b_{1}$. Equation 7.14 becomes $\left(\begin{array}{c}d_{1}+z_{1} d_{2} \\ d_{2} \\ 0\end{array}\right)=\left(\begin{array}{c}d_{1}^{\prime}+x b_{1} \\ d_{2}^{\prime} \\ 0\end{array}\right)$. So $d_{2}^{\prime}=d_{2}$, and choose $x$ such that $d_{1}^{\prime}=0$. Hence $B=\left(\begin{array}{ccccc}1 & & & b_{1} & \\ & 1 & & & d_{2} \\ & & 1 & & \\ & & & 1 & \\ & & & & 1\end{array}\right)$, and $Z_{U T_{5}\left(\mathbf{F}_{q}\right)}(A, B)=\left\{\left(\begin{array}{ccccc}1 & z_{1} & z_{2} & y_{1} & w_{1} \\ & 1 & z_{3} & y_{2} & w_{2} \\ & 1 & & z_{1} \\ & & 1 & \frac{d_{2}}{b_{1}} z_{1} \\ & & & & 1\end{array}\right)\right\}$. Thus $(A, B)$ is of the new type $U N T_{2}$, and there are $(q-1) q$ such branches. 
Subcase $a \neq 0$, and $b_{2} \neq 0$ : In Equation [7.13, we choose $z_{1}$ to get rid of $b_{1}^{\prime}$, and like before $z_{1}=0$. Equation 7.14 becomes: $\left(\begin{array}{c}d_{1}+a y_{1} \\ d_{2}+a y_{2} \\ 0\end{array}\right)=\left(\begin{array}{c}d_{1}^{\prime} \\ d_{2}^{\prime}+x b_{2} \\ 0\end{array}\right)$. Choose $y_{1}$ such that $d_{1}^{\prime}=0$, and $x$ such that $d_{2}^{\prime}=0$. So $B=\left(\begin{array}{ccccc}1 & & & & \\ & 1 & & b_{2} & \\ & 1 & & \\ & & 1 & a \\ & & & 1\end{array}\right)$, and $Z_{U T_{5}\left(\mathbf{F}_{q}\right)}(A, B)=$ $\left\{\left(\begin{array}{ccccc}1 & & z_{2} & & w_{1} \\ & 1 & z_{3} & y_{2} & w_{2} \\ & 1 & & \\ & & 1 & \frac{a}{b_{2}} y_{2} \\ & & & & 1\end{array}\right)\right\}$. Thus $(A, B)$ is of type $R_{2}$, and there are $(q-1)^{2}$ such branches.

Subcase $a \neq 0$ and $b_{2}=0$. Here we have $b_{1}^{\prime}=b_{1} \neq 0$. From Equation 7.14 we have $\left(\begin{array}{c}d_{1}+z_{1} d_{2}+a y_{1} \\ d_{2}+a y_{2} \\ 0\end{array}\right)=\left(\begin{array}{c}d_{1}^{\prime}+b_{1} x \\ d_{2}^{\prime} \\ 0\end{array}\right)$. Choose $y_{2}$ such that $d_{2}^{\prime}=0$, and $x$ such that $d_{1}^{\prime}=0$. Thus $B$ is reduced to $\left(\begin{array}{lllll}1 & & & b_{1} & \\ & 1 & & & \\ & 1 & & \\ & & 1 & a \\ & & & 1\end{array}\right)$, and $Z_{U T_{5}\left(\mathbf{F}_{q}\right)}(A, B)=$ $\left\{\left(\begin{array}{ccccc}1 & z_{1} & z_{2} & y_{1} & w_{1} \\ & 1 & z_{3} & & w_{2} \\ & & 1 & & z_{1} \\ & & & 1 & \frac{a}{b_{1}} y_{1} \\ & & & & 1\end{array}\right)\right\}$. $(A, B)$ is of new type $U N T_{1}$, and there are $(q-1)^{2}$ such branches.

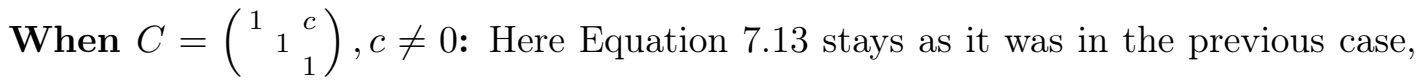
i.e., $\left(\begin{array}{c}b_{1}+z_{1} b_{2} \\ b_{2} \\ 0\end{array}\right)=\left(\begin{array}{c}b_{1}^{\prime} \\ b_{2}^{\prime} \\ 0\end{array}\right)$. 
When $b_{2} \neq 0$, choose $z_{1}$ so that $b_{1}^{\prime}=0$, and on replacing $b_{1}$ with $b_{1}^{\prime}=0$ in the above equation, we get $z_{1}=0$. Hence, Equation 7.14 becomes: $\left(\begin{array}{c}d_{1}+a y_{1} \\ d_{2}+a y_{2} \\ 0\end{array}\right)=\left(\begin{array}{c}d_{1}^{\prime} \\ d_{2}^{\prime}+x b_{2} \\ 0\end{array}\right)$. We can choose $x$ such that $d_{2}^{\prime}=0$.

Subcase $a=0$. We have in this $d_{1}^{\prime}=d_{1}$. $B$ reduces to $\left(\begin{array}{ccccc}1 & & c & & d_{1} \\ & 1 & & b_{2} & \\ & 1 & & \\ & & 1 & \\ & & & 1\end{array}\right)$, with
$Z_{U T_{5}\left(\mathbf{F}_{q}\right)}(A, B)=\left\{\left(\begin{array}{ccccc}1 & & z_{2} & y_{1} & w_{1} \\ & 1 & z_{3} & y_{2} & w_{2} \\ & & 1 & & \\ & & 1 & \\ & & & & \\ & & & & \end{array}\right)\right\}$. So, $(A, B)$ is of type $R_{1}$, and there are $q(q-1)^{2}$ such branches.

Subcase $a \neq 0$. Here, in addition to getting rid of $d_{2}^{\prime}$, we choose $y_{1}$ such that $d_{1}^{\prime}=0$. So, $B$ reduces to $\left(\begin{array}{ccccc}1 & & c & & \\ & 1 & & b_{2} & \\ & & 1 & & \\ & & & 1 & a \\ & & & & 1\end{array}\right)$, with $Z_{U T_{5}\left(\mathbf{F}_{q}\right)}(A, B)=\left\{\left(\begin{array}{ccccc}1 & & z_{2} & & w_{1} \\ & 1 & z_{3} & y_{2} & w_{2} \\ & & 1 & & \\ & & 1 & \frac{a}{b_{2}} y_{2} \\ & & & & 1\end{array}\right)\right\}$. So $(A, B)$ is of type $R_{2}$, and there are $(q-1)^{3}$ such branches.

When $b_{2}=0$, here $b_{1}^{\prime}=b_{1} \neq 0$. Equation 7.14 becomes $\left(\begin{array}{c}d_{1}+z_{1} d_{2}+a y_{1} \\ d_{2} \\ 0\end{array}\right)=$ $\left(\begin{array}{c}d_{1}^{\prime}+b_{1} x+c z_{1} \\ d_{2}^{\prime} \\ 0\end{array}\right)$. Choose $x$ so that $d_{1}^{\prime}=0$ 


$$
\begin{aligned}
& \text { Subcase } a=0 \text {. Here } d_{2}^{\prime}=d_{2} \text {, and } B \text { thus reduces to }\left(\begin{array}{ccccc}
1 & & c & b_{1} & \\
& 1 & & & d_{2} \\
& & 1 & & \\
& & 1 & \\
& & & & 1
\end{array}\right) \text {, with } \\
& Z_{U T_{5}\left(\mathbf{F}_{q}\right)}(A, B)=\left\{\left(\begin{array}{ccccc}
1 & z_{1} & z_{2} & y_{1} & w_{1} \\
& 1 & z_{3} & y_{2} & w_{2} \\
& & 1 & & z_{1} \\
& & & 1 & \frac{d_{2}-c}{b_{1}} z_{1} \\
& & & & 1
\end{array}\right)\right\} \text {. Hence }(A, B) \text { is of the new type } U N T_{2},
\end{aligned}
$$
and there are $(q-1)^{2} q$ such branches.

Subcase $a \neq 0$. Here, choose $y_{2}$ such that $d_{2}^{\prime}=0$. Hence $B$ is reduced to $\left(\begin{array}{cccccc}1 & & c & b_{1} & \\ & 1 & & & \\ & 1 & & \\ & & & 1 & a \\ & & & & 1\end{array}\right)$, with $Z_{U T_{5}\left(\mathbf{F}_{q}\right)}(A, B)=\left\{\left(\begin{array}{ccccc}1 & z_{1} & z_{2} & y_{1} & w_{1} \\ & 1 & z_{3} & & w_{2} \\ & & 1 & & z_{1} \\ & & & 1 & \frac{a}{b_{1}} y_{1}-\frac{c}{b_{1}} z_{1} \\ & & & & 1\end{array}\right)\right\}$. Hence $(A, B)$ is of type $U N T_{1}$, and there are $(q-1)^{3}$ such branches.

$$
\text { When } C=\left(\begin{array}{ccc}
1 & c & \\
& 1 & \\
& & 1
\end{array}\right), c \neq 0 \text { : Here Equation } 7.13 \text { becomes }\left(\begin{array}{c}
b_{1}+z_{1} b_{2} \\
b_{2} \\
0
\end{array}\right)=\left(\begin{array}{c}
b_{1}^{\prime}+c y_{2} \\
b_{2}^{\prime} \\
0
\end{array}\right) \text {. }
$$

Choose $y_{2}$ such that $b_{1}^{\prime}=0$. We have $b_{2}^{\prime}=b_{2} \neq 0$. On replacing $b_{1}$ with 0 in the above equation, we get $y_{2}=\frac{b_{2}}{c} z_{1}$. Equation 7.14 thus becomes $\left(\begin{array}{c}d_{1}+z_{1} d_{2}+c z_{2}+a y_{1} \\ d_{2}+\frac{a b_{2}}{c} z_{1} \\ c\end{array}\right)=$ $\left(\begin{array}{c}d_{1}^{\prime}+c w_{2} \\ d_{2}^{\prime}+x b_{2} \\ \\ c\end{array}\right)$. Choose $w_{2}$ such that $d_{1}^{\prime}=0$, and $x$ such that $d_{2}^{\prime}=0$. Hence $B$ is reduced to
$\left(\begin{array}{ccccc}1 & c & & & \\ & 1 & & b_{2} & \\ & 1 & & c \\ & & 1 & a \\ & & & 1\end{array}\right)$, with $Z_{U T_{5}\left(\mathbf{F}_{q}\right)}(A, B)=\left\{\left(\begin{array}{ccccc}1 & z_{1} & z_{2} & y_{1} & w_{1} \\ & 1 & & \frac{b_{2}}{c} z_{1} & \frac{a}{c} y_{1}+z_{2} \\ & 1 & & z_{1} \\ & & 1 & \frac{a}{c} z_{1} \\ & & & 1\end{array}\right)\right\} .(A, B)$ is of type $R_{3}$, and there are $(q-1)^{2} q$ such branches. 


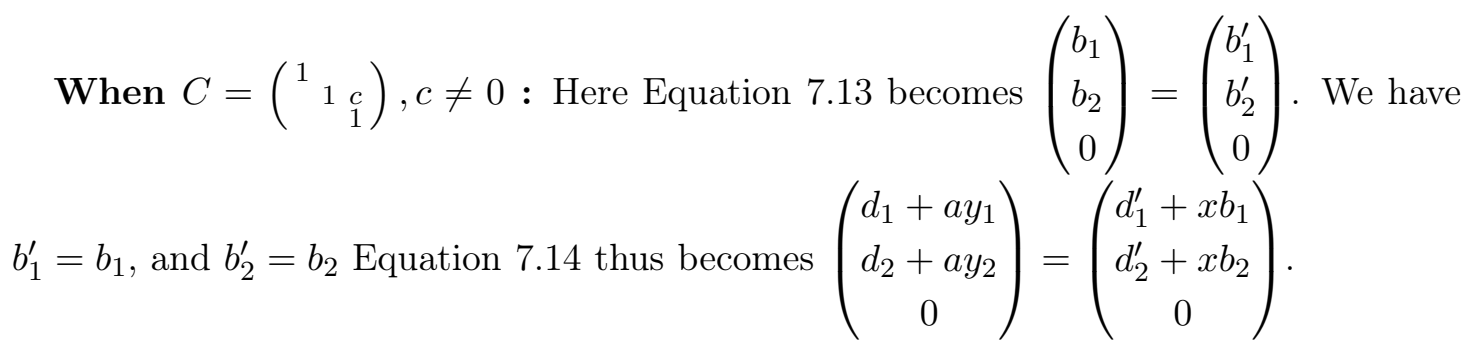

Subcase $a=0$ : When $b_{1} \neq 0$, choose $x$ such that $d_{1}^{\prime}=0$. Thus, on replacing $d_{1}$ with $d_{1}^{\prime}=0$, we get $x=0$, and thus $d_{2}^{\prime}=d_{2}$. Hence $B$ is reduced to $\left(\begin{array}{ccccc}1 & & & b_{1} & \\ & 1 & c & b_{2} & d_{2} \\ & 1 & & \\ & & 1 & \\ & & & & 1\end{array}\right)$, with $Z_{U T_{5}\left(\mathbf{F}_{q}\right)}(A, B)=\left\{\left(\begin{array}{ccccc}1 & & z_{2} & y_{1} & w_{1} \\ & 1 & z_{3} & y_{2} & w_{2} \\ & & 1 & & \\ & & & 1 & \\ & & & & 1\end{array}\right)\right\} .(A, B)$ is of type $R_{1}$, and there are $(q-1)^{2} q^{2}$ such branches.

When $b_{1}=0$, we work with $b_{2} \neq 0$. Choose $x$ such that $d_{2}^{\prime}=0$, and with this on replacing $d_{2}$ with $d_{2}^{\prime}=0$, we have $x=0$, which leaves us with $d_{1}^{\prime}=d_{1} . B$ is reduced

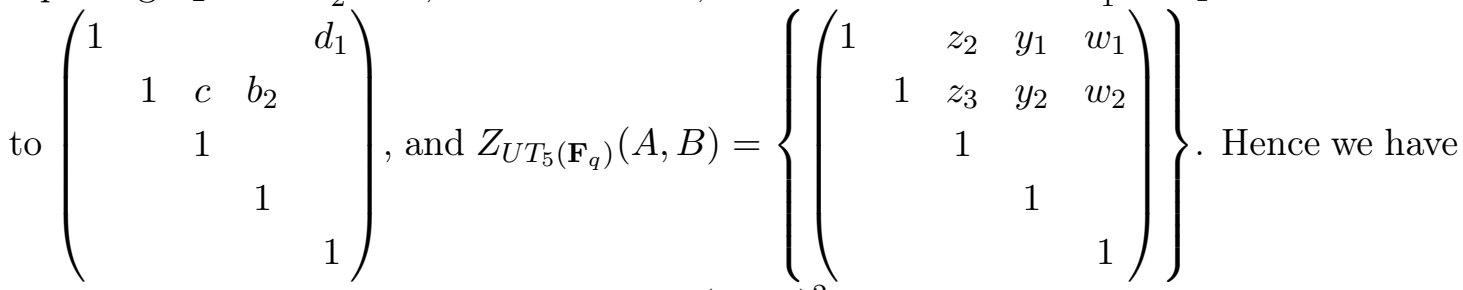
another branch of type $R_{1}$, and these are $(q-1)^{2} q$ in number.

Subcase $a \neq 0$. We just choose $y_{1}, y_{2}$ such that $d_{1}^{\prime}=d_{2}^{\prime}=0$. Here $\left(b_{1}, b_{2}\right) \neq(0,0)$.

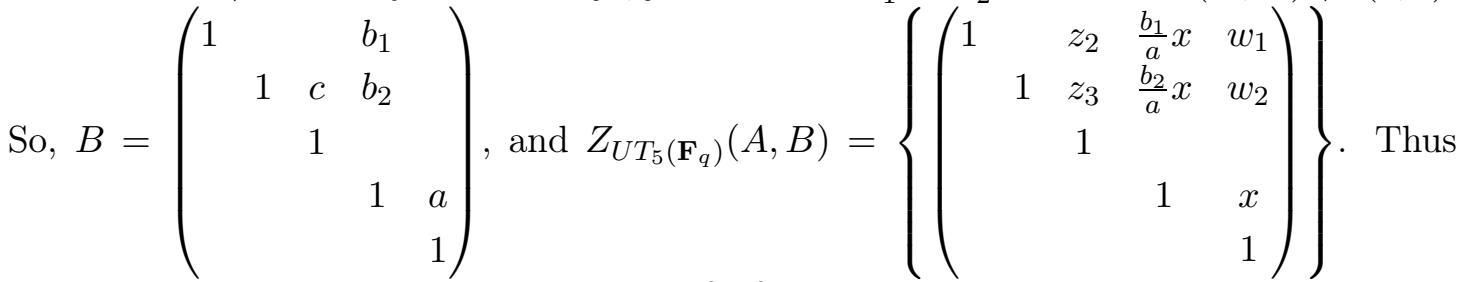

$(A, B)$ is of type $R_{2}$, and there are $(q-1)^{2}\left(q^{2}-1\right)$ such branches $\left(\right.$ as $\left.\left(b_{1}, b_{2}\right) \neq(0,0)\right)$.

When $C=\left(\begin{array}{ccc}1 & c & \\ & 1 & d \\ & & 1\end{array}\right), c, d \neq 0$ : Here Equation 7.13 becomes $\left(\begin{array}{c}b_{1}+z_{1} b_{2} \\ b_{2} \\ 0\end{array}\right)=\left(\begin{array}{c}b_{1}^{\prime}+c y_{2} \\ b_{2}^{\prime} \\ 0\end{array}\right)$.

Choose $y_{2}$ such that $b_{1}^{\prime}=0$. We have $b_{2}^{\prime}=b_{2} \neq 0$. On replacing $b_{1}$ with 0 in the above 
equation, we get $y_{2}=\frac{b_{2}}{c} z_{1}$. Equation 7.14 thus becomes $\left(\begin{array}{c}d_{1}+z_{1} d_{2}+c z_{2}+a y_{1} \\ d_{2}+\frac{a b_{2}}{c} z_{1}+d z_{1} \\ c\end{array}\right)=$ $\left(\begin{array}{c}d_{1}^{\prime}+c w_{2} \\ d_{2}^{\prime}+x b_{2}+d z_{1} \\ c\end{array}\right)$. Choose $w_{2}$ such that $d_{1}^{\prime}=0$, and $x$ such that $d_{2}^{\prime}=0$. Hence $B$ is reduced to $\left(\begin{array}{ccccc}1 & c & & & \\ & 1 & d & b_{2} & \\ & & 1 & & c \\ & & & 1 & a \\ & & & & 1\end{array}\right)$, with $Z_{U T_{5}\left(\mathbf{F}_{q}\right)}(A, B)=\left\{\left(\begin{array}{ccccc}1 & z_{1} & z_{2} & y_{1} & w_{1} \\ & 1 & \frac{d}{c} z_{1} & \frac{b_{2}}{c} z_{1} & \frac{a}{c} y_{1}+z_{2} \\ & 1 & & z_{1} \\ & & 1 & \frac{a}{c} z_{1} \\ & & & 1\end{array}\right)\right\}$. $(A, B)$ is of type $R_{3}$, and there are $(q-1)^{3} q$ such branches.

Proposition 7.9. An upper unitriangular matrix of type $B_{3}$ has $q^{3}$ branches of type $B_{3}$, $q^{2}\left(q^{2}+q+1\right)(q-1)$ branches of regular type $R_{1}$, and $q^{3}(q-1)$ branches of regular type $R_{3}$.

Proof. Let $A=\left(\begin{array}{ccccc}1 & & a & & \\ & 1 & & b & \\ & & 1 & & \\ & & & 1 & \\ & & & & 1\end{array}\right), a, b \neq 0$ a matrix of type $B_{3}$. The centralizer

$Z_{U T_{5}}(A)$ of $A$ is $\left\{\left(\begin{array}{ccccc}1 & x_{0} & x_{1} & x_{2} & x_{3} \\ & 1 & y_{0} & y_{1} & y_{2} \\ & & 1 & \lambda x_{0} & \\ & & & 1 & \\ & & & & 1\end{array}\right) \mid \lambda=\frac{b}{a}, x_{i}, y_{i} \in \mathbf{F}_{q}\right\}$. Let $X=\left(\begin{array}{ccccc}1 & x_{0} & x_{1} & x_{2} & x_{3} \\ & 1 & y_{0} & y_{1} & y_{2} \\ & 1 & \lambda x_{0} & \\ & & 1 & \\ & & & & 1\end{array}\right)$

be an element of $Z_{U T_{5}\left(\mathbf{F}_{q}\right)}(A)$. Let $B=\left(\begin{array}{ccccc}1 & a_{0} & a_{1} & a_{2} & a_{3} \\ & 1 & b_{0} & b_{1} & b_{2} \\ & & 1 & \lambda a_{0} & \\ & & & 1 & \\ & & & & 1\end{array}\right)$, and $B^{\prime}=\left(\begin{array}{ccccc}1 & a_{0}^{\prime} & a_{1}^{\prime} & a_{2}^{\prime} & a_{3}^{\prime} \\ & 1 & b_{0}^{\prime} & b_{1}^{\prime} & b_{2}^{\prime} \\ & 1 & \lambda a_{0}^{\prime} & \\ & & & 1 & \\ & & & & 1\end{array}\right)$

be a conjugate of $B$ by $X$. Thus equating $X B=B^{\prime} X$ gives us $a_{0}=a_{0}^{\prime}, b_{0}=b_{0}^{\prime}, b_{2}=b_{2}^{\prime}$, and the following equations:

$$
\begin{aligned}
a_{1}+b_{0} x_{0} & =a_{0}^{\prime} y_{0}+a_{1}^{\prime} \\
a_{2}+x_{0} b_{1}+\lambda x_{1} a_{0} & =a_{0}^{\prime} y_{1}+\lambda x_{0} a_{1}^{\prime}+a_{2}^{\prime} \\
a_{3}+x_{0} b_{2} & =a_{3}^{\prime}+a_{0}^{\prime} y_{2} \\
b_{1}+\lambda a_{0} y_{0} & =\lambda x_{0} b_{0}^{\prime}+b_{1}^{\prime}
\end{aligned}
$$


We look at three cases, the first case is when $\lambda a_{1}=b_{1}$ and $\left(a_{0}, b_{0}, b_{2}\right)=\mathbf{0}$. The second case is when $\lambda a_{1} \neq b_{1}$ and $\left(a_{0}, b_{0}, b_{2}\right)=\mathbf{0}$. The third case is when $\left(a_{0}, b_{0}, b_{2}\right) \neq \mathbf{0}$.

Case: $\lambda a_{1}=b_{1}$ and $\left(a_{0}, b_{0}, b_{2}\right)=\mathbf{0}$. In this case, we get $a_{2}=a_{2}^{\prime}$. Therefore $Z_{U T_{5}\left(\mathbf{F}_{q}\right)}(A, B)=Z_{U T_{5}\left(\mathbf{F}_{q}\right)}(A)$. So $(A, B)$ is a branch of type $B_{3}$, and there are $q^{3}$ branches.

Case: $\lambda a_{1} \neq b_{1}$ and $\left(a_{0}, b_{0}, b_{2}\right)=\mathbf{0}$. In this case, we can choose $x_{0}$ in such a way that we get $a_{2}=0$. By routine check, we get $Z_{U T_{5}\left(\mathbf{F}_{q}\right)}(A, B)$ is a commutative group of size $q^{6}$. Thus $(A, B)$ is of regular type $R_{1}$, and there are $q^{2}(q-1)$ branches of this type.

Case: $\left(a_{0}, b_{0}, b_{2}\right) \neq \mathbf{0}$. We first consider that $a_{0} \neq 0$, then we can choose $y_{0}, y_{1}$ and $y_{2}$ in such a way that we get $a_{1}=a_{2}=a_{3}=0$ and $b_{1}=b_{1}^{\prime}$. By simple calculations, we get that $Z_{U T_{5}\left(\mathbf{F}_{q}\right)}(A, B)$ is a commutative group of size $q^{4}$. Thus $(A, B)$ is of regular type $R_{3}$, and there are $q^{3}(q-1)$ branches of this type.

Next we consider the case when $a_{0}=0$ and $b_{0} \neq 0$. Here we can choose $x_{0}$ in such a way that we get $a_{1}=0$. By routine check, we get $Z_{U T_{5}\left(\mathbf{F}_{q}\right)}(A, B)$ is commutative group of size $q^{6}$. Thus $(A, B)$ is of regular type $R_{1}$, and there are $q^{4}(q-1)$ branches of this type.

Finaly we consider the case when $a_{0}=b_{0}=0$ and $b_{2} \neq 0$, now we can choose $x_{0}$ in such a way that we get $a_{3}=0$. Again, we get $Z_{U T_{5}\left(\mathbf{F}_{q}\right)}(A, B)$ is commutative group of size $q^{6}$. Thus $(A, B)$ is of regular type $R_{1}$, and there are $q^{3}(q-1)$ branches of this type.

Therefore we get that a matrix of type $B_{3}$ has $q^{3}$ branches of type $B_{3}, q^{2}\left(q^{2}+q+1\right)(q-1)$ braches of regular type $R_{1}$, and $q^{3}(q-1)$ braches of regular type $R_{3}$.

Proposition 7.10. An upper unitriangular matrix of type $B_{4}$ has $q^{3}$ branches of type $B_{4}, q^{2}\left(q^{2}-1\right)$ branches of regular type $R_{2}$, and $q^{3}(q-1)$ branches of regular type $R_{3}$.

Proof. Let $A=\left(\begin{array}{ccccc}1 & a & & \\ & 1 & & b & \\ & & 1 & & \\ & & 1 & \\ & & & 1\end{array}\right), a, b \neq 0$ a matrix of type $B_{4}$. The centralizer
$Z_{U T_{5}}(A)$ of $A$ is $\left\{\left(\begin{array}{ccccc}1 & x_{0} & x_{1} & x_{2} & x_{3} \\ & 1 & & \lambda x_{0} & \\ & & 1 & z_{0} & z_{1} \\ & & & 1 & \\ & & & & 1\end{array}\right) \mid \lambda=\frac{b}{a}, x_{i}, z_{i} \in \mathbf{F}_{q}\right\}$. 


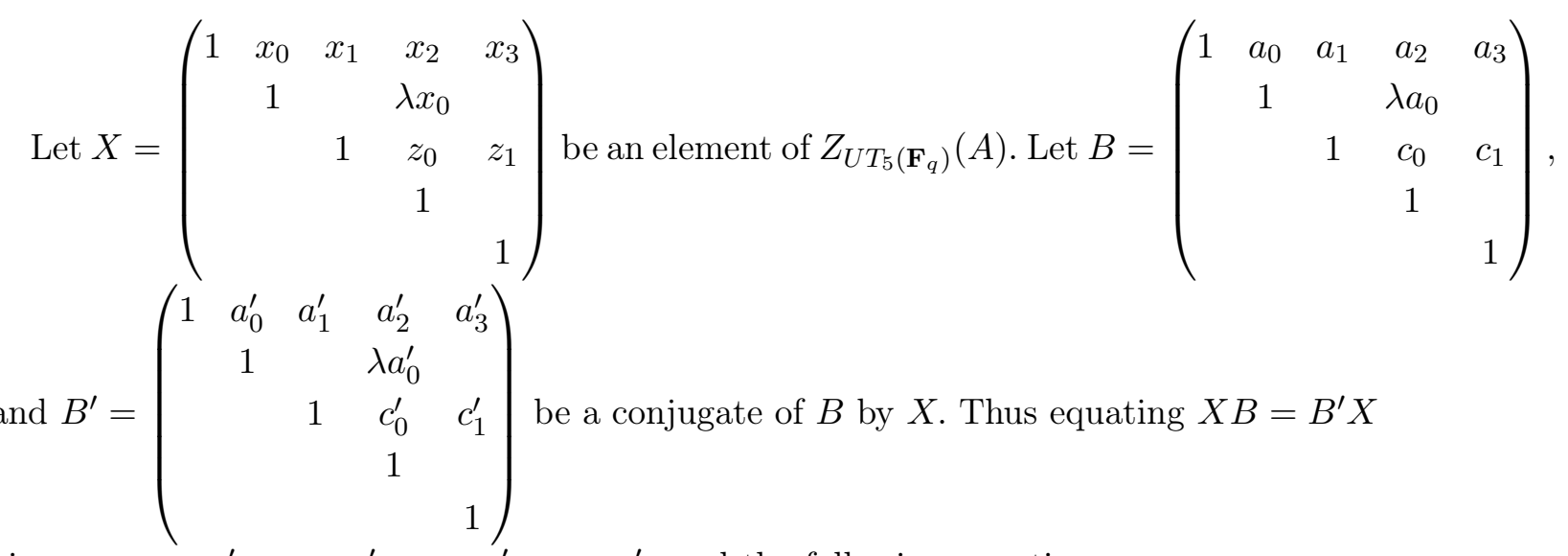

gives us $a_{0}=a_{0}^{\prime}, a_{1}=a_{1}^{\prime}, c_{0}=c_{0}^{\prime}, c_{1}=c_{1}^{\prime}$, and the following equations:

$$
\begin{aligned}
& x_{1} c_{1}+a_{3}=z_{1} a_{1}+a_{3}^{\prime} \\
& x_{1} c_{0}+a_{2}=z_{0} a_{1}+a_{2}^{\prime}
\end{aligned}
$$

We look at two cases, when $\left(a_{1}, c_{0}, c_{1}\right)=\mathbf{0}$ and $\left(a_{1}, c_{0}, c_{1}\right) \neq b f 0$.

Case: $\left(a_{1}, c_{0}, c_{1}\right)=\mathbf{0}$ : In this case, we get $a_{2}=a_{2}^{\prime}$ and $a_{3}=a_{3}^{\prime}$. Therefore $Z_{U T_{5}\left(\mathbf{F}_{q}\right)}(A, B)=$ $Z_{U T_{5}\left(\mathbf{F}_{q}\right)}(A)$. So $(A, B)$ is a branch of type $B_{4}$, and there are $q^{3}$ branches.

Case: $\left(a_{1}, c_{0}, c_{1}\right) \neq \mathbf{0}$ : When $a_{1} \neq 0$, then we choose $z_{0}$ and $z_{1}$ in such a way that we get $a_{2}=a_{3}=0$. By routine check, we get that $Z_{U T_{5}\left(\mathbf{F}_{q}\right)}(A, B)$ is commutative group of size $q^{4}$. Thus $(A, B)$ is of the regular type $R_{3}$, and there are $q^{3}(q-1)$ branches of this type.

When $a_{1}=0$ and one of $c_{0}$ and $c_{1}$ is non-zero. We can choose $x_{1}$ in such a way that we get either $a_{2}=0$ or $a_{3}=0$. Again by simple calculations, we get $Z_{U T_{5}\left(\mathbf{F}_{q}\right)}(A, B)$ is commutative group of size $q^{5}$. Thus $(A, B)$ is of the regular type $R_{2}$, and there are $q^{2}\left(q^{2}-1\right)$ branches of this type.

Proposition 7.11. An upper unitriangular matrix of type $B_{5}$ has $q^{2}$ branches of type $B_{5},\left(q^{5}-q\right)$ branches of regular type $B_{6}$.

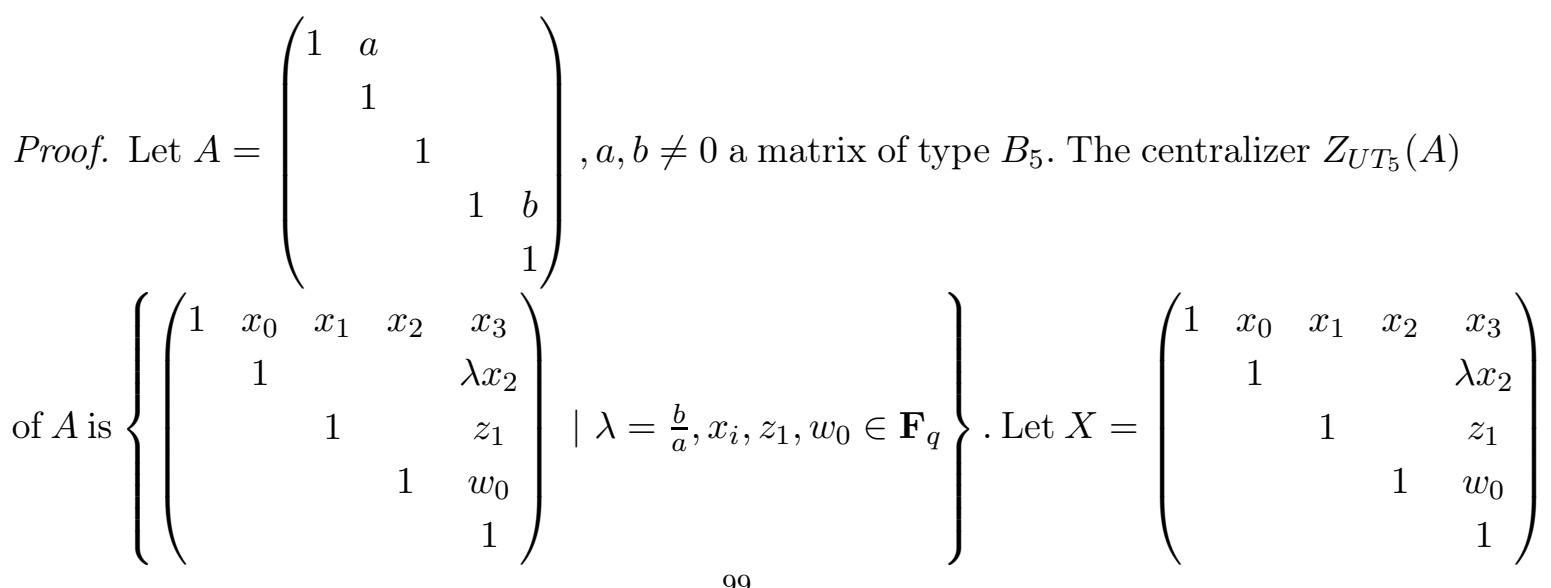




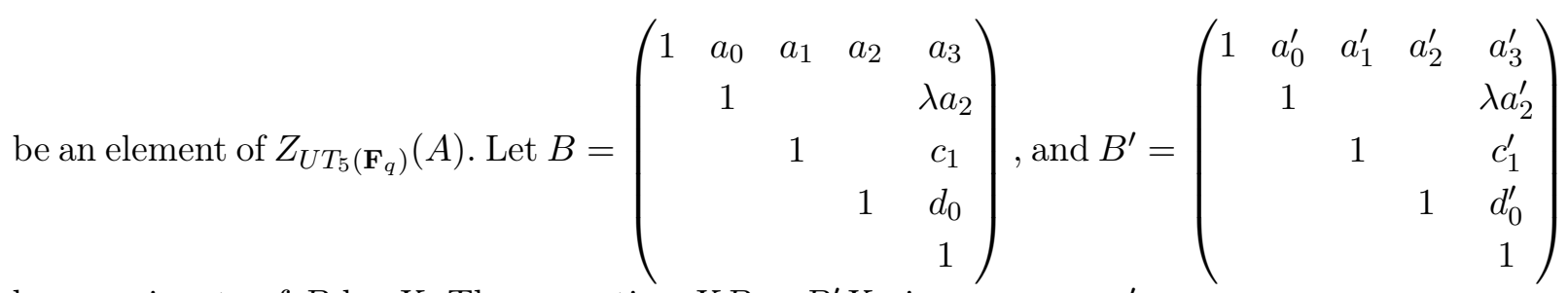

be a conjugate of $B$ by $X$. Thus equating $X B=B^{\prime} X$ gives us $a_{0}=a_{0}^{\prime}$

$a_{1}=a_{1}^{\prime}, a_{2}=a_{2}^{\prime}, c_{1}=c_{1}^{\prime}, d_{0}=d_{0}^{\prime}$, and the following equation:

$$
x_{2} d_{0}+c_{1} x_{1}+\lambda a_{2} x_{0}+a_{3}=\lambda x_{2} a_{0}^{\prime}+z_{1} a_{1}^{\prime}+w_{0} a_{2}^{\prime}+a_{3}^{\prime}
$$

We look at three cases, the first case is when $\lambda a_{0}=d_{0}$ and $\left(a_{1}, a_{2}, c_{1}\right)=\mathbf{0}$. The second case is when $\lambda a_{0} \neq d_{0}$ and the third case is when $\lambda a_{0}=d_{0}$ but $\left(a_{1}, a_{2}, c_{1}\right) \neq \mathbf{0}$.

Case: $\lambda a_{0}=d_{0}$ and $\left(a_{1}, a_{2}, c_{1}\right)=\mathbf{0}$. In this case, we get $a_{3}=a_{3}^{\prime}$. Therefore $Z_{U T_{5}\left(\mathbf{F}_{q}\right)}(A, B)=Z_{U T_{5}\left(\mathbf{F}_{q}\right)}(A)$. So $(A, B)$ is a branch of type $B_{5}$, and there are $q^{2}$ branches.

Case: $\lambda a_{0} \neq d_{0}$ In this case, we can choose $x_{2}$ in such a way that we get $a_{3}=0$. By routine check, we get $Z_{U T_{5}\left(\mathbf{F}_{q}\right)}(A, B)$ is group of size $q^{5}$ isomorphic to centralizer of one of the type $B_{6}$. Thus $(A, B)$ is of type $B_{6}$, and there are $q^{4}(q-1)$ branches of this type.

Case: $\lambda a_{0}=d_{0}$ and $\left(a_{1}, a_{2}, c_{1}\right) \neq \mathbf{0}$. In this case, one of $a_{1}, a_{2}$ and $c_{1}$ is non-zero and depending on this, we can choose one of $z_{1}, w_{0}$ or $x_{1}$ suitably in such a way that we get $a_{3}=0$. By routine check, we get $Z_{U T_{5}\left(\mathbf{F}_{q}\right)}(A, B)$ is group of size $q^{5}$ isomorphic to centralizer of one of the type $B_{6}$. Thus $(A, B)$ is of type $B_{6}$, and there are $q(q-1)\left(q^{2}+q+1\right)$ branches of this type.

Therefore a matrix of type $B_{5}$ has $q^{2}$ branches of type $B_{5}$ and total $q\left(q^{4}-1\right)$ braches of type $B_{6}$.

Proposition 7.12. An upper unitriangular matrix of type $B_{6}$ has $q^{3}$ branches of type $B_{6}$, and $q^{2}\left(q^{2}-1\right)$ branches of regular type $R_{3}$.

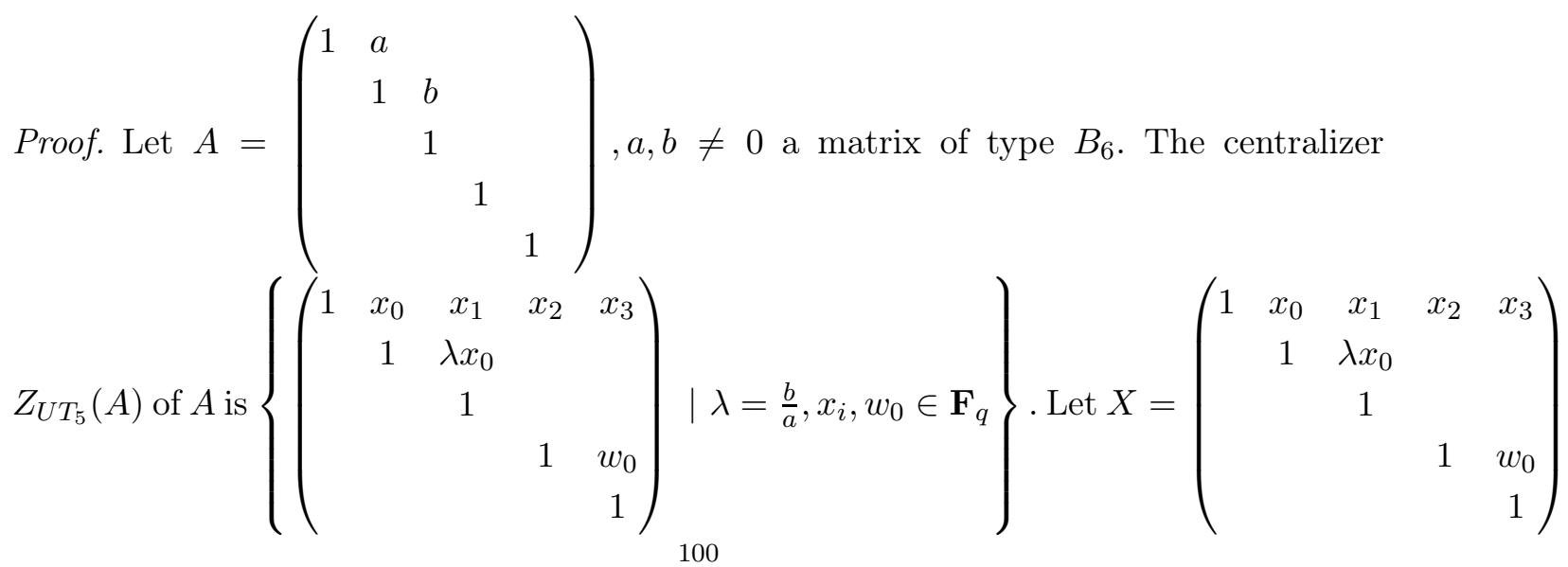


be an element of $Z_{U T_{5}\left(\mathbf{F}_{q}\right)}(A)$. Let $B=\left(\begin{array}{ccccc}1 & a_{0} & a_{1} & a_{2} & a_{3} \\ & 1 & \lambda a_{0} & & \\ & & 1 & & \\ & & & 1 & d_{0} \\ & & & & 1\end{array}\right)$, and $B^{\prime}=\left(\begin{array}{ccccc}1 & a_{0}^{\prime} & a_{1}^{\prime} & a_{2}^{\prime} & a_{3}^{\prime} \\ & 1 & \lambda a_{0}^{\prime} & & \\ & 1 & & \\ & & & 1 & d_{0}^{\prime} \\ & & & & 1\end{array}\right)$

be a conjugate of $B$ by $X$. Thus equating $X B=B^{\prime} X$ gives us $a_{0}=a_{0}^{\prime}, a_{1}=a_{1}^{\prime}, a_{2}=a_{2}^{\prime}$, $d_{0}=d_{0}^{\prime}$, and the following equation:

$$
x_{2} d_{0}+a_{3}=w_{0} a_{2}^{\prime}+a_{3}^{\prime}
$$

We look at two cases, when $\left(a_{2}, d_{0}\right)=(0,0)$ and $\left(a_{2}, d_{0}\right) \neq(0,0)$.

Case: $\left(a_{2}, d_{0}\right)=(0,0)$ In this case, we get $a_{3}=a_{3}^{\prime}$. Therefore $Z_{U T_{5}\left(\mathbf{F}_{q}\right)}(A, B)=$ $Z_{U T_{5}\left(\mathbf{F}_{q}\right)}(A)$. So $(A, B)$ is a branch of type $B_{6}$, and there are $q$ branches.

Case: $\left(a_{2}, d_{0}\right) \neq(0,0)$ In this case, one of $d_{0}$ and $a_{2}$ is non-zero. We can choose $x_{2}$ or $w_{0}$ in such a way that we get $a_{3}=0$. By routine check, we get $Z_{U T_{5}\left(\mathbf{F}_{q}\right)}(A, B)$ is commutative group of size $q^{4}$. Thus $(A, B)$ is of the regular type $R_{3}$, and there are $q^{2}\left(q^{2}-1\right)$ branches of this type.

7.3. Branching of type $D$. Now we look at the branching for type $D$.

Proposition 7.13. An upper unitriangular matrix of type $D_{1}$ has the following branches:

\begin{tabular}{c|c||c|c}
\hline Branch & No. of Branches & Branch & No. of Branches \\
\hline$D_{1}$ & $q^{2}$ & $R_{2}$ & $q^{2}(q-1)$ \\
$B_{4}$ & $2 q(q-1)$ & $R_{3}$ & $q^{2}\left(q^{2}-1\right)$. \\
$U N T_{3}$ & $q(q-1)^{2}$ & & \\
\hline
\end{tabular}

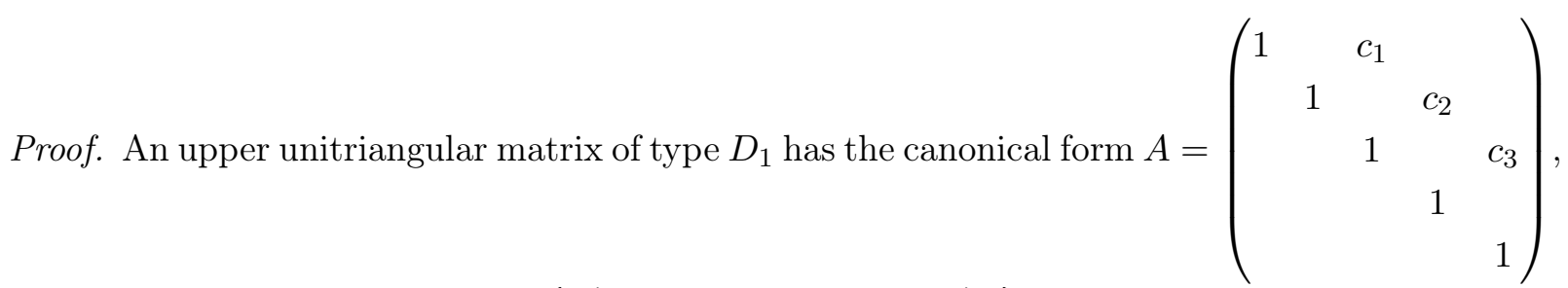
where $a, b, c \neq 0 . Z_{U T_{5}\left(\mathbf{F}_{q}\right)}(A)=\left\{\left(\begin{array}{ccccc}1 & a_{1} & a_{2} & b_{1} & d_{1} \\ & 1 & a_{3} & b_{2} & d_{2} \\ & & 1 & \frac{c_{2}}{c_{1}} a_{1} & \frac{c_{3}}{c_{1}} a_{2} \\ & & & 1 & \frac{c_{3}}{c_{2}} a_{3} \\ & & & & 1\end{array}\right)\right\}$, which we rewrite as: $Z_{U T_{5}\left(\mathbf{F}_{q}\right)}(A)=\left\{\left(\begin{array}{ccc}C & \vec{b} & \vec{d} \\ & 1 & \frac{c_{3}}{c_{2}} C_{23} \\ & & 1\end{array}\right) \mid C \in U T_{3}\left(\mathbf{F}_{q}\right), \vec{b}=\left(\begin{array}{c}b_{1} \\ b_{2} \\ \frac{c_{2}}{c_{1}} C_{12}\end{array}\right), \vec{d}=\left(\begin{array}{c}d_{1} \\ d_{2} \\ c_{3} C_{13}\end{array}\right)\right\}$. 
Let $B=\left(\begin{array}{ccc}C & \vec{b} & \vec{d} \\ & 1 & \frac{c_{3}}{c_{2}} C_{23} \\ & & 1\end{array}\right)$, and $B^{\prime}=\left(\begin{array}{ccc}C^{\prime} & \overrightarrow{b^{\prime}} & \overrightarrow{d^{\prime}} \\ & 1 & \frac{c_{3}}{c_{2}} C_{23}^{\prime} \\ & & 1\end{array}\right)$ be a conjugate of $B$ by a member $X=\left(\begin{array}{ccc}Z & \vec{y} & \vec{w} \\ & 1 & \frac{c_{3}}{c_{2}} Z_{23} \\ & 1\end{array}\right) \in Z_{U T_{5}\left(\mathbf{F}_{q}\right)}(A)$, with $\vec{y}=\left(\begin{array}{c}y_{1} \\ y_{2} \\ \frac{c_{2}}{c_{1}} Z_{12}\end{array}\right)$, and $\vec{w}=\left(\begin{array}{c}w_{1} \\ w_{2} \\ \frac{c_{3}}{c_{1}} Z_{13}\end{array}\right)$.

We thus have $X B=B^{\prime} X$. First thing we see is that $Z C=C^{\prime} Z$. So we can take $C$ to be a conjugacy class representative in $U T_{3}\left(\mathbf{F}_{q}\right)$, and we thus have the following equations:

$$
\begin{aligned}
Z \vec{b}+\vec{y} & =C \vec{y}+\overrightarrow{b^{\prime}} \\
Z \vec{d}+\frac{c_{3}}{c_{2}} C_{23} \vec{y}+\vec{w} & =C^{\prime} \vec{w}+\frac{c_{3}}{c_{2}} Z_{23} \overrightarrow{b^{\prime}}+\overrightarrow{d^{\prime}}
\end{aligned}
$$

When $C=I_{3}$ : In this case $C_{12}=C_{13}=C_{23}=0$. We have $Z=\left(\begin{array}{ccc}1 & z_{1} & z_{2} \\ & 1 & z_{3} \\ & & 1\end{array}\right)$. Equation 7.15 becomes: $\left(\begin{array}{c}b_{1}+z_{1} b_{2} \\ b_{2} \\ 0\end{array}\right)=\left(\begin{array}{c}b_{1}^{\prime} \\ b_{2}^{\prime} \\ 0\end{array}\right)$. We look at two cases here: When $b_{2} \neq 0$, and when $b_{2}=0$.

When $b_{2}=0$, We have $b_{1}^{\prime}=b_{1}$, and Equation [7.16 becomes:

$$
\left(\begin{array}{c}
d_{1}+z_{1} d_{2} \\
d_{2} \\
0
\end{array}\right)=\left(\begin{array}{c}
d_{1}^{\prime}+\frac{c_{3}}{c_{2}} z_{3} b_{1} \\
d_{2}^{\prime}+\frac{c_{3} z_{3}}{c_{2}} b_{2}^{\prime} \\
0
\end{array}\right)
$$

We have $d_{2}^{\prime}=d_{2}$.

When $b_{2}=b_{1}=d_{2}=0$ : We have $d_{1}^{\prime}=d_{1}$. Thus $B$ is reduced to $\left(\begin{array}{ccccc}1 & & & & d_{1} \\ & 1 & & & \\ & 1 & & \\ & & 1 & \\ & & & & 1\end{array}\right)$.

So $Z_{U T_{5}\left(\mathbf{F}_{q}\right)}(A, B)=Z_{U T_{5}\left(\mathbf{F}_{q}\right)}(A)$. Hence $(A, B)$ is a branch of type $D_{1}$, and there are $q$ such branches.

When $b_{2}=b_{1}=0$, and $d_{2} \neq 0$, we can choose $z_{1}$ such that $d_{1}=0$. Thus, $B$ is reduced

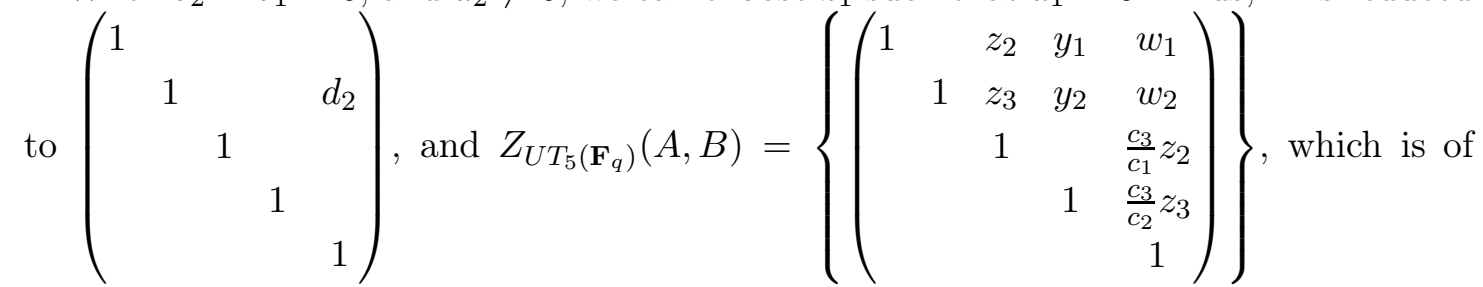
type. So $(A, B)$ is a branch of type $B_{4}$, as $Z_{U T_{5}\left(\mathbf{F}_{q}\right)}(A, B)$ can be conjugated by the 
elementary matrix that swaps rows and columns 1 and 2 to get the centralizer subgroup of one of the canonical matrices of type $B_{4}$, and there are $(q-1)$ branches of this type.

When $b_{1} \neq 0$, in Equation 7.16, we choose $z_{3}$ so that $d_{1}=0$. Thus $B$ is reduced to

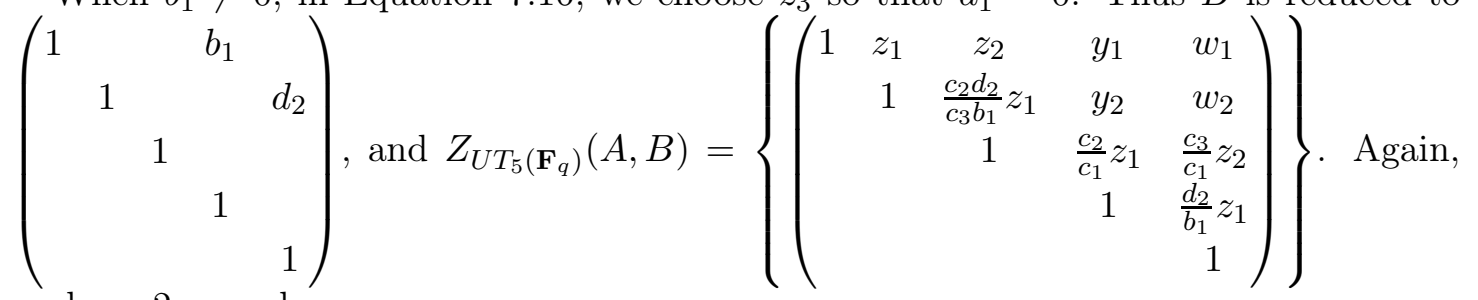

we have 2 cases here:

When $d_{2}=0, B=\left(\begin{array}{ccccc}1 & & & b_{1} & \\ & 1 & & & \\ & & 1 & & \\ & & & 1 & \\ & & & & 1\end{array}\right)$. Here $Z_{U T_{5}\left(\mathbf{F}_{q}\right)}(A, B)=\left\{\left(\begin{array}{ccccc}1 & z_{1} & z_{2} & y_{1} & w_{1} \\ & 1 & & y_{2} & w_{2} \\ & & 1 & \frac{c_{2}}{c_{1}} z_{1} & \frac{c_{3}}{c_{1}} z_{2} \\ & & & 1 & \\ & & & & 1\end{array}\right)\right\}$.

On conjugating by an elementary matrix, which swaps rows and columns 2 and 3 of each element of $Z_{U T_{5}\left(\mathbf{F}_{q}\right)}(A, B)$, we get the centralizer of one of the canonical matrices of the type $B_{4}$. Thus there are $q-1$ branches of type $B_{4}$.

When $d_{2} \neq 0$, we have $Z_{U T_{5}\left(\mathbf{F}_{q}\right)}(A, B)=\left\{\left(\begin{array}{ccccc}1 & z_{1} & z_{2} & y_{1} & w_{1} \\ & 1 & \frac{c_{2} d_{2}}{c_{3} b_{1}} z_{1} & y_{2} & w_{2} \\ & 1 & \frac{c_{2}}{c_{1}} z_{1} & \frac{c_{3}}{c_{1}} z_{2} \\ & & 1 & \frac{d_{2}}{b_{1}} z_{1} \\ & & & \\ & & & & \end{array}\right)\right\}$. Thus this branch is of the new type $U N T_{3}$, and there are $(q-1)^{2}$ such branches.

When $b_{2} \neq 0$, choose $z_{1}$ such that $b_{1}^{\prime}=0$. Thus equating Equation 7.15 with $b_{1}$ replaced by 0 , we get that $z_{1}=0$. Thus with $b_{1}=0$ and $z_{1}=0$, we get from Equation 7.16, $d_{1}^{\prime}=d_{1}$, and with a nice choice of $z_{3}$, we can reduce $d_{2}^{\prime}$ to 0 . Hence, $B$ is reduced to $\left(\begin{array}{ccccc}1 & & & d_{1} \\ & 1 & & b_{2} & \\ & 1 & & \\ & & 1 & \\ & & & 1\end{array}\right)$, and $Z_{U T_{5}\left(\mathbf{F}_{q}\right)}=\left\{\left(\begin{array}{ccccc}1 & & z_{2} & y_{1} & w_{1} \\ & 1 & & y_{2} & w_{2} \\ & 1 & & \frac{c_{3}}{c_{1}} z_{2} \\ & & 1 & \\ & & & & 1\end{array}\right)\right\}$, which is a centralizer of type $R_{2}$. Thus $(A, B)$ is a branch of type $R_{2}$, and there are $q(q-1)$ such branches.

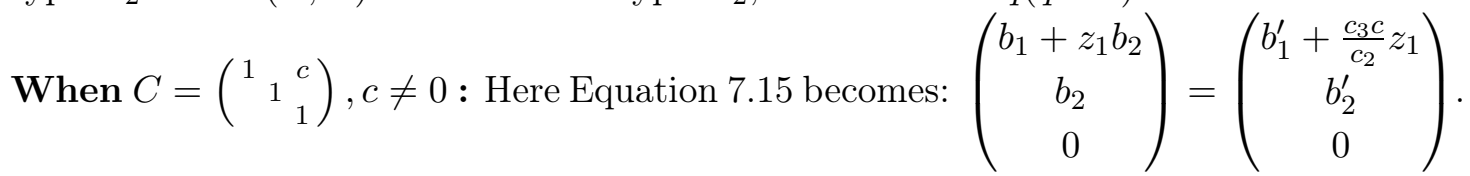
So we have $b_{2}^{\prime}=b_{2}$. We see 2 cases here: $b_{2}=\frac{c_{2}}{c_{1}} c$, and $b_{2} \neq \frac{c_{2}}{c_{1}} c$.

When $b_{2} \neq \frac{c_{2}}{c_{1}} c$. In the above equation, we choose $z_{1}$ such that $b_{1}^{\prime}=0$. Thus, with substituting $b_{1}$ with $b_{1}^{\prime}=0$ in the above equation, we get $z_{1}=0$. Thus, with this, 
Equation 7.16 becomes $\left(\begin{array}{c}d_{1} \\ d_{2}+\frac{c_{3}}{c_{1}} c z_{3} \\ \frac{c_{3}}{c_{1}} c\end{array}\right)=\left(\begin{array}{c}d_{1}^{\prime} \\ d_{2}^{\prime}+\frac{c_{3}}{c_{2}} b_{2} z_{3} \\ \frac{c_{3}}{c_{1}} c\end{array}\right)$. As $b_{2} \neq \frac{c_{2}}{c_{1}} c$, we can choose a $z_{3}$ so that $d_{2}^{\prime}=0$, and we have $d_{1}^{\prime}=d_{1}$. So $B$ boils down to $\left(\begin{array}{ccccc}1 & & c & & d_{1} \\ & 1 & & b_{2} & \\ & 1 & & \frac{c_{3}}{c_{1}} c \\ & & 1 & \\ & & & & 1\end{array}\right)$, with $Z_{U T_{5}\left(\mathbf{F}_{q}\right)}(A, B)=\left\{\left(\begin{array}{ccccc}1 & & z_{2} & y_{1} & w_{1} \\ & 1 & & y_{2} & w_{2} \\ & & 1 & & \frac{c_{3}}{c_{1}} z_{2} \\ & & & 1 & \\ & & & & 1\end{array}\right)\right\}$. Thus $(A, B)$ too is a branch of type $R_{2}$, and there are $q(q-1)^{2}$ such branches.

When $b_{2}=\frac{c_{2}}{c_{1}} c$, we get from Equation [7.15, $b_{1}^{\prime}=b_{1}$. Equation 7.16 boils down to: $\left(\begin{array}{c}d_{1}+z_{1} d_{2} \\ d_{2} \\ 0\end{array}\right)=\left(\begin{array}{c}d_{1}^{\prime}+\frac{c_{3}}{c_{2}} z_{3} b_{1} \\ d_{2}^{\prime} \\ 0\end{array}\right)$. So we have $d_{2}^{\prime}=d_{2}$. We look first at $b_{1}=d_{2}=0 . B$ is reduced to $\left(\begin{array}{ccccc}1 & & c & & d_{1} \\ & 1 & & \frac{c_{2}}{c_{1}} c & \\ & & 1 & & \frac{c_{3}}{c_{1}} c \\ & & 1 & \\ & & & 1\end{array}\right)$, and $Z_{U T_{5}\left(\mathbf{F}_{q}\right)}(A, B)=Z_{U T_{5}\left(\mathbf{F}_{q}\right)}(A)$. Thus $(A, B)$ is a branch of type $D_{1}$, and there are $q(q-1)$ such branches.

When $b_{1} \neq 0$ choose $z_{3}$ such that $d_{1}^{\prime}=0$. So, $B$ becomes: $\left(\begin{array}{cccccc}1 & & c & b_{1} & \\ & 1 & & \frac{c_{2}}{c_{1}} c & d_{2} \\ & & 1 & & \frac{c_{3}}{c_{1}} c \\ & & 1 & \\ & & & & 1\end{array}\right)$. We have two cases here: 


$$
\begin{aligned}
\text { When } d_{2}=0 \text {, we have } B=\left(\begin{array}{ccccc}
1 & & c & b_{1} & \\
& 1 & & \frac{c_{2}}{c_{1}} c & \\
& 1 & & \frac{c_{3}}{c_{1}} c \\
& & & \\
& & & 1
\end{array}\right) \text { and } \\
\qquad Z_{U T_{5}\left(\mathbf{F}_{q}\right)}(A, B)=\left\{\left(\begin{array}{ccccc}
1 & z_{1} & z_{2} & y_{1} & w_{1} \\
& 1 & & y_{2} & w_{2} \\
& & 1 & \frac{c_{2}}{c_{1}} z_{1} & \frac{c_{3}}{c_{1}} z_{2} \\
& & & 1 & \\
& & & & 1
\end{array}\right)\right\},
\end{aligned}
$$

thus $(A, B)$ is of a type $B_{4}$, and there are $(q-1)^{2}$ such branches.

$$
\begin{aligned}
& \text { When } d_{2} \neq 0, B=\left(\begin{array}{ccccc}
1 & & c & b_{1} & \\
& 1 & & \frac{c_{2}}{c_{1}} c & d_{2} \\
& 1 & & \frac{c_{3}}{c_{1}} c \\
& & 1 & \\
& & & 1
\end{array}\right) \text {, and } \\
& Z_{U T_{5}\left(\mathbf{F}_{q}\right)}(A, B)=\left\{\left(\begin{array}{ccccc}
1 & z_{1} & z_{2} & y_{1} & w_{1} \\
& 1 & \frac{c_{2} d_{2}}{c_{3} b_{1}} z_{1} & y_{2} & w_{2} \\
& & 1 & \frac{c_{2}}{c_{1}} z_{1} & \frac{c_{3}}{c_{1}} z_{2} \\
& & & 1 & \frac{d_{2}}{b_{1}} z_{1} \\
& & & & 1
\end{array}\right)\right\},
\end{aligned}
$$

so, this branch too is of the type $U N T_{3}$. Thus there are $(q-1)^{3}$ branches of this new type.

When $b_{1}=0$, and $d_{2} \neq 0$. We choose $z_{1}$ so that $d_{1}^{\prime}=0$. Thus $B$ is reduced to

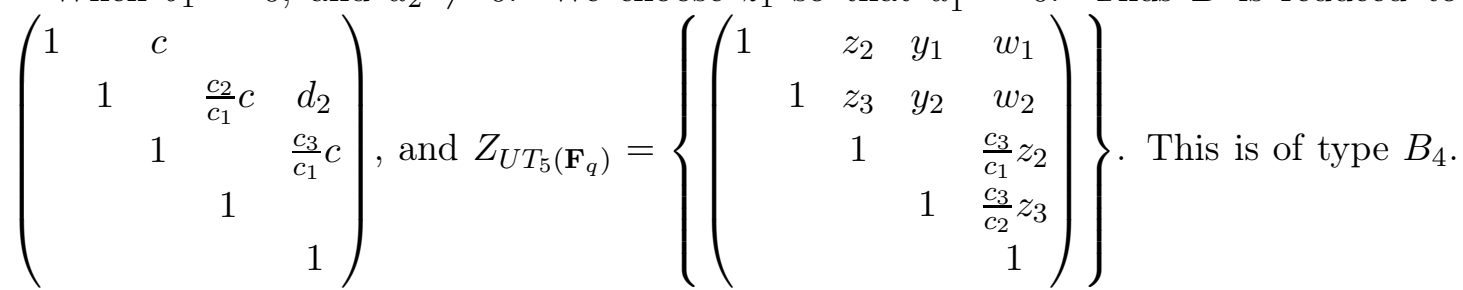
$(A, B)$ is a branch of type $B_{4}$, and there are $(q-1)^{2}$ such branches.

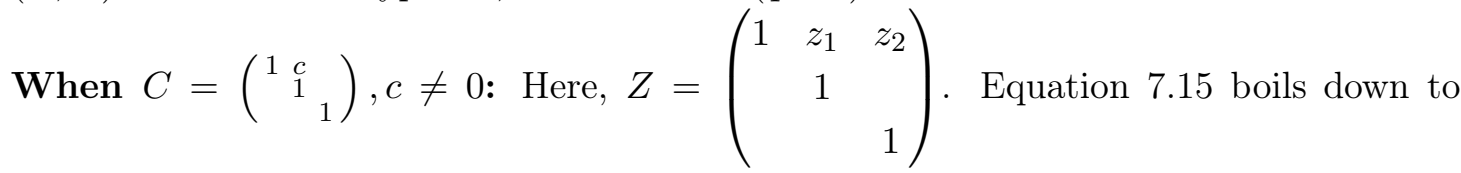
$\left(\begin{array}{c}b_{1}+b_{2} z_{1}+\frac{c_{2}}{c_{1}} c z_{2} \\ b_{2} \\ \frac{c_{2}}{c_{1}} c\end{array}\right)=\left(\begin{array}{c}c y_{2}+b_{1}^{\prime} \\ b_{2}^{\prime} \\ \frac{c_{2}}{c_{1}} c\end{array}\right)$. So $b_{2}^{\prime}=b_{2}$. As $c \neq 0$, we choose $y_{2}$ such that 
$b_{1}^{\prime}=0$. Equation 7.16 becomes: $\left(\begin{array}{c}d_{1}+d_{2} z_{1} \\ d_{2} \\ 0\end{array}\right)=\left(\begin{array}{c}c w_{2}+d_{1}^{\prime} \\ d_{2}^{\prime} \\ 0\end{array}\right)$. We have $d_{2}^{\prime}=d_{2}$. Take $w_{2}$ such that $d_{1}^{\prime}=0$. So $B$ is reduced to $\left(\begin{array}{ccccc}1 & c & & & \\ & 1 & & b_{2} & d_{2} \\ & 1 & \frac{c_{2}}{c_{1}} c & \\ & & 1 & \\ & & & 1\end{array}\right)$, and therefore $Z_{U T_{5}\left(\mathbf{F}_{q}\right)}(A, B)=\left\{\left(\begin{array}{ccccc}1 & z_{1} & z_{2} & y_{1} & y_{2} \\ & 1 & & \frac{b_{2}}{c} z_{1}+\frac{c_{2}}{c_{1}} z_{2} & \frac{d_{2}}{c} z_{1} \\ & 1 & \frac{c_{2}}{c_{1}} z_{1} & \frac{c_{3}}{c_{1}} z_{2} \\ & & & 1 & \\ & & & & 1\end{array}\right)\right\}$, which is of size $q^{4}$. It is routine to check that this centralizer is commutative. Thus this is a centralzier of type $R_{3}$. Thus $(A, B)$ is a branch of type $R_{3}$, and there are $q^{2}(q-1)$ such branches.

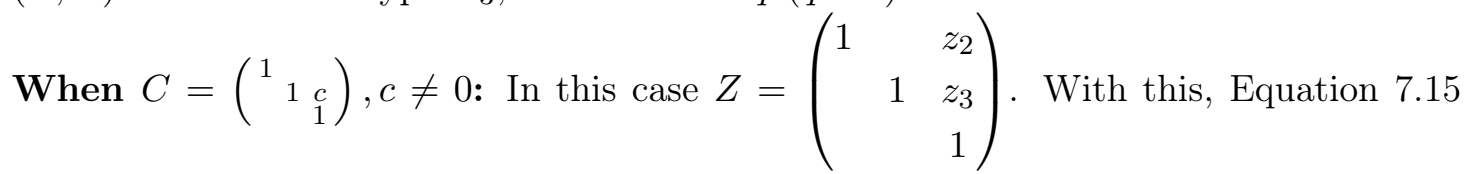
becomes $\left(\begin{array}{c}b_{1} \\ b_{2} \\ 0\end{array}\right)=\left(\begin{array}{c}b_{1}^{\prime} \\ b_{2}^{\prime} \\ 0\end{array}\right)$. So, our focus thus is solely on Equation 7.16. The equation is reduced to $\left(\begin{array}{c}d_{1}+\frac{c_{3}}{c_{2}} c y_{1} \\ d_{2}+\frac{c_{3}}{c_{2}} c y_{2} \\ 0\end{array}\right)=\left(\begin{array}{c}d_{1}^{\prime}+\frac{c_{3}}{c_{2}} b_{1} z_{3} \\ d_{2}^{\prime}+\frac{c_{3}}{c_{1}} c z_{2}+\frac{c_{3}}{c_{2}} b_{2} z_{3} \\ 0\end{array}\right)$

As $\frac{c_{3}}{c_{2}} c \neq 0$, choose $y_{1}, y_{2}$ so that $d_{1}^{\prime}=d_{2}^{\prime}=0$. Thus $B$ is reduced to $\left(\begin{array}{cccccc}1 & & & b_{1} & \\ & 1 & c & b_{2} & \\ & 1 & & \\ & & 1 & \frac{c_{3}}{c_{2}} c \\ & & & & 1\end{array}\right)$, and $Z_{U T_{5}\left(\mathbf{F}_{q}\right)}(A, B)=\left\{\left(\begin{array}{ccccc}1 & & z_{2} & \frac{b_{1}}{c} z_{3} & w_{1} \\ & 1 & z_{3} & \frac{c_{2}}{c_{1}} z_{2}+\frac{b_{2}}{c} z_{3} & w_{2} \\ & 1 & & \frac{c_{3}}{c_{1}} z_{2} \\ & & 1 & \frac{c_{3}}{c_{2}} z_{3} \\ & & & & 1\end{array}\right)\right\}$. This is of size $q^{4}$, and with a routine check we see that it is commutative. This is a centralizer of type $R_{3}$, hence $(A, B)$ is a branch of type $R_{3}$, and there are $q^{2}(q-1)$ such branches. 
When $C=\left(\begin{array}{ccc}1 & c_{0} & \\ & 1 & d_{0}\end{array}\right), c_{0}, d_{0} \neq 0$ : Here $Z=\left(\begin{array}{ccc}1 & z_{1} & z_{2} \\ & & 1\end{array}\right)$, where $\lambda_{0}=\frac{d_{0}}{c_{0}}$. Equation 7.15 becomes: $\left(\begin{array}{c}b_{1}+z_{1} b_{2}+z_{2} \frac{c_{2}}{c_{1}} c_{0} \\ b_{2}+\frac{c_{2}}{c_{1}} d_{0} z_{1} \\ 0\end{array}\right)=\left(\begin{array}{c}c_{0} y_{2}+b_{1}^{\prime} \\ \frac{c_{2}}{c_{1}} d_{0} z_{1}+b_{2}^{\prime} \\ 0\end{array}\right)$. As $c_{0}$ and $d_{0}$ are nonzero, we have $b_{2}^{\prime}=b_{2}$. We choose $y_{2}$ such that $b_{1}^{\prime}=0$. Hence, on replacing $b_{1}$ with 0 in the above equation we get $y_{2}=\frac{b_{2}}{c_{0}} z_{1}+\frac{c_{2}}{c_{1}} z_{2}$. With these, Equation 7.16 boils down to $\left(\begin{array}{c}d_{1}+z_{1} d_{2}+\frac{c_{3}}{c_{2}} d_{0} y_{1} \\ d_{2} \\ 0\end{array}\right)=\left(\begin{array}{c}c_{0} w_{2}+d_{1}^{\prime} \\ d_{2}^{\prime} \\ 0\end{array}\right)$. So $d_{2}^{\prime}=d_{2}$, and choose $w_{2}$ such that $d_{1}^{\prime}=0$. Hence, $B$ is reduced to $\left(\begin{array}{ccccc}1 & c_{0} & & & \\ & 1 & d_{0} & b_{2} & d_{2} \\ & & 1 & \frac{c_{2}}{c_{1}} c_{0} & \\ & & & 1 & \frac{c_{3}}{c_{2}} d_{0} \\ & & & & 1\end{array}\right)$, with $Z_{U T_{5}\left(\mathbf{F}_{q}\right)}(A, B)=$ $\left\{\left(\begin{array}{ccccc}1 & z_{1} & z_{2} & y_{1} & w_{1} \\ & 1 & \frac{d_{0}}{c_{0}} z_{1} & \frac{b_{2}}{c_{0}} z_{1}+\frac{c_{2}}{c_{1}} z_{2} & \frac{c_{3}}{c_{2} c_{0}} y_{1}+\frac{d_{2} c_{0}}{d_{0}} z_{1} \\ & 1 & \frac{c_{2}}{c_{1}} z_{1} & \frac{c_{3}}{c_{1}} z_{2} \\ & & 1 & \frac{c_{3} d_{0}}{c_{2} c_{0}} z_{1} \\ & & & 1\end{array}\right\}\right.$. This too is of type $R_{3}$. So $(A, B)$ is a brach of type $R_{3}$, and there are $q^{2}(q-1)^{2}$ such branches.

So, on adding up the branches of each of the types, we have

- $q^{2}$ branches of type $D_{1}$,

- $2 q(q-1)$ branches of type $B_{4}$,

- $q^{2}(q-1)$ branches of type $R_{2}$,

- $q^{2}\left(q^{2}-1\right)$ branches of type $R_{3}$, and

- $q(q-1)^{2}$ branches of type $U N T_{3}$.

These match with the estimations done for $q=3$ in GAP.

Proposition 7.14. An upper unitriangular matrix of type $D_{2}$ has $q^{3}$ branches of type $D_{2}$, and $q^{2}\left(q^{2}-1\right)$ branches of regular type $R_{3}$. 
Proof. Let $A=\left(\begin{array}{ccccc}1 & a & & & \\ & 1 & b & & \\ & & 1 & & c \\ & & & \\ & & & & \\ & & & & 1\end{array}\right), a, b, c \neq 0$ a matrix of type $D_{2}$. The centralizer

$Z_{U T_{5}}(A)$ of $A$ is $\left\{\left(\begin{array}{ccccc}1 & x_{0} & x_{1} & x_{2} & x_{3} \\ & 1 & \lambda_{1} x_{0} & & \lambda_{2} x_{1} \\ & & 1 & & \lambda_{2} x_{0} \\ & & & 1 & w_{0} \\ & & & & 1\end{array}\right) \mid \lambda_{1}=\frac{b}{a}, \lambda_{2}=\frac{c}{a}, x_{i}, w_{0} \in \mathbf{F}_{q}\right\}$

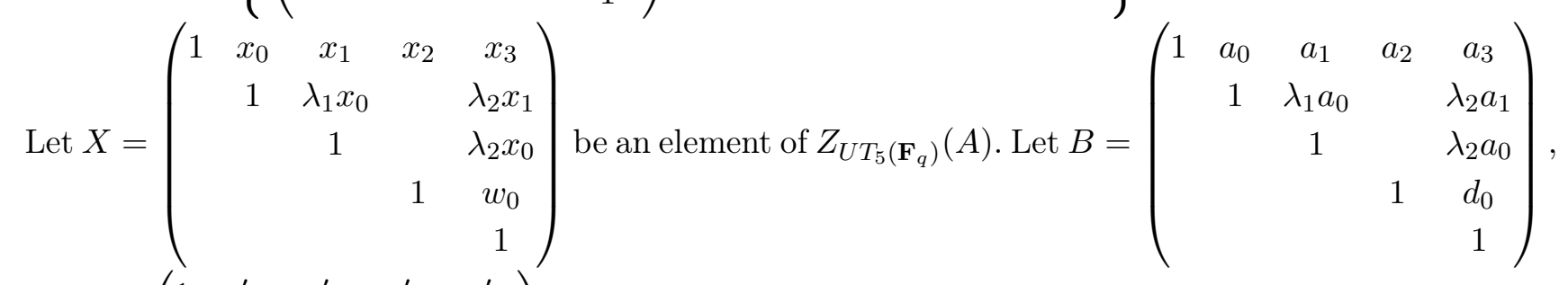

and $B^{\prime}=\left(\begin{array}{ccccc}1 & a_{0}^{\prime} & a_{1}^{\prime} & a_{2}^{\prime} & a_{3}^{\prime} \\ & 1 & \lambda_{1} a_{0}^{\prime} & & \lambda_{2} a_{1}^{\prime} \\ & & 1 & & \lambda_{2} a_{0}^{\prime} \\ & & & 1 & d_{0}^{\prime} \\ & & & & 1\end{array}\right)=X B X^{-1}$. Thus equating $X B=B^{\prime} X$ gives us

$a_{0}=a_{0}^{\prime}, a_{1}=a_{1}^{\prime}, a_{2}=a_{2}^{\prime}, d_{0}=d_{0}^{\prime}$, and the following equation:

$$
x_{2} d_{0}+a_{3}=w_{0} a_{2}^{\prime}+a_{3}^{\prime}
$$

We look at two cases, when $\left(a_{2}, d_{0}\right)=(0,0)$ and $\left(a_{2}, d_{0}\right) \neq(0,0)$.

Case: $\left(a_{2}, d_{0}\right)=(0,0)$ In this case, we get $a_{3}=a_{3}^{\prime}$. Therefore $Z_{U T_{5}\left(\mathbf{F}_{q}\right)}(A, B)=$ $Z_{U T_{5}\left(\mathbf{F}_{q}\right)}(A)$. So $(A, B)$ is a branch of type $D_{2}$, and there are $q$ branches.

Case: $\left(a_{2}, d_{0}\right) \neq(0,0)$ In this case, one of $d_{0}$ and $a_{2}$ is non-zero. We can choose $x_{2}$ or $w_{0}$ in such a way that we get $a_{3}=0$. By routine check, we get $Z_{U T_{5}\left(\mathbf{F}_{q}\right)}(A, B)$ is commutative group of size $q^{4}$. Thus $(A, B)$ is of the regular type $R_{3}$, and there are $q^{2}\left(q^{2}-1\right)$ branches of this type.

Proposition 7.15. A matrix of the $R_{1}$ type has $q^{6}$ branches of type $R_{1}$, a matrix of the $R_{2}$ type has $q^{5}$ branches of type $R_{2}$, and a matrix of the $R_{3}$ type has $q^{4}$ branches of type $R_{3}$.

Proof. The type $R_{1}, R_{2}$ and $R_{3}$ are Regular types, hence the centralizer of matrices of such a type is a commutative.

7.4. Branching Rules for the New Types. While determining the branching rules for the types in $U T_{5}\left(\mathbf{F}_{q}\right)$, we observed that there are some commuting pairs of elements of 
$U T_{5}\left(\mathbf{F}_{q}\right)$, which are not isomorphic to the centralizers of any of the elements in $U T_{5}\left(\mathbf{F}_{q}\right)$. Thus, giving rise to what we call "new types". The new types, we have seen so far are $U N T_{1}$ (first observed in Proposition 7.4), $U N T_{2}$ (observed in Proposition 7.7) and $U N T_{3}$ (observed in Propositions 7.7). Now, we compute the branching for these cases and we see that no further new types occur.

Proposition 7.16. The new type $U N T_{1}$ has $q^{3}$ branches of type $U N T_{1}, q^{2}\left(q^{2}-1\right)$ branches of type $R_{2}$, and $q^{4}-q^{3}$ branches of type $R_{3}$.

Proof. For some pair $(A, B)$ of commuting elements in $U T_{5}\left(\mathbf{F}_{q}\right)$, of type $U N T_{1}$, the centralizer subgroup is $Z_{U T_{5}\left(\mathbf{F}_{q}\right)}(A, B)=\left\{\left(\begin{array}{ccccc}1 & x_{0} & x_{1} & \lambda z_{0} & x_{3} \\ 1 & y_{0} & & y_{2} \\ & 1 & 1 & z_{0}\end{array}\right)\right\}$, where $\lambda \neq 0$ is fixed. Let $C=\left(\begin{array}{ccccc}1 & a_{0} & a_{1} & \lambda c_{0} & a_{3} \\ & 1 & b_{0} & & b_{2} \\ & & 1 & & \\ & & & 1 & c_{0} \\ & & & & 1\end{array}\right)$, and let $C^{\prime}=\left(\begin{array}{ccccc}1 & a_{0}^{\prime} & a_{1}^{\prime} & \lambda c_{0}^{\prime} & a_{3}^{\prime} \\ & 1 & b_{0}^{\prime} & & b_{2}^{\prime} \\ & 1 & & \\ & & 1 & c_{0} \\ & & & & 1\end{array}\right)=X C X^{-1}$, where $X=\left(\begin{array}{ccccc}1 & x_{0} & x_{1} & \lambda z_{0} & x_{3} \\ & 1 & y_{0} & & y_{2} \\ & & 1 & & \\ & & & 1 & z_{0} \\ & & & & 1\end{array}\right)$. On equation $X C=C X$, we get $a_{0}^{\prime}=a_{0}, b_{0}^{\prime}=b_{0}, b_{2}^{\prime}=b_{2}$, $c_{0}^{\prime}=c_{0}$, and the following equations:

$$
\begin{aligned}
& a_{1}+x_{0} b_{0}=a_{1}^{\prime}+a_{0} y_{0} \\
& a_{3}+x_{0} b_{2}=a_{3}^{\prime}+y_{2} a_{0}
\end{aligned}
$$

We look at two main cases: $\left(a_{0}, b_{2}\right)=(0,0)$, and $\left(a_{0}, b_{2}\right) \neq(0,0)$.

When $a_{0}=b_{2}=0$ : Equation 7.18 becomes $a_{3}^{\prime}=a_{3}$, Equation 7.17 becomes $a_{1}^{\prime}=$ $a_{1}+x_{0} b_{0}$. We have two subcases here:

When $b_{0}=0$, then we get $a_{1}^{\prime}=a_{1}$. Thus $C$ boils down to $\left(\begin{array}{ccccc}1 & & a_{1} & \lambda c_{0} & a_{3} \\ & 1 & & & \\ & & 1 & & \\ & & & 1 & c_{0} \\ & & & & 1\end{array}\right)$, and $Z_{U T_{5}\left(\mathbf{F}_{q}\right)}(A, B, C)=Z_{U T_{5}\left(\mathbf{F}_{q}\right)}(A, B) .(A, B, C)$ is therefore of type $U N T_{1}$, and there are $q^{3}$ such branches. 
When $b_{0} \neq 0$, in Equation 7.17, we can choose $x_{0}$ such that $a_{1}^{\prime}=0$. Hence $C$ is reduced

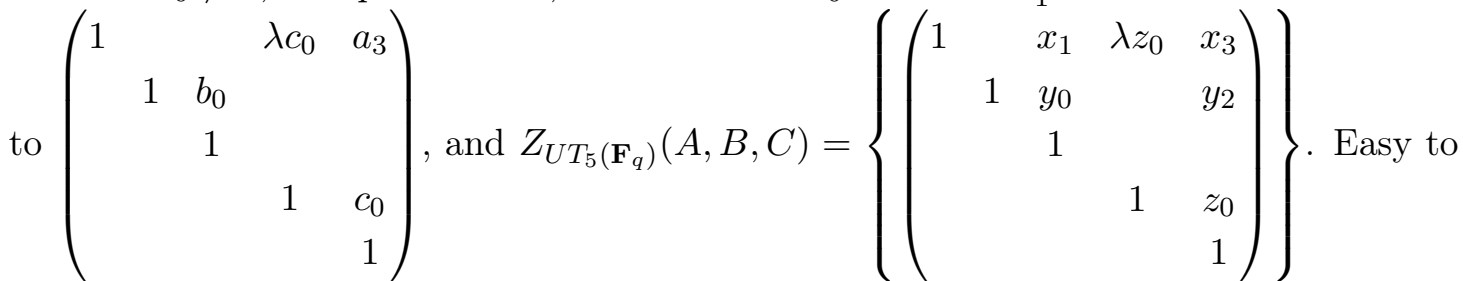
see that this is a commutative group of size $q^{5} .(A, B, C)$ is a branch of type $R_{2}$, and there are $q^{2}(q-1)$ such branches.

When $\left(a_{0}, b_{2}\right) \neq(0,0)$ : When $a_{0} \neq 0$, in Equation 7.17, we choose $y_{0}$ such that $a_{1}^{\prime}=0$. Thus, on replacing $a_{1}$ with $a_{1}^{\prime}=0$ in that equation, we get $y_{0}=\frac{b_{0}}{a_{0}} x_{0}$. In Equation 7.18 choose $y_{2}$ so that $a_{3}^{\prime}=0$. Thus $C$ is reduced to $\left(\begin{array}{cccccc}1 & a_{0} & & \lambda c_{0} & \\ & 1 & b_{0} & & & b_{2} \\ & 1 & & \\ & & 1 & c_{0} \\ & & & & 1\end{array}\right)$, and thus $Z_{U T_{5}\left(\mathbf{F}_{q}\right)}(A, B, C)=\left\{\left(\begin{array}{ccccc}1 & x_{0} & x_{1} & \lambda z_{0} & x_{3} \\ & 1 & \frac{b_{0}}{a_{0}} x_{0} & & \frac{b_{2}}{a_{0}} x_{0} \\ & & 1 & & y_{1} \\ & & & 1 & z_{0} \\ & & & & 1\end{array}\right)\right\}$. Easy to see that this subgroup is a commutative one of size $q^{4}$. Thus $(A, B, C)$ is a branch of type $R_{3}$, and there are $(q-1) q^{3}=q^{4}-q^{3}$ such branches.

When $a_{0}=0$, and $b_{2} \neq 0$. Equation 7.18 becomes $a_{3}+x_{0} b_{2}=a_{3}^{\prime}$, and Choose $x_{0}$ such that $a_{3}^{\prime}=0$. Then, on replacing $a_{3}$ with $a_{3}^{\prime}=0$ in Equation 7.18, we get $x_{0}=0$. With

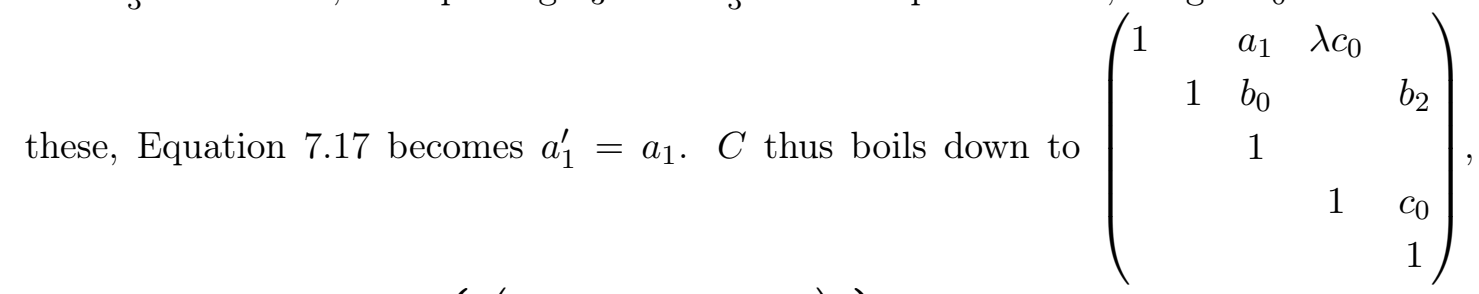
and $Z_{U T_{5}\left(\mathbf{F}_{q}\right)}(A, B, C)=\left\{\left(\begin{array}{ccccc}1 & & x_{1} & \lambda z_{0} & x_{3} \\ & 1 & y_{0} & & y_{2} \\ & 1 & & \\ & & 1 & z_{0} \\ & & & 1\end{array}\right)\right\}$. This branch too is of type $R_{2}$, and there are $q^{3}(q-1)$ such branches. So, in total there are $q^{3}(q-1)+q^{2}(q-1)=q^{4}-q^{2}$ branches of type $R_{2}$.

Proposition 7.17. The new type $U N T_{2}$ has $q^{3}$ branches of type $U N T_{2}, q^{5}-q^{2}$ branches of type $R_{1}$, and $q^{4}-q^{3}$ branches of type $R_{3}$. 
Proof. A commuting pair $(A, B)$ of type $U N T_{2}$ has the centralizer $\left\{\left(\begin{array}{ccccc}1 & x_{1} & y_{1} & y_{2} & x_{2} \\ & 1 & z_{1} & z_{2} & w_{1} \\ & & 1 & & \lambda x_{1} \\ & & & 1 & x_{1} \\ & & & & 1\end{array}\right)\right\}$. Let $C=\left(\begin{array}{ccccc}1 & a_{1} & b_{1} & b_{2} & a_{2} \\ & 1 & c_{1} & c_{2} & d_{1} \\ & & 1 & & \lambda a_{1} \\ & & & 1 & a_{1} \\ & & & & 1\end{array}\right), C^{\prime}=\left(\begin{array}{ccccc}1 & a_{1}^{\prime} & b_{1}^{\prime} & b_{2}^{\prime} & a_{2}^{\prime} \\ & 1 & c_{1}^{\prime} & c_{2}^{\prime} & d_{1} \\ & & 1 & & \lambda a_{1}^{\prime} \\ & & & 1 & a_{1}^{\prime} \\ & & & & 1\end{array}\right)$ be a conjugate of $C$, and let $X=\left(\begin{array}{ccccc}1 & x_{1} & y_{1} & y_{2} & x_{2} \\ & 1 & z_{1} & z_{2} & w_{1} \\ & & 1 & & \lambda x_{1} \\ & & & 1 & x_{1} \\ & & & & 1\end{array}\right)$ such that $X C=C^{\prime} X$. Equating $X C=C^{\prime} X$ gives us $a_{1}^{\prime}=a_{1}, c_{1}^{\prime}=c_{1}$ and $c_{2}^{\prime}=c_{2}$, and the following bunch of equations:

$$
\begin{aligned}
\left(\begin{array}{lll}
a_{1} & b_{1}+x_{1} c_{1} & b_{2}+x_{1} c_{2}
\end{array}\right) & =\left(\begin{array}{lll}
a_{1} & b_{1}^{\prime}+a_{1} z_{1} & b_{2}^{\prime}+a_{1} z_{2}
\end{array}\right) \\
d_{1}+\left(\lambda z_{1}+z_{2}\right) a_{1} & =d_{1}^{\prime}+\left(\lambda c_{1}+c_{2}\right) x_{1} \\
a_{2}+x_{1} d_{1}+\left(\lambda y_{1}+y_{2}\right) a_{1} & =a_{2}^{\prime}+\left(\lambda b_{1}^{\prime}+b_{2}^{\prime}\right) x_{1}+w_{1} a_{1}
\end{aligned}
$$

There are two main cases here:

Case: $a_{1}=0$

When $c_{1}=c_{2}=0$, Equation 7.19 leads us to $b_{1}^{\prime}=b_{1}, b_{2}^{\prime}=b_{2}$, and from Equation 7.20 $d_{1}^{\prime}=d_{1}$. With these, Equation 7.21 becomes $a_{2}+x_{1} d_{1}=a_{2}^{\prime}+\left(\lambda b_{1}+b_{2}\right) x_{1}$.

When $d_{1}=\lambda b_{1}+b_{2}$, we get $a_{2}^{\prime}=a_{2}$. Thus $C$ is reduced to $\left(\begin{array}{ccccc}1 & & b_{1} & b_{2} & a_{2} \\ & 1 & & & \lambda b_{1}+b_{2} \\ & 1 & & \\ & & 1 & \\ & & & \\ & & & 1\end{array}\right)$, and $Z_{U T_{A, B, C}\left(\mathbf{F}_{q}\right)}=Z_{U T_{5}\left(\mathbf{F}_{q}\right)}(A, B)$. Thus, $(A, B, C)$ is of type $U N T_{2}$, and there are $q^{3}$ such branches.

When $d_{1} \neq \lambda b_{1}+b_{2}$, we can choose $x_{1}$ such that $a_{2}^{\prime}=0$. Thus $C$ is reduced to

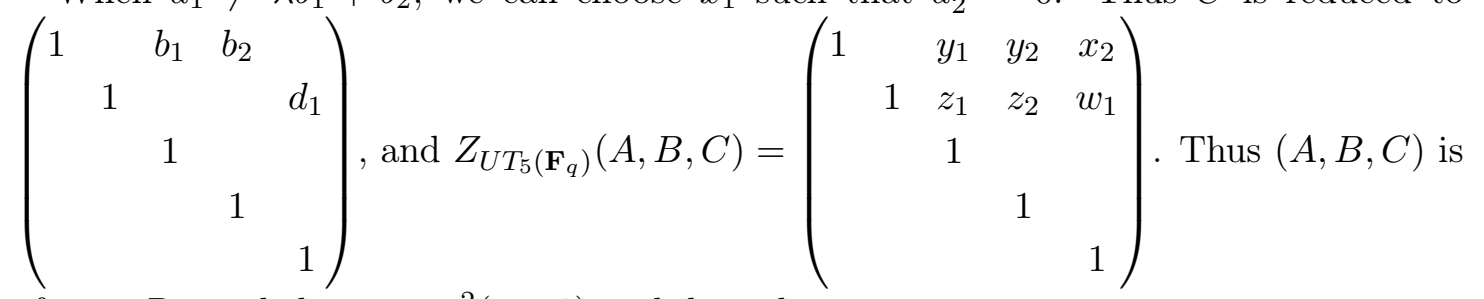
of type $R_{1}$, and there are $q^{2}(q-1)$ such branches. 
When $c_{1} \neq 0$, in Equation [7.19, we can choose $x_{1}$ so that $b_{1}^{\prime}=0$. Thus on replacing $b_{1}$ with $b_{1}^{\prime}=0$, we get $x_{1}=0$, and thus $b_{2}^{\prime}=b_{2}$. And Equation 7.20 reduces to $d_{1}^{\prime}=d_{1}$, and Equation 7.21 boils down to $a_{2}^{\prime}=a_{2}$. C is reduced to $\left(\begin{array}{cccccc}1 & & & b_{2} & a_{2} \\ & 1 & c_{1} & c_{2} & d_{1} \\ & 1 & & \\ & & & 1 & \\ & & & & 1\end{array}\right)$, and $Z_{U T_{5}\left(\mathbf{F}_{q}\right)}(A, B, C)=\left(\begin{array}{ccccc}1 & & y_{1} & y_{2} & x_{2} \\ & 1 & z_{1} & z_{2} & w_{1} \\ & & 1 & & \\ & & & 1 & \\ & & & & 1\end{array}\right) .(A, B, C)$ is thus of type $R_{2}$, and there are $(q-1) q^{4}$ such branches.

When $c_{1}=0$, and $c_{2} \neq 0$. In Equation [7.19, we get $b_{1}^{\prime}=b_{1}$, and choose $x_{1}$ such that $b_{2}^{\prime}=0$. Hence on substituting $b_{2}$ with $b_{2}^{\prime}=0$ and equating Equation 7.19 , we get $x_{1}=0$. With this Equation 7.20 boils down to $d_{1}^{\prime}=d_{1}$, and Equation 7.21 boils down to $a_{2}^{\prime}=a_{2}$. $C$ is reduced to $\left(\begin{array}{ccccc}1 & & b_{1} & & a_{2} \\ & 1 & & c_{2} & d_{1} \\ & & 1 & & \\ & & & 1 & \\ & & & & 1\end{array}\right)$, and $Z(A, B, C)=\left(\begin{array}{ccccc}1 & & y_{1} & y_{2} & x_{2} \\ & 1 & z_{1} & z_{2} & w_{1} \\ & 1 & & \\ & & 1 & \\ & & & & 1\end{array}\right)$. $(A, B, C)$ is a branch of type $R_{1}$, and there are $q^{3}(q-1)$ such branches.

Case $a_{1} \neq 0$ : In this case, in Equation 7.19, we choose $z_{1}$ and $z_{2}$ such that $b_{1}^{\prime}=0$ and $b_{2}^{\prime}=0$ respectively. Thus, on replacing $b_{1}$ by $b_{1}^{\prime}=0$, and $b_{2}$ by $b_{2}^{\prime}=0$ in Equation 7.19 , and equating, we get $z_{1}=\frac{c_{1}}{a_{1}} x_{1}$ and $z_{2}=\frac{c_{2}}{a_{1}} x_{1}$. Putting these in Equation 7.20 leads us to $d_{1}+\left(\lambda \frac{c_{1}}{a_{1}} x_{1}+\frac{c_{2}}{a_{1}} x_{1}\right) a_{1}=d_{2}^{\prime}+\left(\lambda c_{1}+c_{2}\right) x_{1}$. Thus $d_{1}^{\prime}=d_{1}$.

With all this, Equation 7.21 boils down to $a_{2}+x_{1} d_{1}+\left(\lambda y_{1}+y_{2}\right) a_{1}=a_{2}^{\prime}+w_{1} a_{1}$. Choose $w_{1}$ so that $a_{2}^{\prime}=0$. Hence $C$ is reduced to $\left(\begin{array}{ccccc}1 & a_{1} & & & \\ & 1 & c_{1} & c_{2} & d_{1} \\ & 1 & & \lambda a_{1} \\ & & 1 & a_{1} \\ & & & 1\end{array}\right)$, and

$$
Z_{U T_{5}\left(\mathbf{F}_{q}\right)}(A, B)=\left(\begin{array}{ccccc}
1 & x_{1} & y_{1} & y_{2} & x_{2} \\
& 1 & \frac{c_{1}}{a_{1}} x_{1} & \frac{c_{2}}{a_{1}} x_{1} & \lambda y_{1}+y_{2}+\frac{d_{1}}{a_{1}} x_{1} \\
& 1 & & \lambda x_{1} \\
& & 1 & x_{1} \\
& & & & 1
\end{array}\right)
$$


Easy to check that the above centralizer subgroup is a commutative one, of size $q^{4}$. Thus $(A, B, C)$ is of type $R_{3}$, and there are $(q-1) q^{3}$ such branches.

Adding up all the branches of type $R_{1}$ gives us $q^{2}(q-1)+q^{3}(q-1)+q^{4}(q-1)=q^{5}-q^{2}$ branches of type $R_{1}$.

Proposition 7.18. The new type $U N T_{3}$ has $q^{3}$ branches of type $U N T_{3}, q^{4}-q^{2}$ branches of type $R_{2}$, and $q^{4}-q^{3}$ branches of type $R_{3}$.

Proof. A commuting pair $(A, B)$ of matrices in $U T_{5}\left(\mathbf{F}_{q}\right)$ of type $U N T_{3}$ has as its centralizer: $\left\{\left(\begin{array}{ccccc}1 & x_{1} & y_{1} & y_{2} & x_{2} \\ & 1 & \lambda_{1} x_{1} & z_{2} & w_{1} \\ & & 1 & \lambda_{2} x_{1} & \frac{\lambda_{2}}{\lambda_{1}} y_{1} \\ & & & 1 & x_{1} \\ & & & & 1\end{array}\right)\right\}$.

Let $C=\left(\begin{array}{ccccc}1 & a_{1} & b_{1} & b_{2} & a_{2} \\ & 1 & \lambda_{1} a_{1} & c_{2} & d_{1} \\ & & 1 & \lambda_{2} a_{1} & \frac{\lambda_{2}}{\lambda_{1}} b_{1} \\ & & & 1 & a_{1} \\ & & & & 1\end{array}\right)$, and $C^{\prime}=\left(\begin{array}{ccccc}1 & a_{1}^{\prime} & b_{1}^{\prime} & b_{2}^{\prime} & a_{2}^{\prime} \\ & 1 & \lambda_{1} a_{1}^{\prime} & c_{2}^{\prime} & d_{1}^{\prime} \\ & 1 & \lambda_{2} a_{1}^{\prime} & \frac{\lambda_{2}}{\lambda_{1}} b_{1}^{\prime} \\ & & 1 & a_{1}^{\prime} \\ & & & 1\end{array}\right)=$ $X C X^{-1}$, where $X=\left(\begin{array}{ccccc}1 & x_{1} & y_{1} & y_{2} & x_{2} \\ & 1 & \lambda_{1} x_{1} & z_{2} & w_{1} \\ & & 1 & \lambda_{2} x_{1} & \frac{\lambda_{2}}{\lambda_{1}} y_{1} \\ & & & 1 & x_{1} \\ & & & & 1\end{array}\right)$. From $X C=C^{\prime} X$, we get $a_{1}^{\prime}=a_{1}$, $b_{1}^{\prime}=b_{1}, c_{2}^{\prime}=c_{2}$, and the following equations:

$$
\begin{aligned}
b_{2}+x_{1} c_{2}+\lambda_{2} y_{1} a_{1} & =b_{2}^{\prime}+z_{2} a_{1}+\lambda_{2} x_{1} b_{1} \\
d_{1}+\lambda_{2} x_{1} b_{1}+z_{2} a_{1} & =d_{1}^{\prime}+\lambda_{2} y_{1} a_{1}+x_{1} c_{2} \\
a_{2}+x_{1} d_{1}+y_{2} a_{1} & =a_{2}^{\prime}+w_{1} a_{1}+x_{1} b_{2}^{\prime} .
\end{aligned}
$$

Case $a_{1}=0$ : Equation 7.22 becomes $b_{1}+x_{1} c_{2}=b_{1}^{\prime}+x_{1} \lambda_{2} b_{1}$. When $c_{2}=\lambda_{2} b_{1}$, then $b_{2}^{\prime}=$ $b_{2}$, and similarly in Equation 7.23, $d_{1}^{\prime}=d_{1}$. Here, if $b_{2}=d_{1}$, we get from Equation 7.24, $a_{2}^{\prime}=a_{2}$. Hence $C$ is reduced to $\left(\begin{array}{ccccc}1 & & b_{1} & b_{2} & a_{2} \\ & 1 & & \lambda_{2} b_{1} & b_{2} \\ & 1 & & \frac{\lambda_{2}}{\lambda_{1}} b_{1} \\ & & 1 & \\ & & & & 1\end{array}\right)$, and $Z_{U T_{5}\left(\mathbf{F}_{q}\right)}(A, B, C)=$ $Z_{U T_{5}\left(\mathbf{F}_{q}\right)}(A, B) .(A, B, C)$ is a branch of type $U N T_{3}$, and there are $q^{3}$ such branches. 
When $b_{2} \neq d_{1}$, choose $x_{1}$ such that $a_{2}^{\prime}=0 . C$ is reduced to $\left(\begin{array}{ccccc}1 & & b_{1} & b_{2} & \\ & 1 & & \lambda_{2} b_{1} & d_{1} \\ & & 1 & & \frac{\lambda_{2}}{\lambda_{1}} b_{1} \\ & & 1 & \\ & & & & 1\end{array}\right)$, and $Z_{U T_{5}\left(\mathbf{F}_{q}\right)}(A, B, C)=\left\{\left(\begin{array}{ccccc}1 & & y_{1} & y_{2} & x_{2} \\ & 1 & & z_{2} & w_{1} \\ & 1 & & \frac{\lambda_{2}}{\lambda_{1}} y_{1} \\ & & 1 & \\ & & & & 1\end{array}\right)\right\} \cdot(A, B, C)$ is thus of type $R_{2}$, and there are $q^{2}(q-1)$ such branches.

When $c_{2} \neq \lambda_{2} b_{1}$. In this case, in equation 7.22 itself, we choose $x_{1}$ such that $b_{2}^{\prime}=0$. And on substituting $b_{2}$ with 0 in this equation and equating, we get $x_{1}=0$. Thus, Equation 7.23 becomes $d_{1}^{\prime}=d_{1}$, and from Equation 7.24, we get $a_{2}^{\prime}=a_{2}$. Thus $C$ is reduced to $\left(\begin{array}{ccccc}1 & & b_{1} & & a_{2} \\ & 1 & & c_{2} & d_{1} \\ & & 1 & & \frac{\lambda_{2}}{\lambda_{1}} b_{1} \\ & & 1 & \\ & & & & 1\end{array}\right)$, and $Z_{U T_{5}\left(\mathbf{F}_{q}\right)}(A, B, C)=\left\{\left(\begin{array}{ccccc}1 & & y_{1} & y_{2} & x_{2} \\ & 1 & & z_{2} & w_{1} \\ & 1 & & \frac{\lambda_{2}}{\lambda_{1}} y_{1} \\ & & 1 & \\ & & & \\ & & & & \\ & & & & \\ & & & & \end{array}\right)\right\}$. This too is a branch of type $R_{2}$, and there are $q^{3}(q-1)$.

Case $a_{1} \neq 0$ : In Equation 7.22 choose $z_{2}$ such that $b_{2}^{\prime}=0$. Thus, substituting $b_{2}$ with $b_{2}^{\prime}=0$ in this equation, leads us to $z_{2}=\lambda_{2} y_{1}+\frac{\left(c_{2}-\lambda_{2} b_{1}\right)}{a_{1}} x_{1}$. With these Equation 7.23 becomes $d_{1}^{\prime}=d_{1}$. Thus Equation 7.24 becomes $a_{2}+x_{1} d_{1}+y_{2} a_{1}=a_{2}^{\prime}+w_{1} a_{1}$. Choose $w_{1}$ such that $a_{2}^{\prime}=0$. Thus $C$ is reduced to $\left(\begin{array}{ccccc}1 & a_{1} & b_{1} & & \\ & 1 & \lambda_{1} a_{1} & c_{2} & d_{1} \\ & 1 & \lambda_{2} a_{1} & \frac{\lambda_{2}}{\lambda_{1}} b_{1} \\ & & 1 & a_{1} \\ & & & 1\end{array}\right)$, and

$$
Z_{U T_{5}\left(\mathbf{F}_{q}\right)}(A, B, C)=\left\{\left(\begin{array}{ccccc}
1 & x_{1} & y_{1} & y_{2} & x_{2} \\
& 1 & \lambda_{1} x_{1} & \lambda_{2} y_{1}+\frac{\left(c_{2}-\lambda_{2} b_{1}\right)}{a_{1}} x_{1} & y_{2}+\frac{d_{1}}{a_{1}} x_{1} \\
& 1 & \lambda_{2} x_{1} & \frac{\lambda_{2}}{\lambda_{1}} y_{1} \\
& & & 1 & x_{1} \\
& & & & 1
\end{array}\right)\right\} .
$$

By a routine check, one can see that this centralizer group is commutative. Thus we have a branch of type $R_{3}$, and there are $(q-1) q^{3}$ such branches.

Adding up the branches of type $R_{2}$, there is a total of $q^{2}(q-1)+q^{3}(q-1)=q^{4}-q^{2}$ branches of type $R_{2}$. 


\section{Commuting Probabilities}

The number of simultaneous conjugacy classes of commuting $k$-tuples in $U T_{n}\left(\mathbf{F}_{q}\right)$ is denoted by $c_{U T}(n, k, q)$ and the same for $G T_{n}\left(\mathbf{F}_{q}\right)$ is denoted by $c_{G T}(n, k, q)$. From Lemma 7.1 [SS], it follows that $c_{G T}(n, k, q)=1 \cdot B_{G T_{n}\left(\mathbf{F}_{q}\right)}^{k} \cdot e_{1}$ and $c_{U T}(n, k, q)=1 \cdot B_{U T_{n}\left(\mathbf{F}_{q}\right)}^{k} \cdot e_{1}$ where $\mathbf{1}=\left(\begin{array}{llll}1 & 1 & \cdots & 1\end{array}\right)$, and $\mathbf{e}_{1}={ }^{t}\left(\begin{array}{lllll}1 & 0 & 0 & \cdots & 0\end{array}\right)$. We note that all of the branching matrices computed in this paper for triangular and unitriangular groups have entries polynomial in $q$ with integer coefficients. Thus, $c_{U T}(n, k, q)$ for $n=3,4,5$ and $c_{G T}(n, k, q)$ for $n=2,3,4$ are polynomials in $q$ with integer coefficients.

From Theorem 1.1 in [SS], for $k \geq 2$, and any finite group $G$, the probability that a $k$-tuple commutes is $c p_{k}(G)=\frac{c_{G}(k-1)}{|G|^{k-1}}=\frac{1 B_{G}^{k-1} \cdot e_{1}}{|G|^{k-1}}$. Now, that we have determined the branching matrix for the groups $G T_{i}\left(\mathbf{F}_{q}\right)$ for $i=2,3,4$, and $U T_{j}\left(\mathbf{F}_{q}\right)$ for $j=3,4,5$, for each of the groups, we will mention the commuting probabilities for $k \leq 5$. This computation is done using Sage [SA].

For the triangular groups we have:

\begin{tabular}{c|c||c|c}
\hline$k$ & $c p_{k}\left(G T_{2}\left(\mathbf{F}_{q}\right)\right)$ & $k$ & $c p_{k}\left(G T_{2}\left(\mathbf{F}_{q}\right)\right)$ \\
\hline 2 & $\frac{1}{q-1}$ & 4 & $\frac{q^{2}-2 q+4}{q^{5}-3 q^{4}+3 q^{3}-q^{2}}$ \\
& & & \\
3 & $\frac{q^{2}-q+2}{q^{4}-2 q^{3}+q^{2}}$ & 5 & $\frac{q^{4}-3 q^{3}+7 q^{2}-3 q+2}{q^{8}-4 q^{7}+6 q^{6}-4 q^{5}+q^{4}}$ \\
\hline
\end{tabular}

\begin{tabular}{c|c||c|c}
\hline$k$ & $c p_{k}\left(G T_{3}\left(\mathbf{F}_{q}\right)\right)$ & $k$ & $c p_{k}\left(G T_{3}\left(\mathbf{F}_{q}\right)\right)$ \\
\hline 2 & $\frac{q^{2}+q-1}{q^{3}(q-1)^{2}}$ & 4 & $\frac{q^{5}-3 q^{4}+7 q^{3}-5 q^{2}+11 q+4}{q^{8}(q-1)^{6}}$ \\
& & & $q^{3}-q^{2}+q+5$ \\
$q^{5}(q-1)^{4}$ & 5 & $\frac{q^{7}-5 q^{6}+17 q^{5}-32 q^{4}+54 q^{3}-34 q^{2}+25 q+2}{q^{11}(q-1)^{8}}$ \\
\hline
\end{tabular}

\begin{tabular}{c|c}
\hline$k$ & $c p_{k}\left(G T_{4}\left(\mathbf{F}_{q}\right)\right)$ \\
\hline 2 & $\frac{q^{3}+3 q^{2}-2 q-1}{q^{10}(q-1)^{3}}$ \\
3 & $\frac{12 q^{5}-52 q^{4}+116 q^{3}-97 q^{2}+63 q-37}{q^{20}(q-1)^{6}}$ \\
4 & $\frac{6 q^{8}-16 q^{7}+3 q^{6}+195 q^{5}-593 q^{4}+1105 q^{3}-1129 q^{2}+912 q-477}{q^{30}(q-1)^{9}}$ \\
5 & $\frac{7 q^{11}-32 q^{10}+122 q^{9}-192 q^{8}+342 q^{7}-714 q^{6}+2038 q^{5}-3954 q^{4}+6136 q^{3}-6304 q^{2}+4596 q-2213}{q^{40}(q-1)^{12}}$ \\
\hline
\end{tabular}

In the case of unitriangular group we have: 


\begin{tabular}{c|c||c|c}
\hline$k$ & $c p_{k}\left(U T_{3}\left(\mathbf{F}_{q}\right)\right)$ & $k$ & $c p_{k}\left(U T_{3}\left(\mathbf{F}_{q}\right)\right)$ \\
\hline 2 & $\frac{q^{2}+q-1}{q^{3}}$ & 3 & $\frac{q^{3}+q^{2}-1}{q^{5}}$ \\
& & & \\
4 & $\frac{q^{4}+q^{3}-1}{q^{7}}$ & 5 & $\frac{q^{5}+q^{4}-1}{q^{9}}$. \\
\hline
\end{tabular}

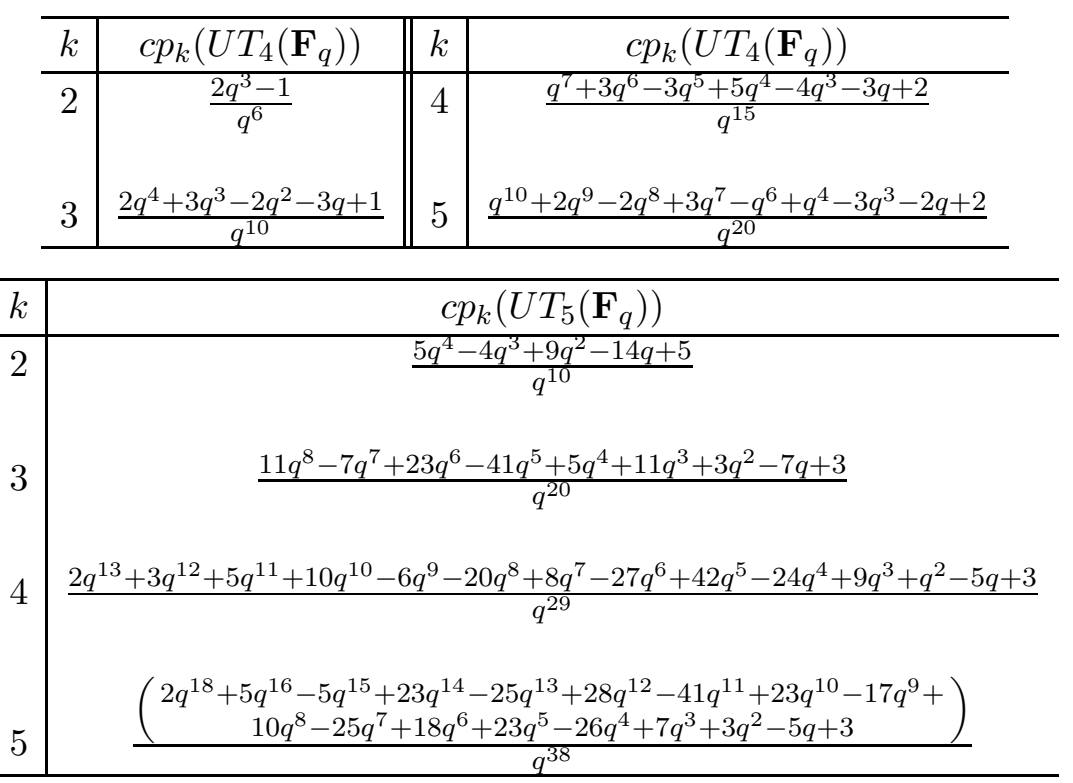

Appendix A. Conjugacy Classes of $G T_{4}\left(\mathbf{F}_{q}\right)$

The conjugacy classes for upper triangular group can be algorathmically computed following Belitskii's algorithm as described in [ $\mathrm{Ko}]$ and in the appendix of [Bh]. We list them here for the convenience of reader and also to set the notation for types.

\begin{tabular}{|c|c|c|c|}
\hline Class Representatives & $\begin{array}{c}\text { Number of } \\
\text { Classes }\end{array}$ & $\begin{array}{c}\text { Order of } \\
\text { Centralizer }\end{array}$ & $\begin{array}{c}\text { Name of } \\
\text { Type }\end{array}$ \\
\hline$a_{0} I_{4}, a_{0} \neq 0$ & $(q-1)$ & $(q-1)^{4} q^{6}$ & $C$ \\
\hline $\begin{array}{c}\left(\begin{array}{cccc}a & 1 & & \\
& a & & \\
& & a & \\
& & & \\
& & \end{array}\right),\left(\begin{array}{llll}a & & & \\
& a & & \\
& & a & 1 \\
& & & a\end{array}\right) \\
a \neq 0\end{array}$ & $2(q-1)$ & $(q-1)^{3} q^{4}$ & $A_{1}$ \\
\hline $\begin{array}{c}\left(\begin{array}{ccc}a & & \\
& a & 1 \\
& & a \\
& & a\end{array}\right) \\
a \neq 0\end{array}$ & $q-1$ & $(q-1)^{3} q^{4}$ & $A_{1}^{\prime}$ \\
\hline 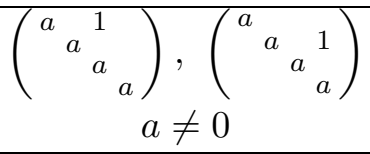 & $2(q-1)$ & $(q-1)^{3} q^{5}$ & $A_{2}$ \\
\hline 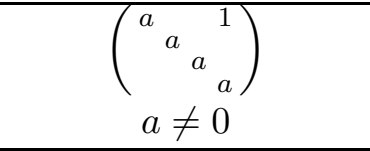 & $q-1$ & $(q-1)^{3} q^{6}$ & $A_{3}$ \\
\hline
\end{tabular}




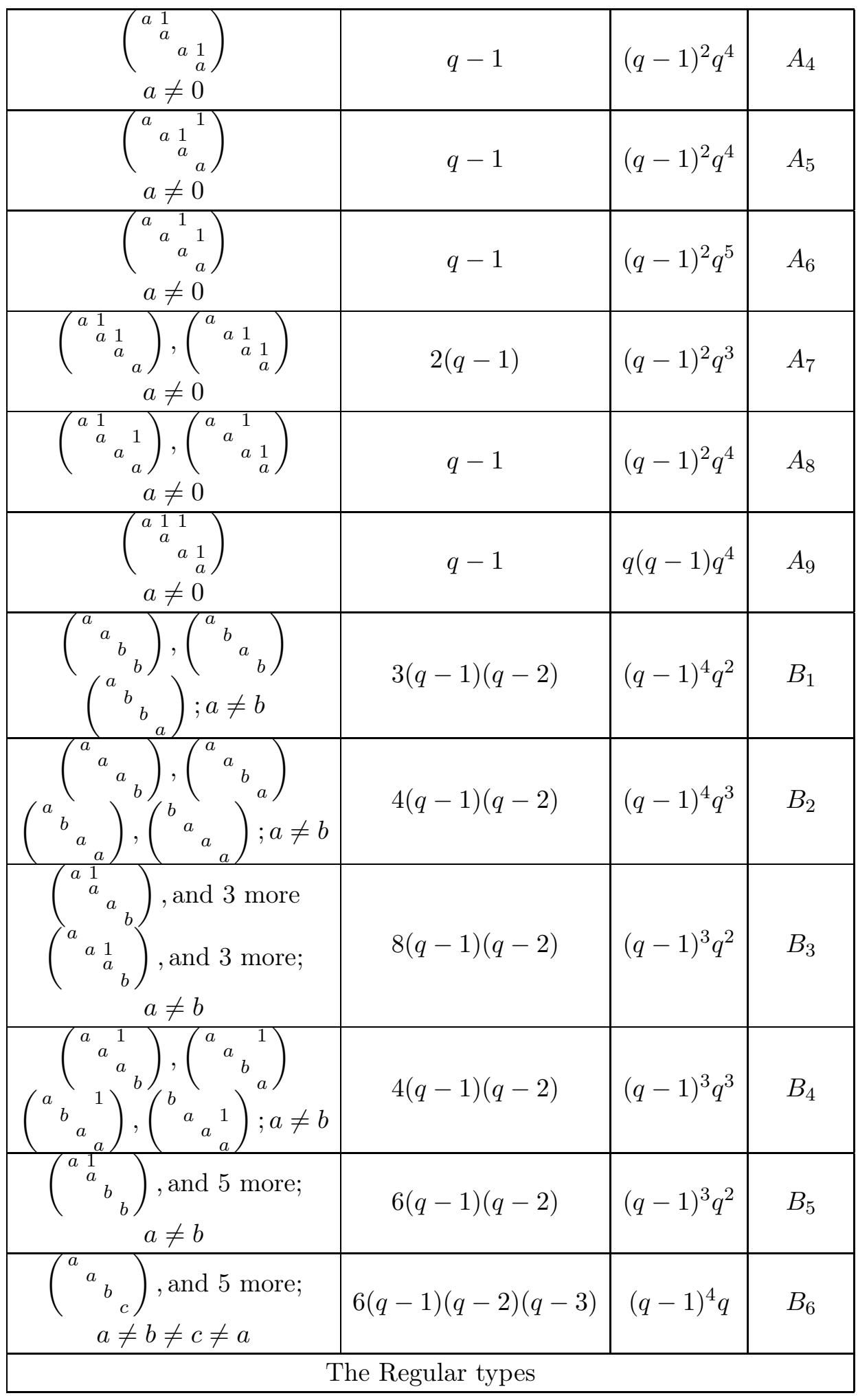




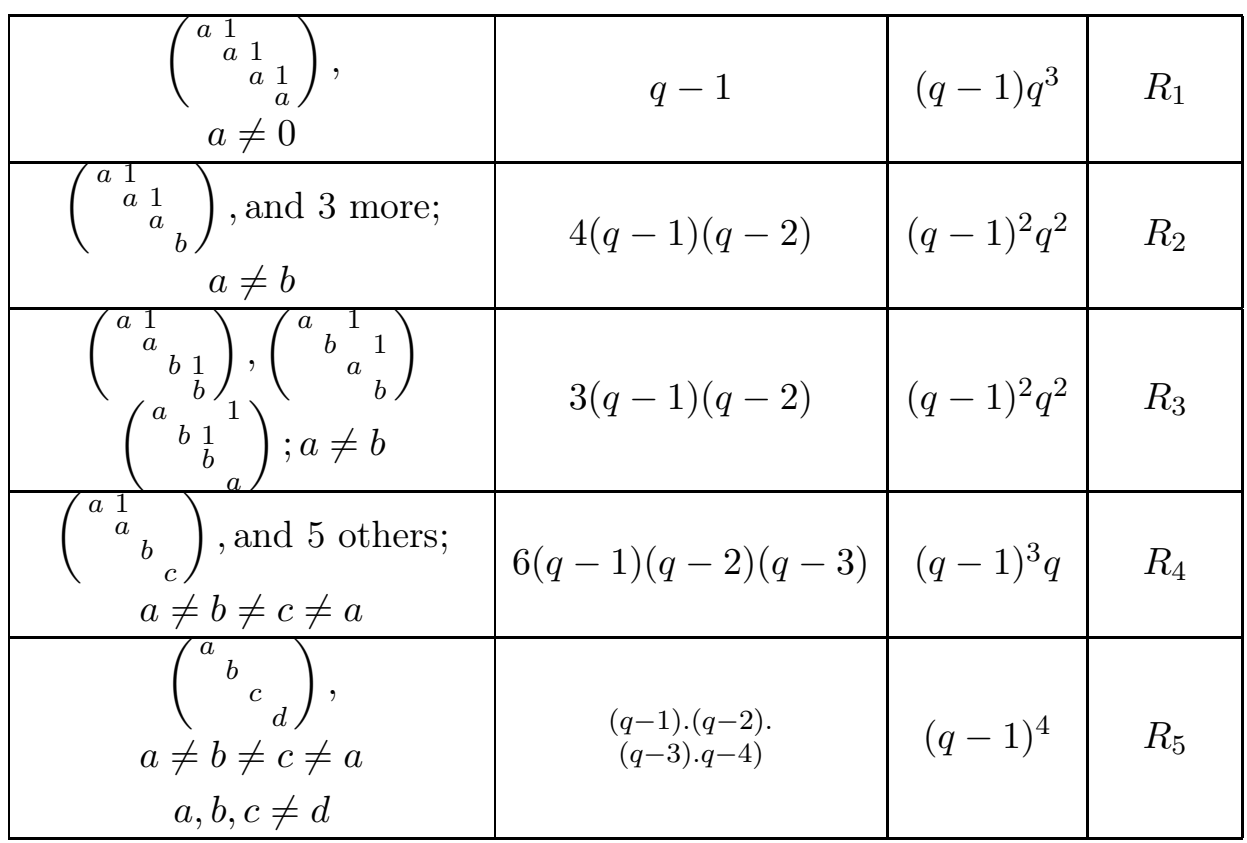

\section{Appendix B. Conjugacy Classes of $U T_{4}\left(\mathbf{F}_{q}\right)$ And $U T_{5}\left(\mathbf{F}_{q}\right)$}

Understanding conjugacy classes in unitriangular group is a challenging problem. We refer a reader to [VA1, VA2 for the reference. We list down the same for $\operatorname{UT}_{4}\left(\mathbf{F}_{q}\right)$ and $U T_{5}\left(\mathbf{F}_{q}\right)$, what we need for our purpose.

\begin{tabular}{|c|c|c|c|}
\hline Class Representatives & Number of Classes & $\begin{array}{c}\text { Centralizer size } \\
\text { in } U T_{4}\left(\mathbf{F}_{q}\right)\end{array}$ & Name of Type \\
\hline 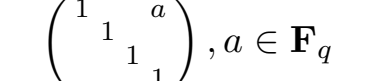 & $q$ & $q^{6}$ & $C$ \\
\hline 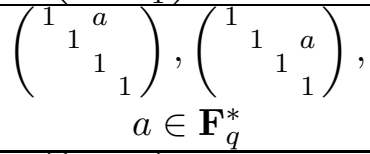 & $(q-1),(q-1)$ & $q^{5}$ & $A_{1}$ \\
\hline 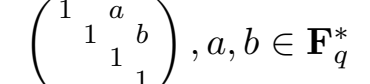 & $(q-1)^{2}$ & $q^{5}$ & $A_{2}$ \\
\hline 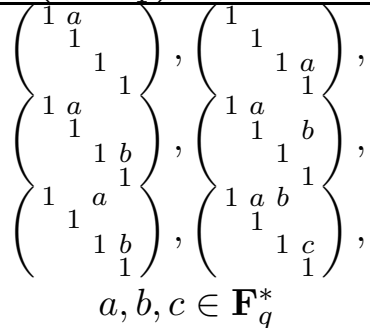 & $\begin{array}{l}(q-1),(q-1), \\
(q-1)^{2},(q-1)^{2}, \\
(q-1)^{2},(q-1)^{3}\end{array}$ & $q^{4}$ & $A_{3}$ \\
\hline
\end{tabular}




\begin{tabular}{|c|c|c|c|}
\hline $\begin{array}{c}\left(\begin{array}{cccc}1 & & & \\
& 1 & a \\
& & 1 & \\
& & & 1\end{array}\right),\left(\begin{array}{cccc}1 & & & b \\
& 1 & a & \\
& & 1 & \\
& & & 1\end{array}\right), \\
\\
\\
\end{array}$ & $(q-1),(q-1)^{2}$ & $q^{4}$ & $R_{1}$ \\
\hline $\begin{array}{l}\left(\begin{array}{cccc}1 & a & & \\
& 1 & b \\
& & 1 & \\
& & & \\
1 & a & & 1 \\
& 1 & b & \\
& & 1 & c \\
& & c\end{array}\right),\left(\begin{array}{cccc}1 & & & \\
& 1 & b & \\
& & 1 & a \\
& & 1\end{array}\right), a, b, c \in \mathbf{F}_{q}^{*}\end{array}$ & $\begin{array}{c}(q-1)^{2},(q-1)^{2} \\
(q-1)^{3}\end{array}$ & $q^{3}$ & $R_{2}$ \\
\hline
\end{tabular}

\begin{tabular}{|c|c|c|c|}
\hline Class Representatives & $\begin{array}{c}\text { Number of } \\
\text { Classes }\end{array}$ & $\begin{array}{c}\text { Order of } \\
\text { Centralizer in } U T_{5}\left(\mathbf{F}_{q}\right)\end{array}$ & $\begin{array}{l}\text { Name of } \\
\text { Type }\end{array}$ \\
\hline $\left.\begin{array}{lll}1 & & \\
& 1 & \\
& & 1\end{array}\right), a \in \mathbf{F}_{q}$ & $q$ & $q^{10}$ & $C$ \\
\hline 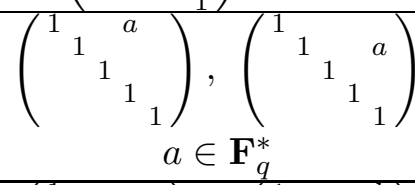 & $(q-1),(q-1)$ & $q^{9}$ & $A_{1}$ \\
\hline 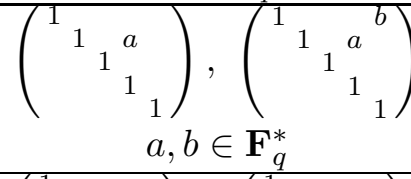 & $(q-1),(q-1)^{2}$ & $q^{8}$ & $A_{2}$ \\
\hline 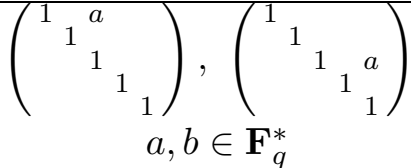 & $\begin{array}{l}(q-1),(q-1)^{2} \\
(q-1),(q-1)^{2}\end{array}$ & $q^{8}$ & $A_{3}$ \\
\hline 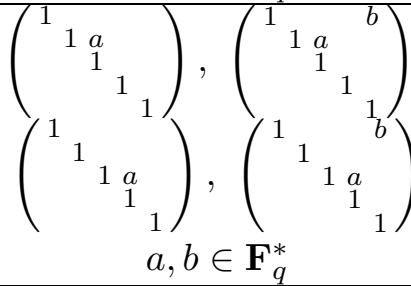 & $\begin{array}{l}(q-1),(q-1)^{2} \\
(q-1),(q-1)^{2}\end{array}$ & $q^{7}$ & $A_{4}$ \\
\hline 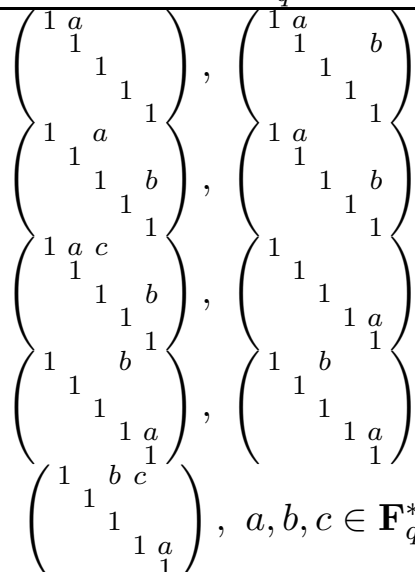 & $\begin{array}{c}(q-1),(q-1)^{2}, \\
(q-1)^{2},(q-1)^{2}, \\
(q-1)^{3},(q-1), \\
(q-1)^{2},(q-1)^{2}, \\
(q-1)^{3}\end{array}$ & $q^{7}$ & $A_{5}$ \\
\hline
\end{tabular}




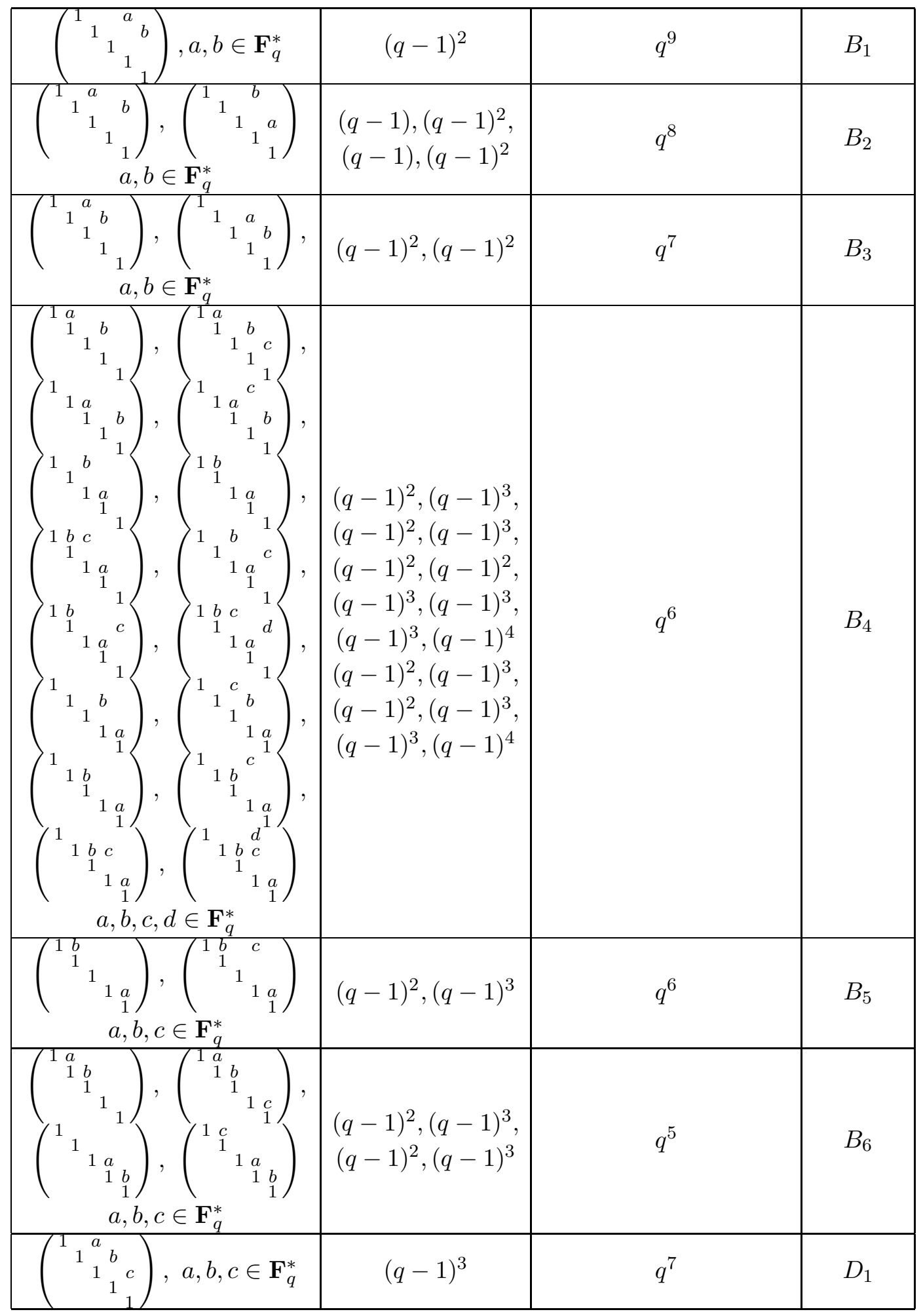




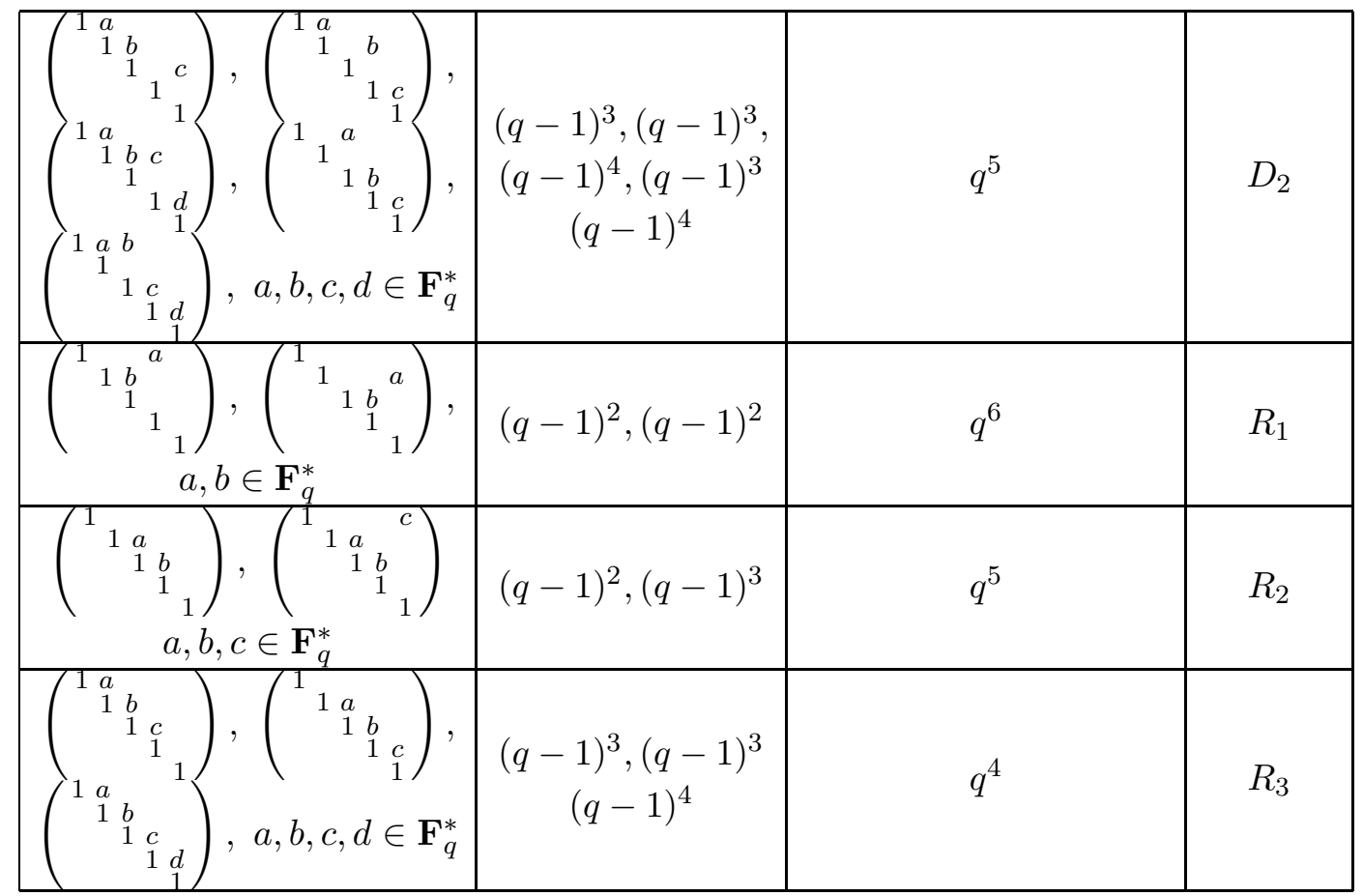

\section{REFERENCES}

[Bh] Bhunia, Sushil, "Conjugacy classes of centralizers in the group of upper triangular matrices", Journal of algebra and its applications, vol 19, no. 1 (2020).

[GR] Guralnick, Robert M.; Robinson, Geoffrey R., "On the commuting probability in finite groups", J. Algebra 300 (2006), no. 2, 509-528.

[Ko] Kobal, Damjan, "Belitskii's canonical form for $5 \times 5$ upper triangular matrices under upper triangular similarity", Linear Algebra Appl. 403 (2005), 178-182.

[KSS] Kaur, Dilpreet; Sharma, Uday Bhaskar; Singh, Anupam, "Branching Rules and commuting probabilities of triangular and unitriangular Matrices", Arxiv:????

[SA] SageMath, the Sage Mathematics Software System (Version 8.3), The Sage Developers, 2019, https://www.sagemath.org.

[Sh1] Sharma, Uday Bhaskar, "Simultaneous Similairty Classes of Commuting Matrices over a Finite Field", Linear Algebra and its Applications, 501 (2016) 48-97.

[SS] Sharma, Uday Bhaskar; Singh, Anupam, "Branching Rules for Unitary and Symplectic Matrices", Communications in Algebra (published online, March 2020)., DOI: 10.1080/00927872.2020.1726366

[SS2] Sharma, Uday Bhaskar; Singh, Anupam, "Commuting probability and simultaneous conjugacy classes of commuting tuples in a group", arXiv: 2002.01253.

[VA1] Vera López, Antonio; Arregi, Jesus Maria, "Conjugacy classes in Sylow p-subgroups of $G L(n, q)$ ", J. Algebra 152 (1992), no. 1, 1-19. 
[VA2] Vera-López, A.; Arregi, J. M., "Conjugacy classes in Sylow p-subgroups of GL $(n, q)$. II", Proc. Roy. Soc. Edinburgh Sect. A 119 (1991), no. 3-4, 343-346.

E-mail address: dilpreetmaths@gmail.com

Department of Mathematics, Indian Institute of Technology (IIT) Jodhpur, NH 65, Surpura Bypass Rd, Karwar, Rajasthan 342037

E-mail address: udaybsharmaster@gmail.com

Tata Institute of Fundamental Research, Dr. Homi Bhabha Road, Navy Nagar, Colaba, Mumbai 400005, INDiA

E-mail address: anupamk18@gmail.com

Indian Institute of Science Education and Research (IISER) Pune, Dr. Homi Bhabha Road Pashan, Pune 411008, India 